SELECTED TRACE-ELEMENT DATA FOR STREAMS

IN THE SOUTHERN YAMPA RIVER BASIN,

NORTHWESTERN COLORADO

By Wendy S. Maura

U.S. GEOLOGICAL SURVEY

Open-file Report 85-192

Prepared in cooperation with the

U.S. BUREAU OF LAND MANAGEMENT

Lakewood, Colorado

1985 


\section{UNITED STATES DEPARTMENT OF THE INTERIOR \\ DONALD PAUL HODEL, Secretary \\ GEOLOGICAL SURVEY \\ Dallas L. Peck, Director}

For additional information write to:

District Chief U.S. Geological Survey Box 25046, Mail Stop 415 Denver Federal Center Lakewood, CO 80225
Copies of this report may be purchased from:

Open-File Services Section Western Distribution Branch U.S. Geological Survey Box 25425, Federal Center Denver, CO 80225

Telephone: (303) 236-7476 
Abstract-1

Introduction-1

Description of study area-1

Description of data-......... 2

References-0.- 5

\section{ILLUSTRATION}

Figure 1. Map of showing location of surface-water gaging stations and selected surface-water sampling sites in the study area-----

Page 3

TABLES

Table 1. Water-quality study sites in the southern Yampa River basin---- 6

2. Surface-water gaging stations in the southern Yampa River basin where water-quality data are collected-_.-.

3. Water-quality data collected at study sites in the southern Yampa River basin:

09238000 Oak Creek near Oak Creek-10 8

09244100 Fish Creek near Milner- 10

09244300 Grassy Creek near Mount Harris-_._. 11

09250000 Milk Creek near Thornburgh-_... 13

401601107375400 Morapos Creek near Iles Grove-_........ 14

401601107395300 Stinking Gulch near Thornburgh-_. 16

401747107161600 Willow Creek near Dunckley-............ 20

401829107375600 Deer Creek near Hamilton-.

401857107243500 South Fork of Williams Fork at mouth

near Pagoda-101925107523500 24

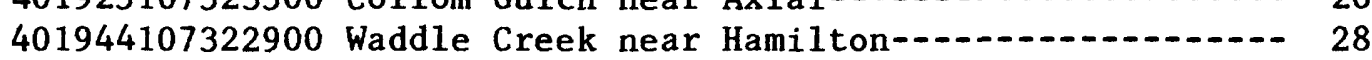

401948107445600 Milk Creek near Iles Grove-............ 30

402330107082000 Grassy Creek at Grassy Gap-_............ 32

$402530106585700 \mathrm{Fish}$ Creek at mouth near Milner-_-.-.-.-- 34

402605107181500 Dill Gulch near Hayden-_. 40

402720106591200 Trout Creek above Milner-_..._. 41

402829107193700 Smuin Gulch near Hayden-............... 43

402836106550100 Cow Creek near Steamboat Springs-.......-... 44

Table 4. Summary of selected water-quality data collected at surfacewater gaging stations in the southern Yampa River basin:

09243700 Middle Creek near Oak Creek-............. 45

09243800 Foide1 Creek near Oak Creek-...-...- 55

09243900 Foidel Creek at mouth, near Oak Creek--.---- 67

09244415 Sage Creek above Sage Creek Reservoir,

near Hayden-.... 78

09244460 Watering Trough Gulch near Hayden-....... 85

09244464 Hubberson Gulch near Hayden-... 93 
Table 4--Continued:

09244470 Stokes Gulch near Hayden-_._. 106

09250507 Wilson Creek above Taylor Creek near Axial--- 112

09250510 Taylor Creek at mouth near Axia1-......... 116

09250600 Wilson Creek near Axial_... 127

09250610 Jubb Creek near Axial _. 135

09250700 Morgan Gulch near Axia1_._. 145

Table 5. Statistical summary of water-quality data collected at selected surface-water gaging stations in the Yampa River basin:

09244410 Yampa River below diversion, near Hayden-- - 149

09247600 Yampa River below Elkhead Creek near Craig-- 150

09247600 Yampa River below Craig_................ 151

09249750 Williams Fork at mouth, near Hamilton-....-. 152

09250400 Good Spring Creek at Axial_._. 153

09251000 Yampa River near Maybe11_.................. 154

\section{CONVERSION FACTORS}

The inch-pound units used in this report may be converted to SI (International System) units by using the following conversion factors:

Multiply inch-pound units

acre-foot (acre-ft)

cubic foot per second $\left(\mathrm{ft}^{3} / \mathrm{s}\right)$

square mile $\left(\mathrm{mi}^{2}\right)$

ton, short, per acre-foot

(ton/acre-ft)

ton, short, per day (ton/d)
By

0.001233

0.2832

2.590

1.119

907.2
To obtain metric units

cubic hectometer

cubic meter per second square kilometer

kilogram per cubic hectometer

kilogram per day

Degree Celsius $\left({ }^{\circ} \mathrm{C}\right)$ may be converted to degree Fahrenheit $\left({ }^{\circ} \mathrm{F}\right)$ by using the following equation:

$$
{ }^{\circ} \mathrm{F}=9 / 5^{\circ} \mathrm{C}+32
$$

The following terms and abbreviations also are used in this report:

microgram per liter $(\mu \mathrm{g} / \mathrm{L})$

microsiemens per centimeter $(\mu \mathrm{S} / \mathrm{cm})$

milligram per liter $(\mathrm{mg} / \mathrm{L})$ 


\title{
SELECTED TRACE-ELEMENT DATA FOR STREAMS IN THE SOUTHERN YAMPA RIVER BASIN, NORTHWESTERN COLORADO
}

\author{
By Wendy S. Maura
}

\begin{abstract}
Increases in coal mining in northwestern Colorado have increased concerns about the impact of mining on the chemical quality of surface water. An area of particular concern is in the southern part of the Yampa River basin. To identify the changes produced by mining in this area, it is necessary to determine the present water chemistry resulting from the geology, climate, and land use. Because few data were available, a program for the synoptic collection of water-quality data was developed, in which sampling sites were selected to determine the surface-water chemistry.

This study was begun in April 1982. Water-quality samples were collected from sites on continuously flowing streams where a large concentration of suspended material was present. Each selected site, from the Oak Creek drainage near Steamboat Springs on the east, to the surface-water gaging station on the Yampa River near Maybell on the west, was sampled repetitively as changes occurred in discharge and specific conductance. Water-quality data from surface-water gaging stations and other selected surface-water sampling sites in the study area for water years 1976 to 1982 are included in the report.
\end{abstract}

\section{INTRODUCTION}

Increasing demands for energy have resulted in a significant increase of coal production in Colorado, particularly in the Yampa River basin of northwestern Colorado. This development has increased concerns about the impacts of mining on the quality of surface water. To identify the possible changes in water quality produced by mining in this area, it is first necessary to determine the present water chemistry resulting from the geology, climate, and land use. A synoptic water-quality program was developed to assess the water chemistry of the Yampa River basin.

This study began in April 1982, in cooperation with the U.S. Bureau of Land Management. It was a continuation of a 1981 study (Maura, 1982) in which water-quality samples were collected to determine concentrations of the major dissolved constituents present in streams of this study area. The objective of this report is to make available the trace-element data collected for selected streams in this study area.

\section{DESCRIPTION OF STUDY AREA}

Data presented in this publication were collected from some of the sites sampled in the 1981 study (Maura, 1982). In that study, 26 sites were sampled 
in the synoptic data-collection program. The criterion for selecting a stream for sampling was that the stream be flowing in April 1981.

In this study, 18 sites were chosen from the original 26 to collect trace-element data. The reduced number of sites results from an additional criterion that a site must have a large concentration of suspended material. In addition to these 18 sites sampled during the study period, 18 established surface-water gaging stations were selected that had similar trace-element data available. Locations of these established surface-water gaging stations and additional surface-water sampling sites are shown in figure 1.

The area of data collection was the southern part of the Yampa River basin between the confluence with Oak Creek upstream from Steamboat Springs, downstream to the surface-water gaging station, 09251000 Yampa River near Maybell. This downstream station was chosen as the western limit of the study area because it is included in the National Stream Quality Accounting Network (NASQAN), and it is a station for which a large number of water-quality analyses are available.

\section{DESCRIPTION OF DATA}

At each study site, water samples were collected as discharge decreased through the season. As flow decreased, suspended-sediment concentration generally decreased. When the sediment concentration was less than $100 \mathrm{mg} / \mathrm{L}$, data collection was discontinued at the site.

The instantaneous discharge of the stream was measured at the time of each sample collection, and the onsite measurements of water temperature, $\mathrm{pH}$, and specific conductance were recorded. Analyses of the water-quality samples were performed at the Denver Central Laboratory of the U.S. Geological Survey, Arvada, Colo.

The individual trace elements and suspended sediment properties analyzed, with units of measurement, are listed below:

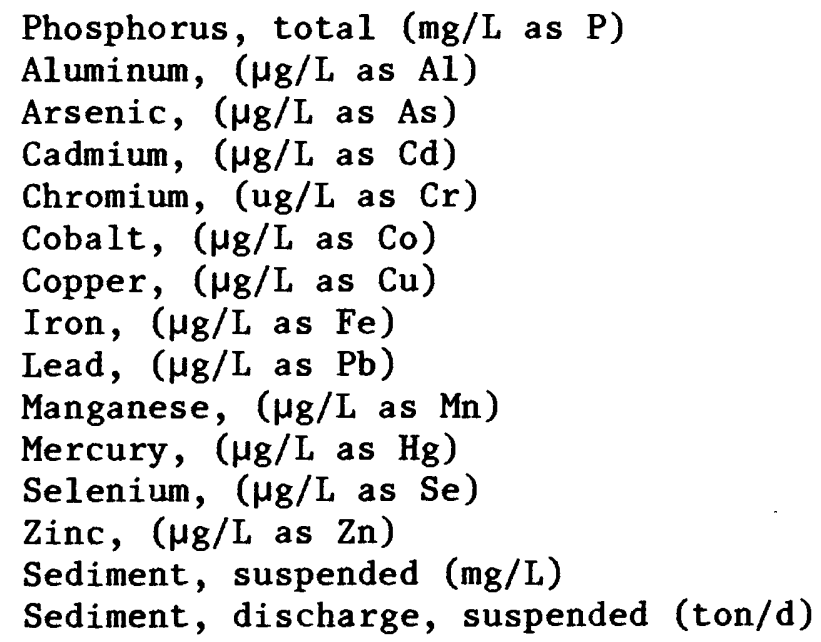



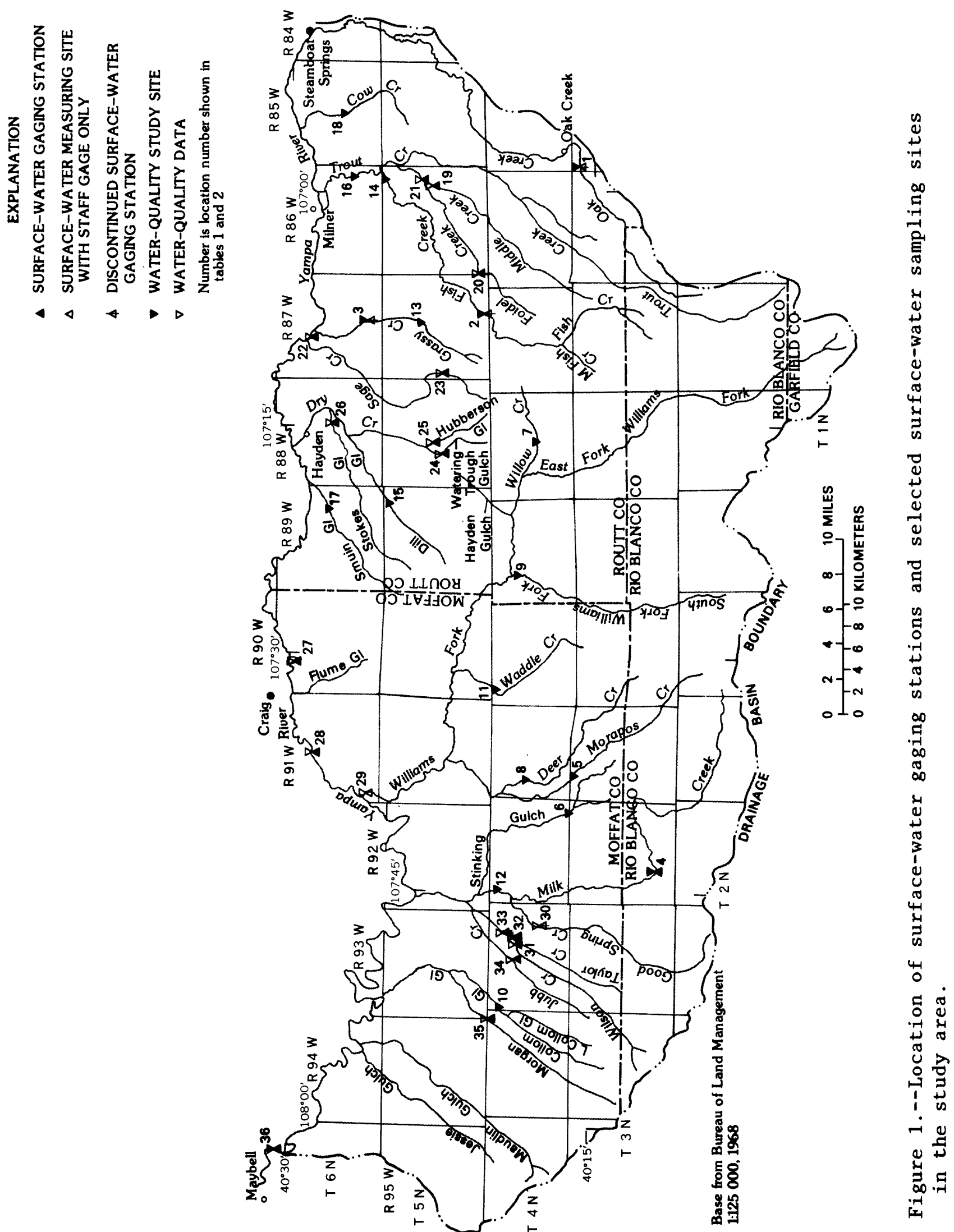
These trace elements (except phosphorus) were analyzed for total, dissolved, and suspended concentrations.

The location of each water-quality sampling site is shown in figure 1 , and a description of each site is given in table 1. Surface-water gaging stations in the study area where both sediment and water-quality data-were already available are described in table 2. There are additional surfacewater gaging stations in the study area that are not listed in table 2 or shown in figure 1 because they record only streamflow. Many stations listed in table 2 are on the main stem of the Yampa River or its principal tributary, the Williams Fork. These stations are part of the long-term gaging network of the U.S. Geological Survey. Many of the surface-water gaging stations on the smaller tributaries in the study area were operated in cooperation with U.S. Bureau of Land Management as part of the U.S. Geological Survey Coal Hydrology Program.

Data collected at the study sites are listed in table 3 . Water-quality constituents and properties determined from samples collected at U.S. Geological Survey surface-water gaging stations are listed in table 4. Data are listed only for the same constituents collected at the study sites. Waterquality data collected prior to water year 1976, and for additional waterqualty constituents, are available for some of these stations and are published annually in a series of the U.S. Geological Survey water-data reports entitled "Water Resources Data--Colorado."

A statistical summary of the constituents reported in tables 3 and 4 is given in table 5. This table contains a summary for six surface-water gaging stations located on the Yampa River, the Williams Fork, and Good Springs Creek. Many samples were collected at these six stations; therefore, only a summary of the data is given, which includes the number of samples, the mean, the standard deviation, and the range of each constituent for the period of record. The actual data are stored in WATSTORE, the U.S. Geological Survey's national water data storage and retrieval system.

\section{ABBREVIATIONS AND SYMBOLS}

The following abbreviations and symbols are used in tables 1 through 5 .

${ }^{\circ} \mathrm{C}$ is degrees Celsius;

$\mathrm{E}$ is estimated;

$\mathrm{ft}^{3} / \mathrm{s}$ is cubic feet per second;

$\mu \mathrm{S} / \mathrm{cm}$ is microsiemens per centimeter;

$\mathrm{mg} / \mathrm{L}$ is milligrams per liter;

$\mathrm{N}$ is number of water-quality samples;

ND is not determined;

$\mathrm{mi}^{2}$ is square miles; and

ton/d is tons per day. 


\section{REFERENCES}

Maura, W.S., 1982, Water-quality data for streams in the southern Yampa River basin, Northwestern Colorado: U.S. Geological Survey Open-File Report 82-1017, 112 p.

U.S. Geological Survey, 1976, Water resources data for Colorado, water year 1976, Volume 2. Colorado River Basin: U.S. Geological Survey Water-Data Report C0-76-2, available only from National Technical Information Service, Springfield, Va., as PB-278780.

U.S. Geological Survey, issued annually, Water resources data for Colorado (for water years 1977-1981), Volume 3. Dolores River basin, Green River basin, and San Juan River basin: U.S. Geological Survey WaterData Reports C0-77-3, CO-78-3, C0-79-3, CO-80-3, C0-81-3, CO-82-3, available only from National Technical Information Service, Springfield, Va., as PB-293522, PB80-119969, PB80-217979, PB82-202045, and PB83-124446. 


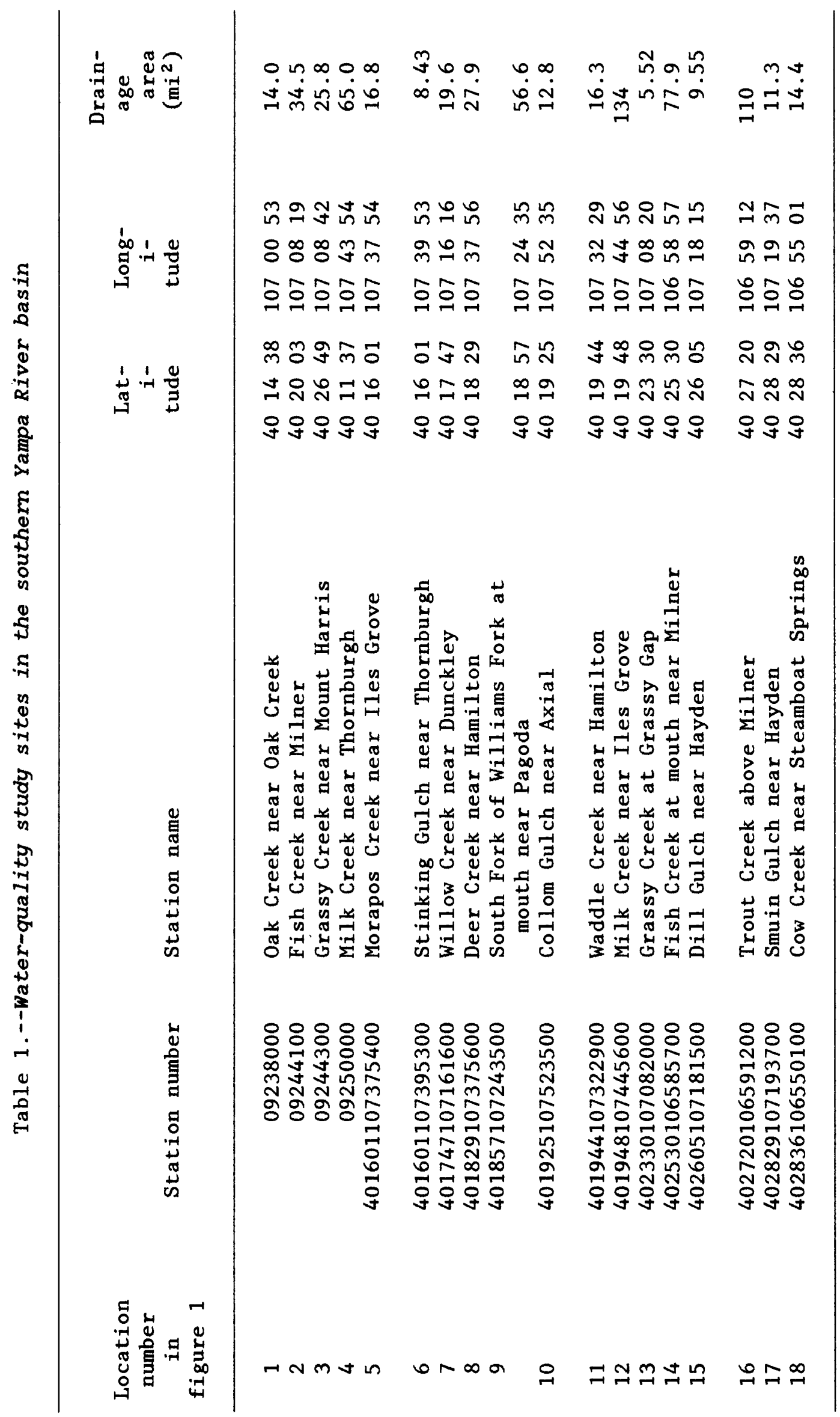




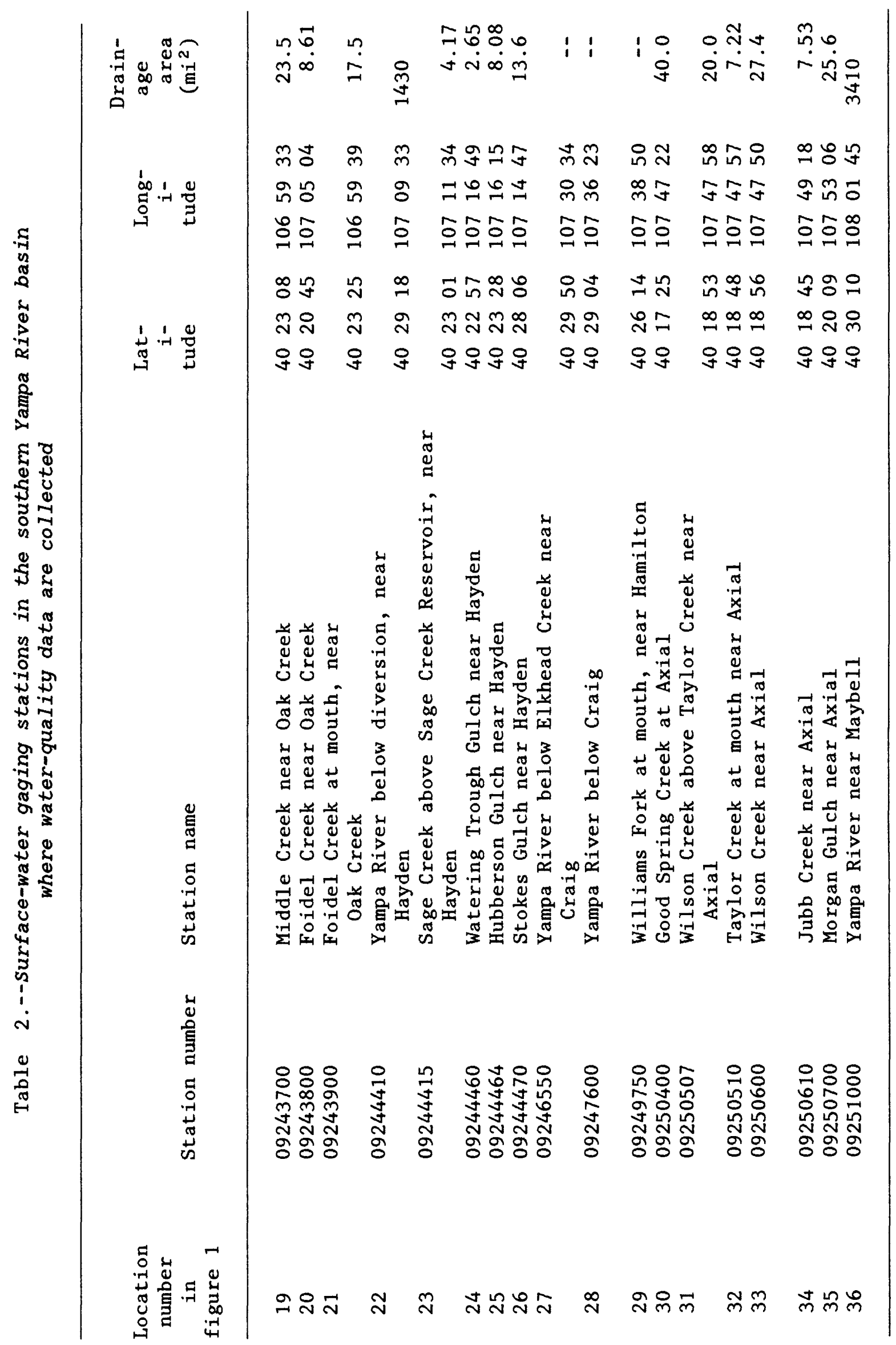




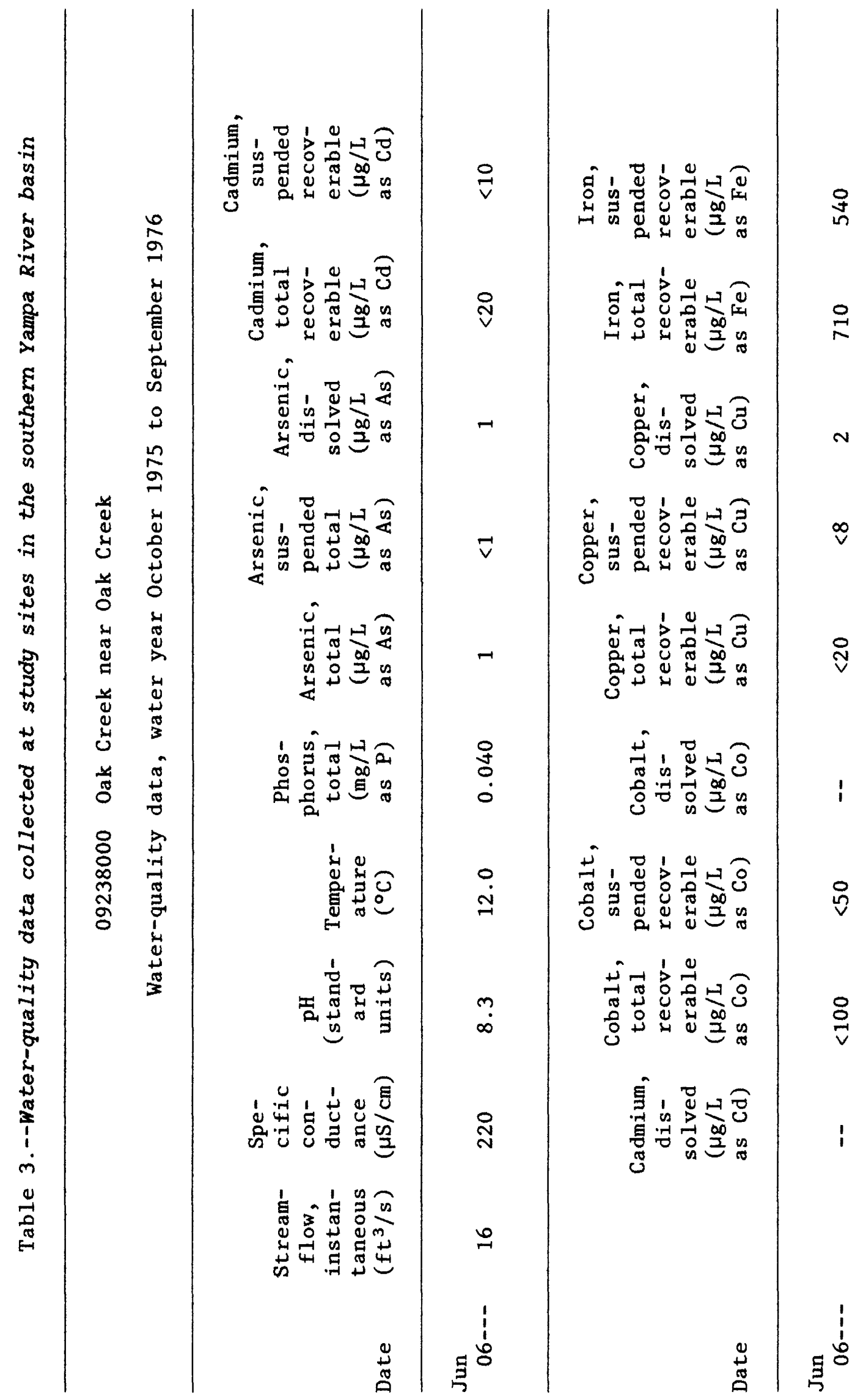




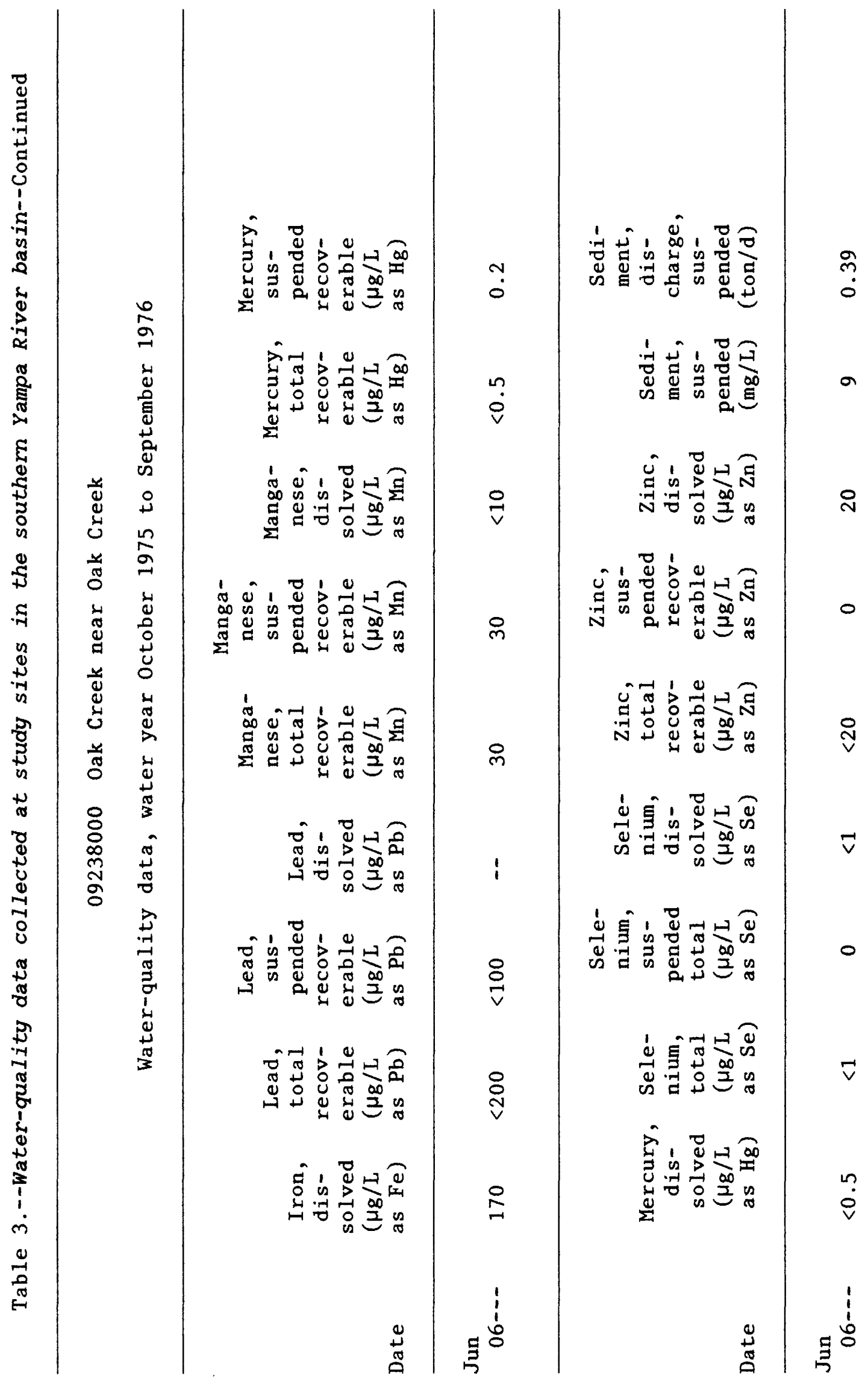




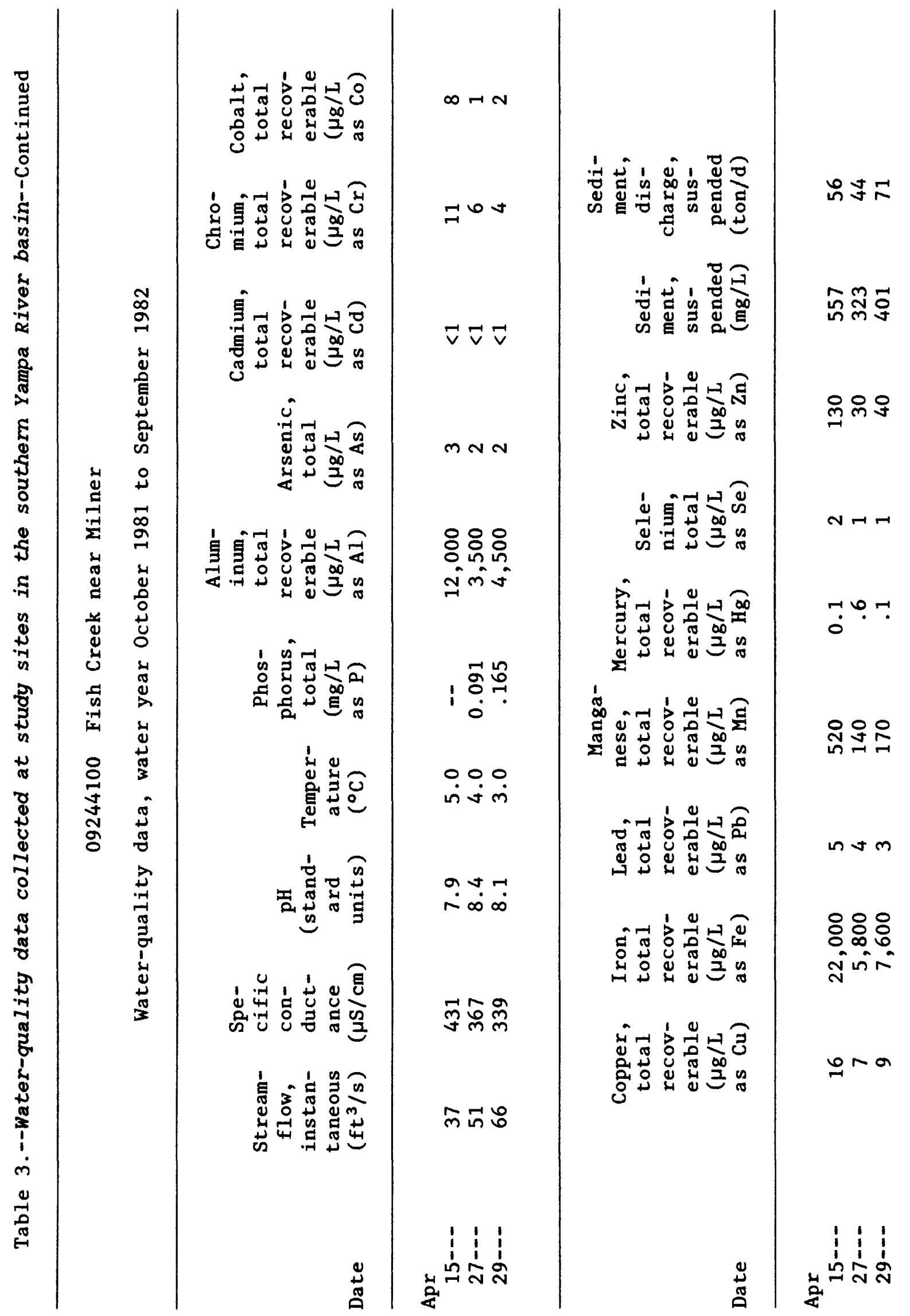




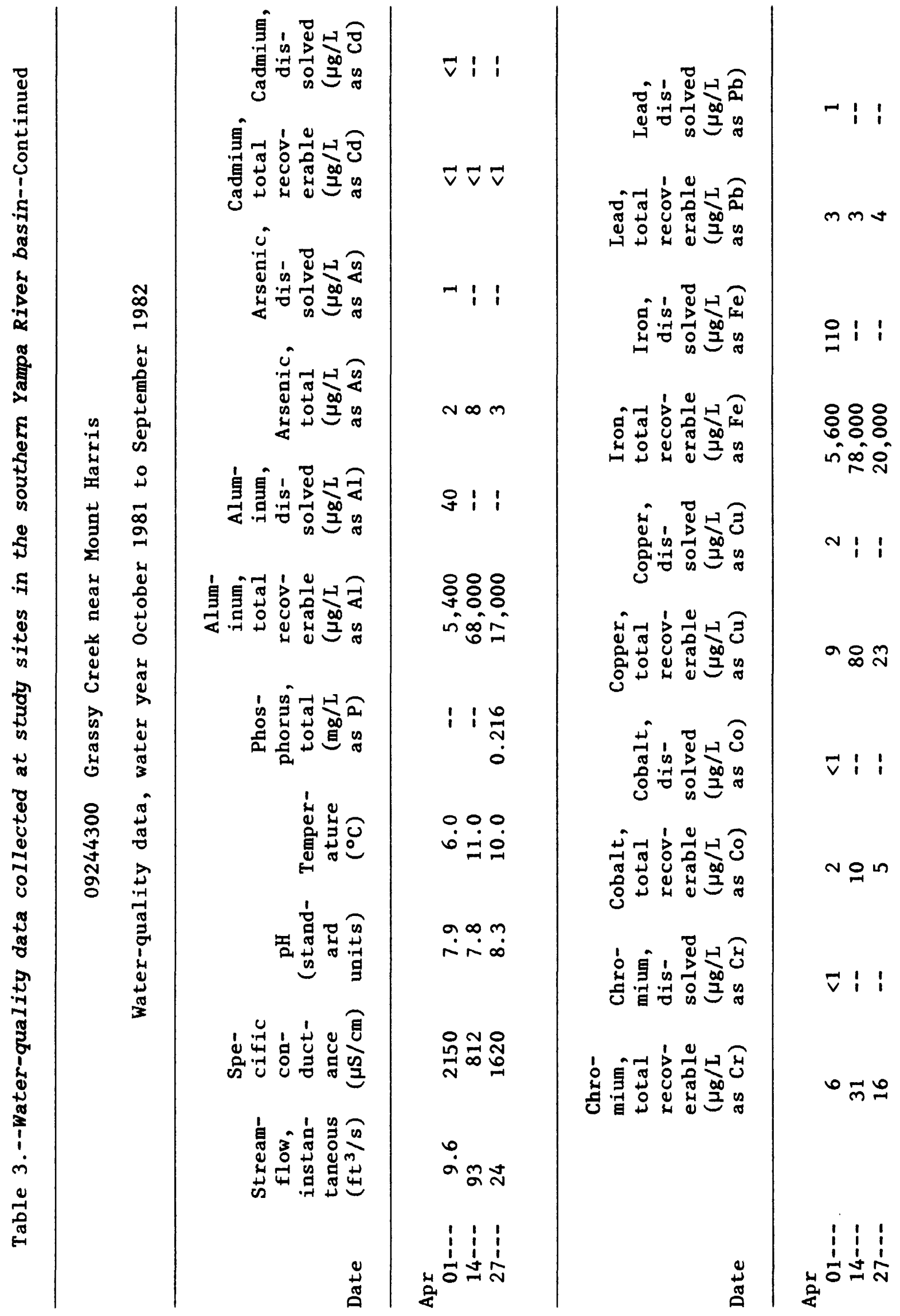




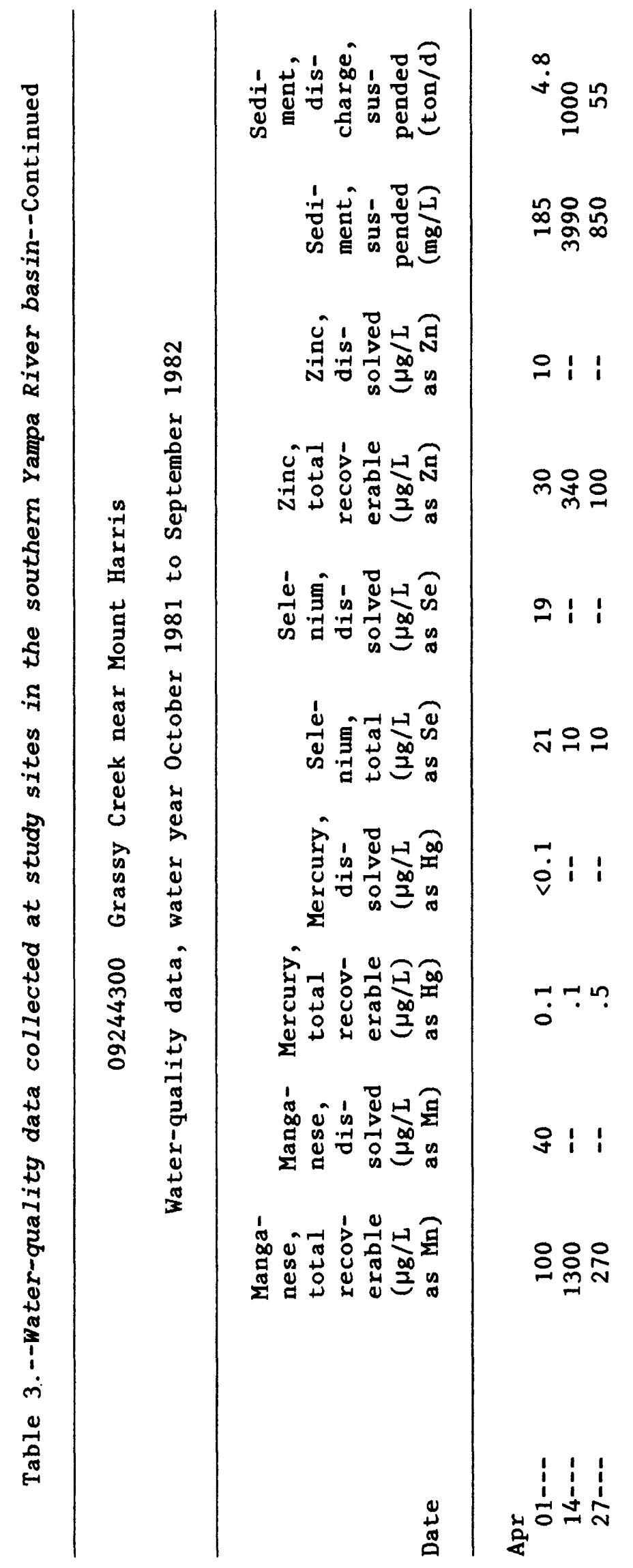




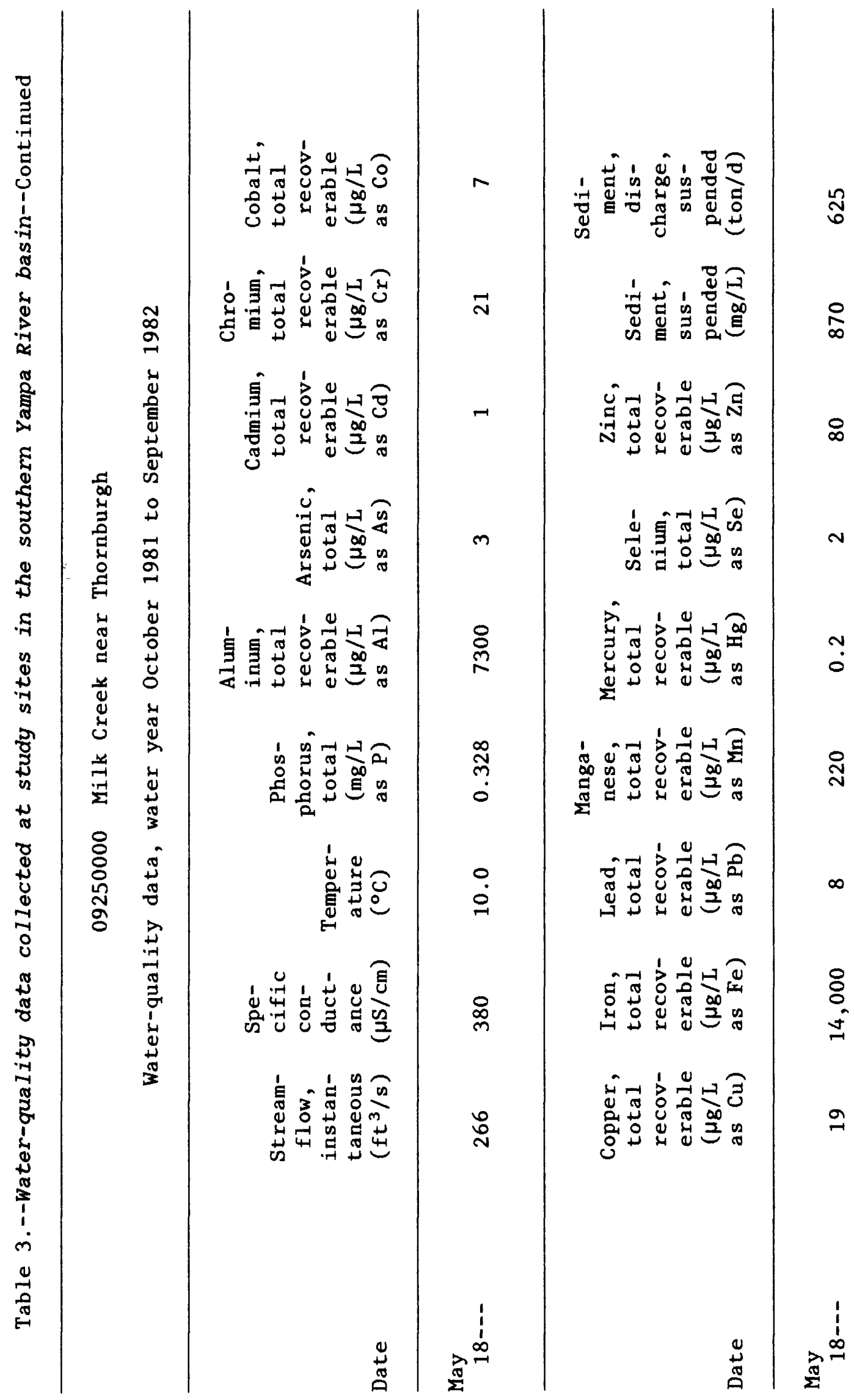




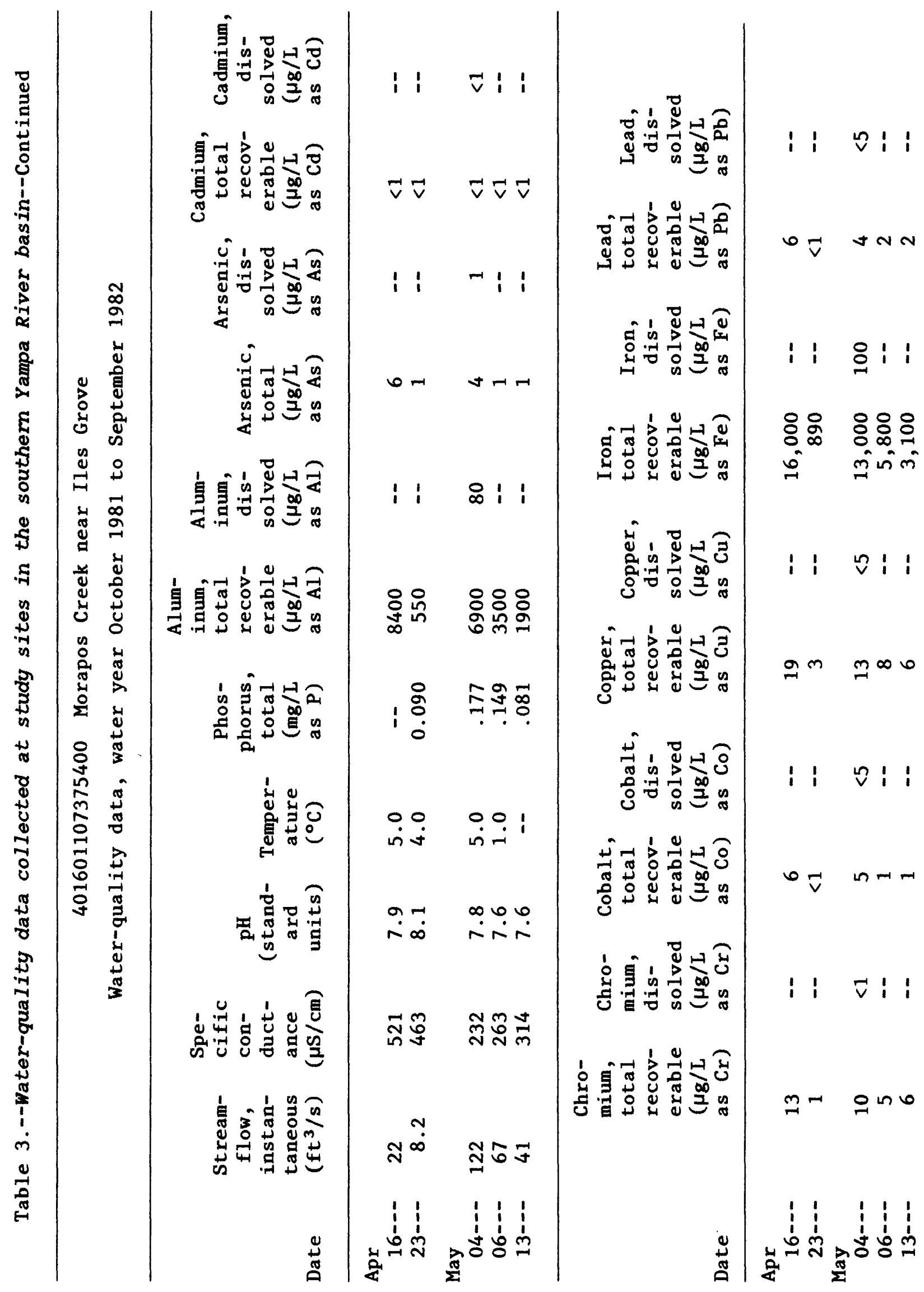




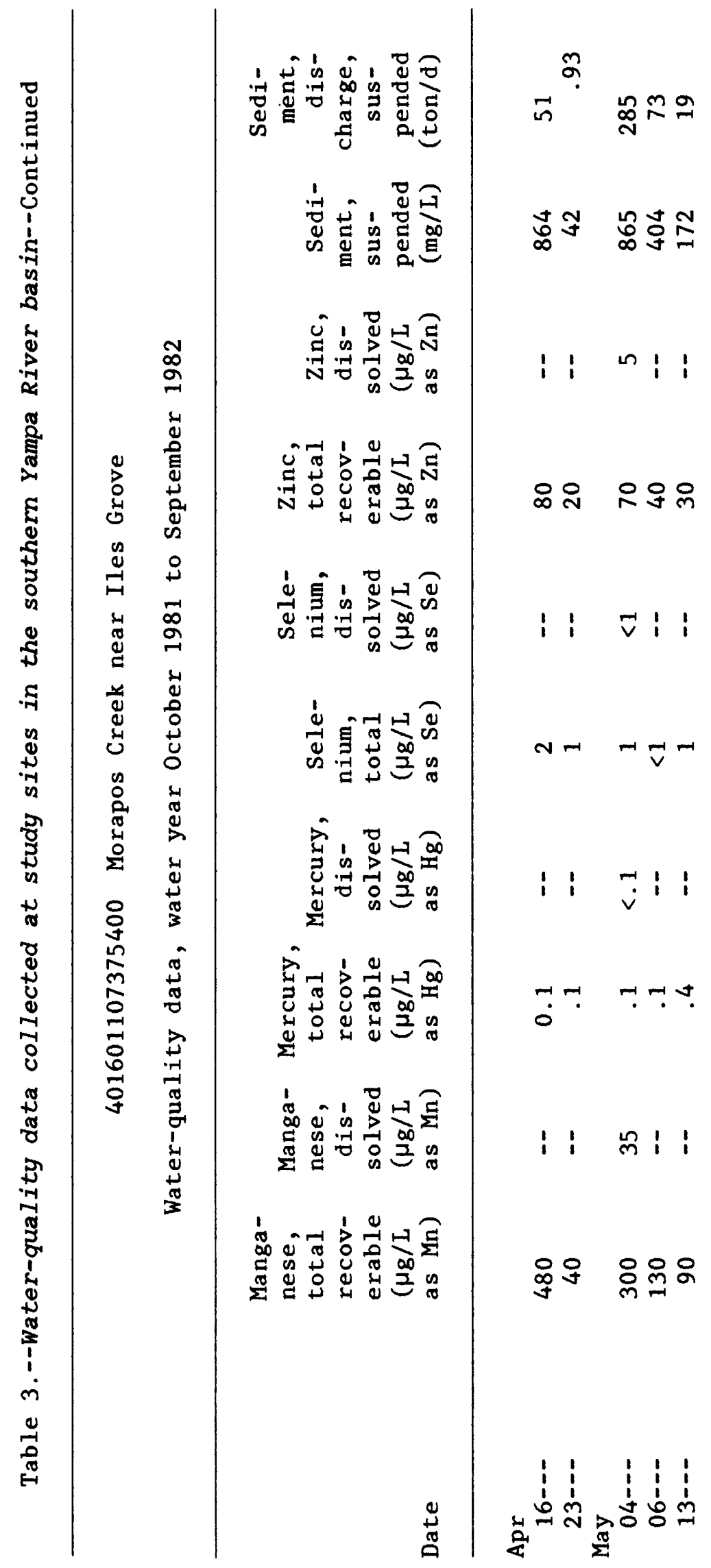




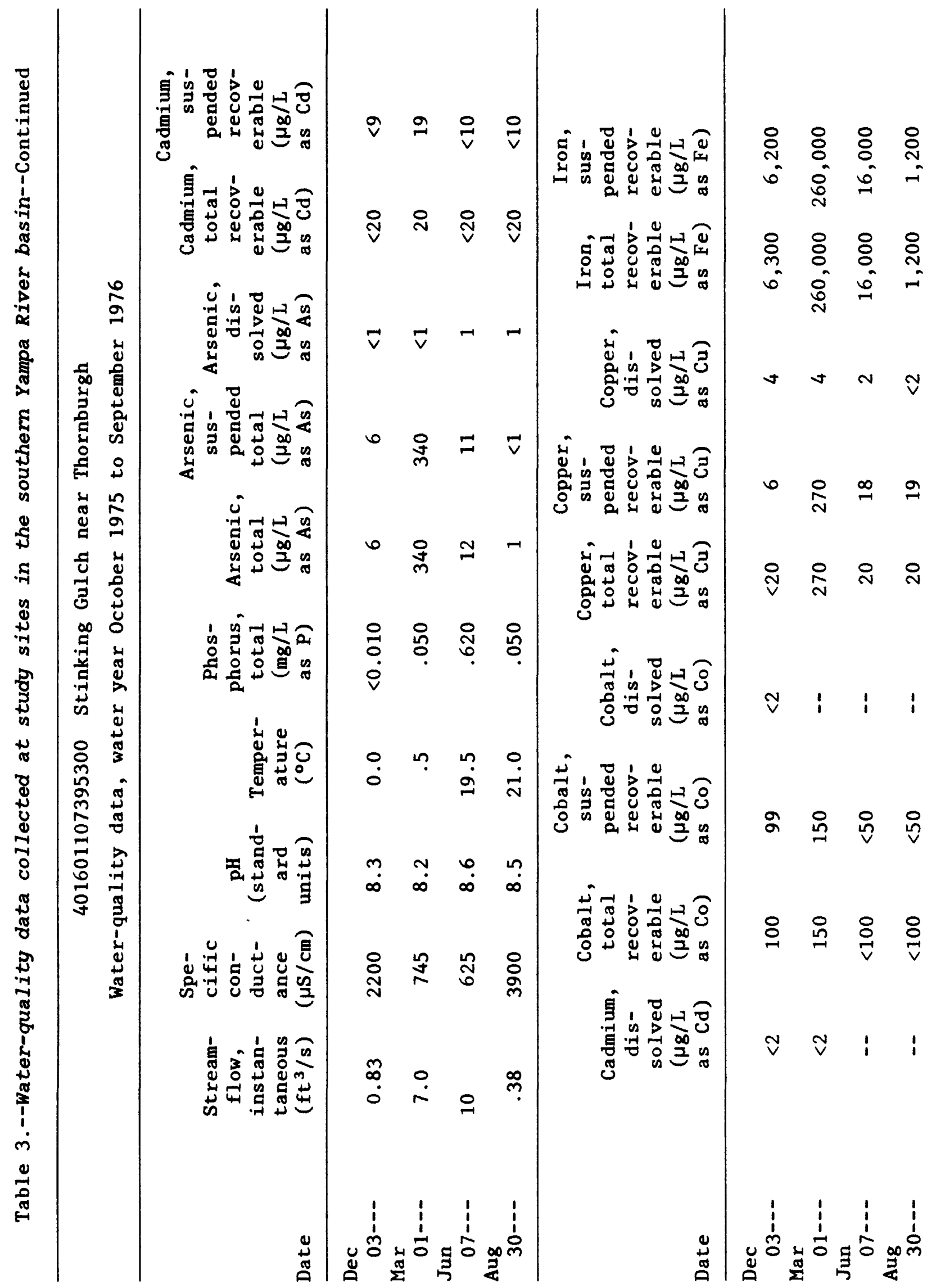




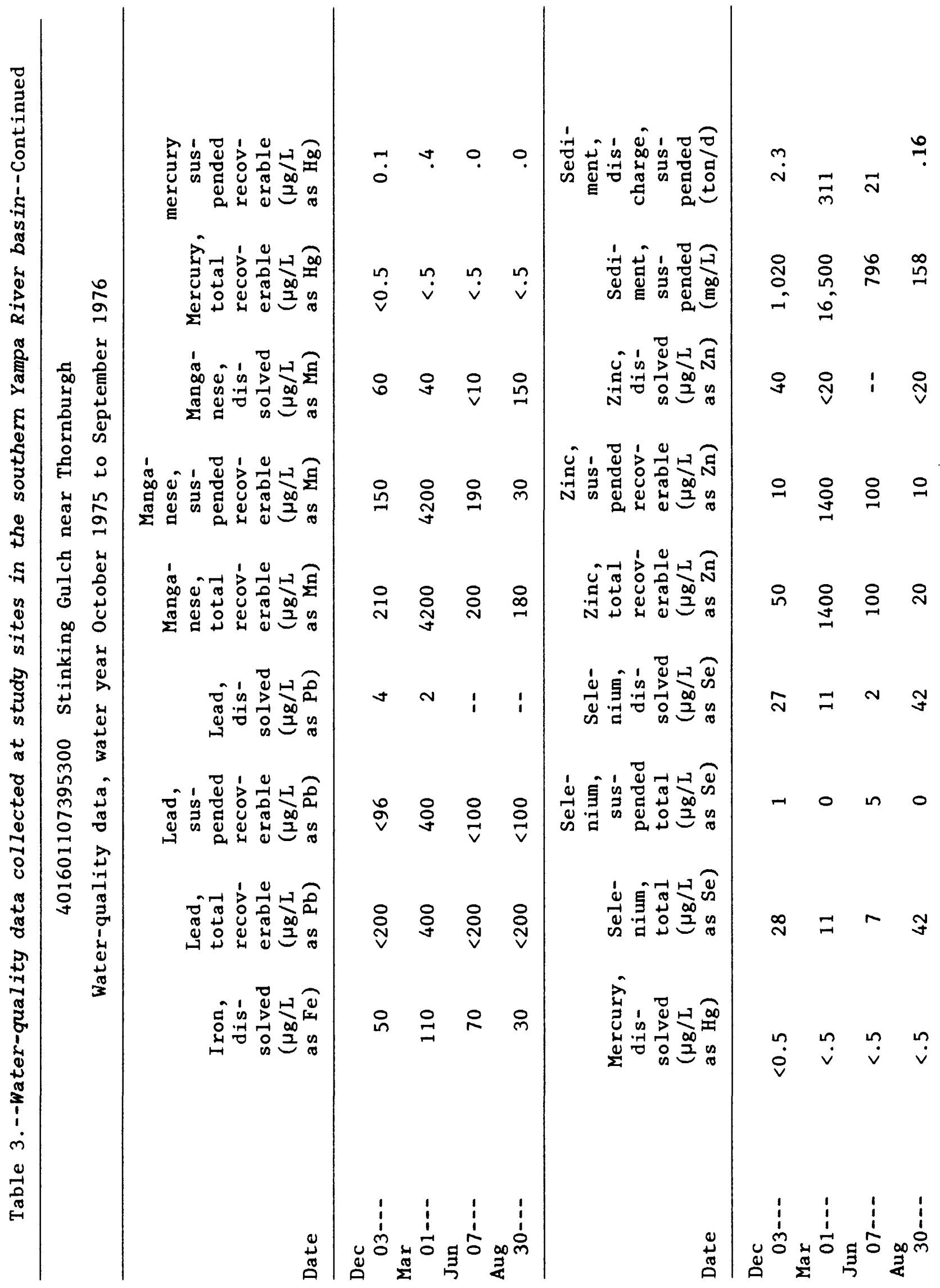




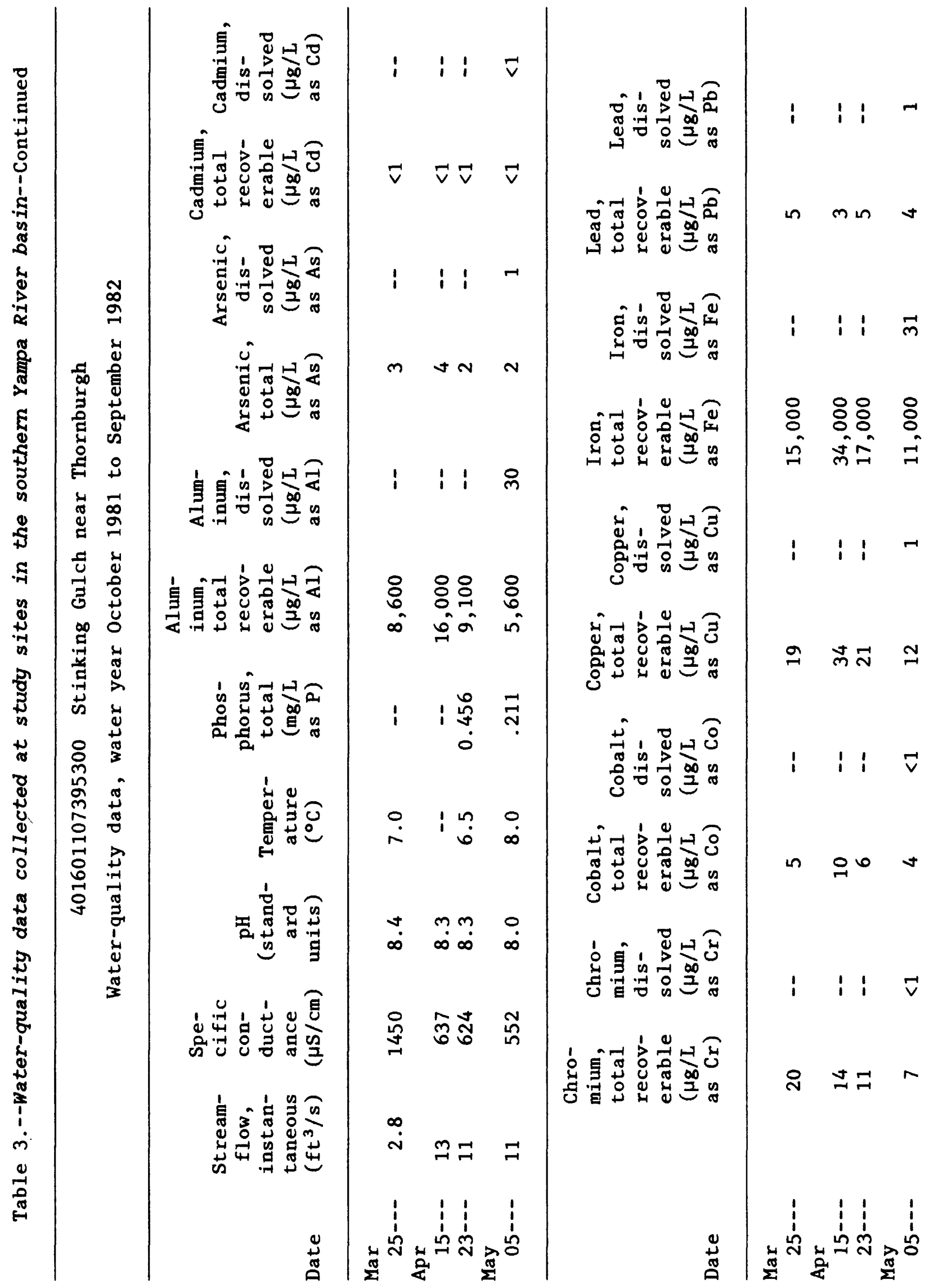




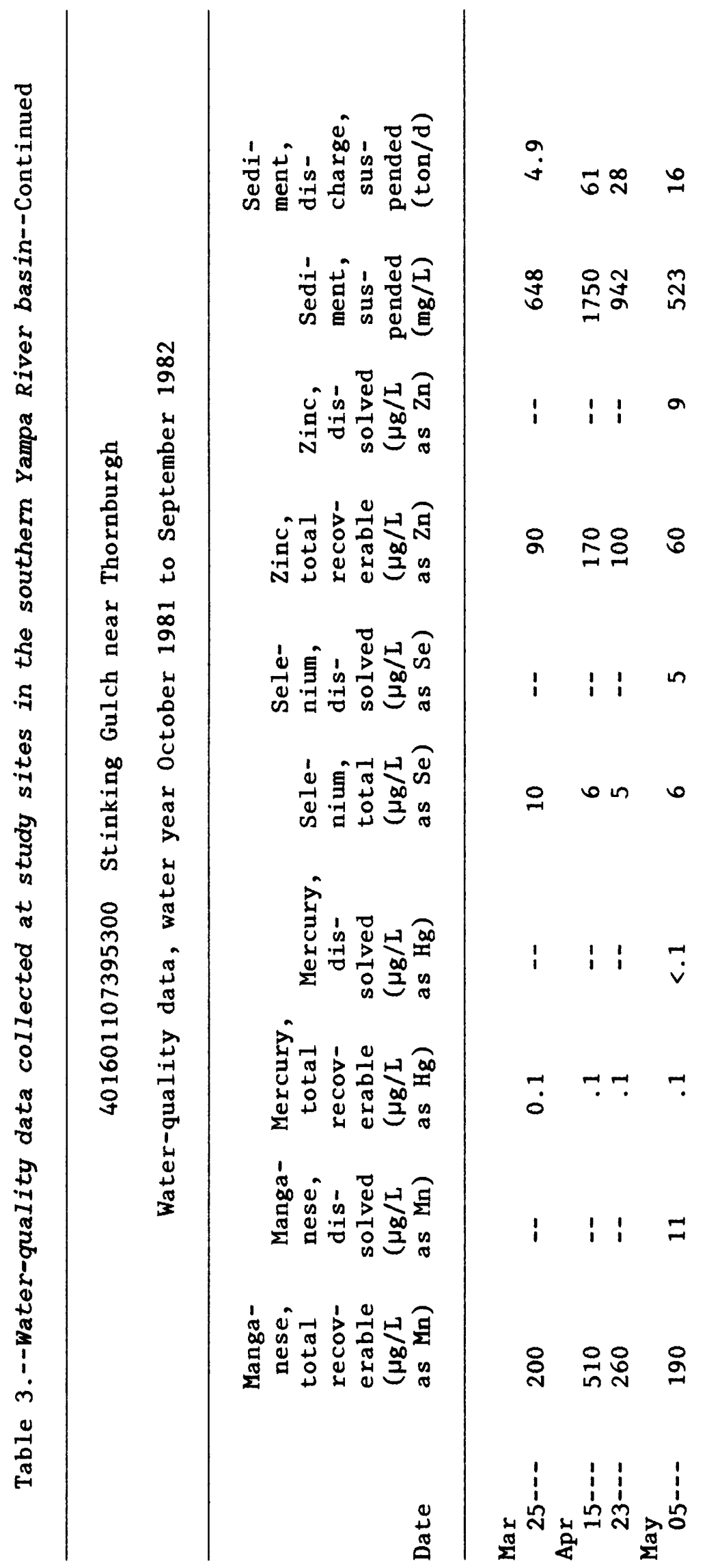




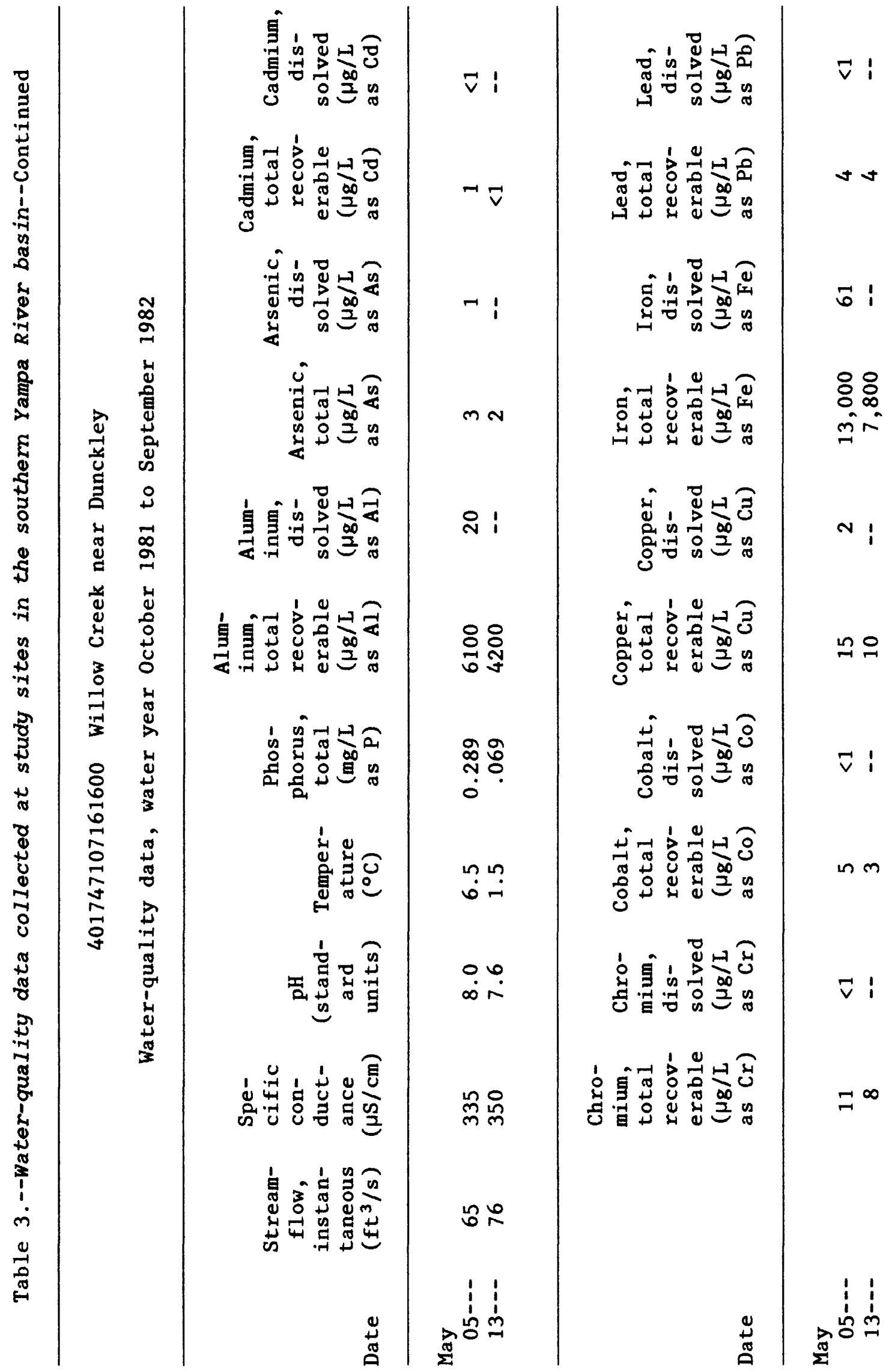




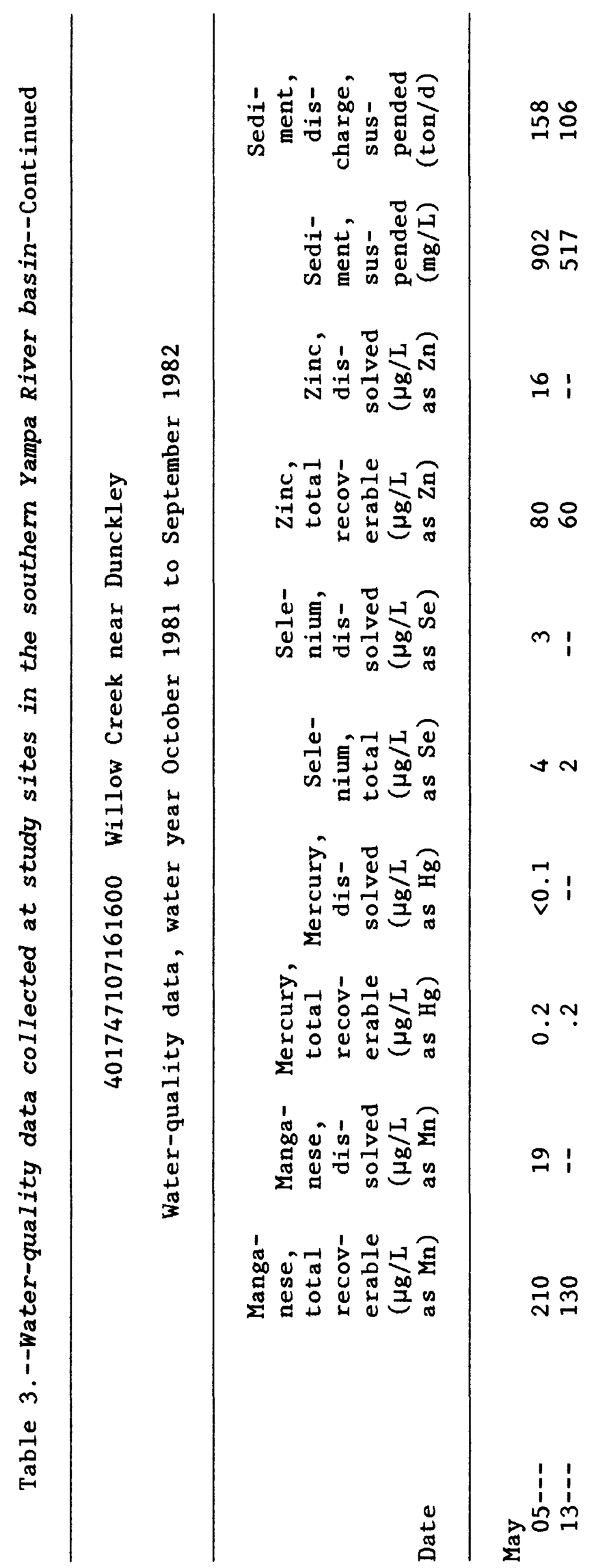




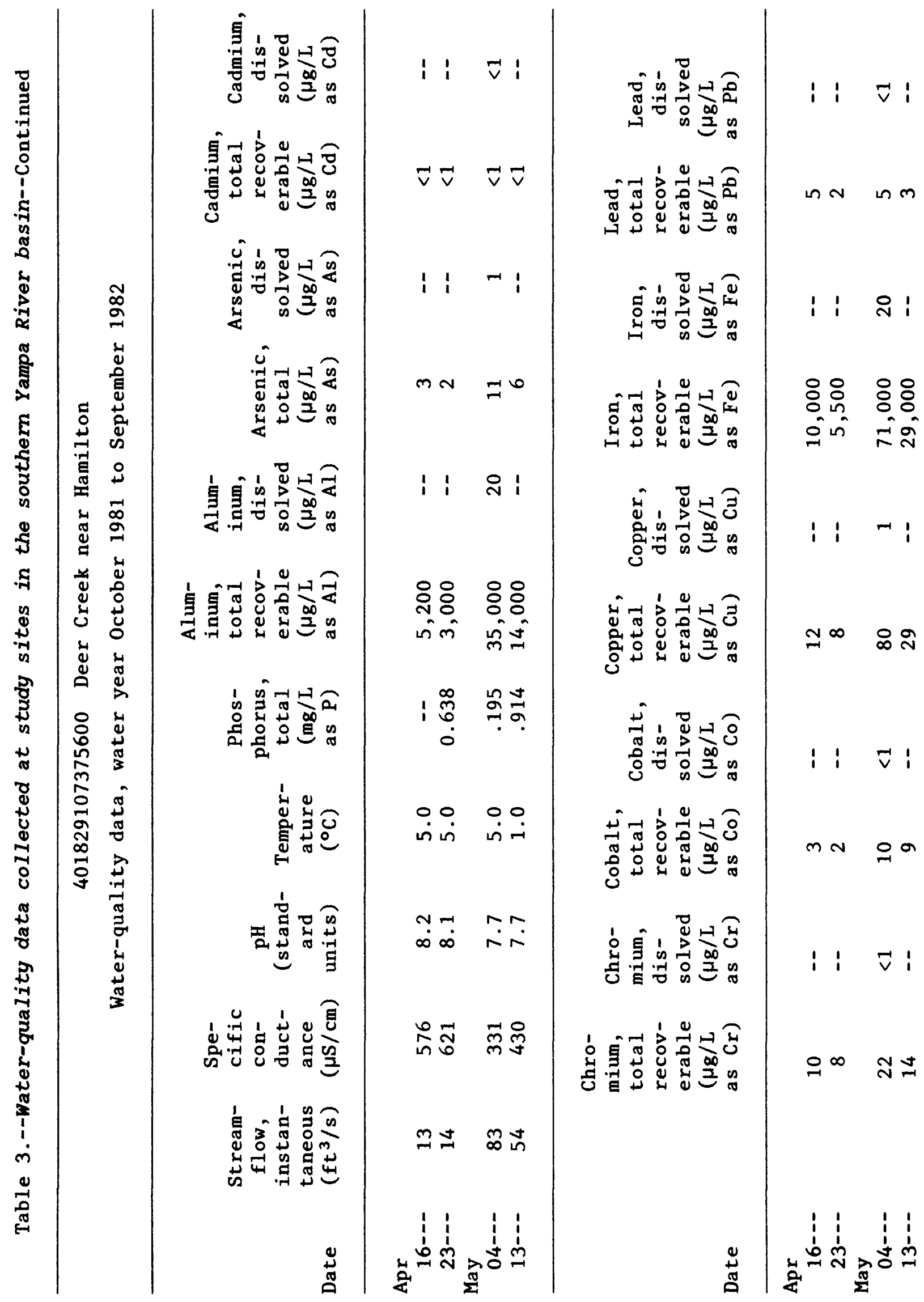




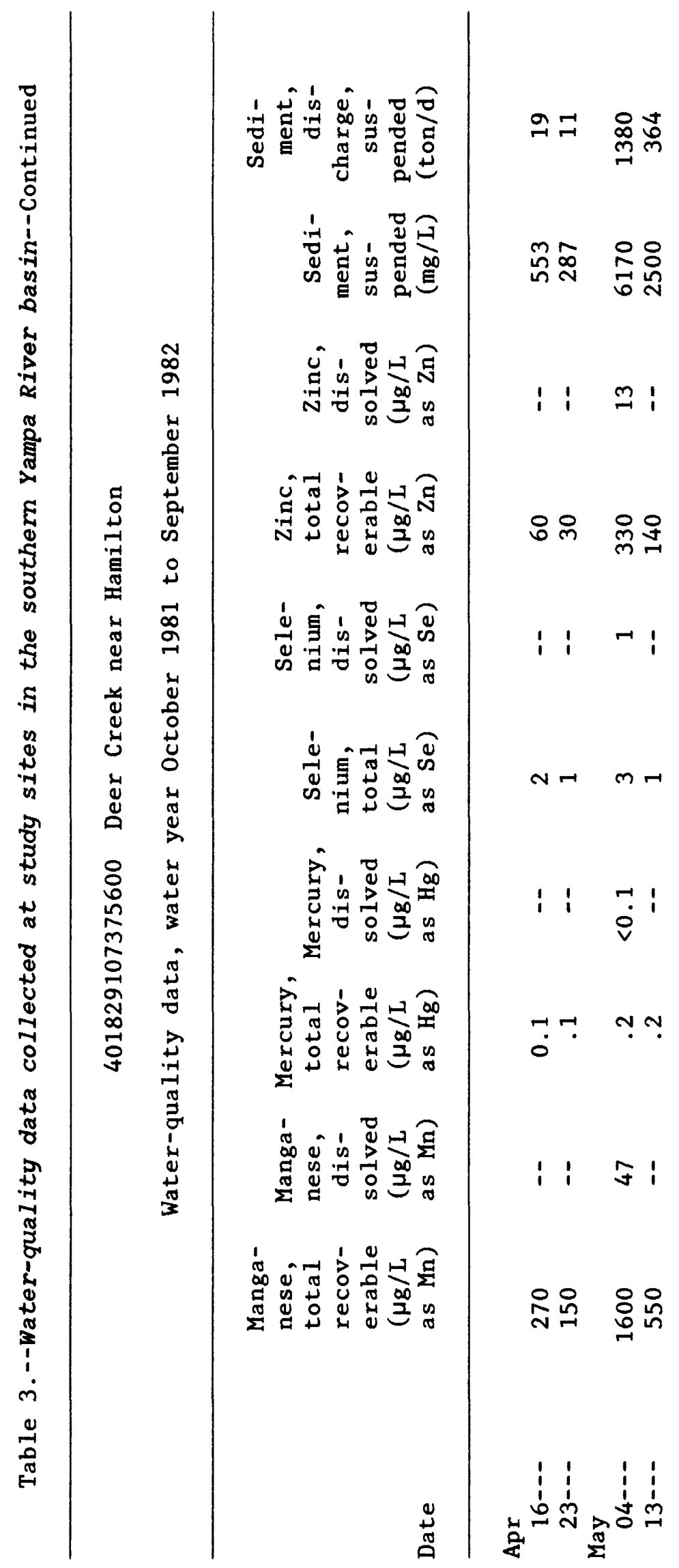




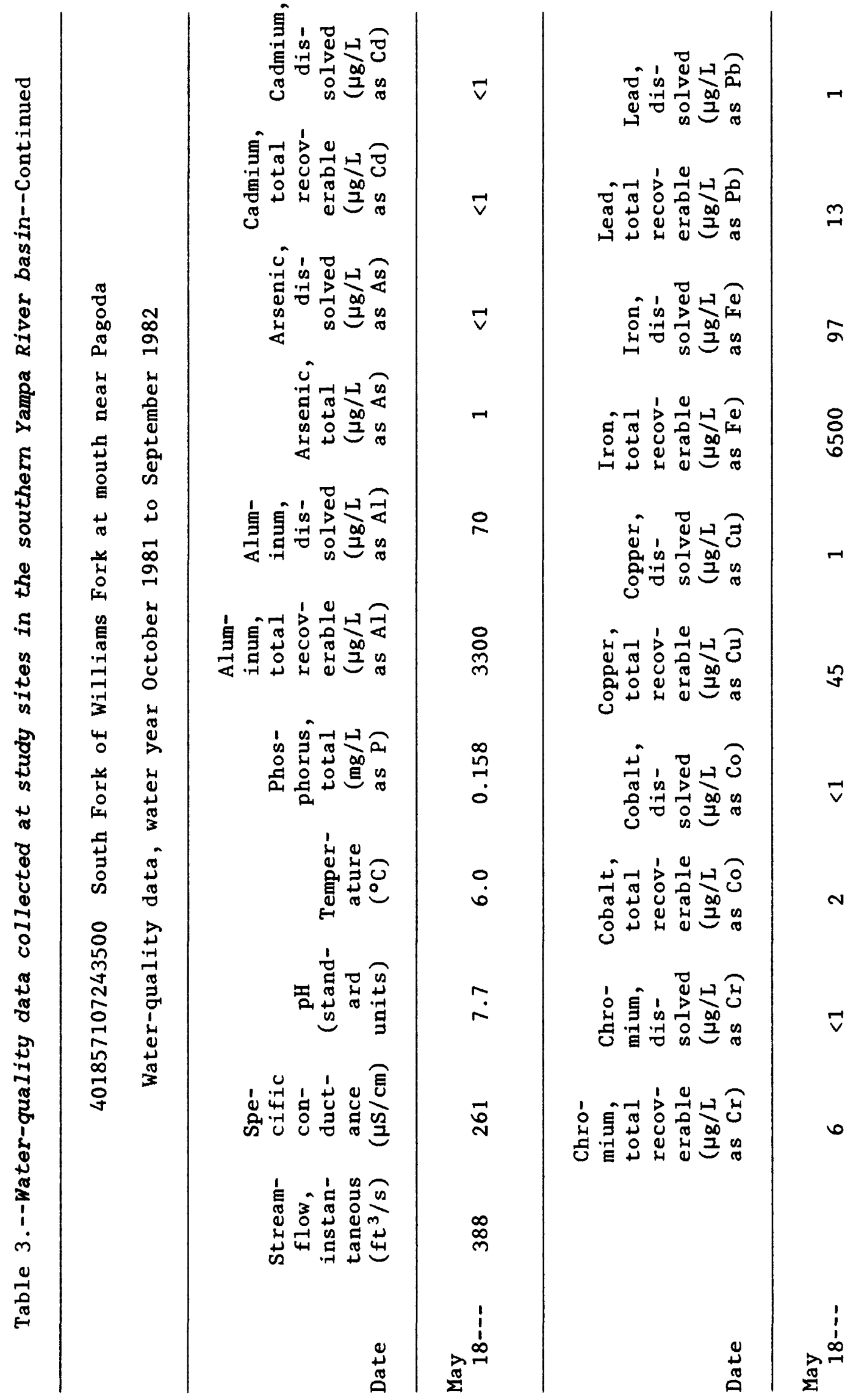




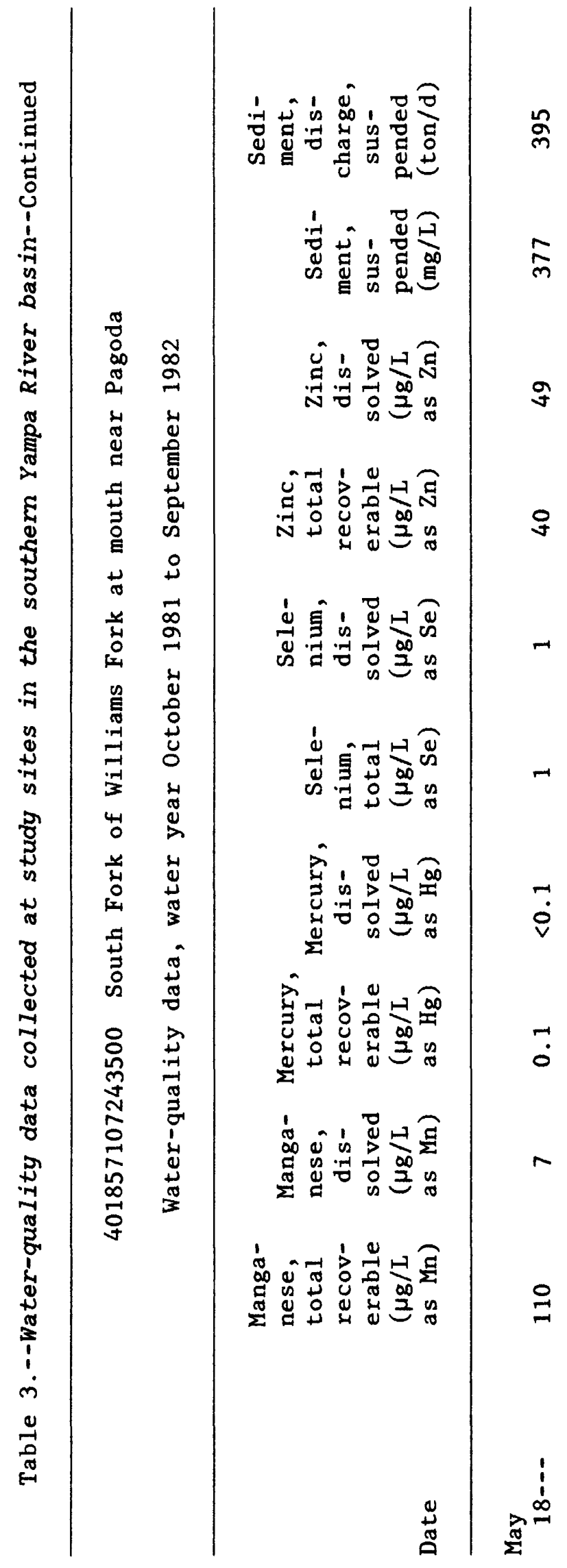




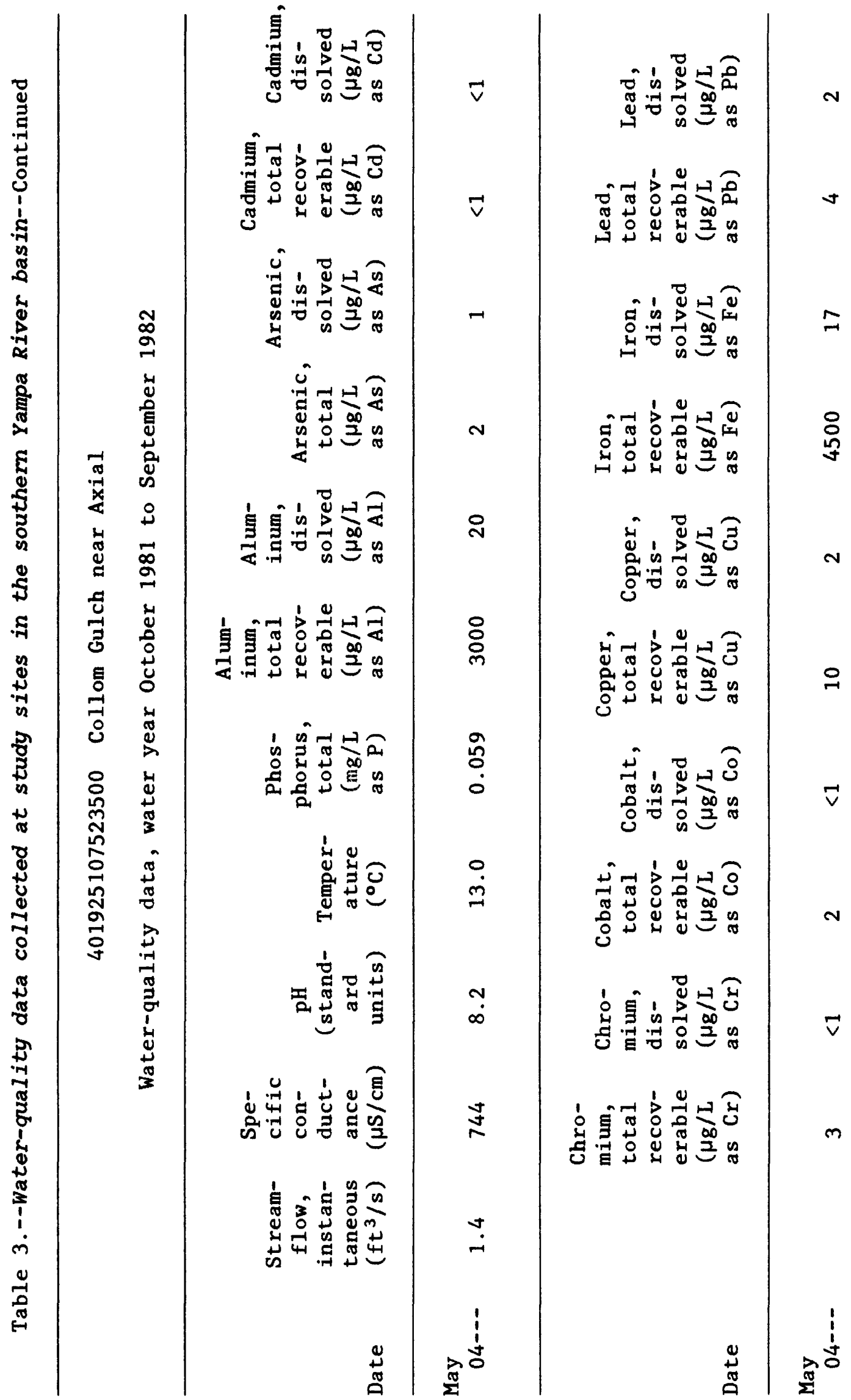




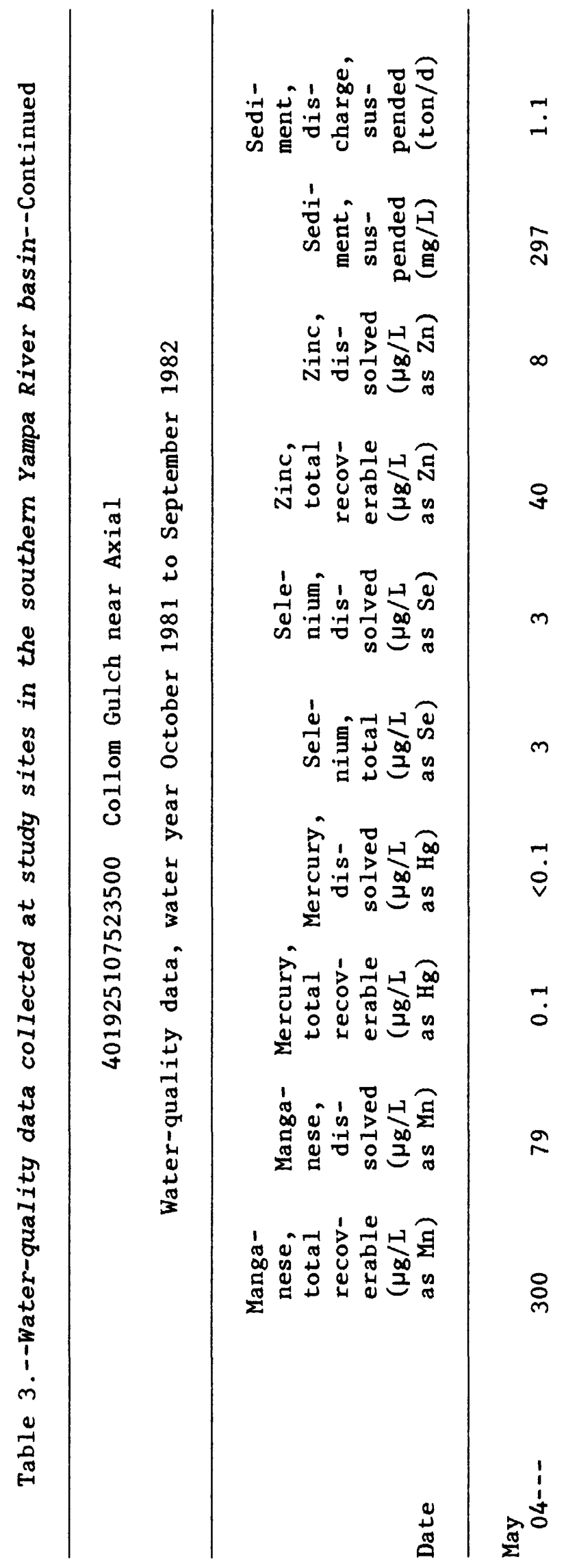




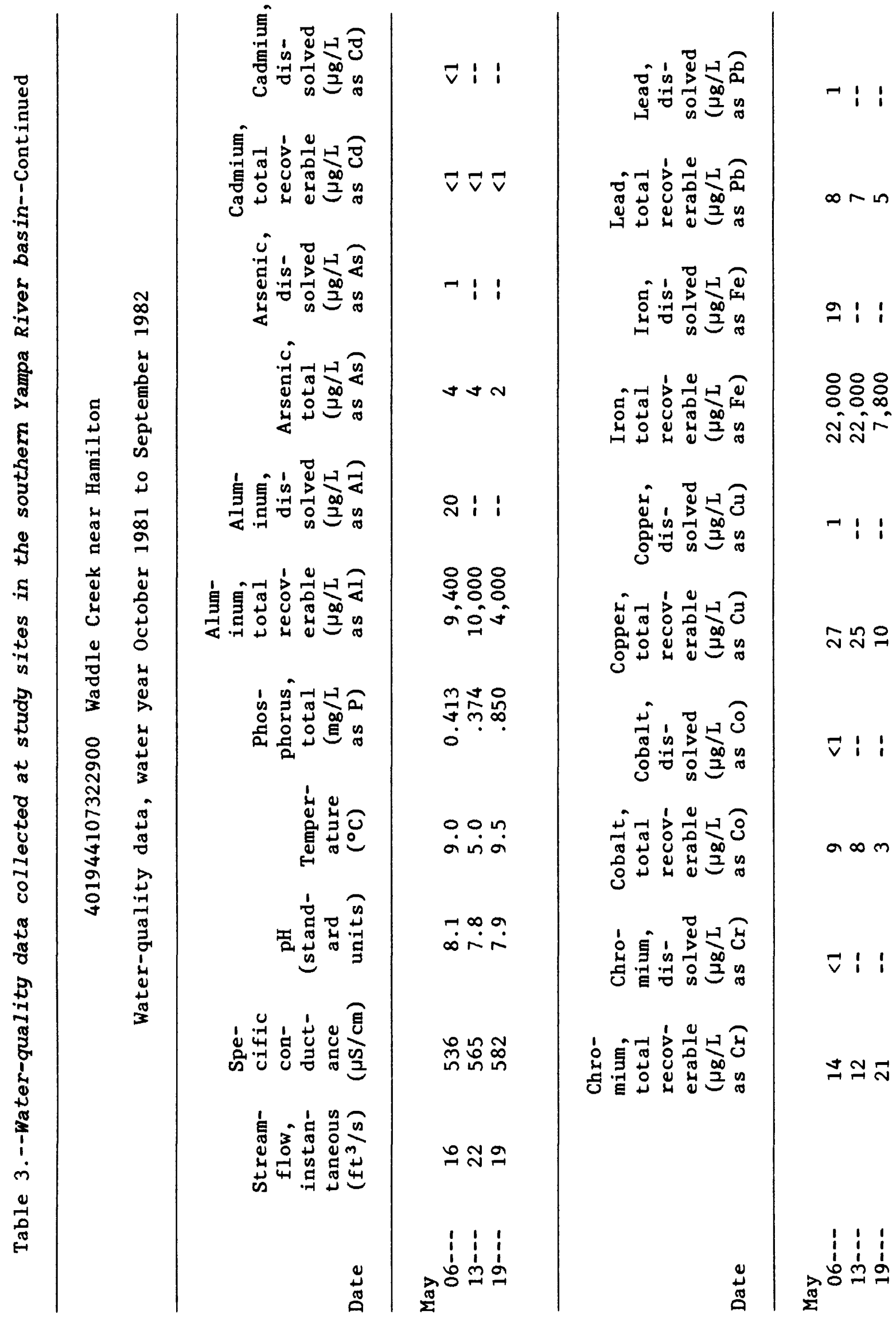




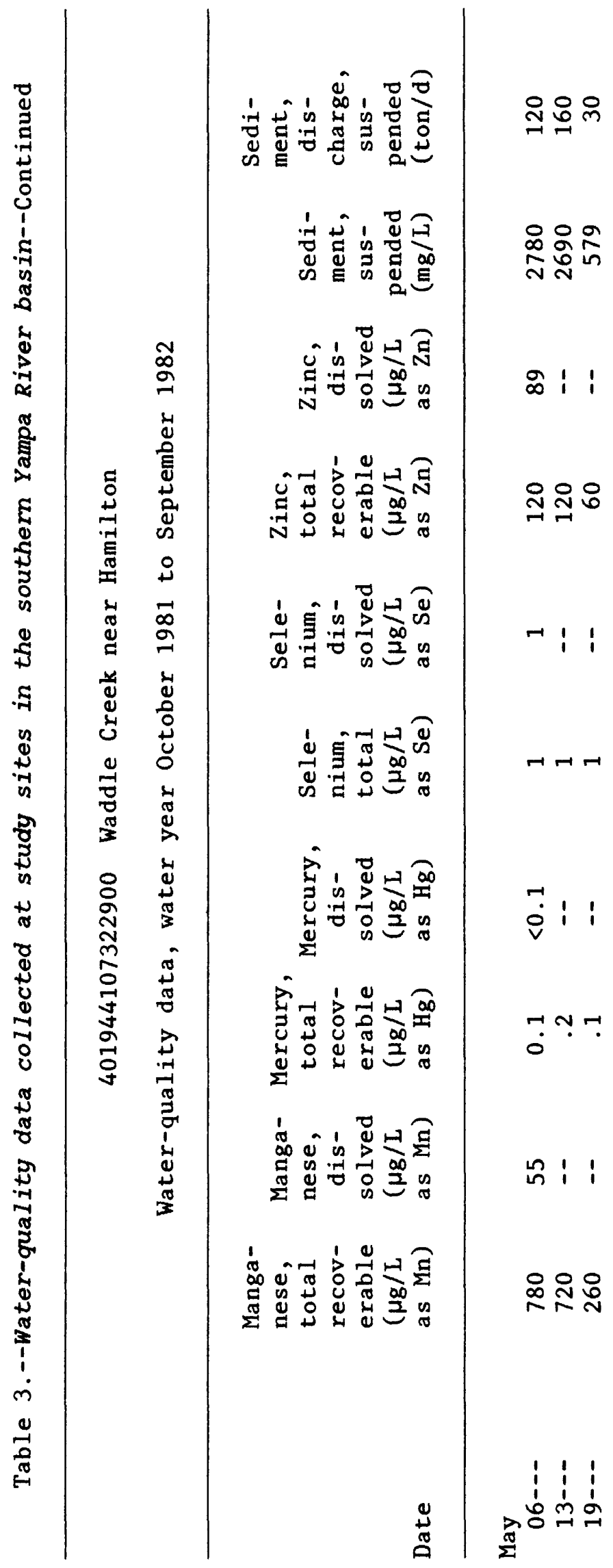




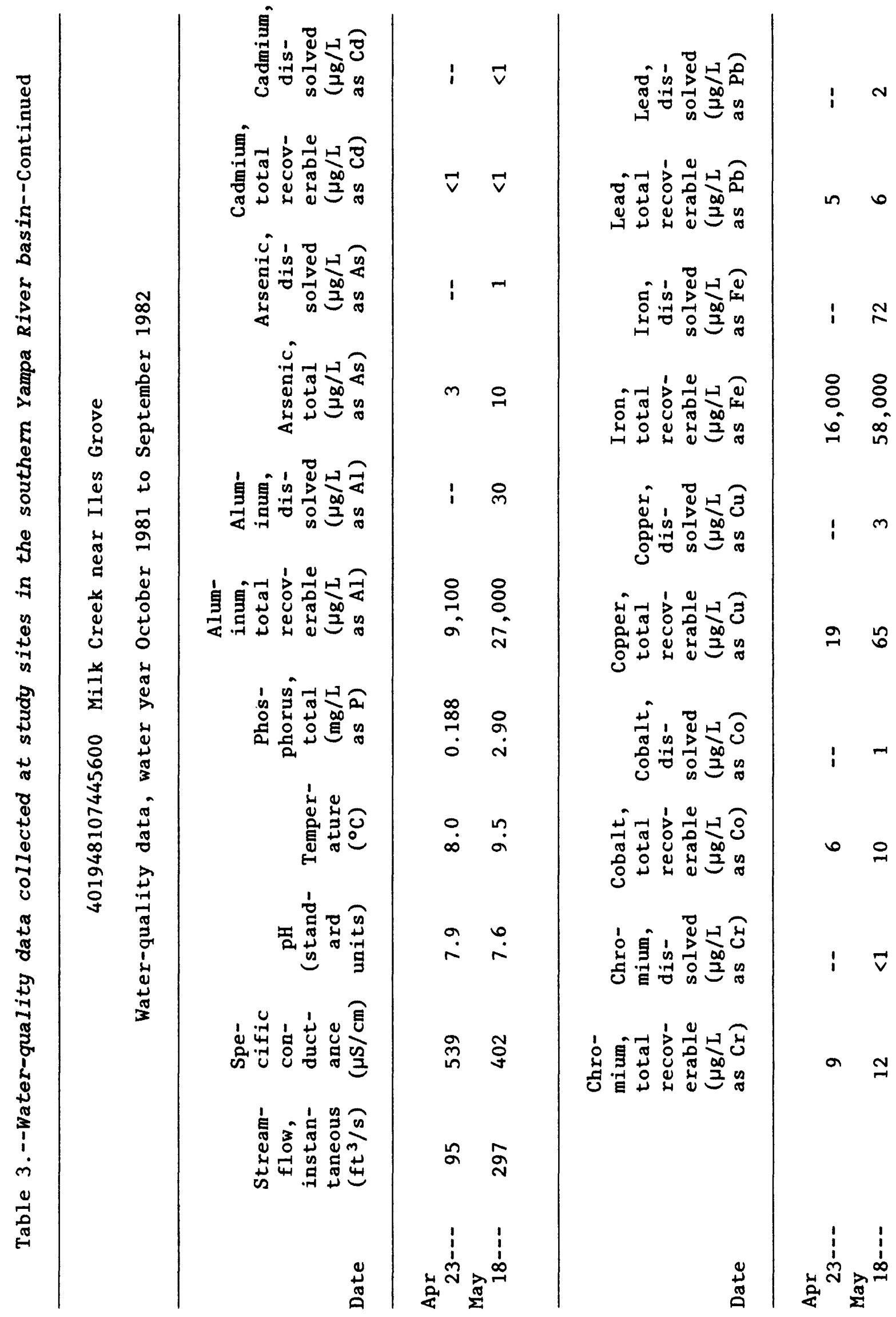




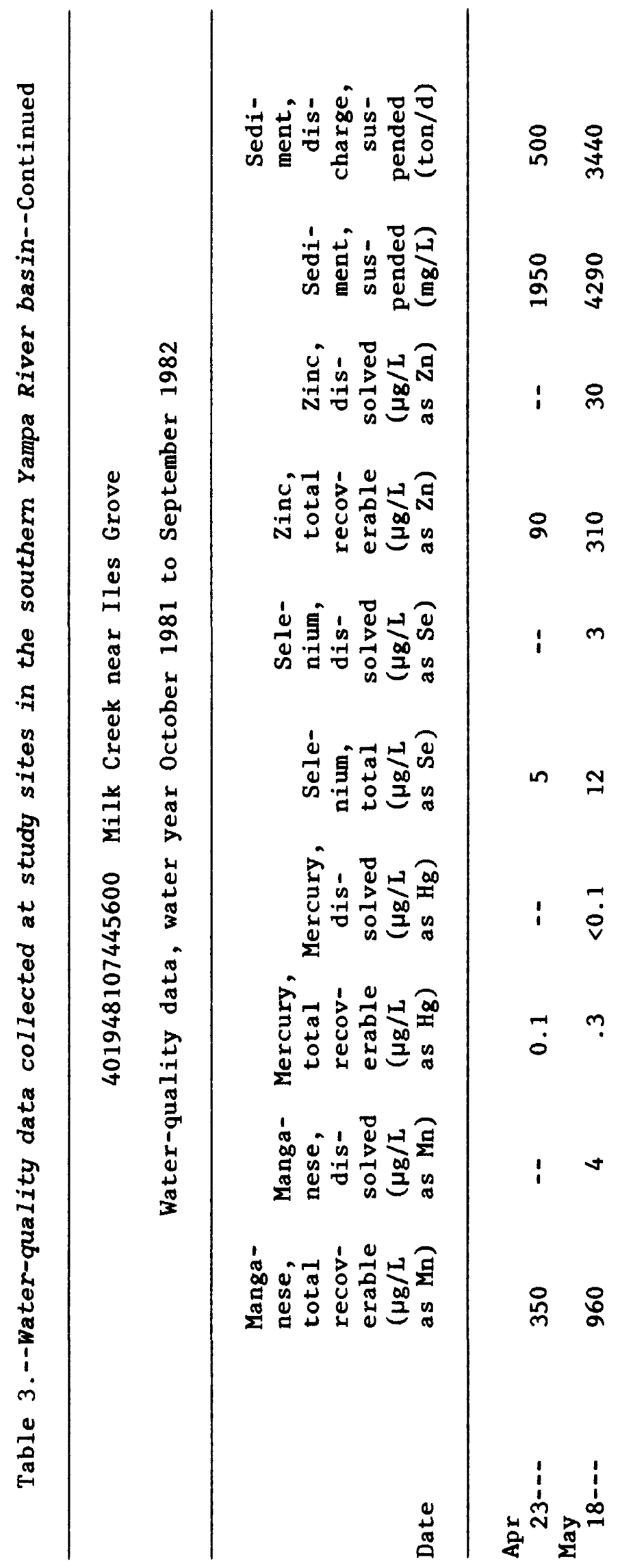




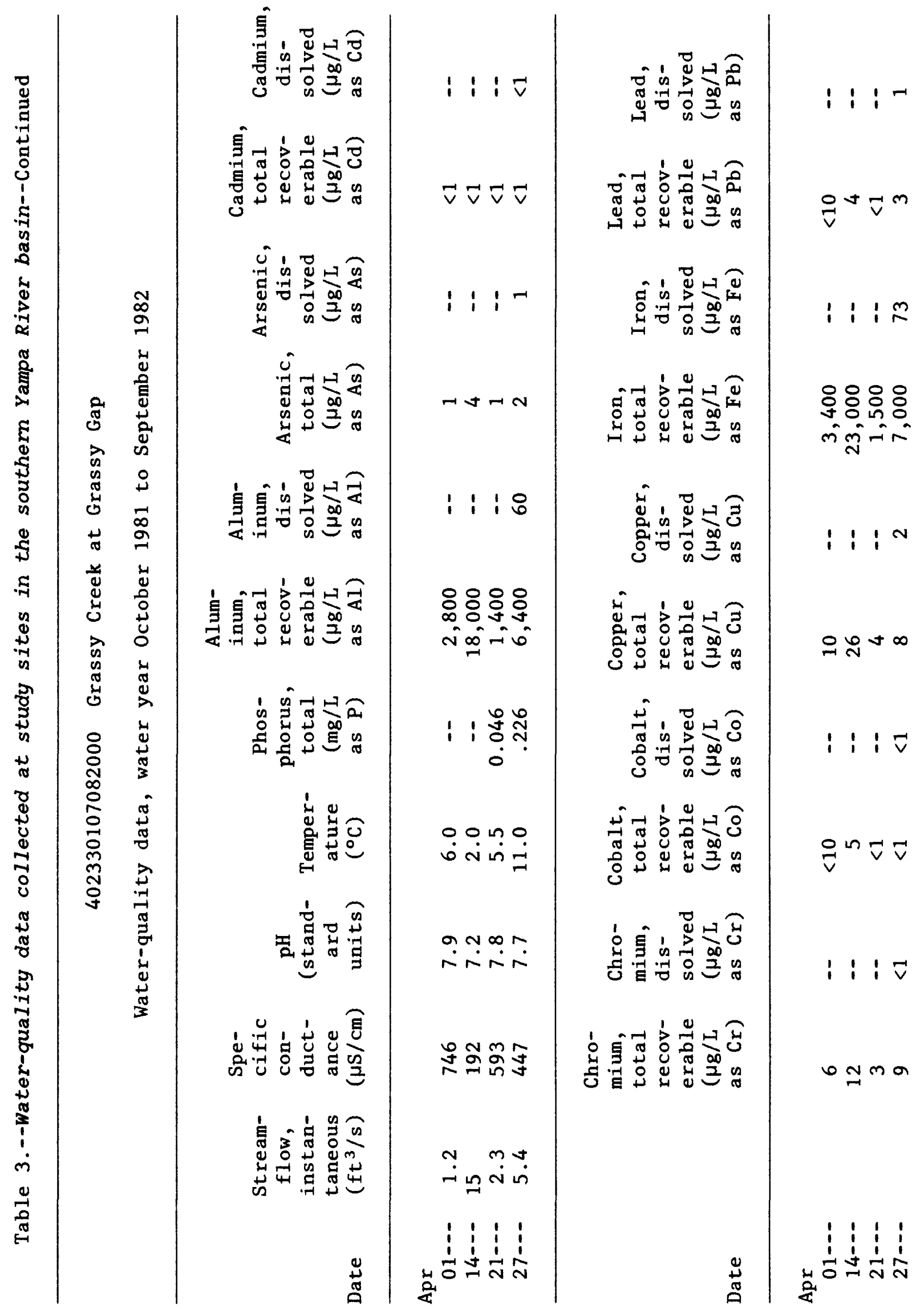




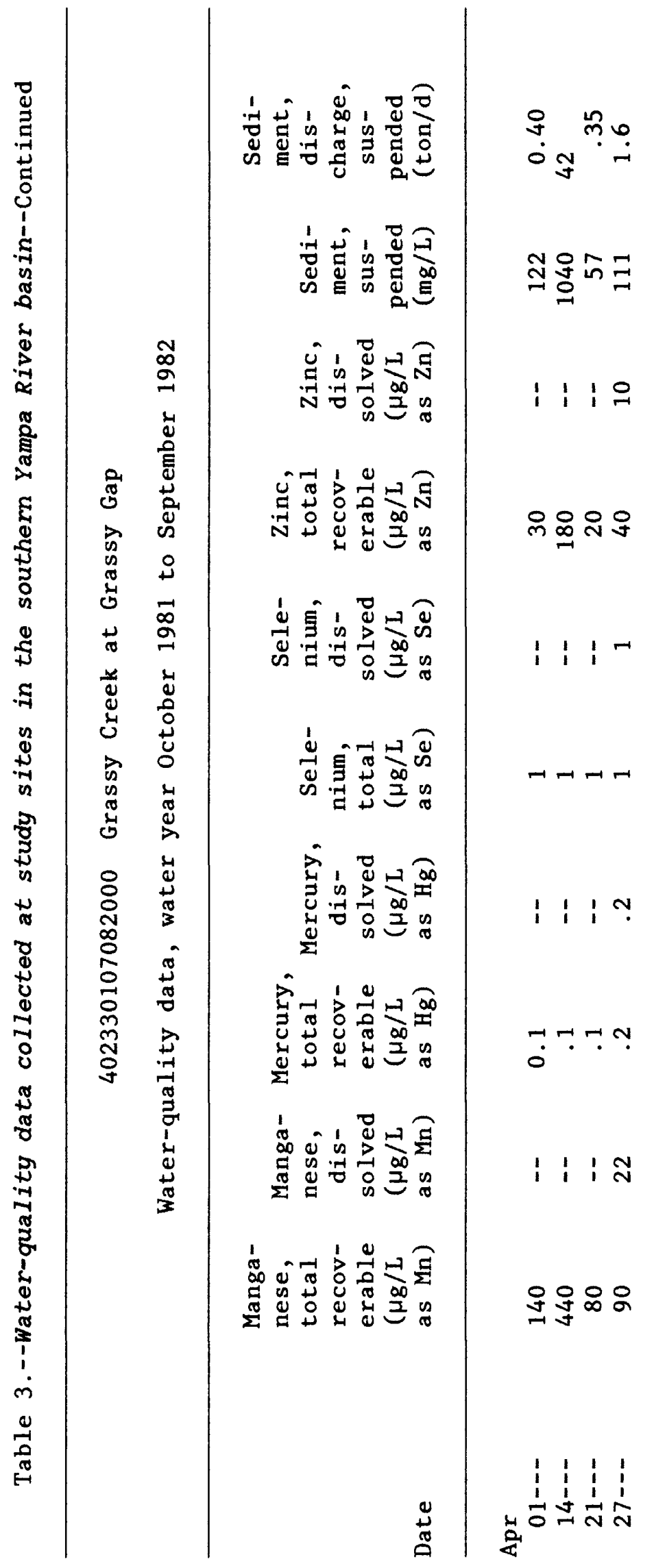




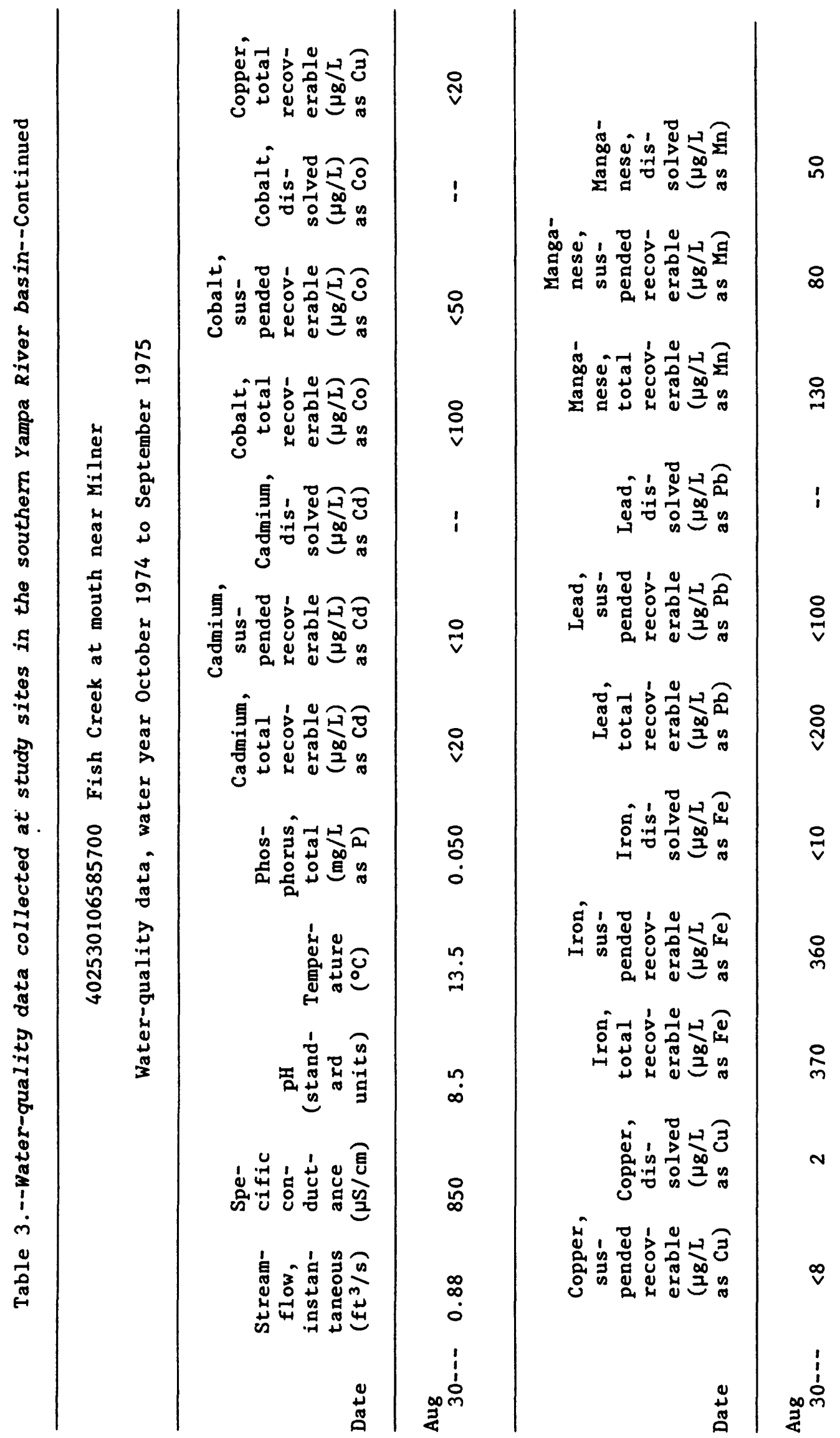




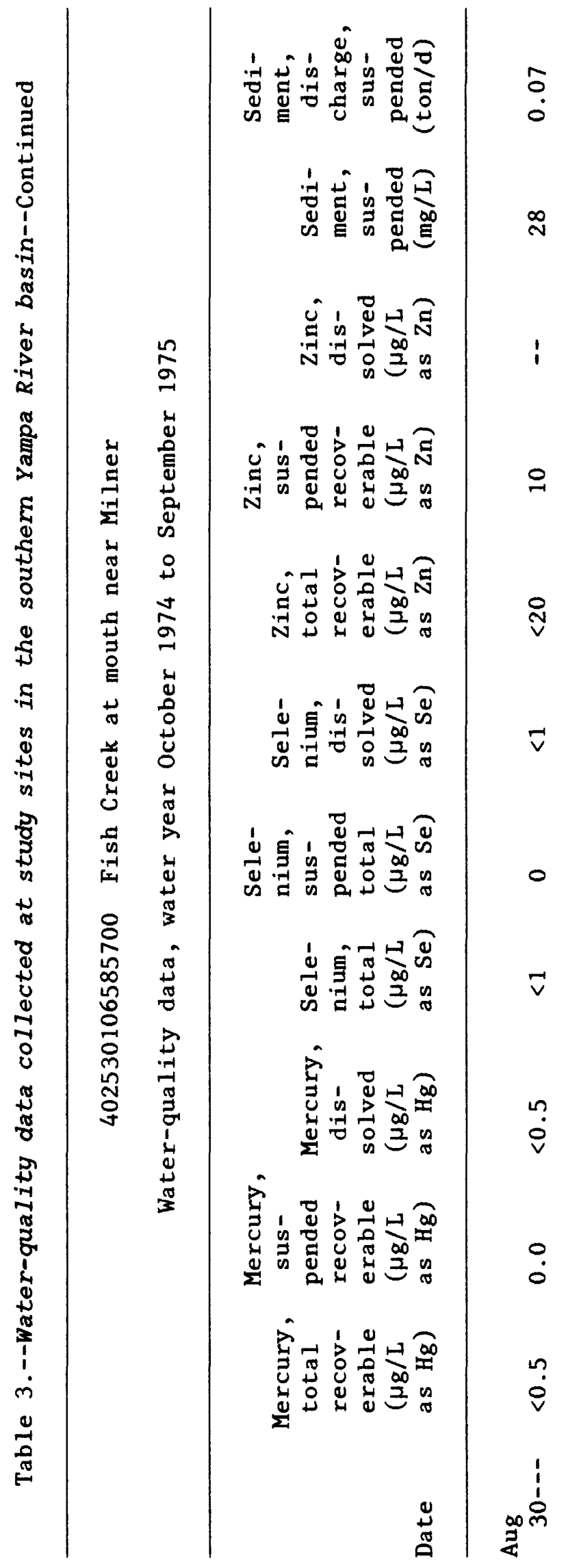




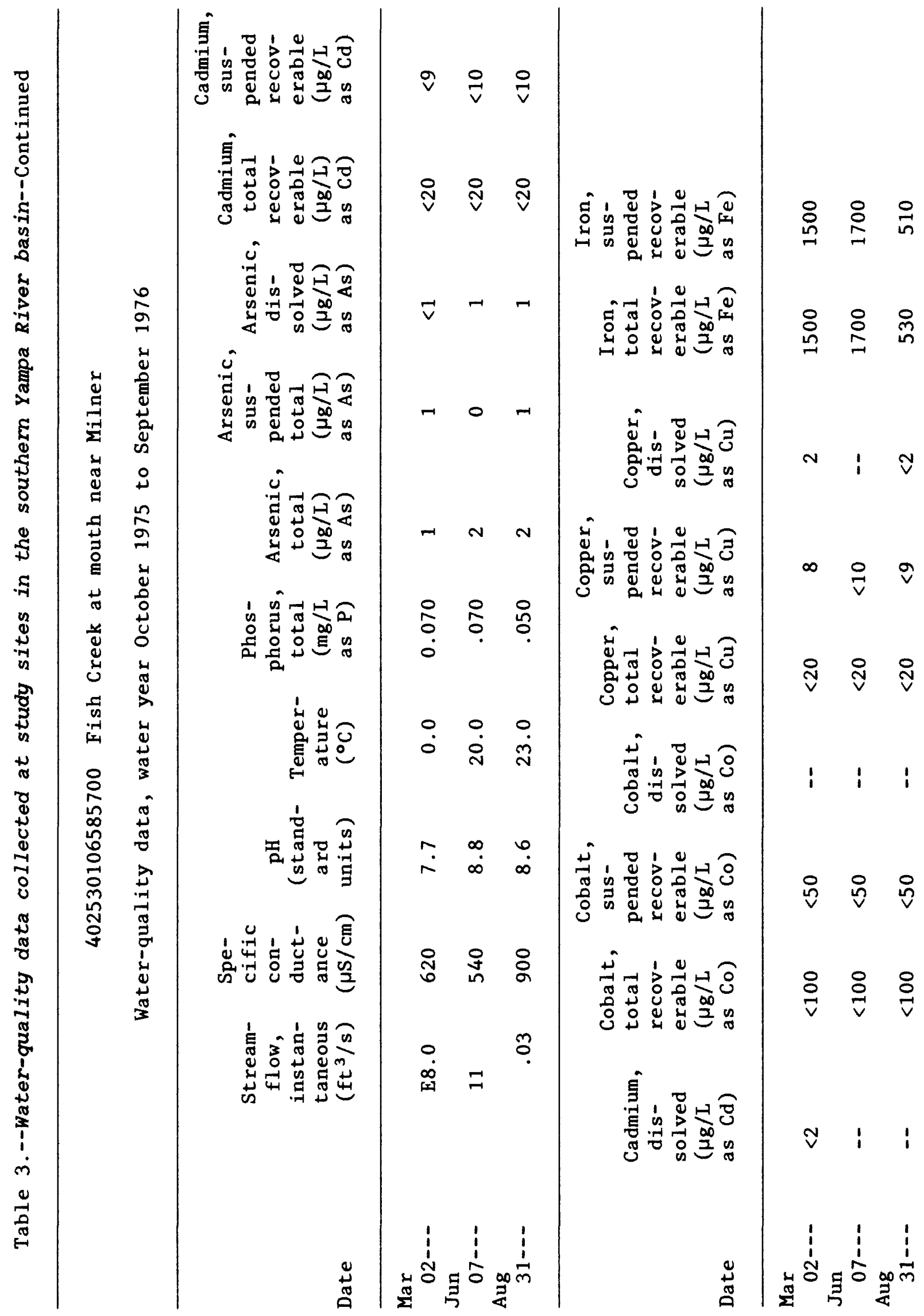




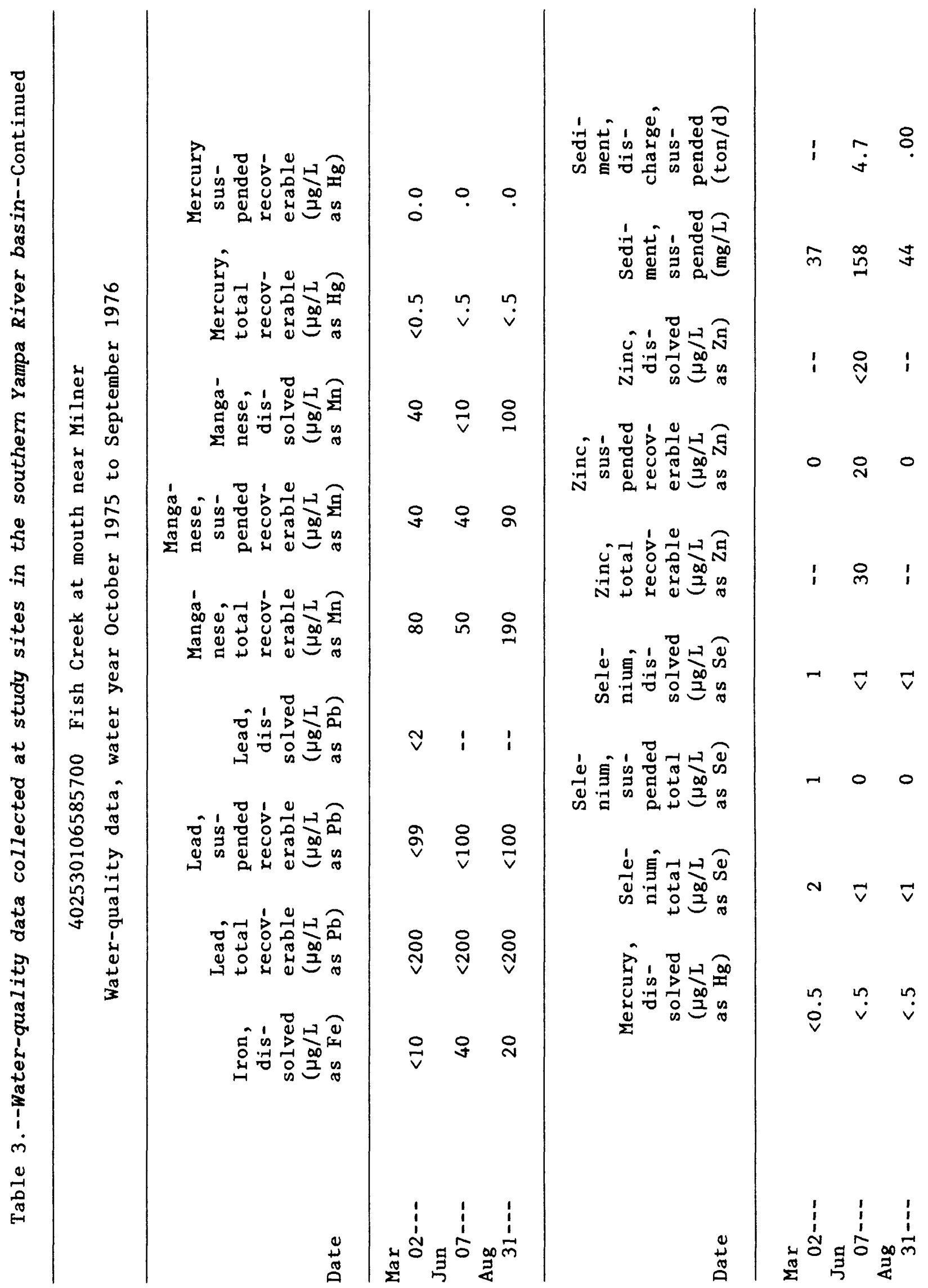




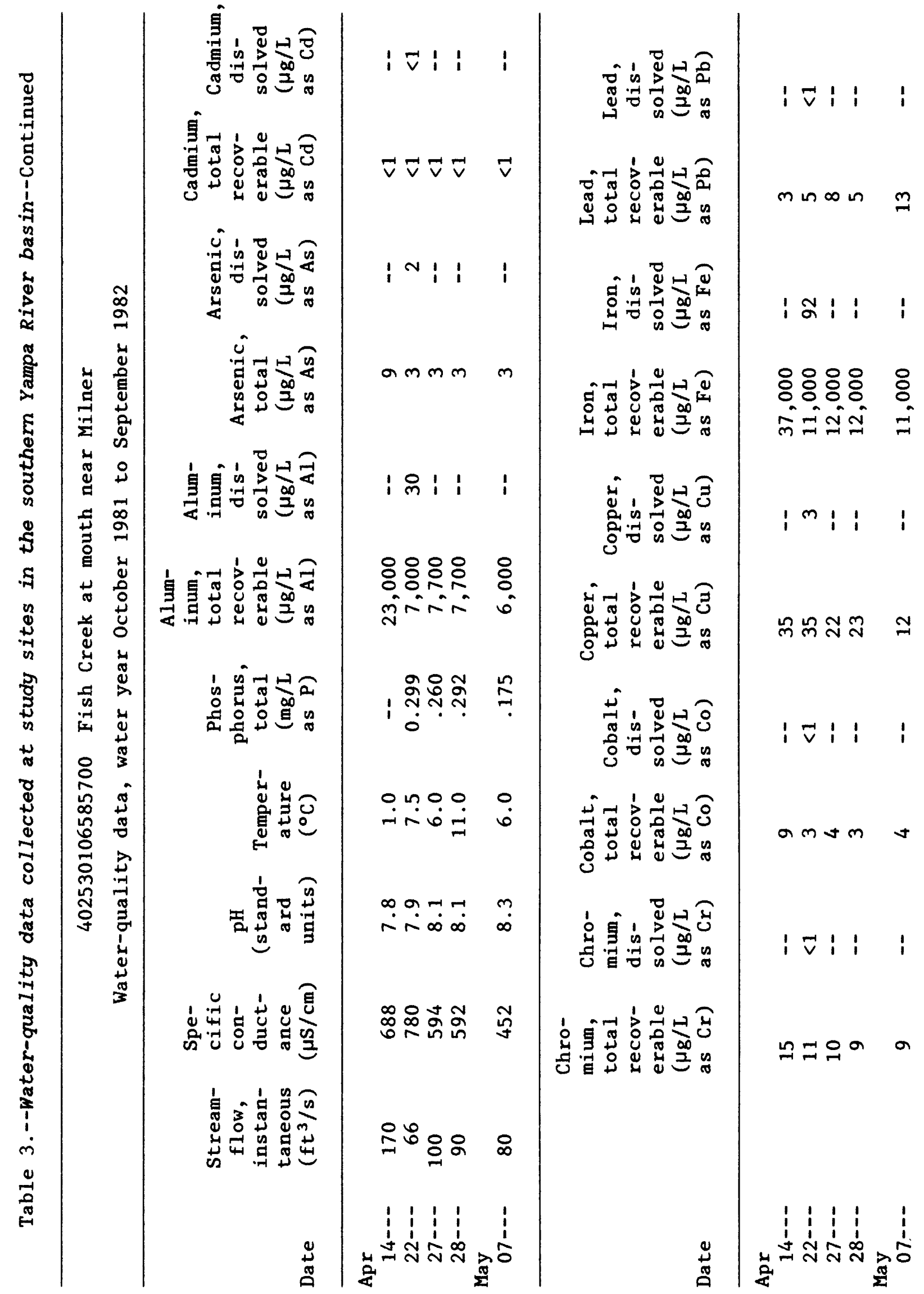




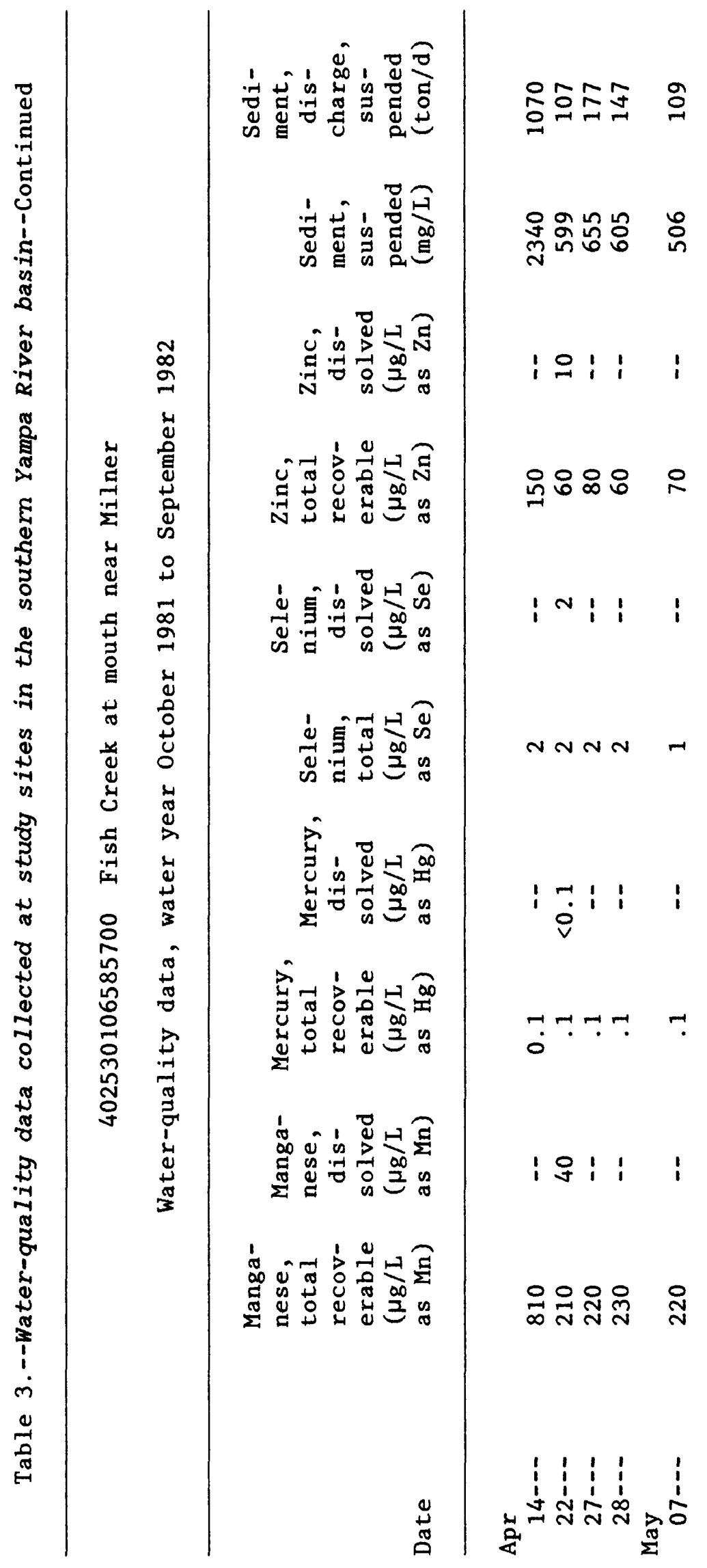




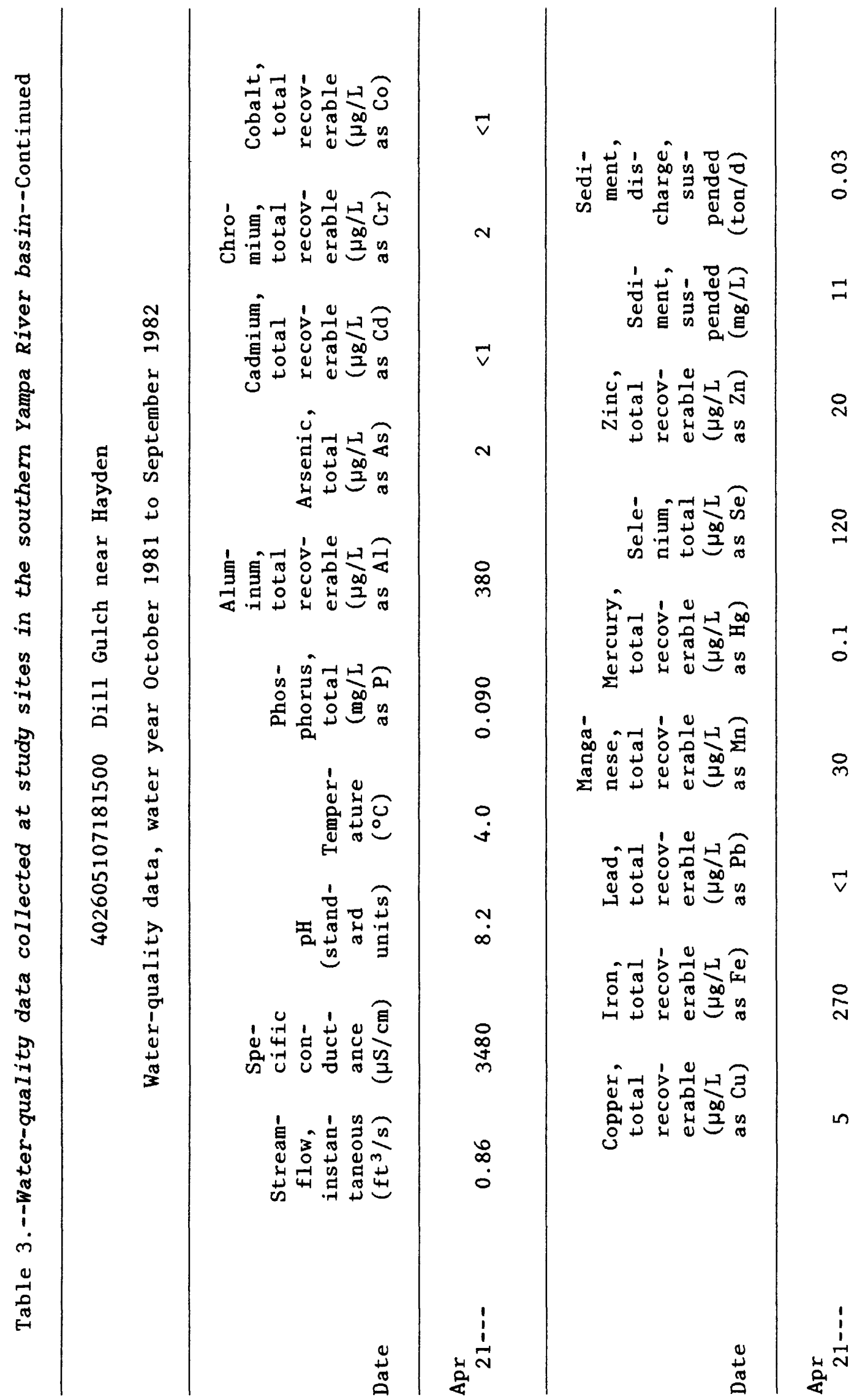




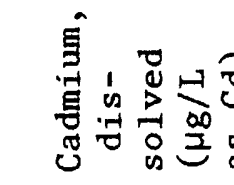

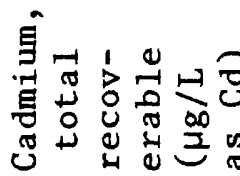

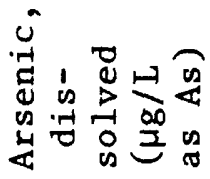

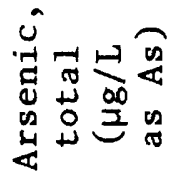

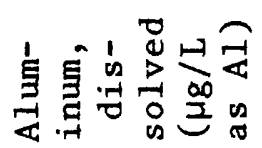

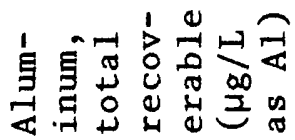

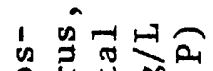

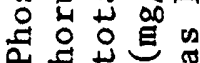

D.

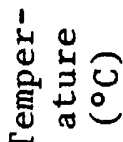

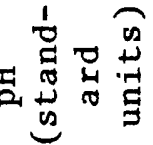

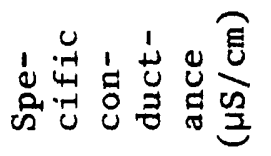

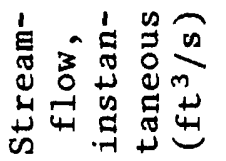

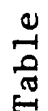

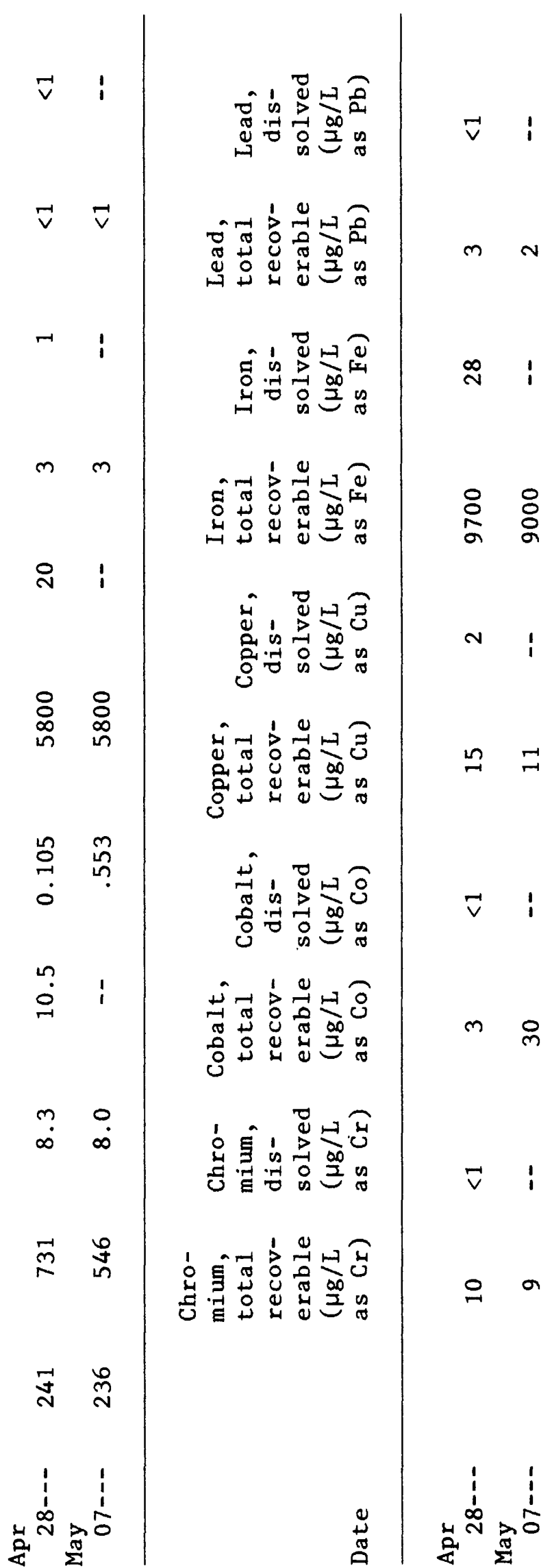




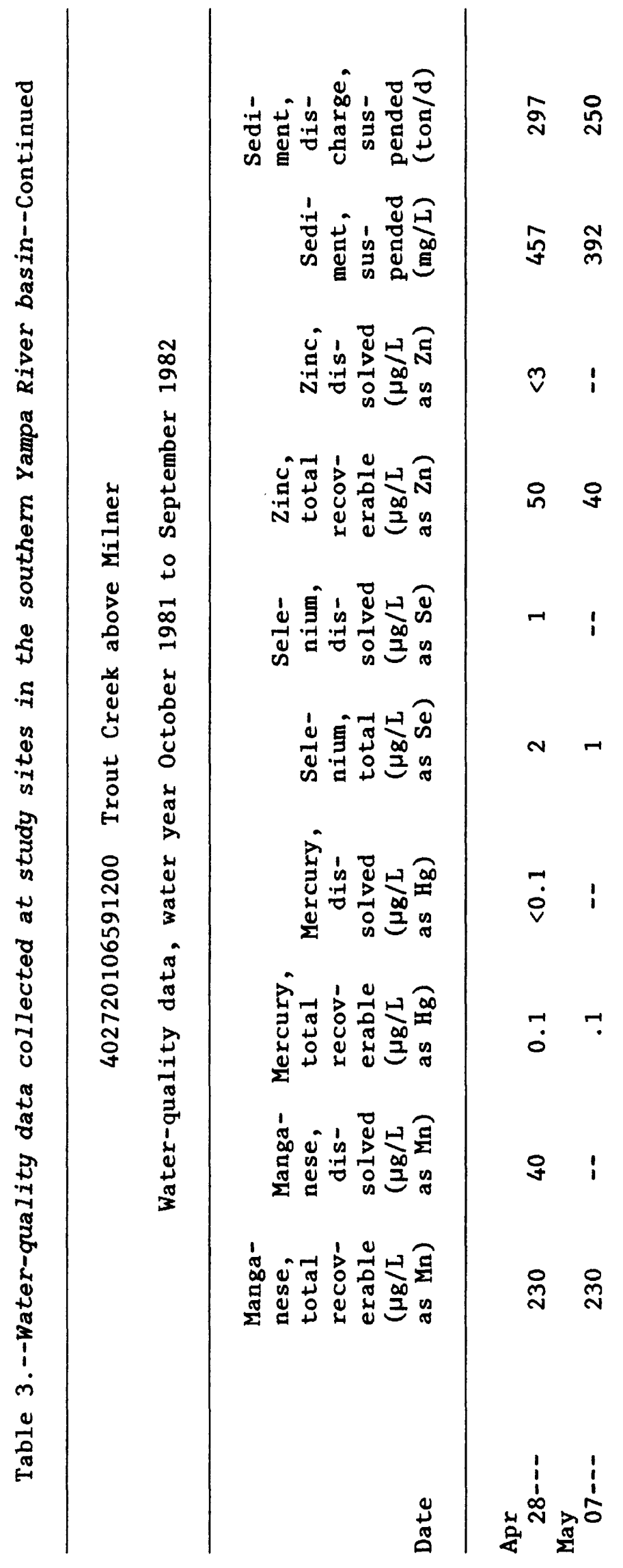




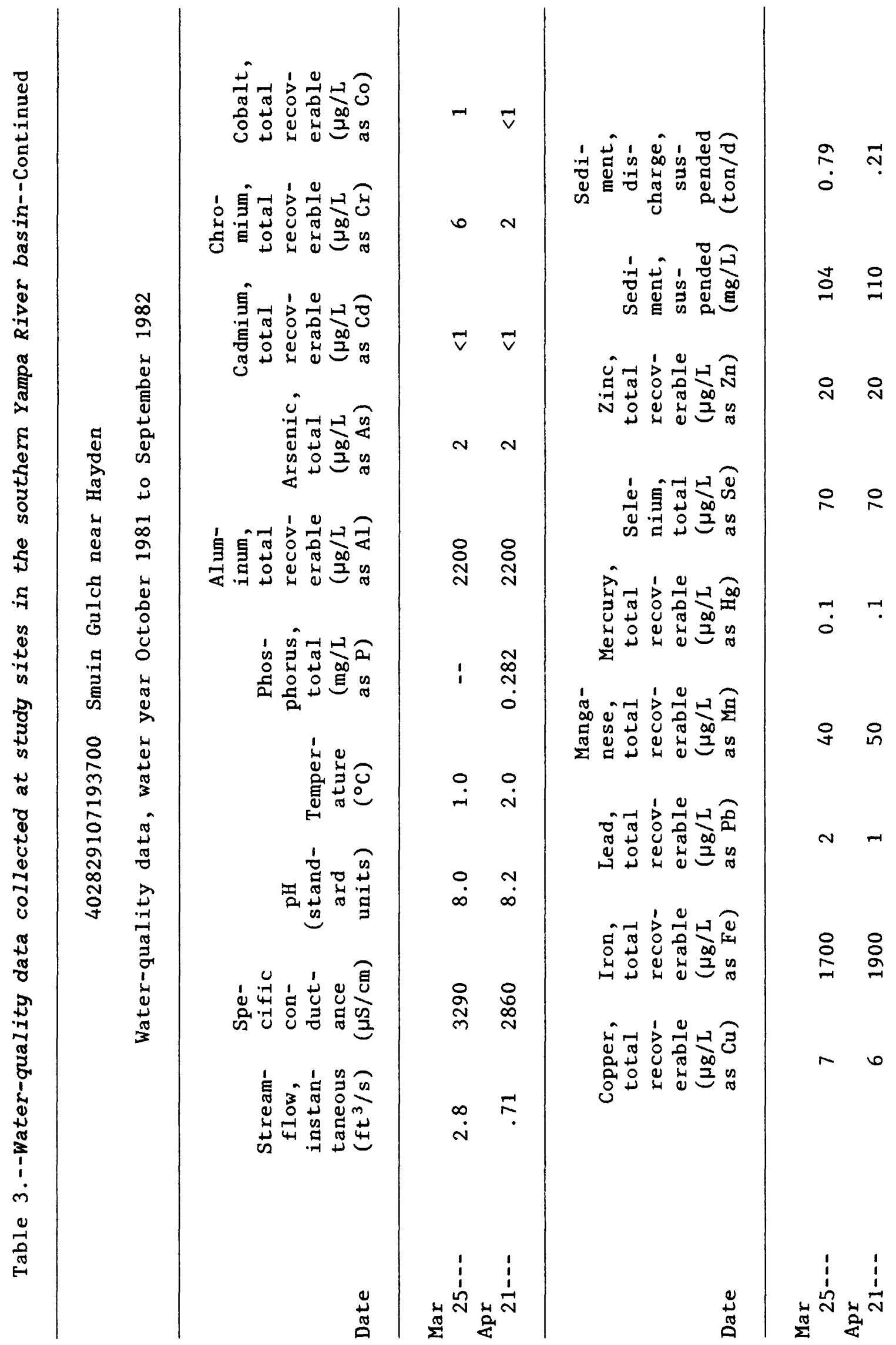




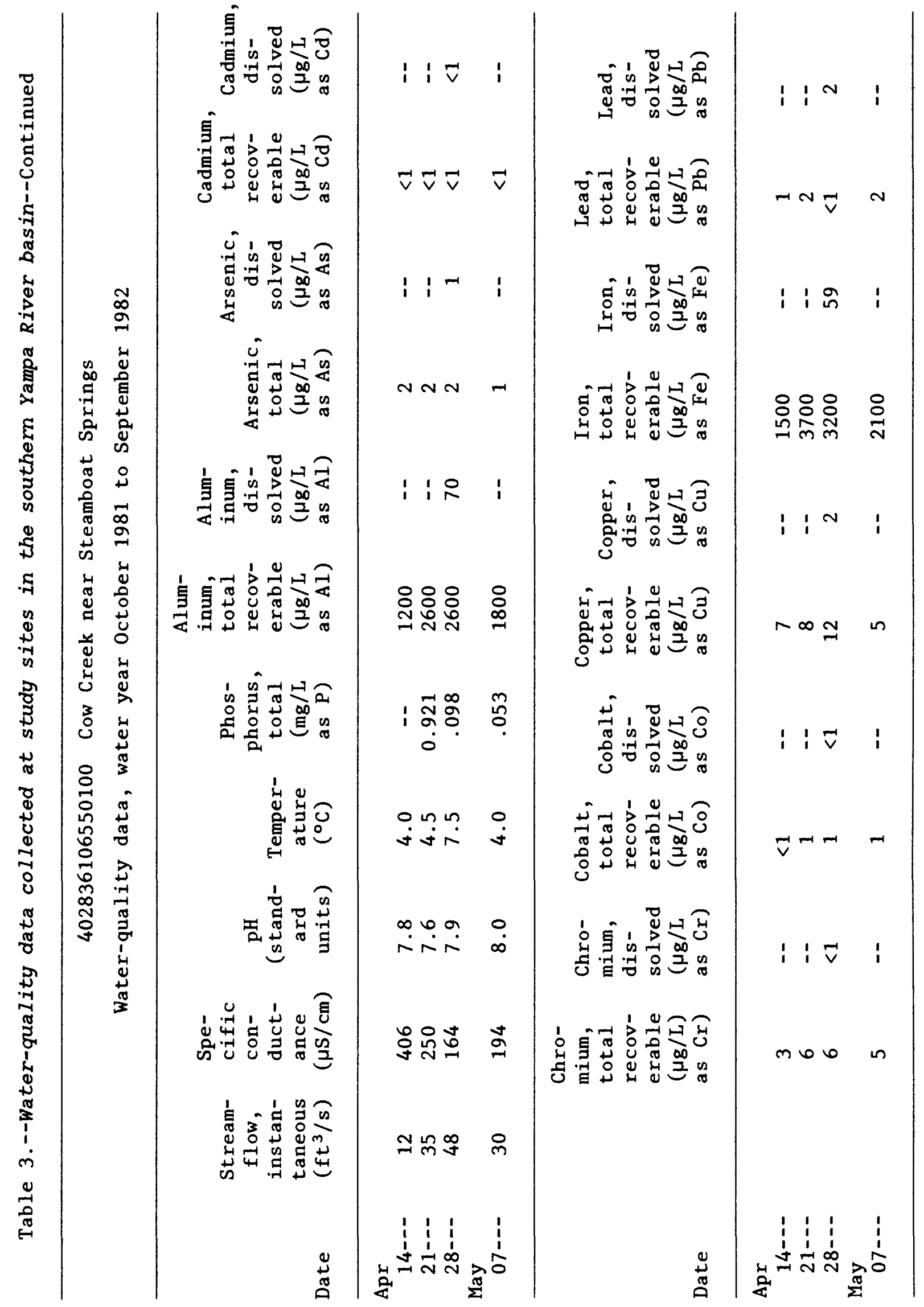




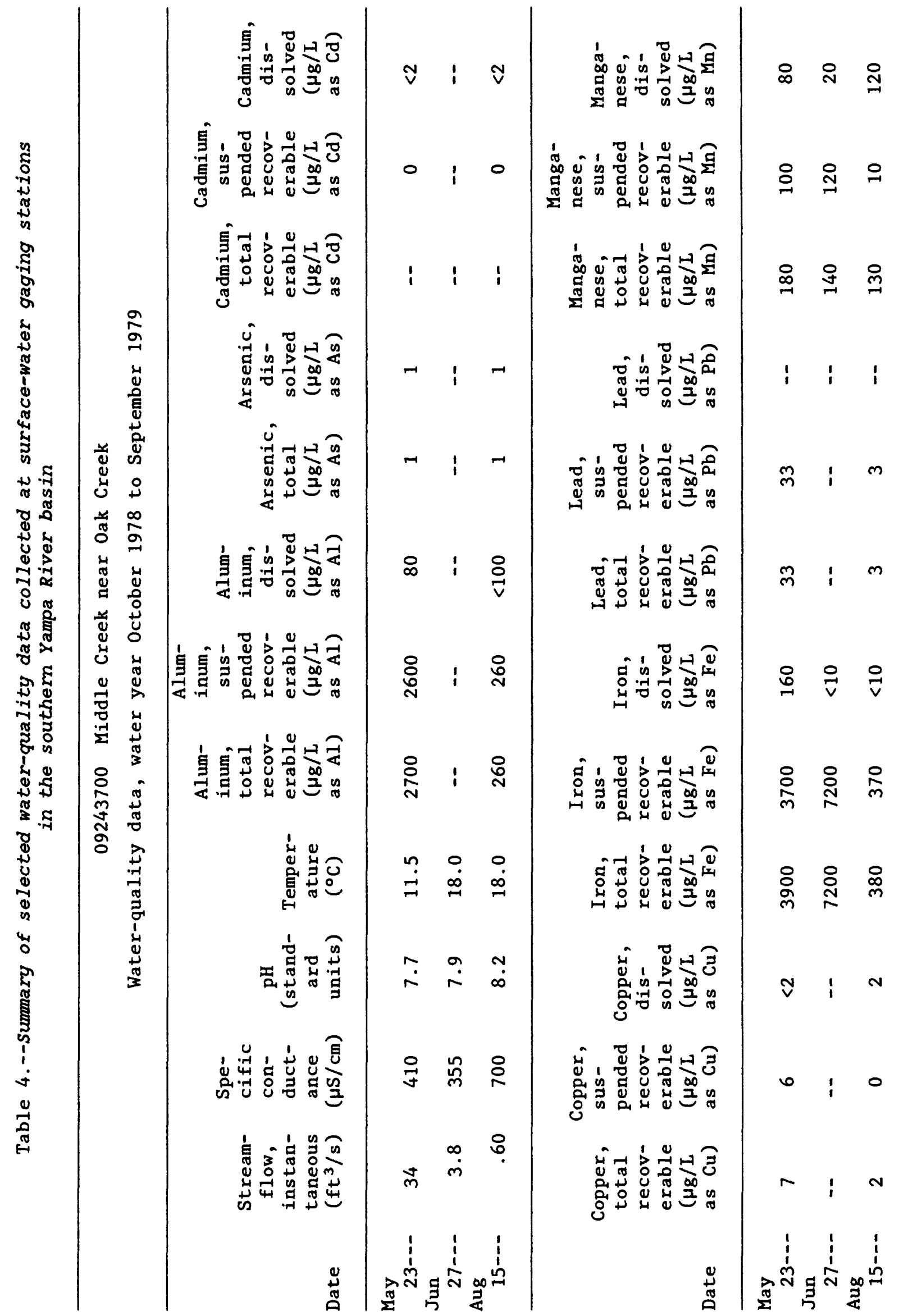




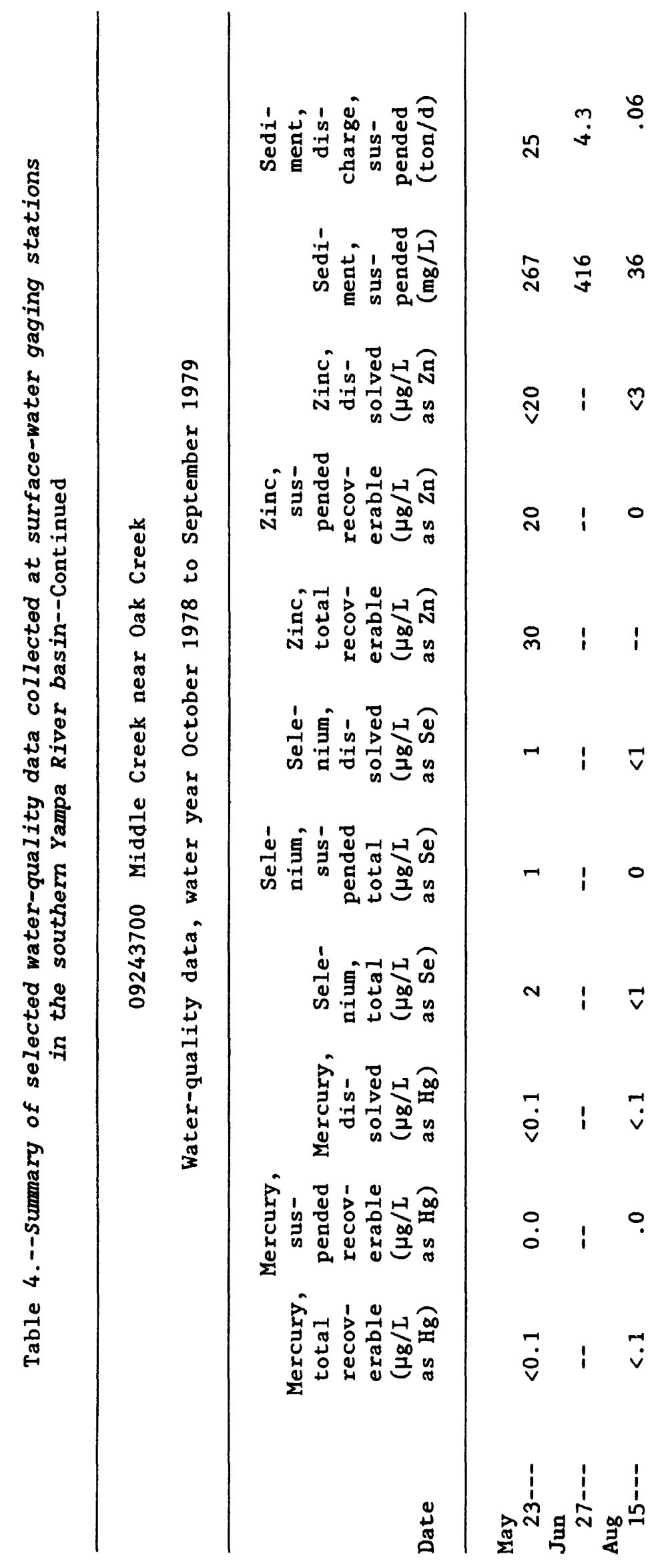




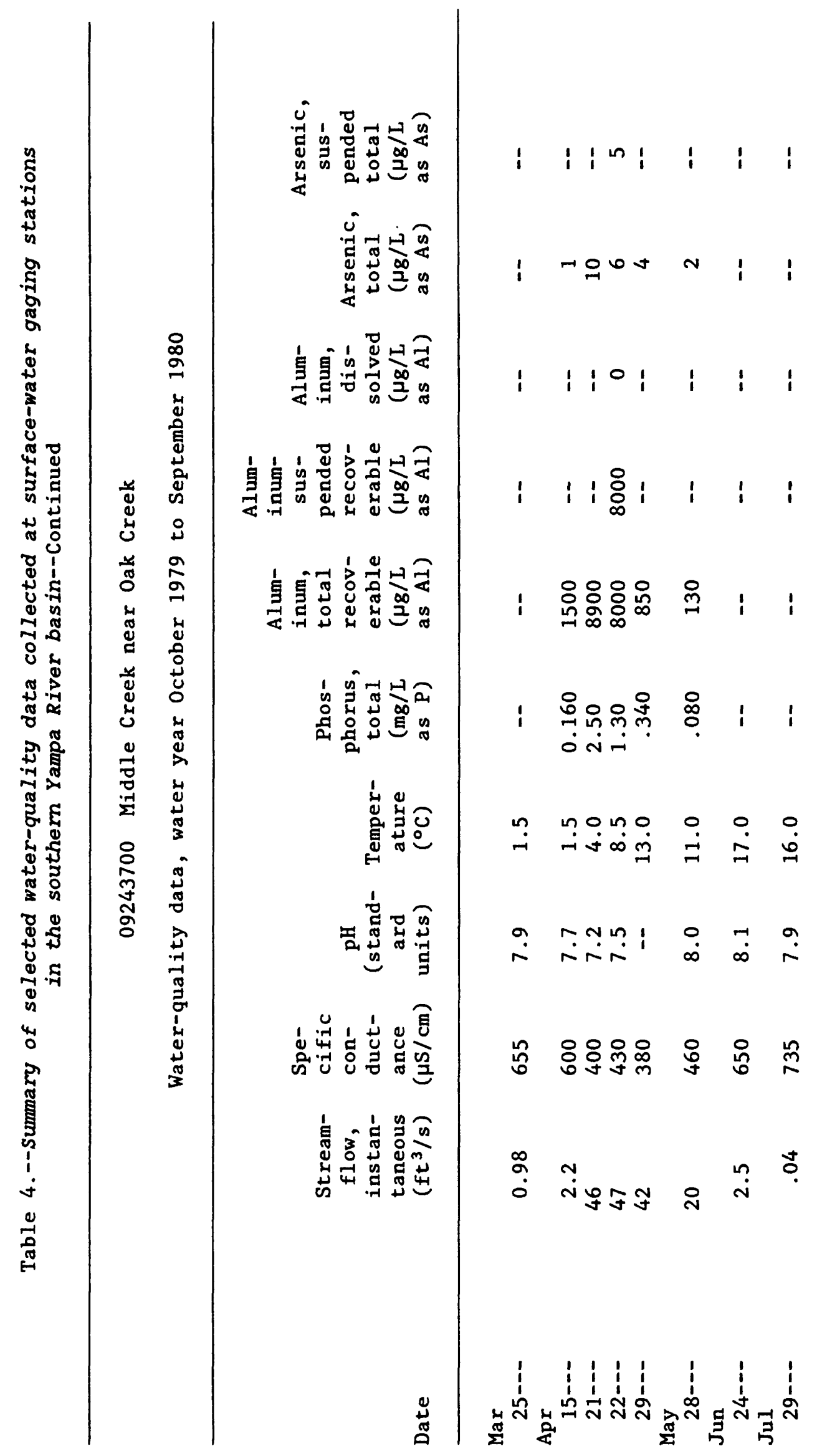




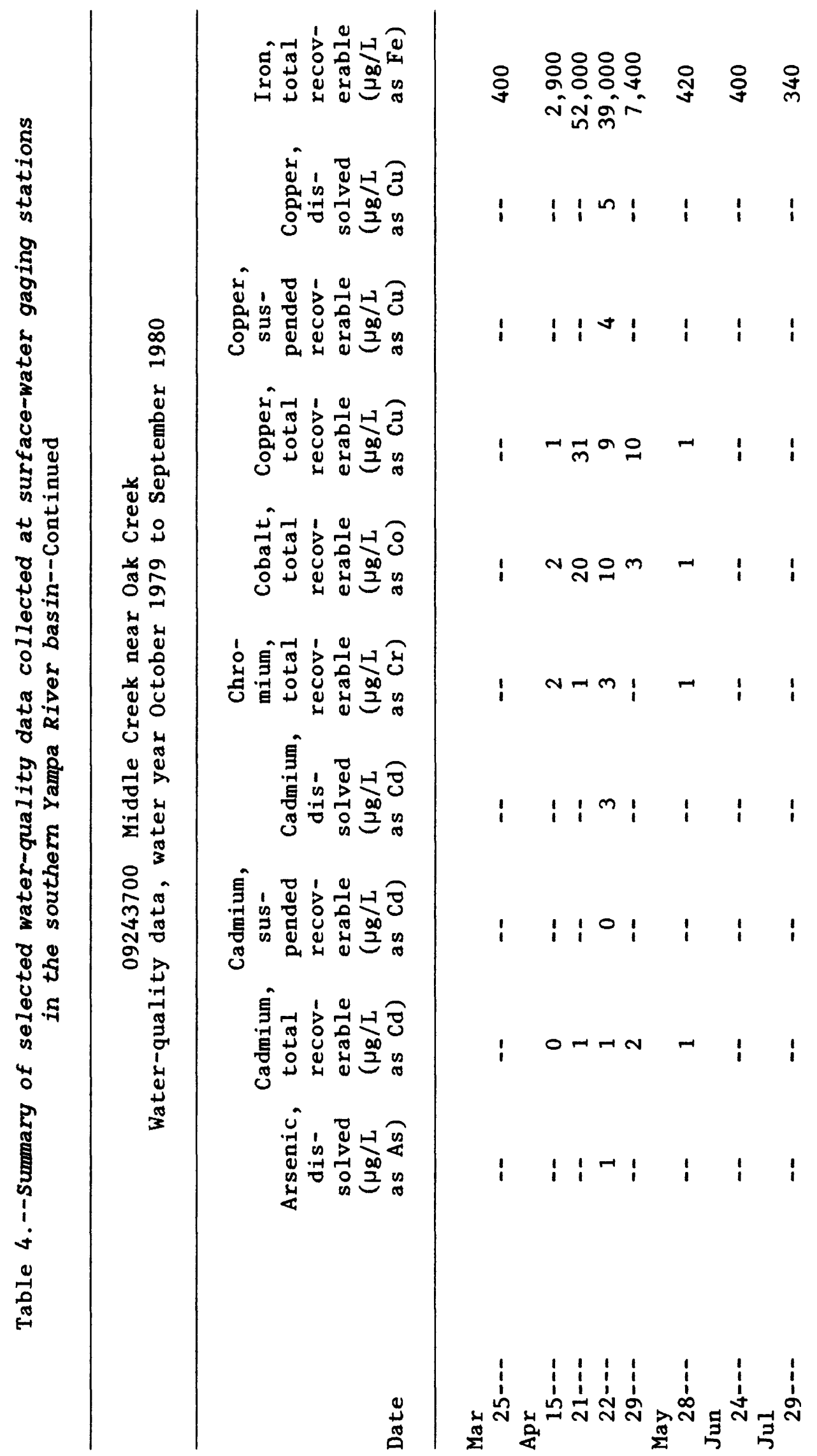




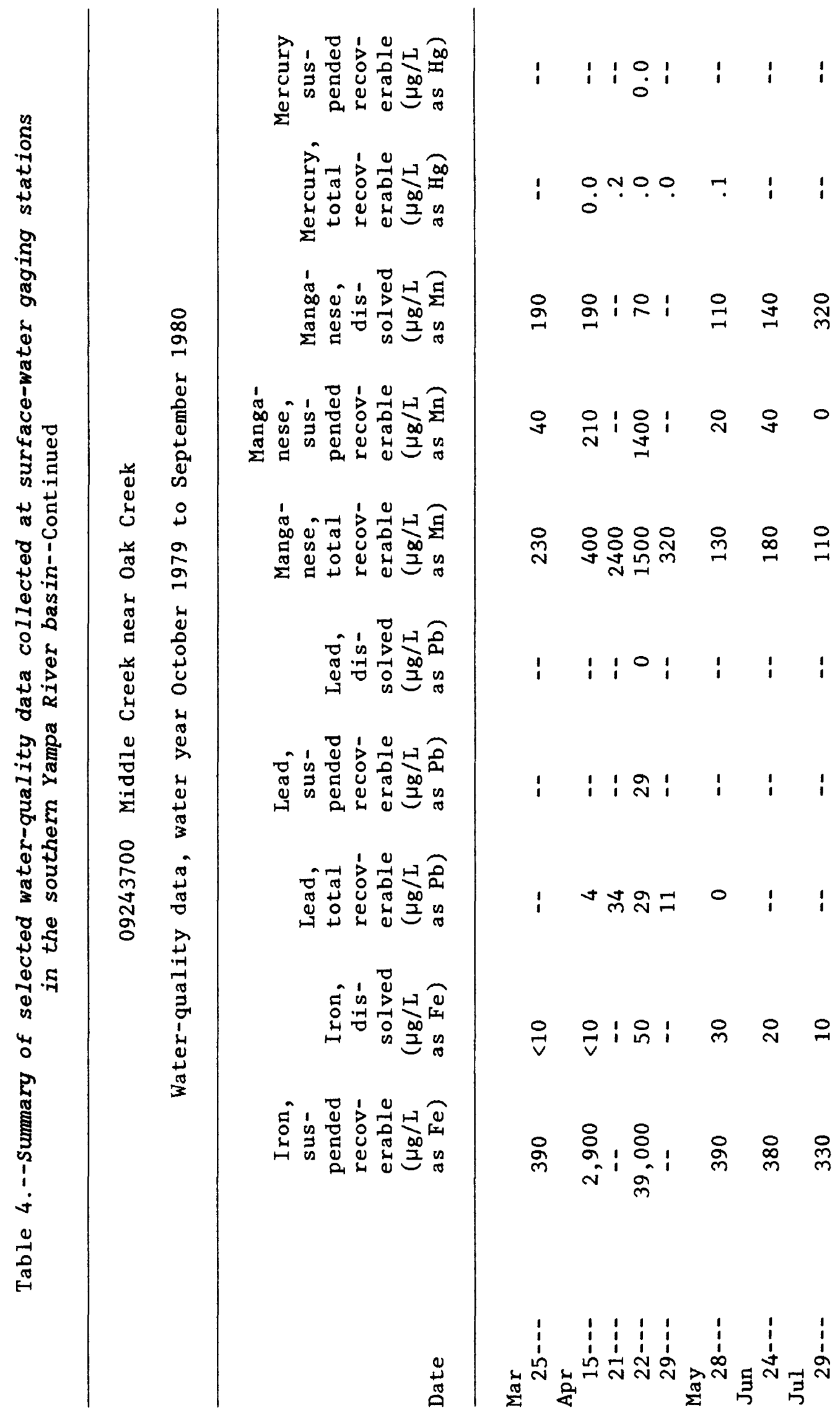




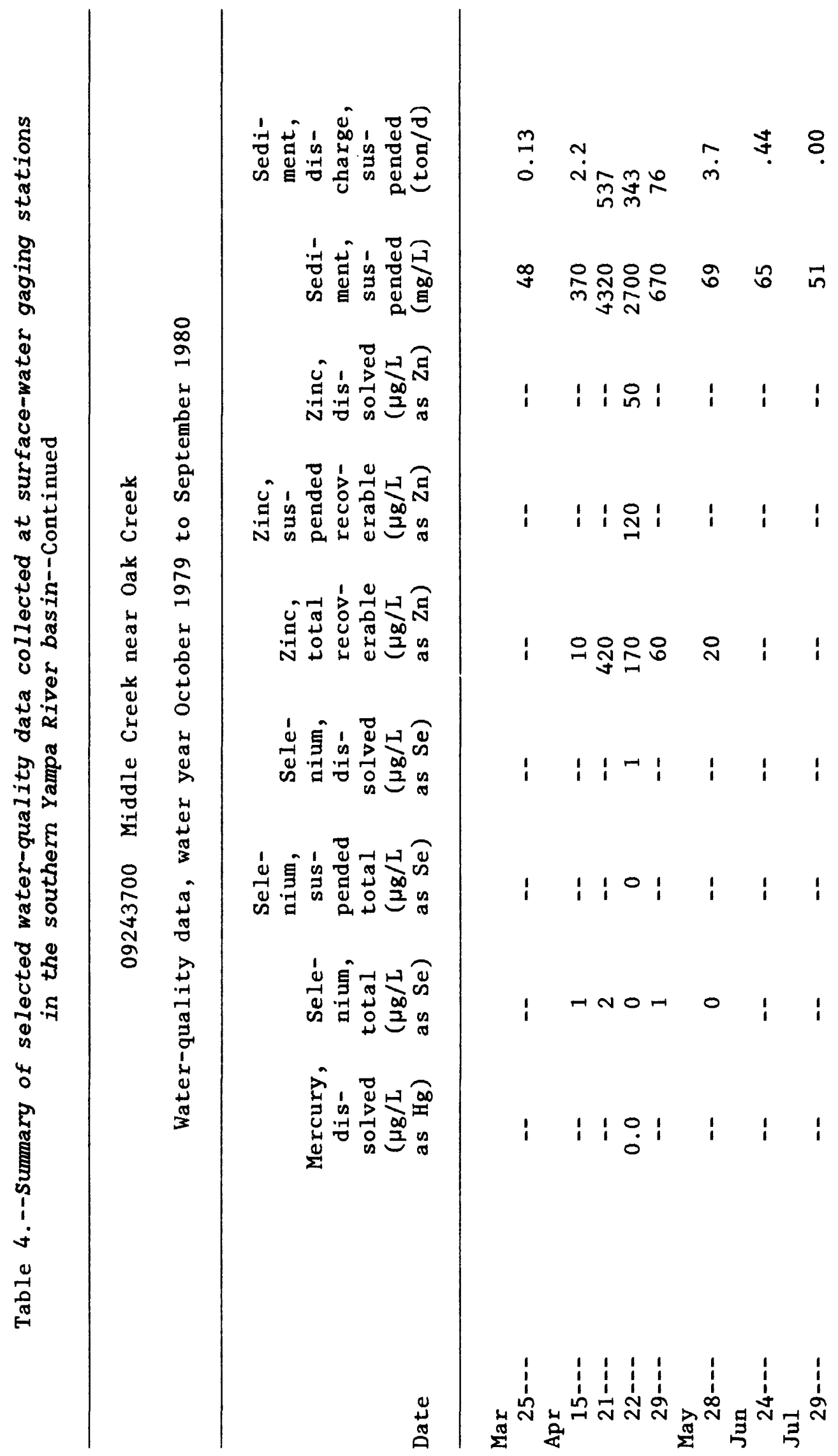




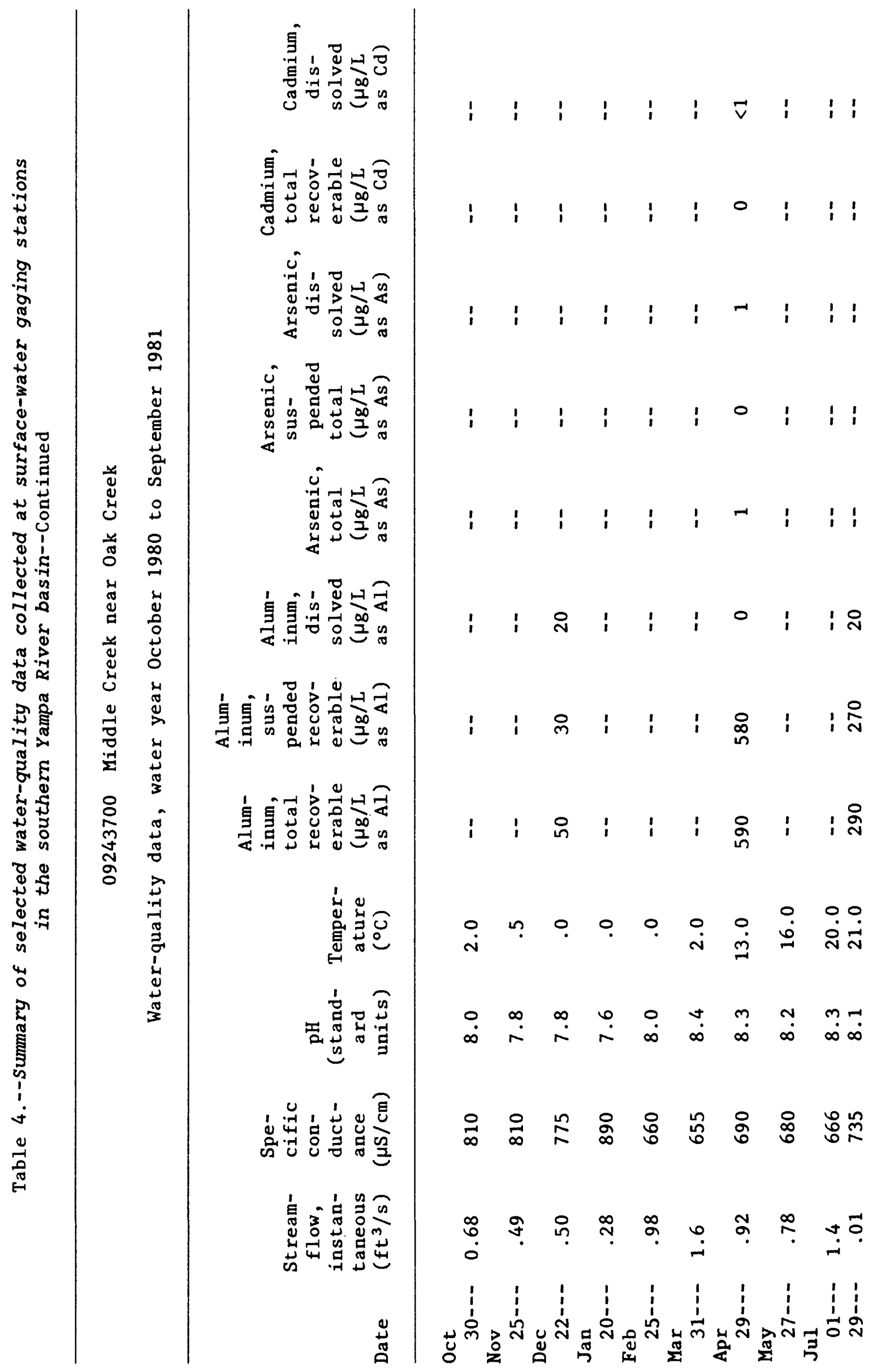




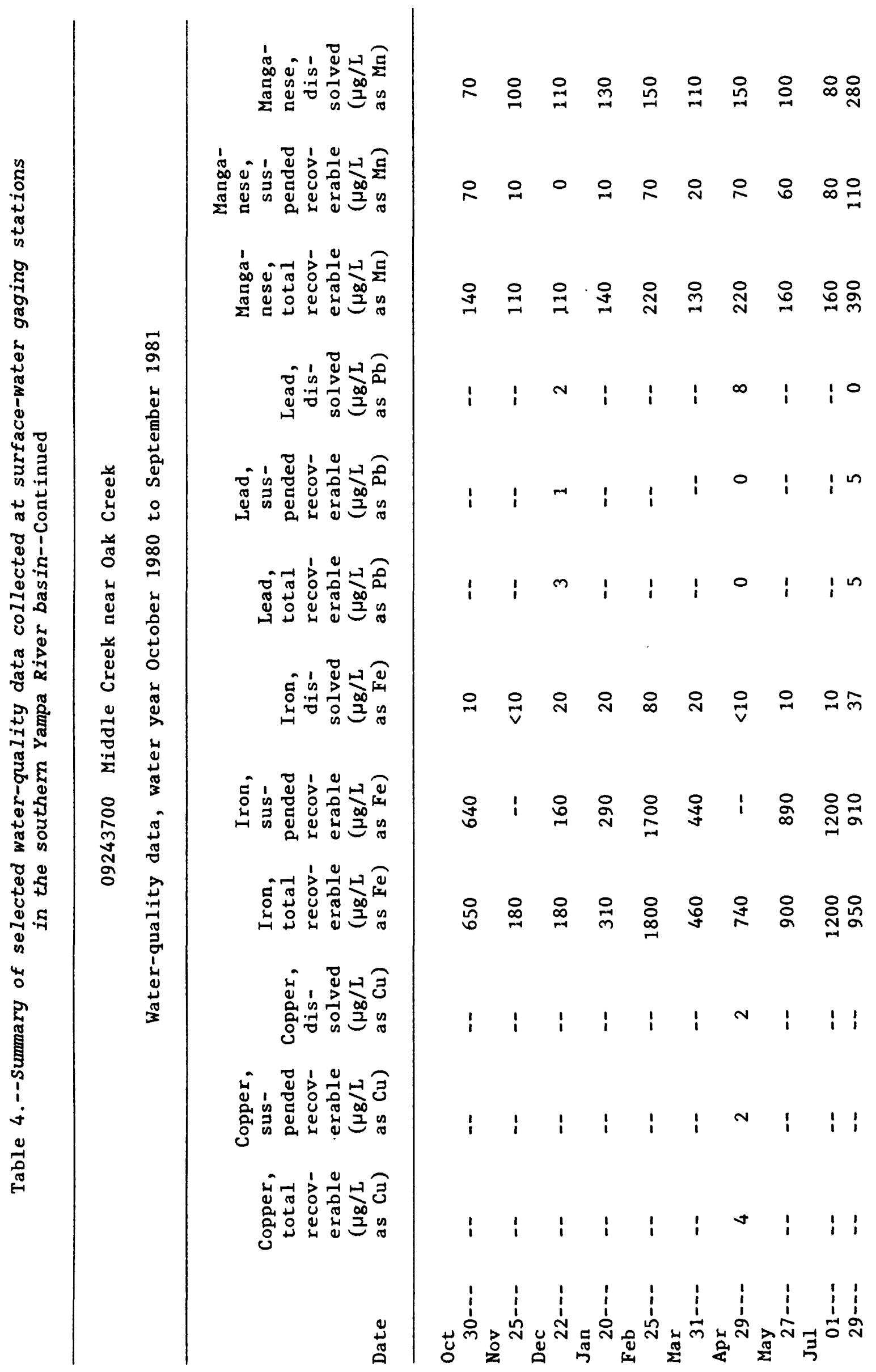




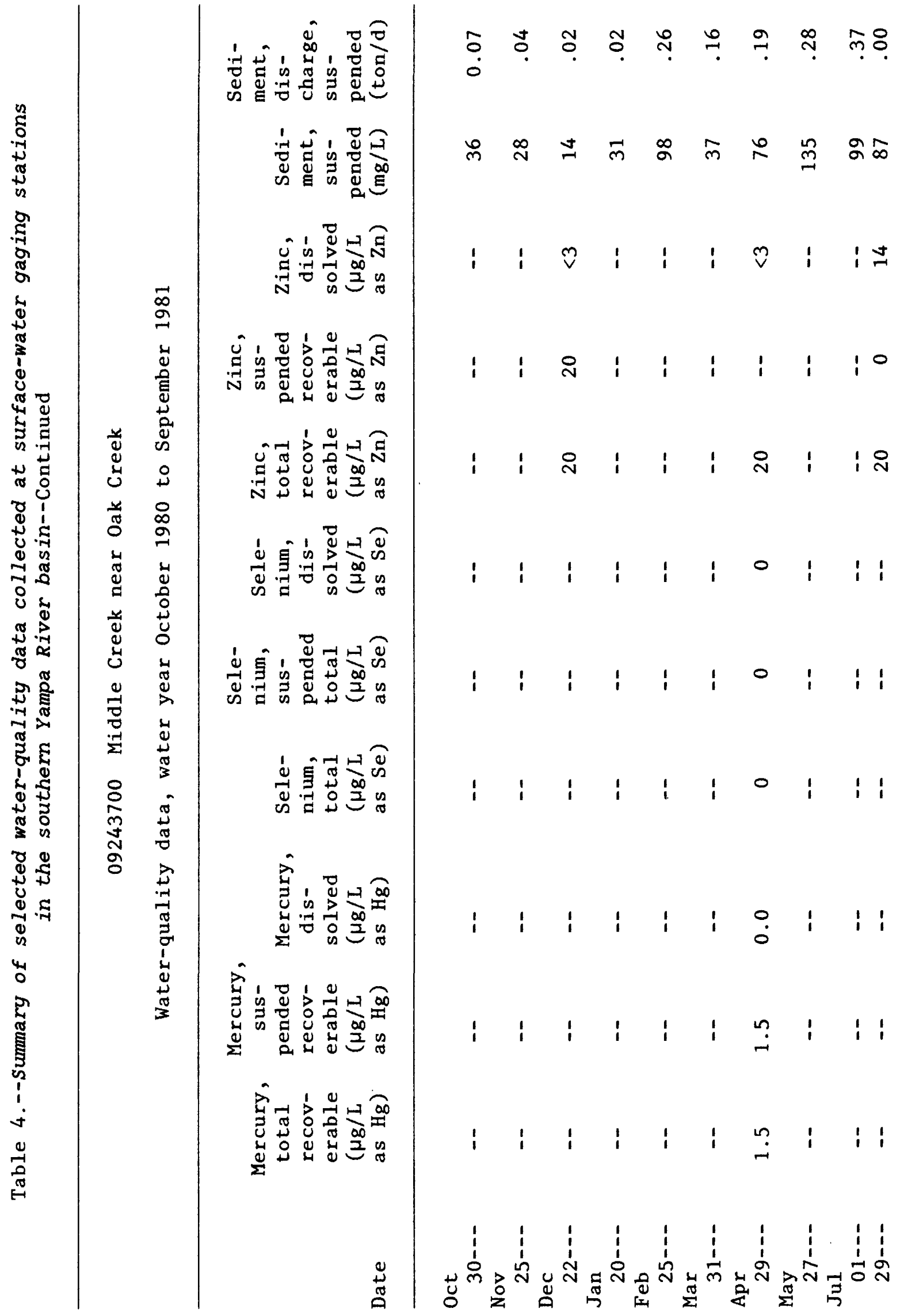




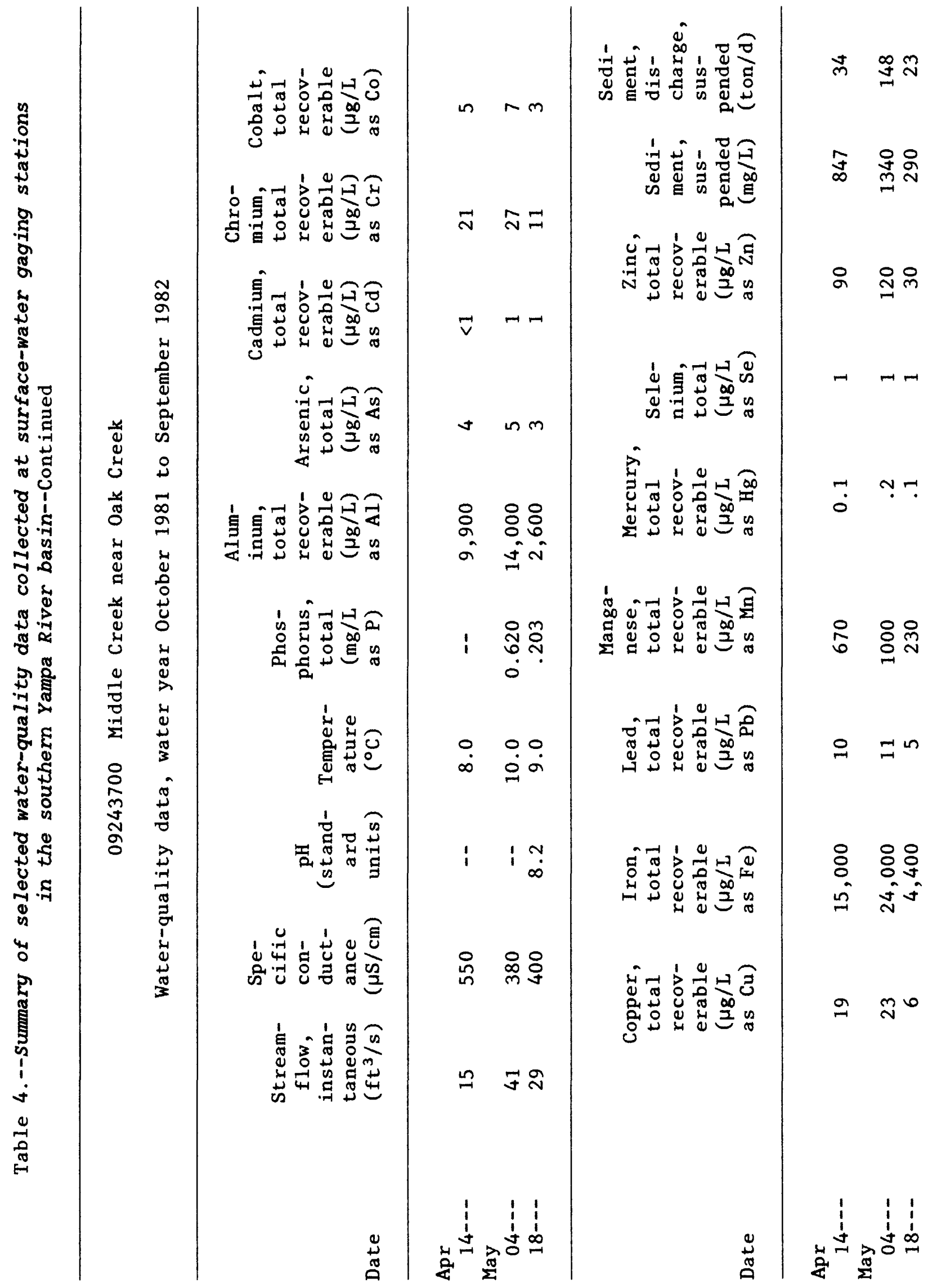




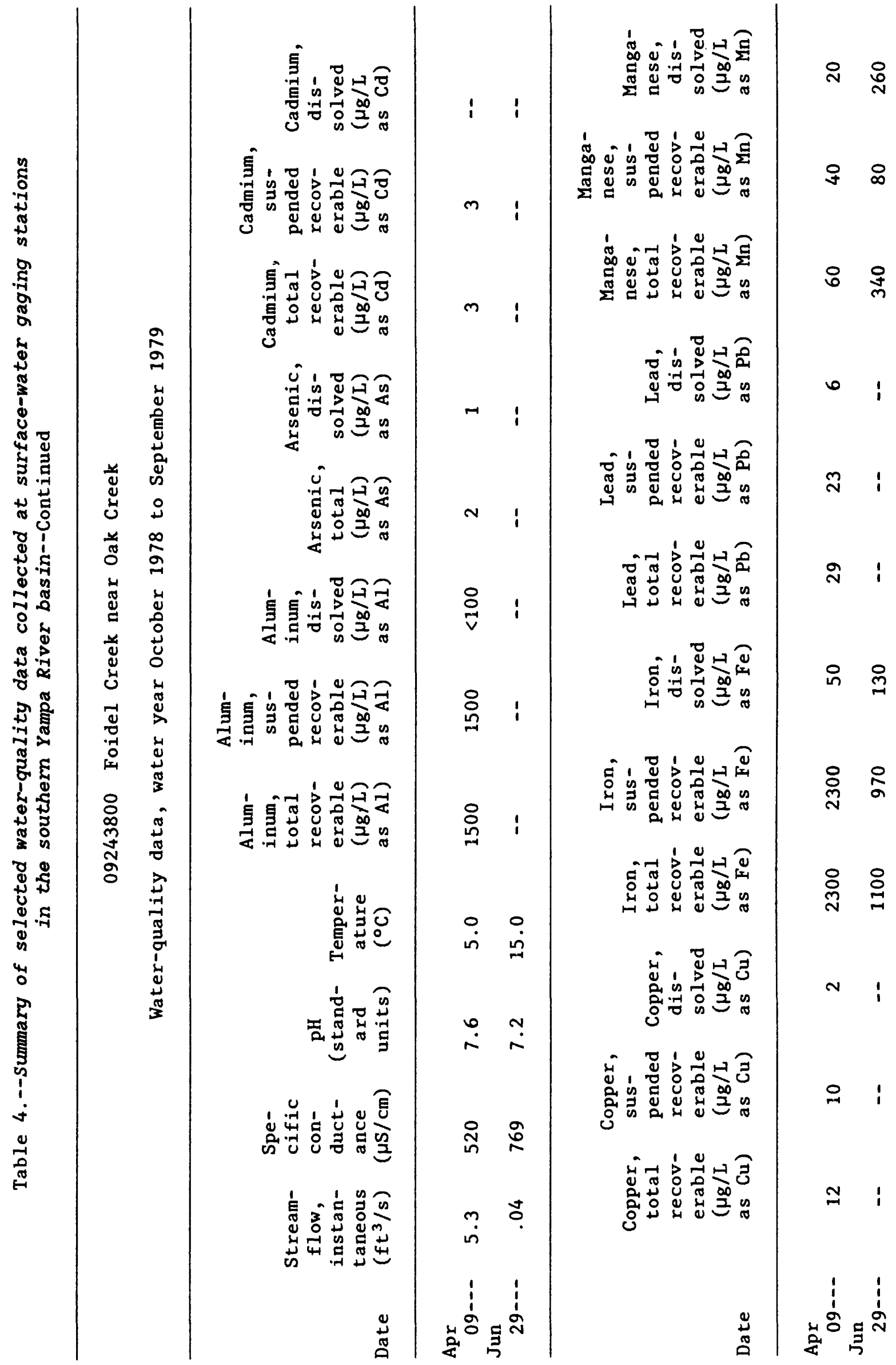




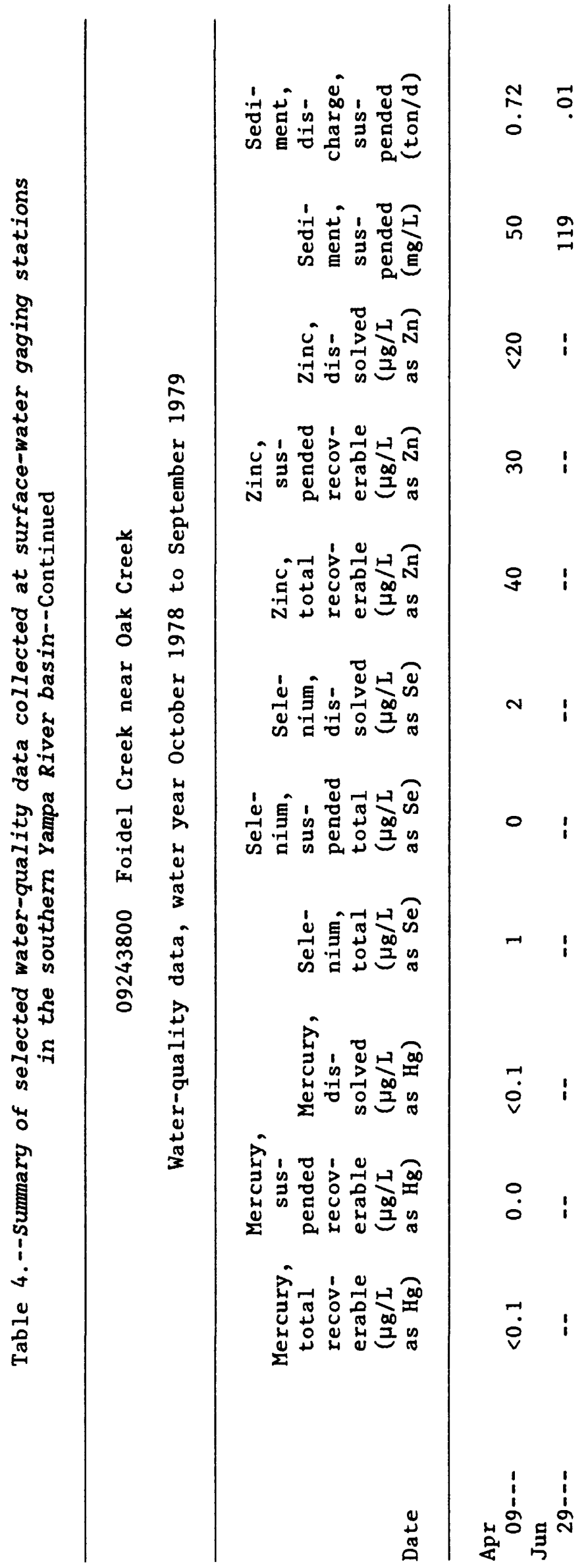




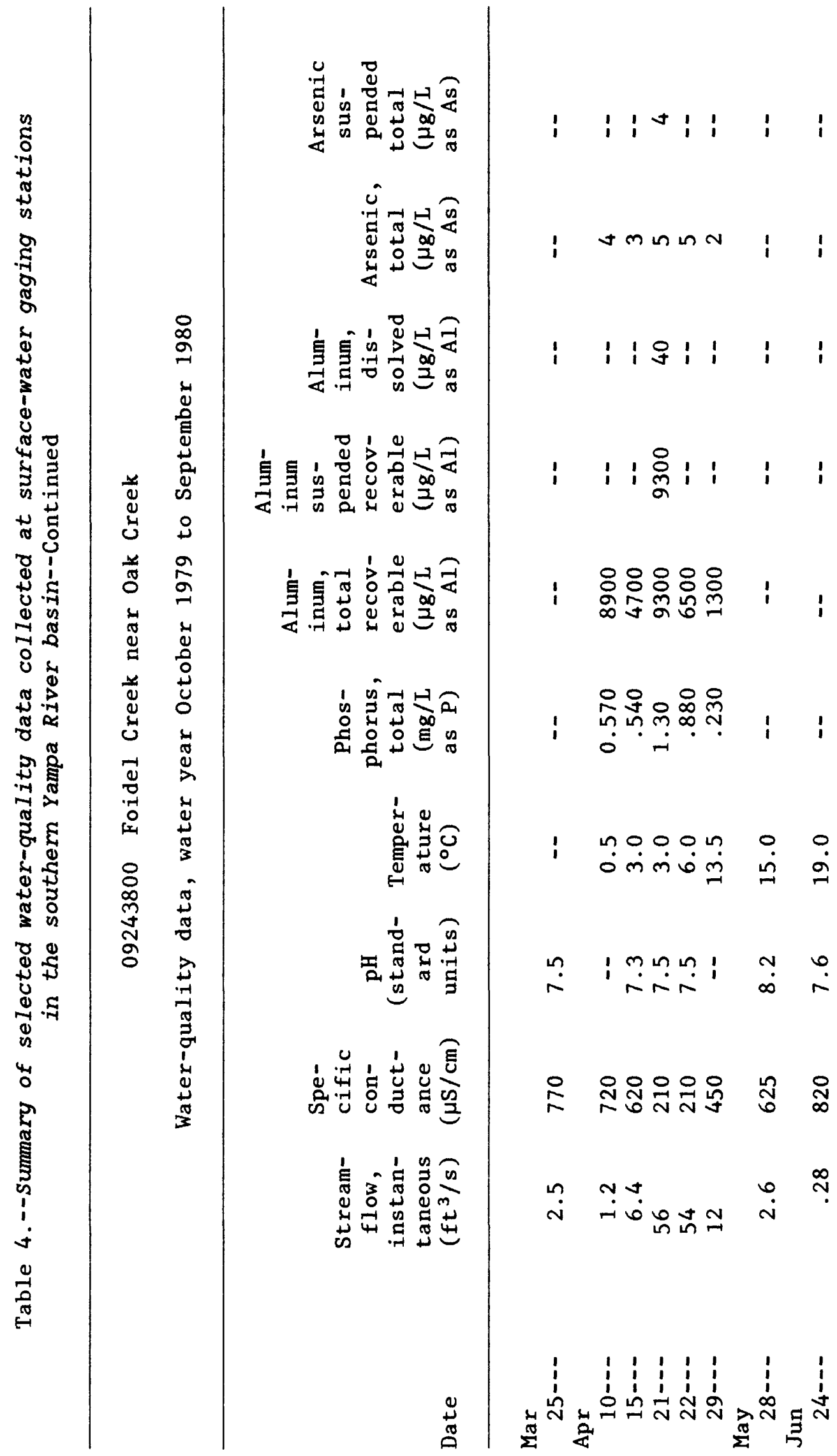




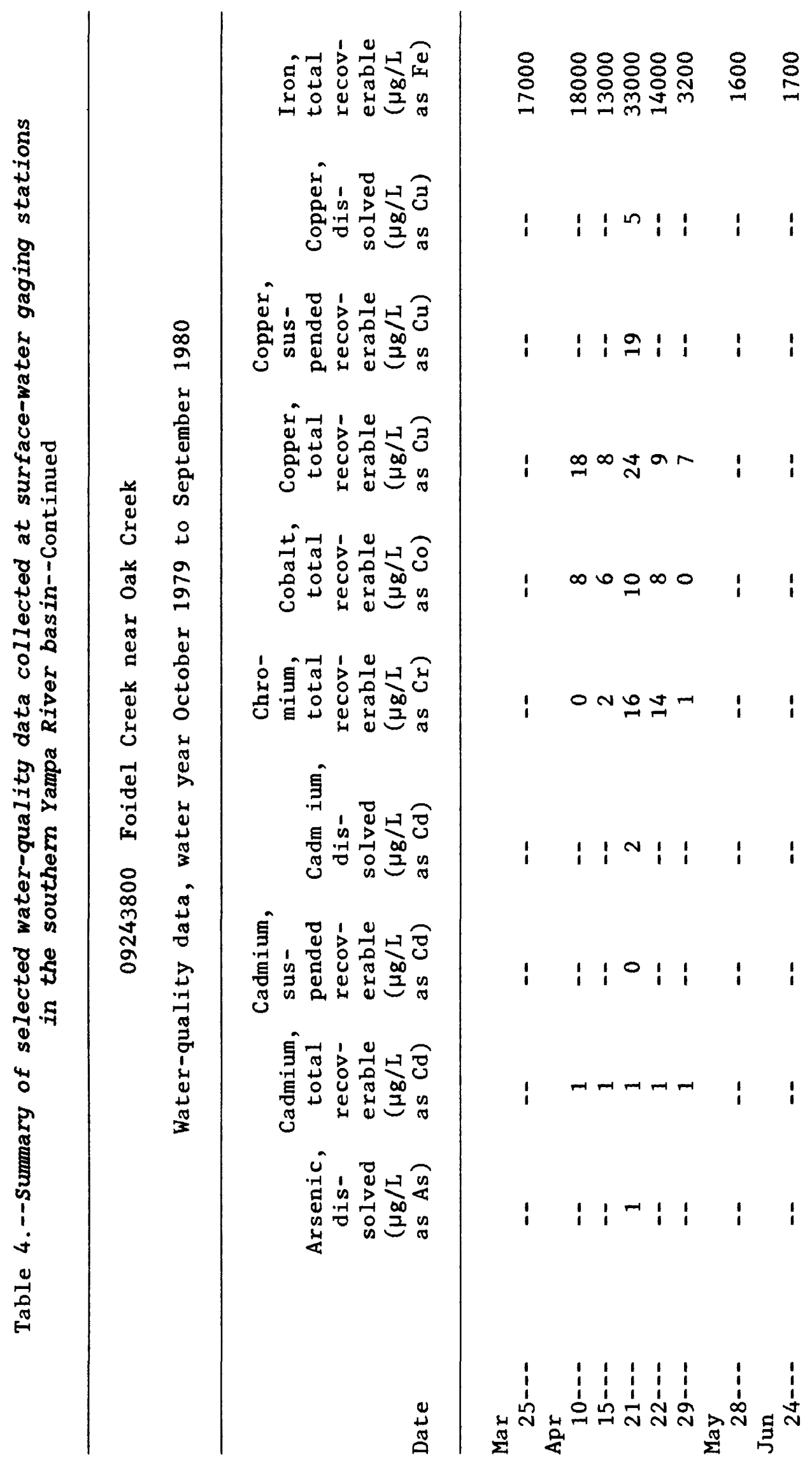




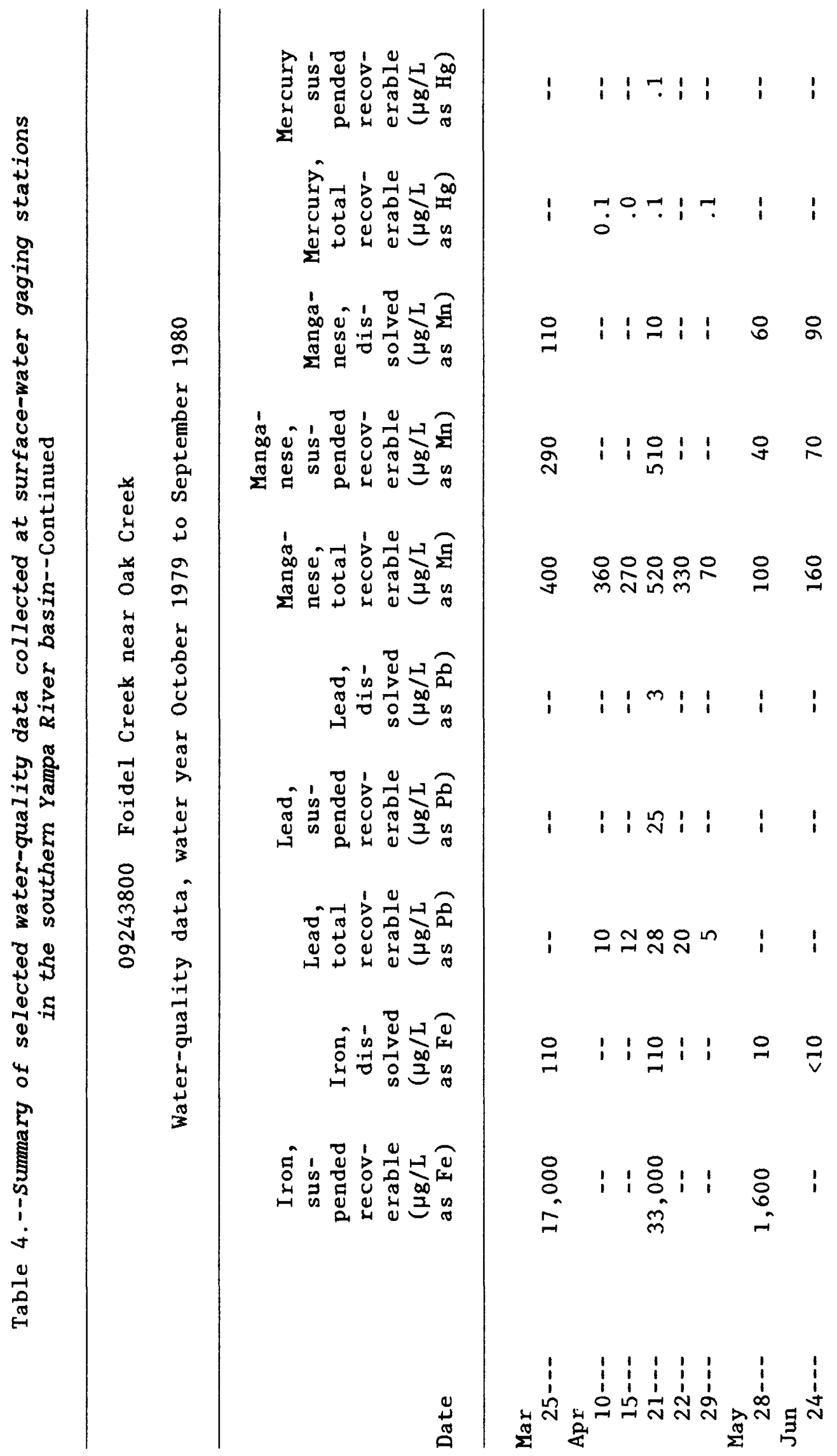




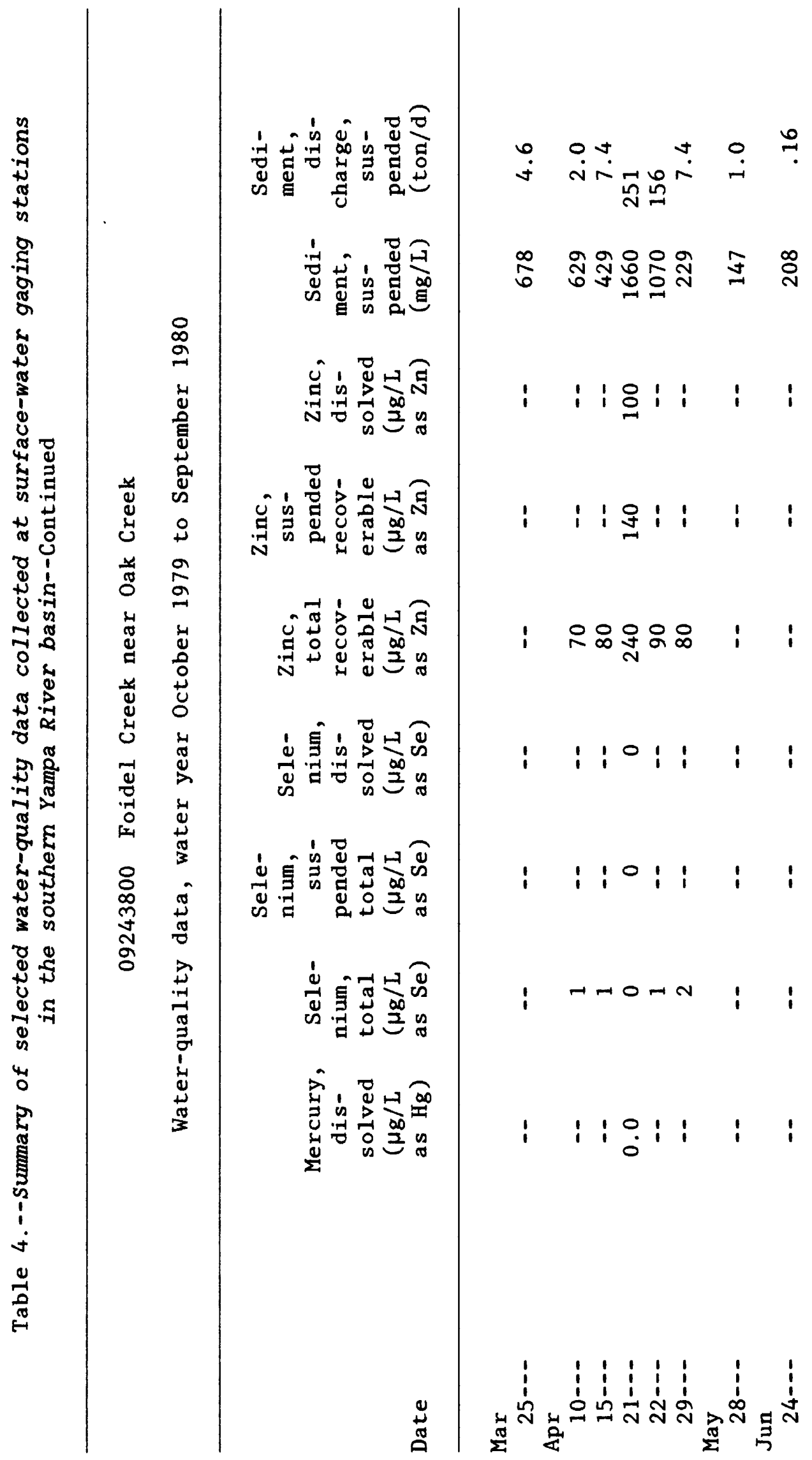




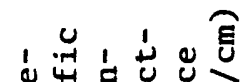
की

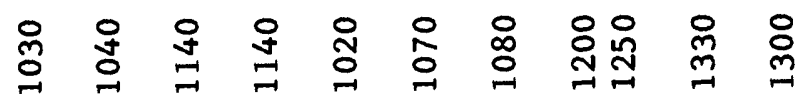

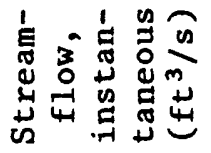

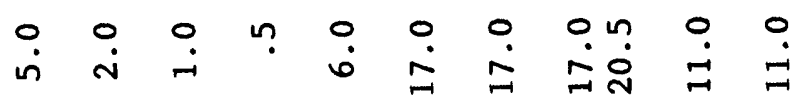

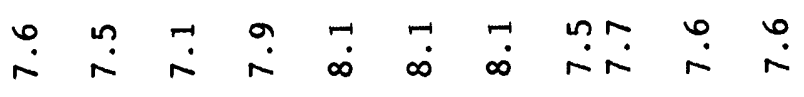




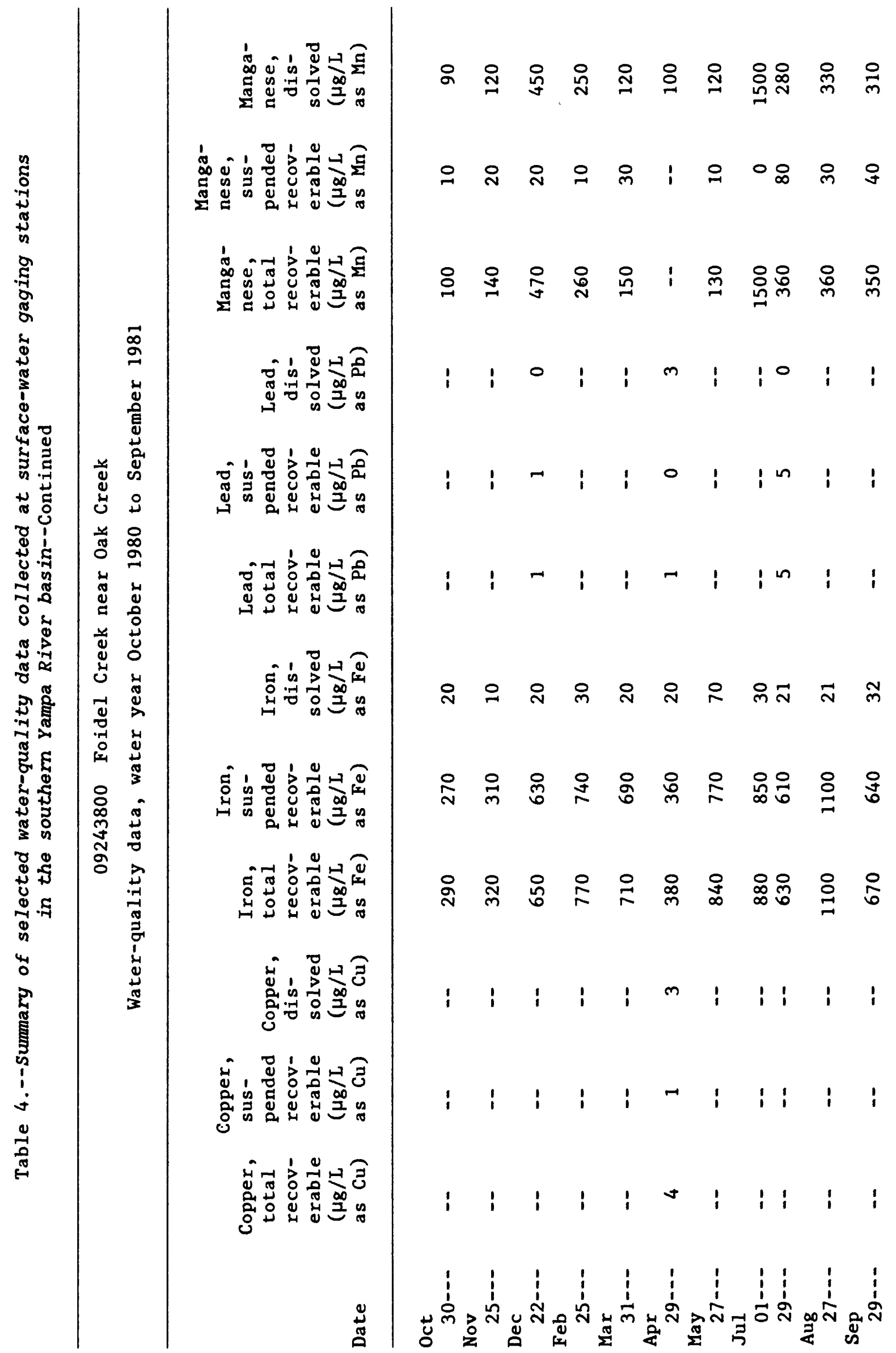




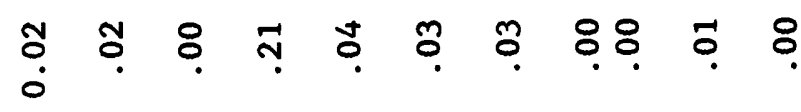

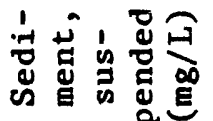
สี กี บ, จำ

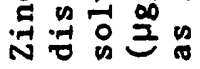




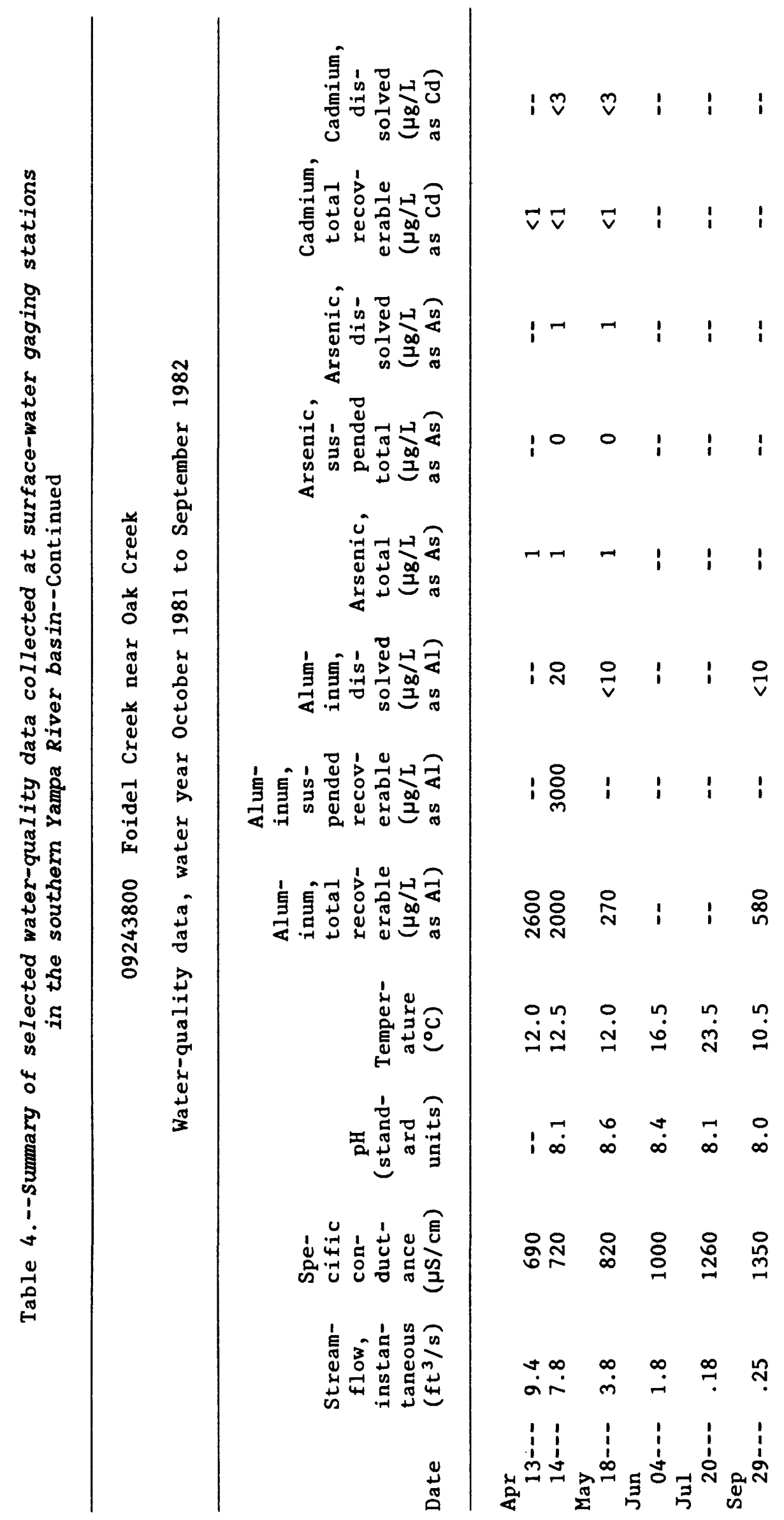




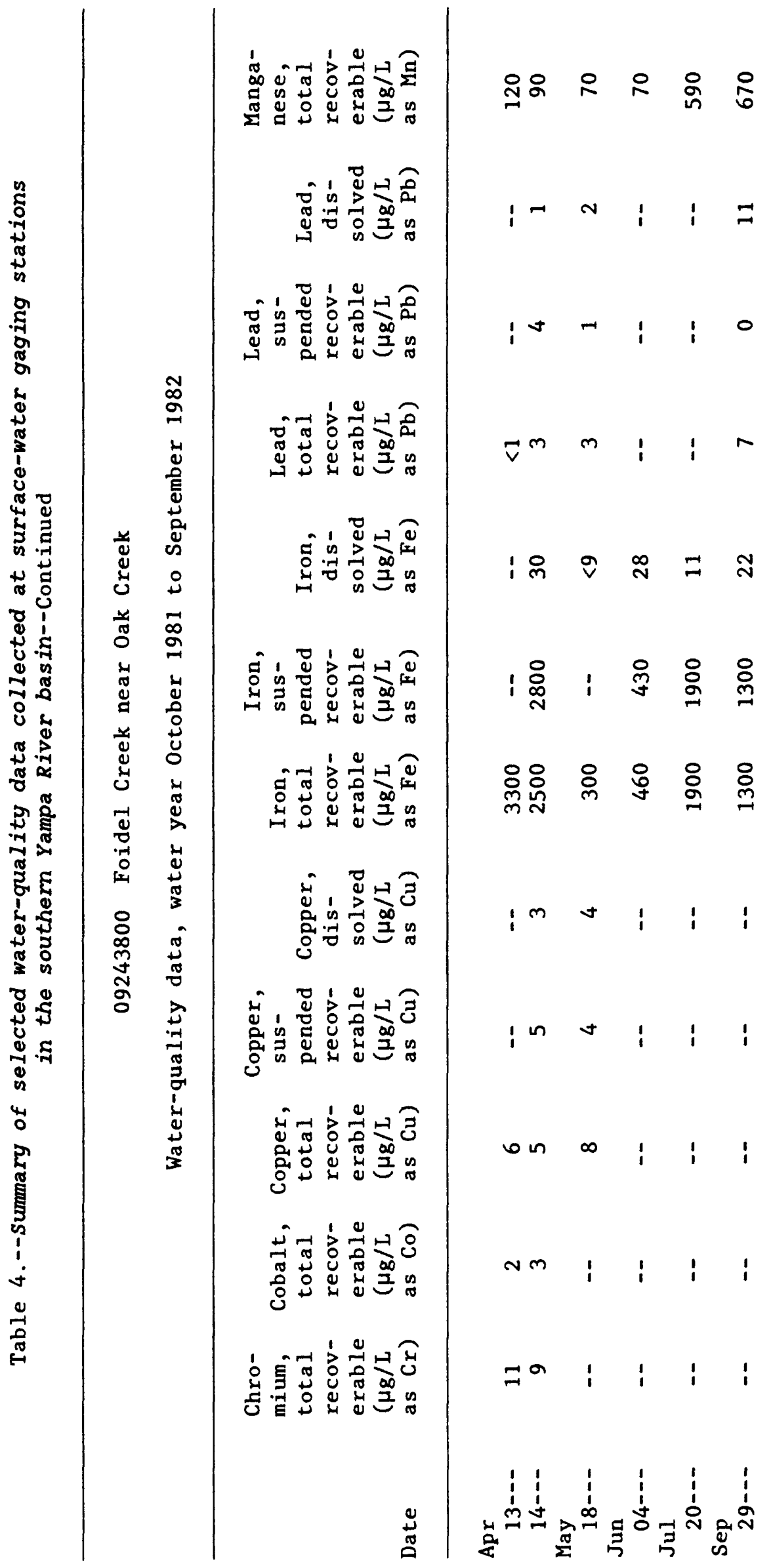




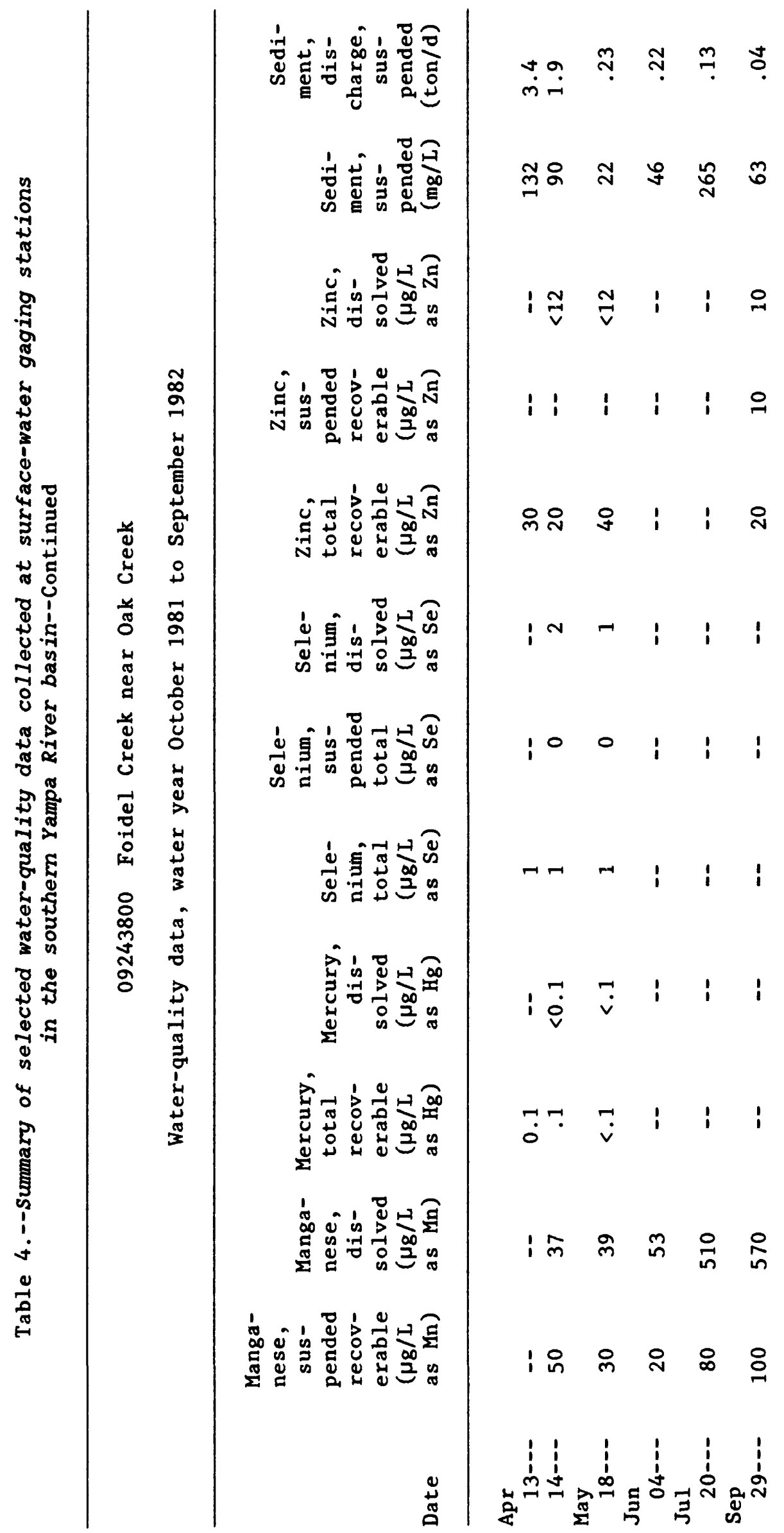




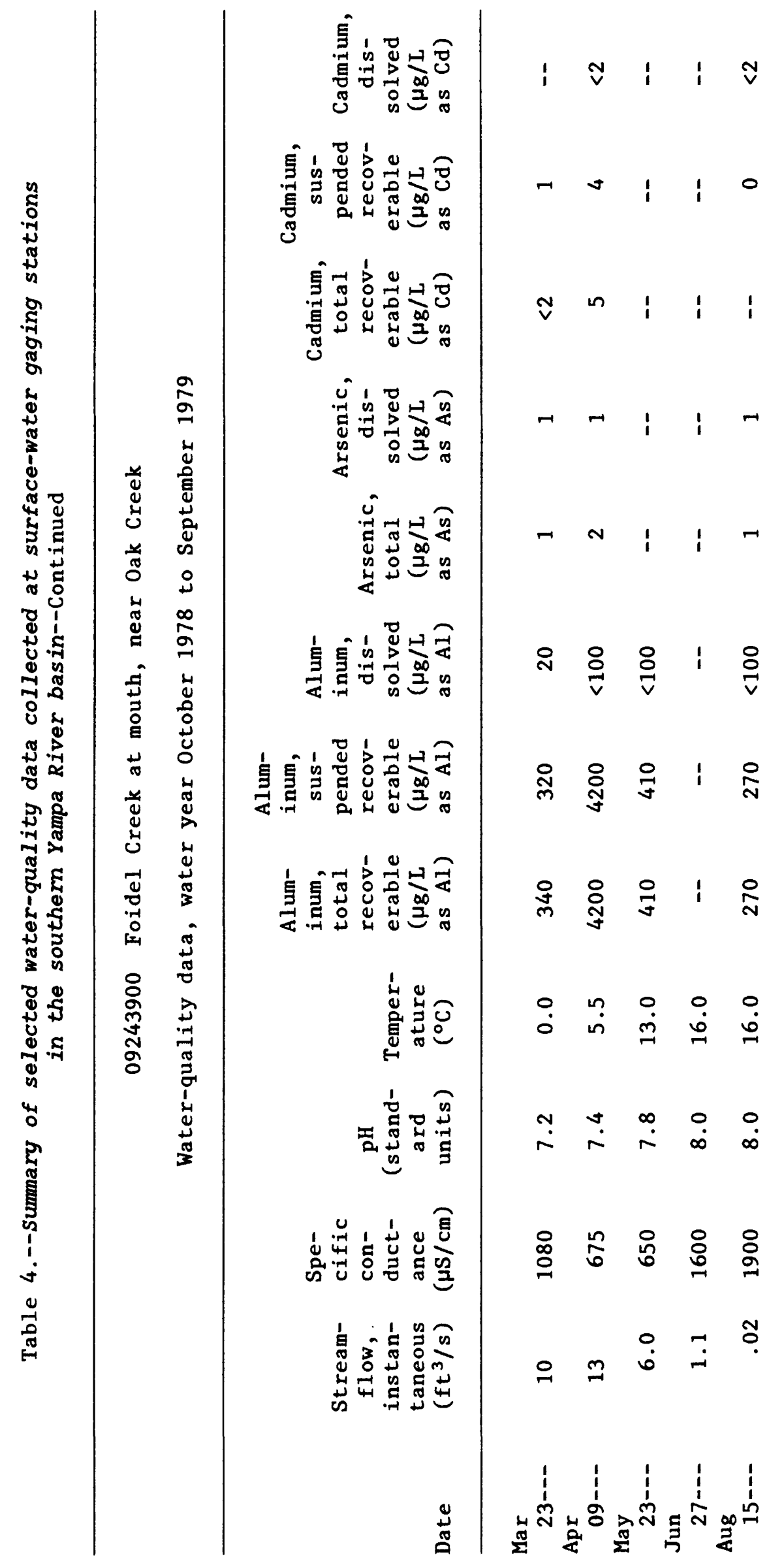




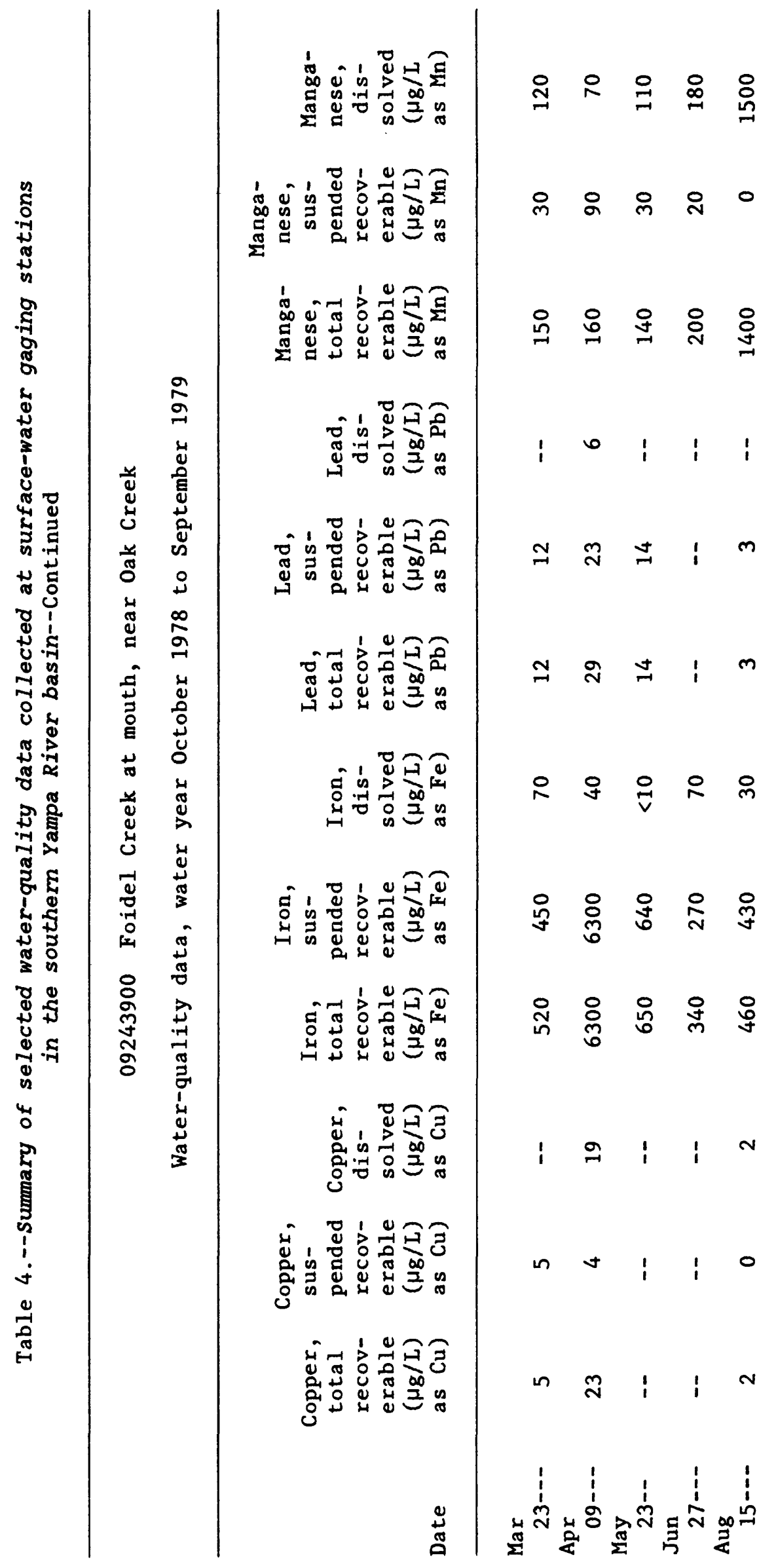




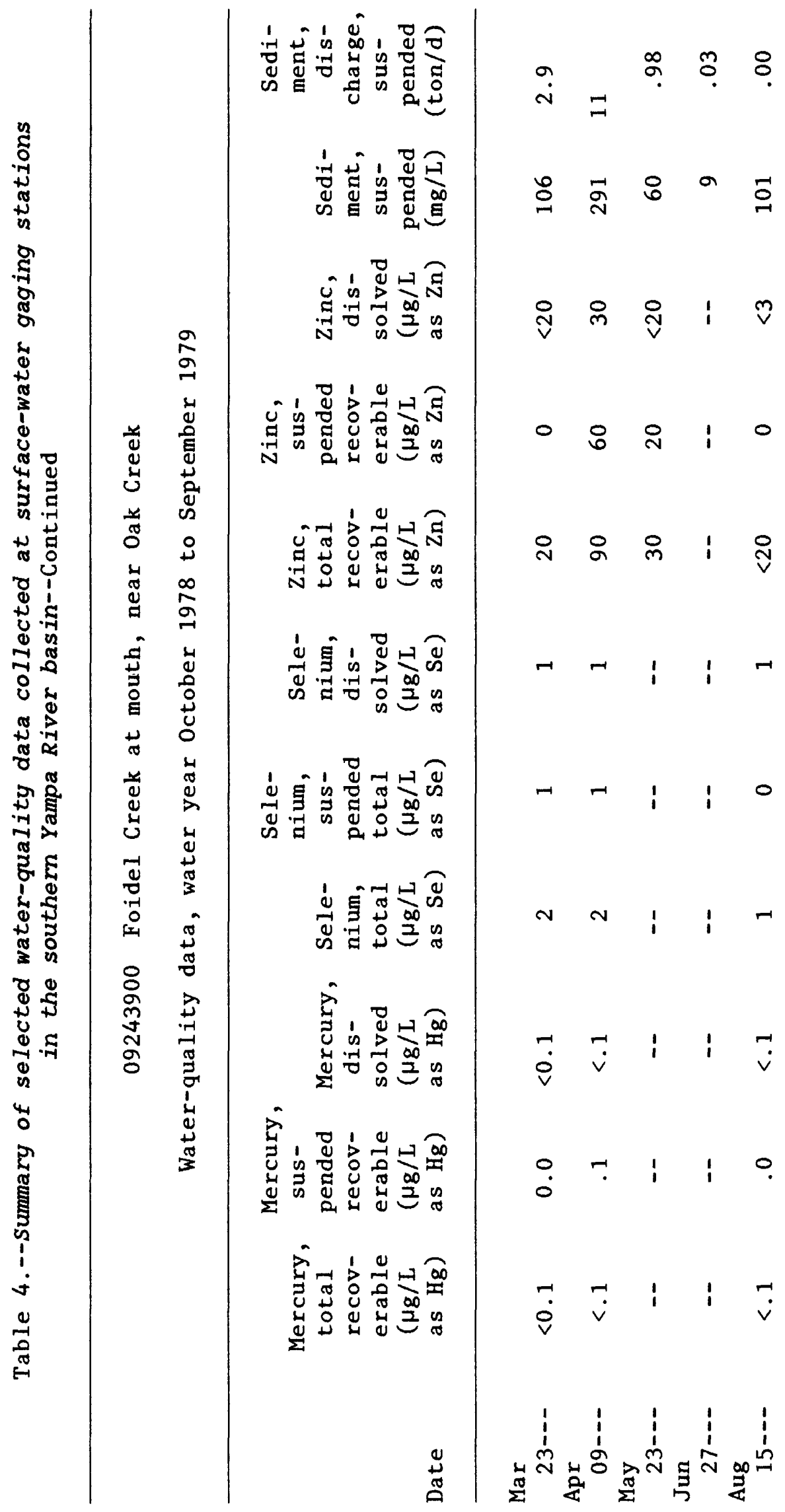




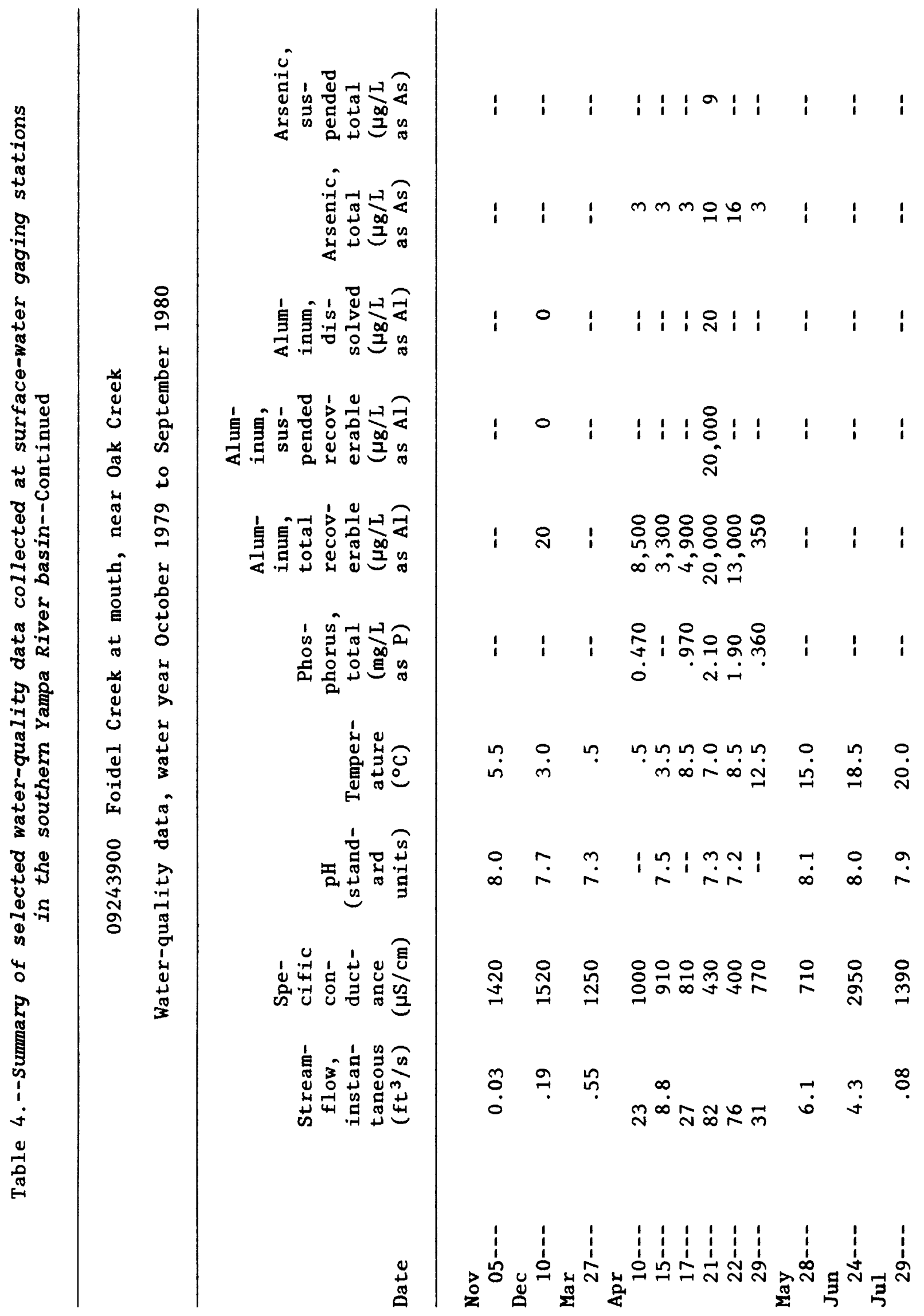




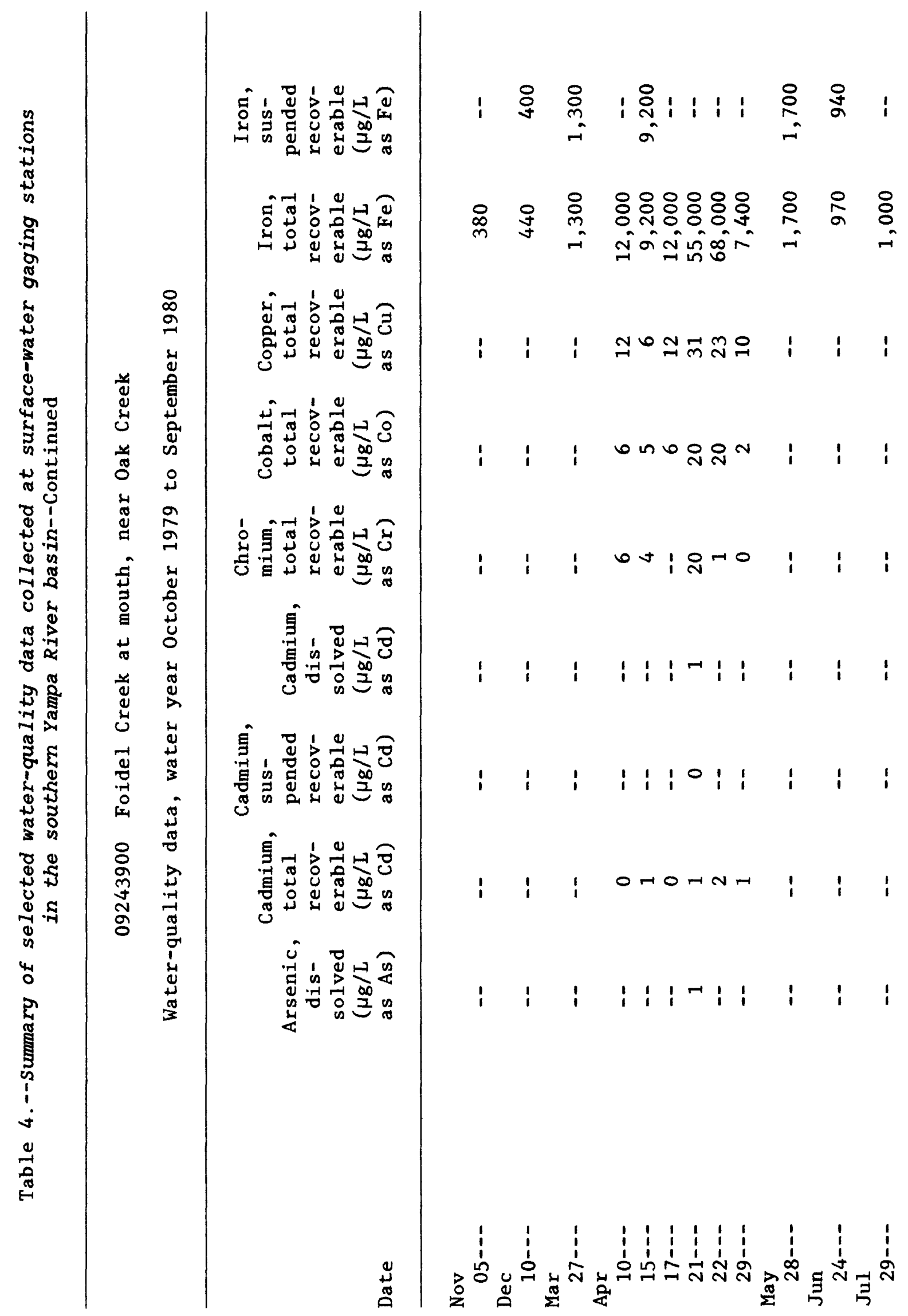




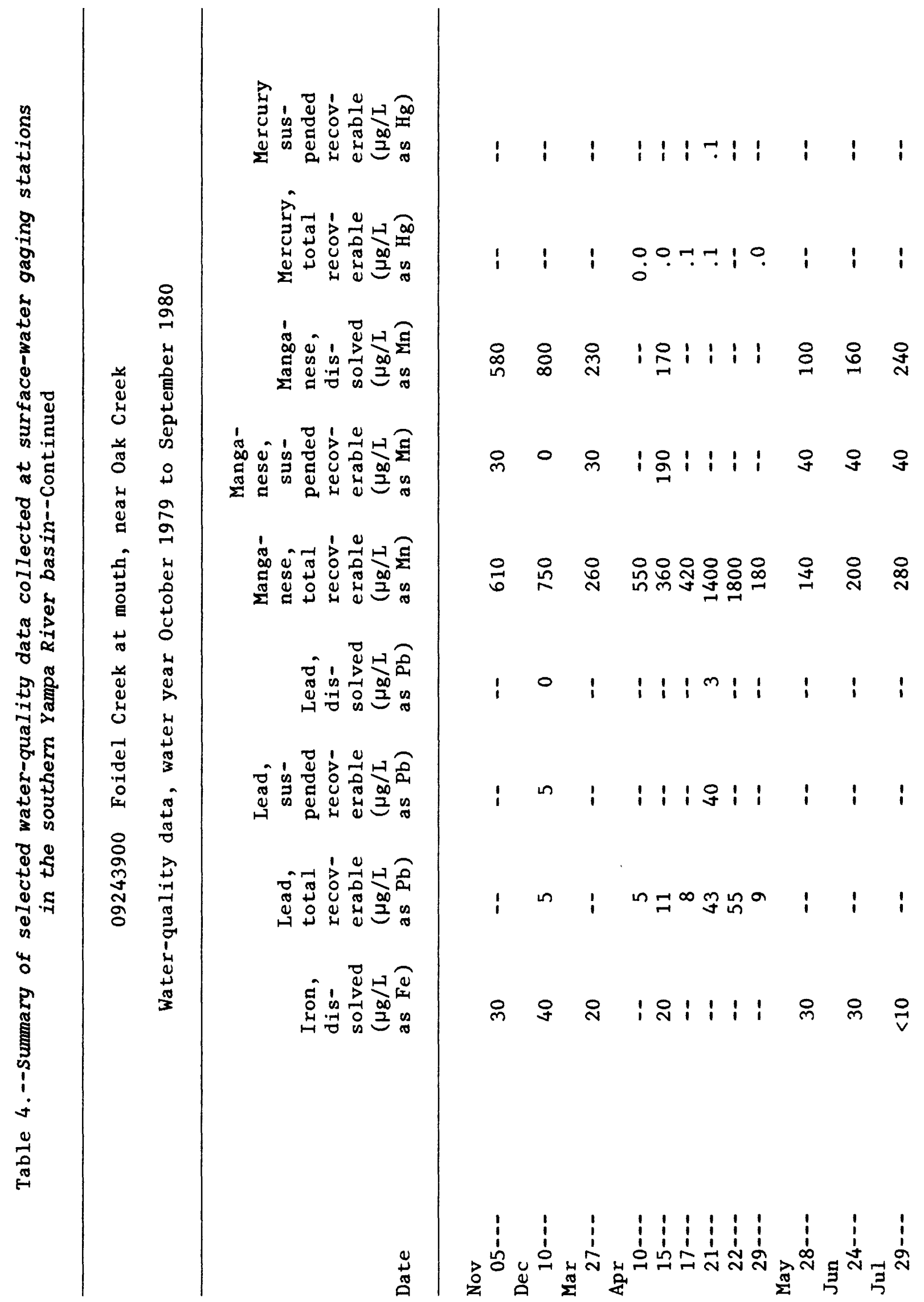




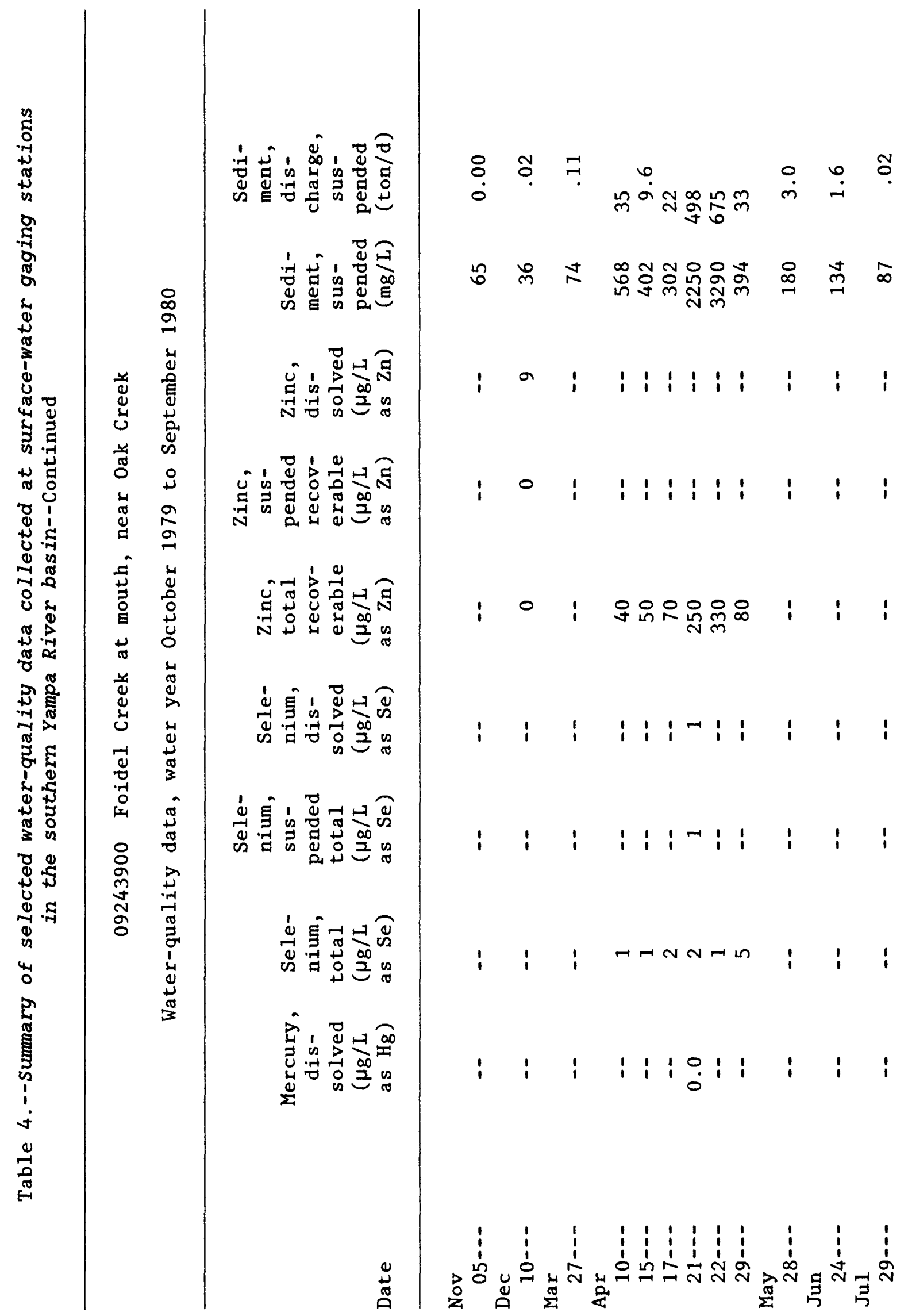




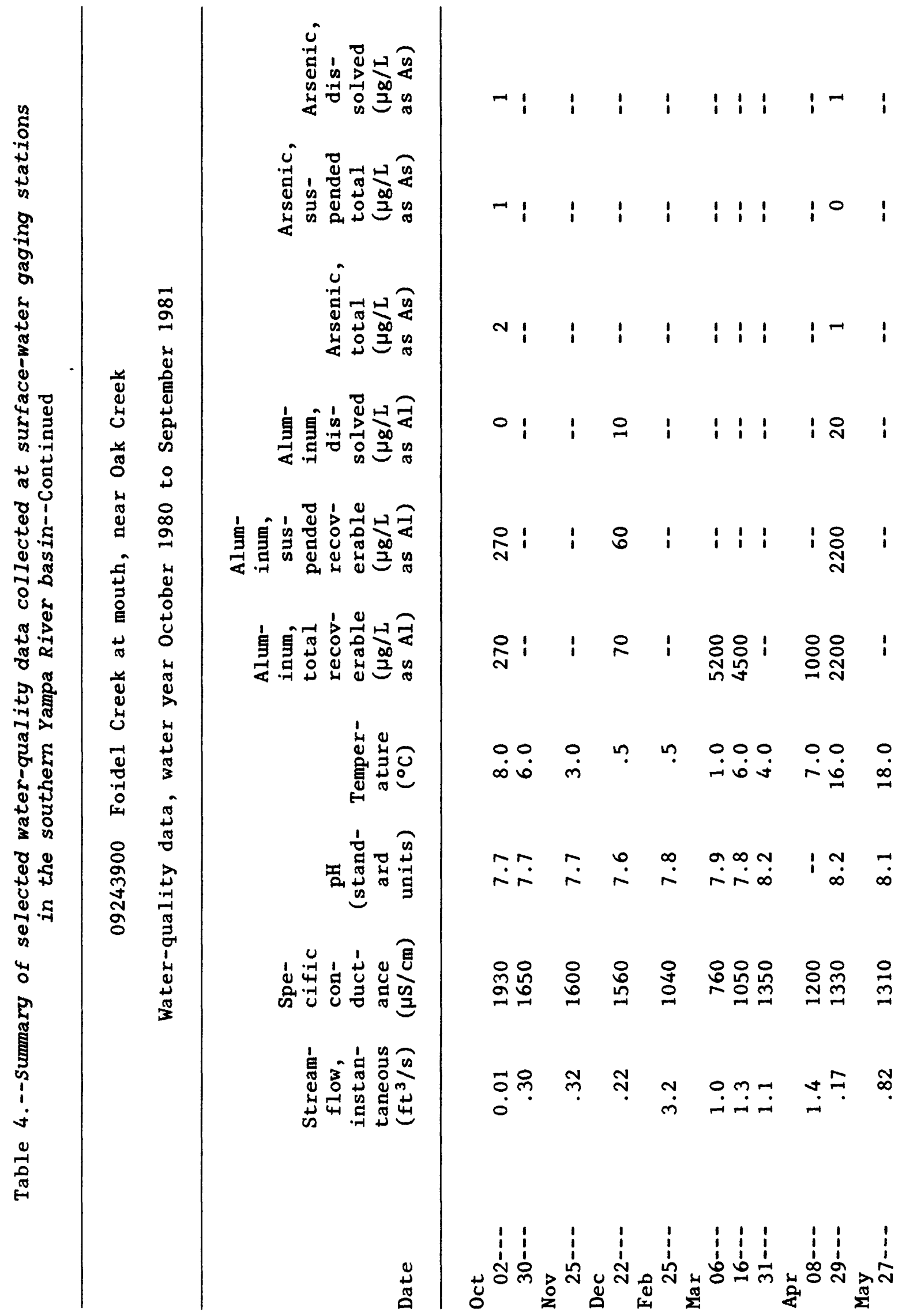




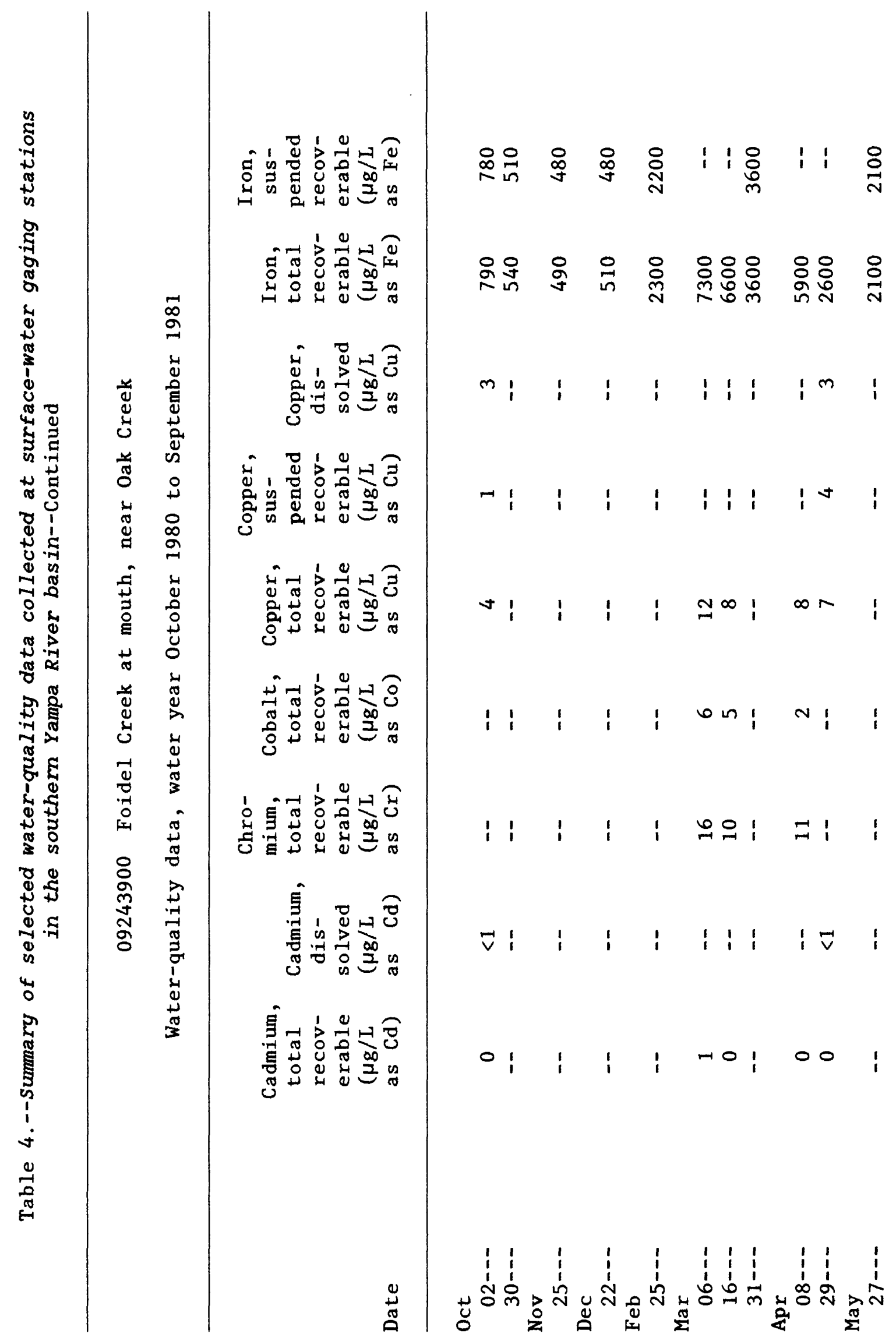




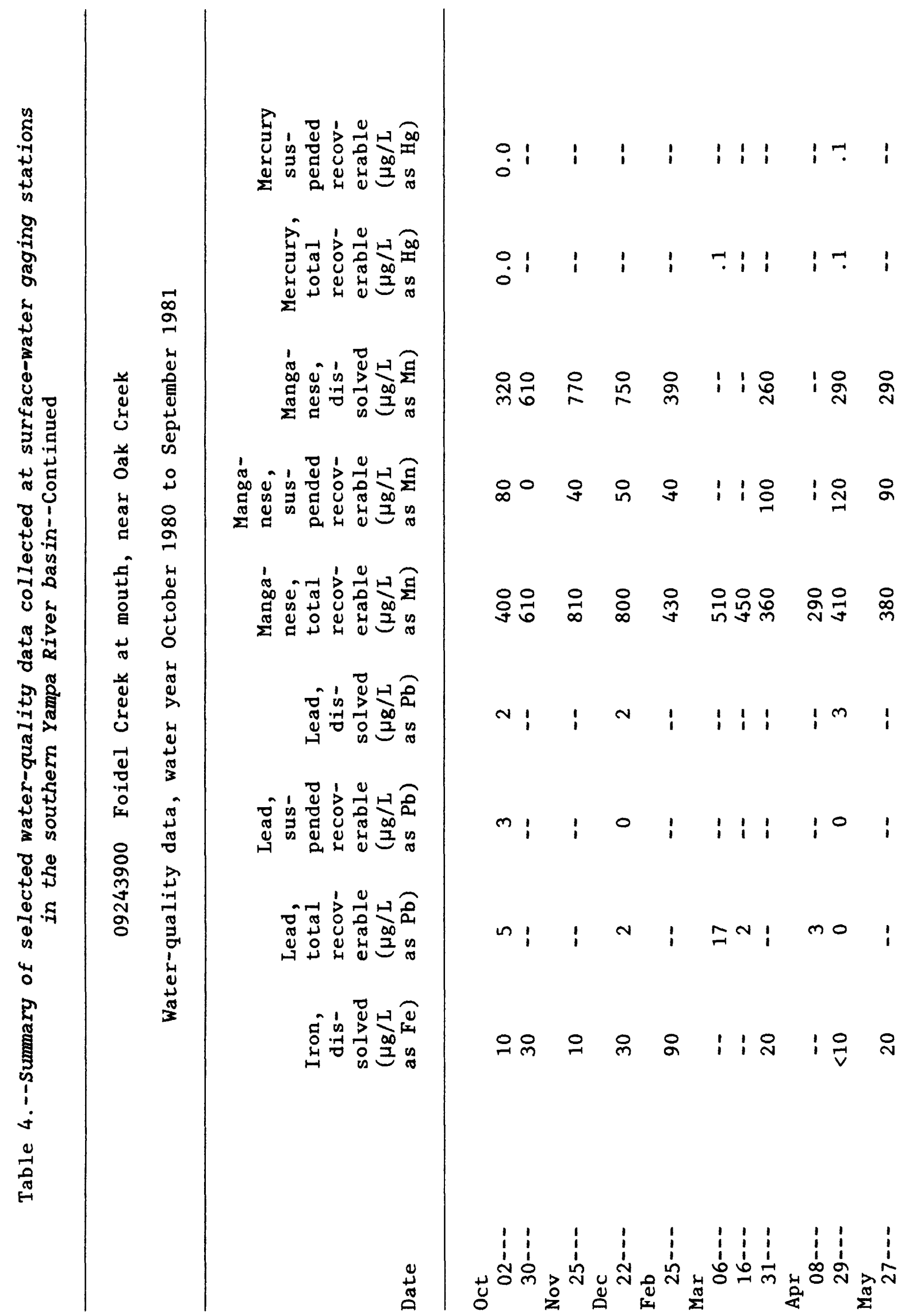




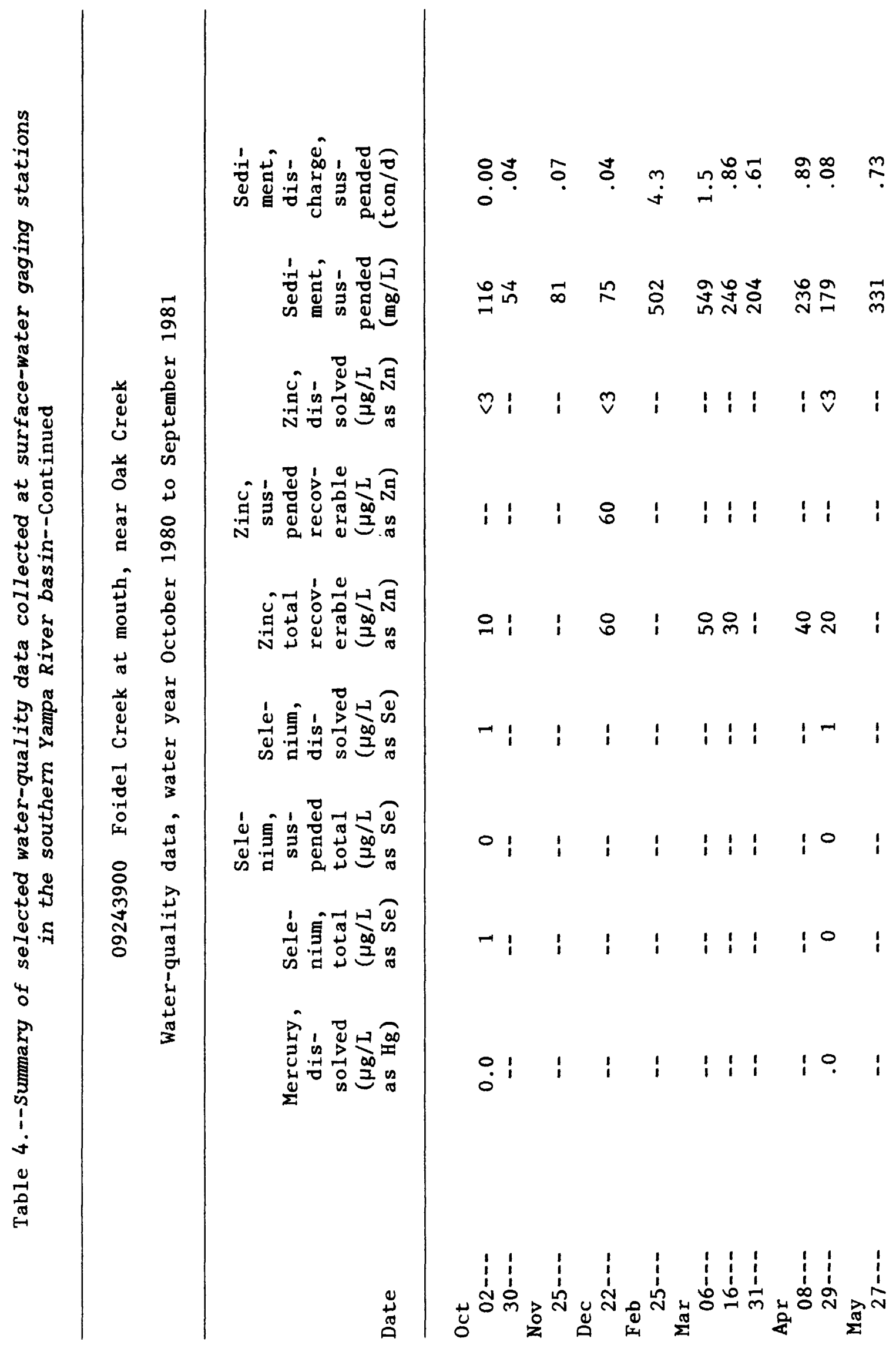




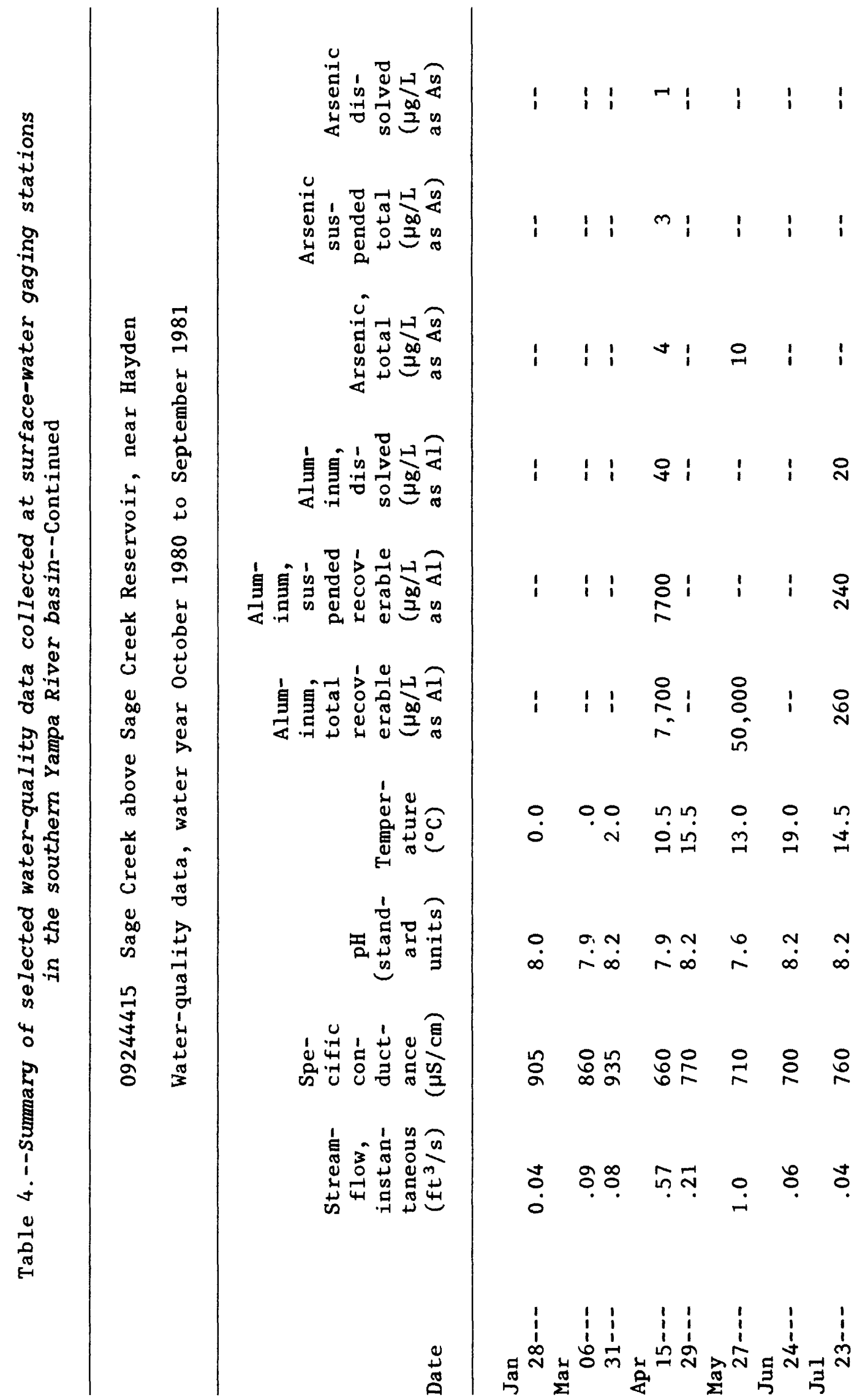




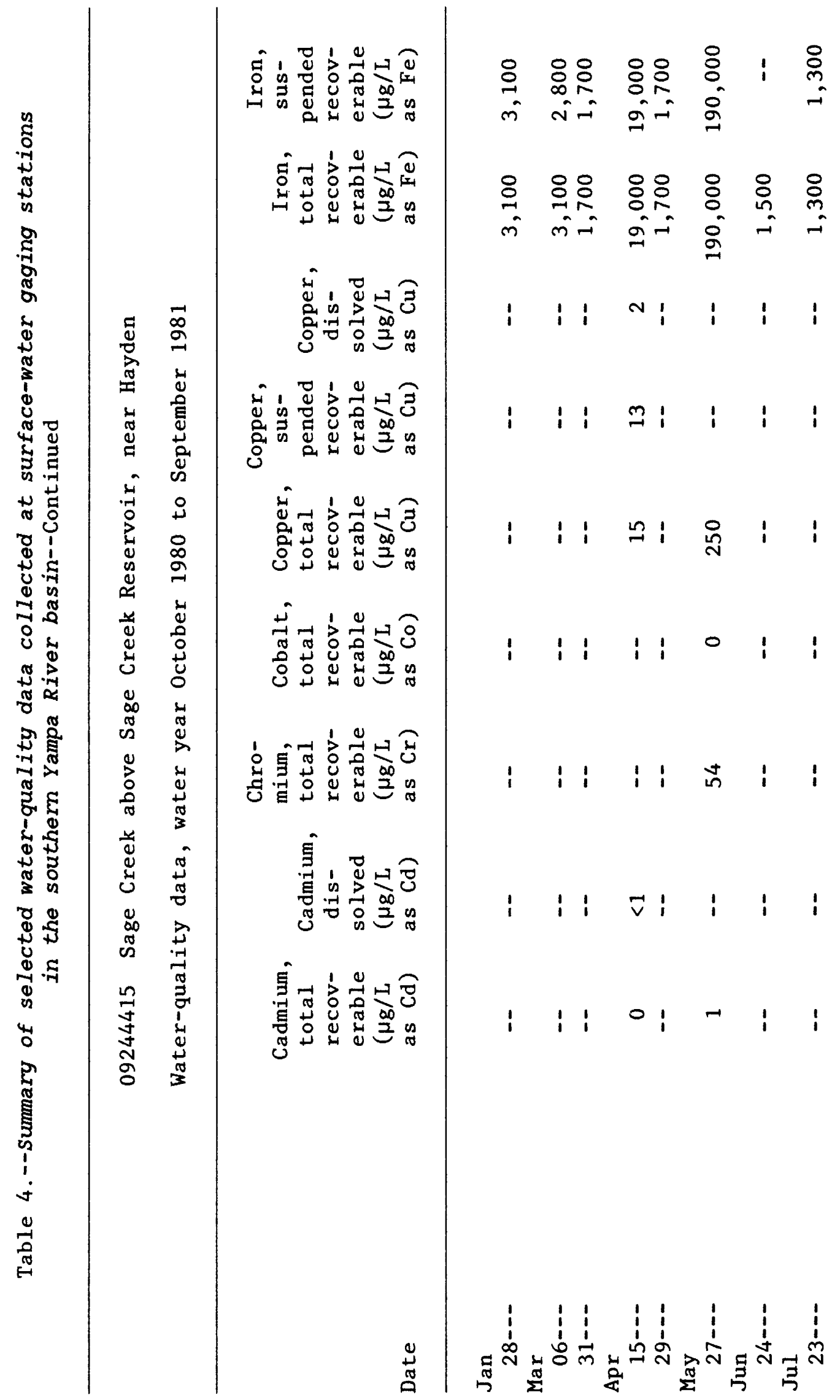




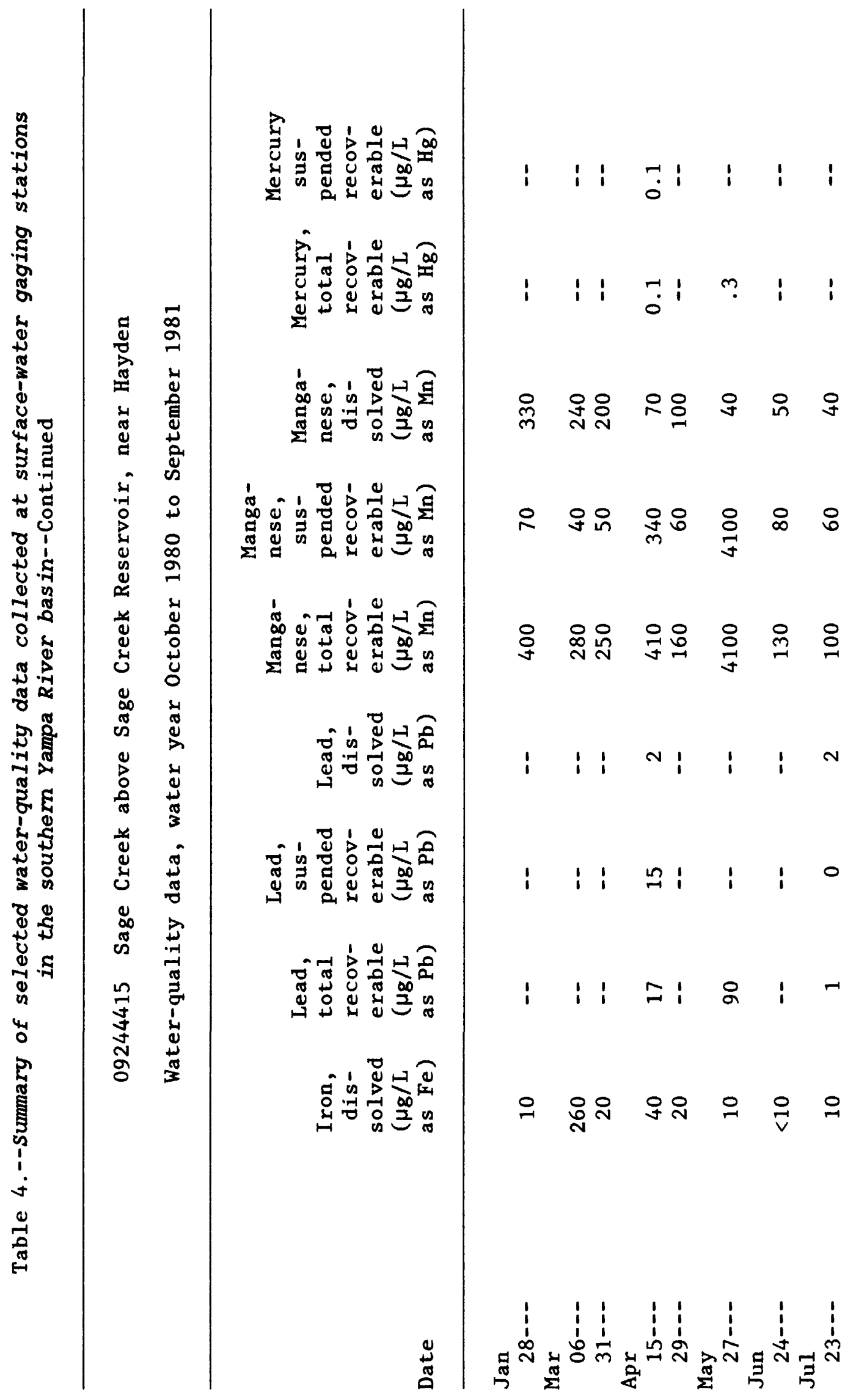




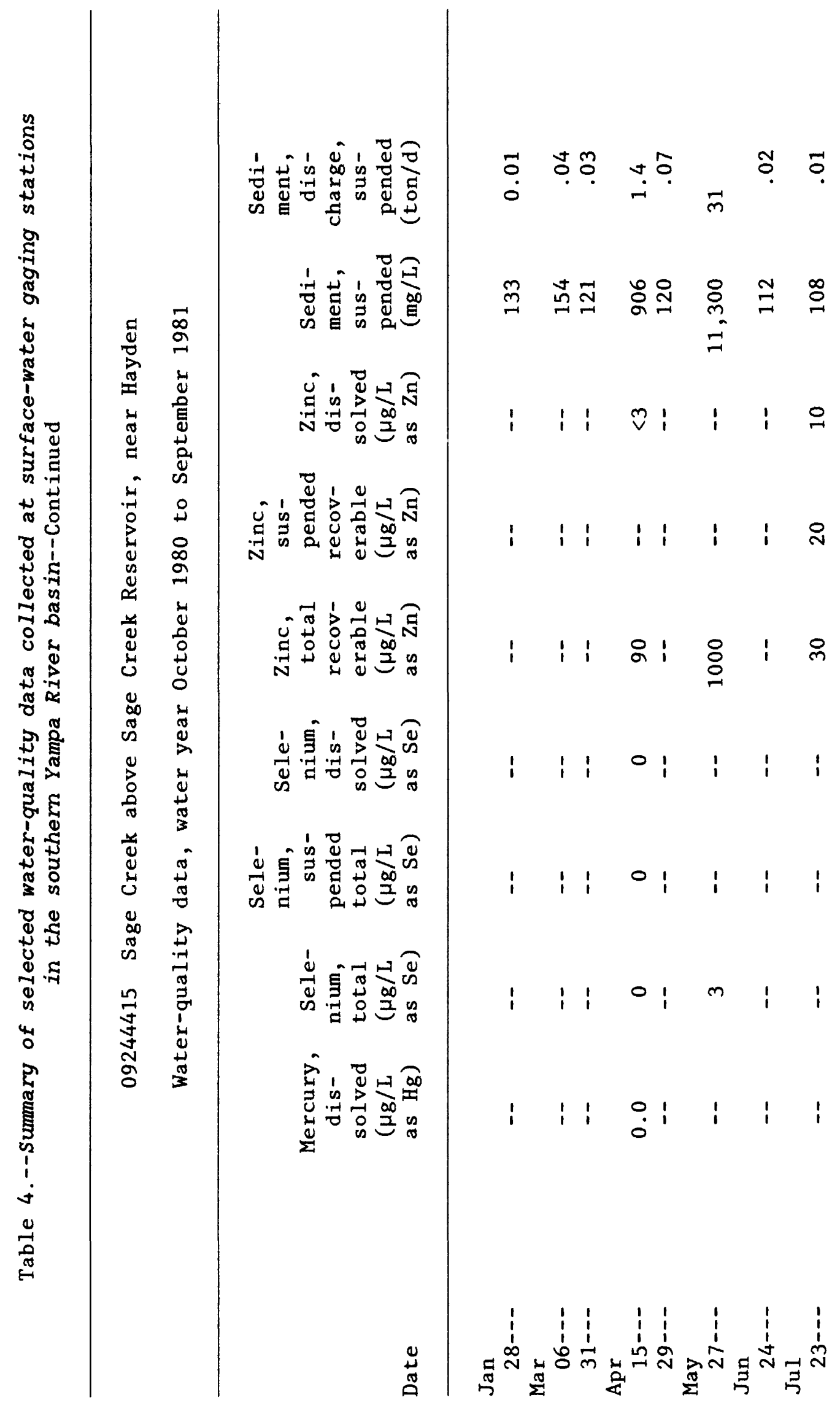




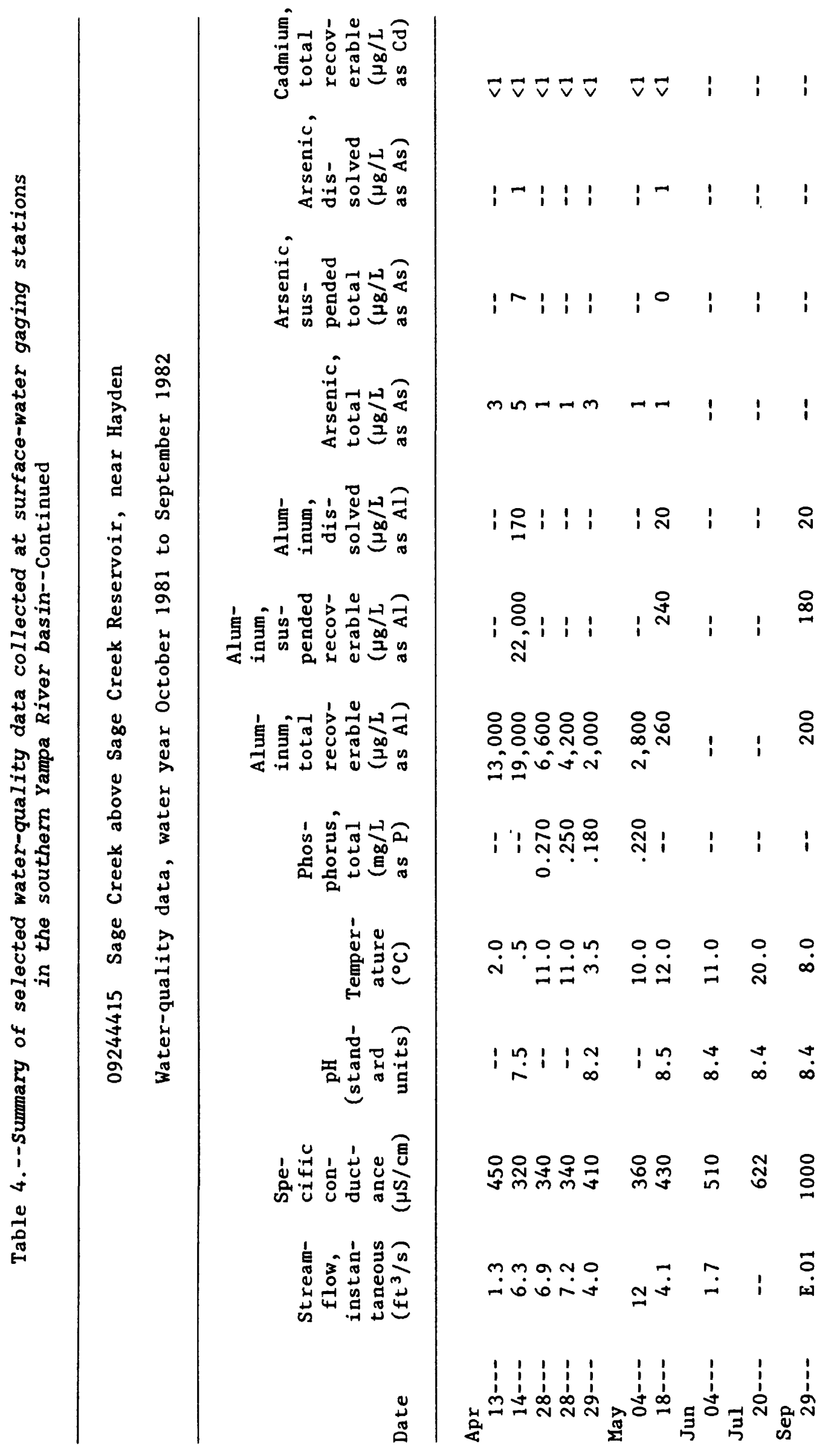




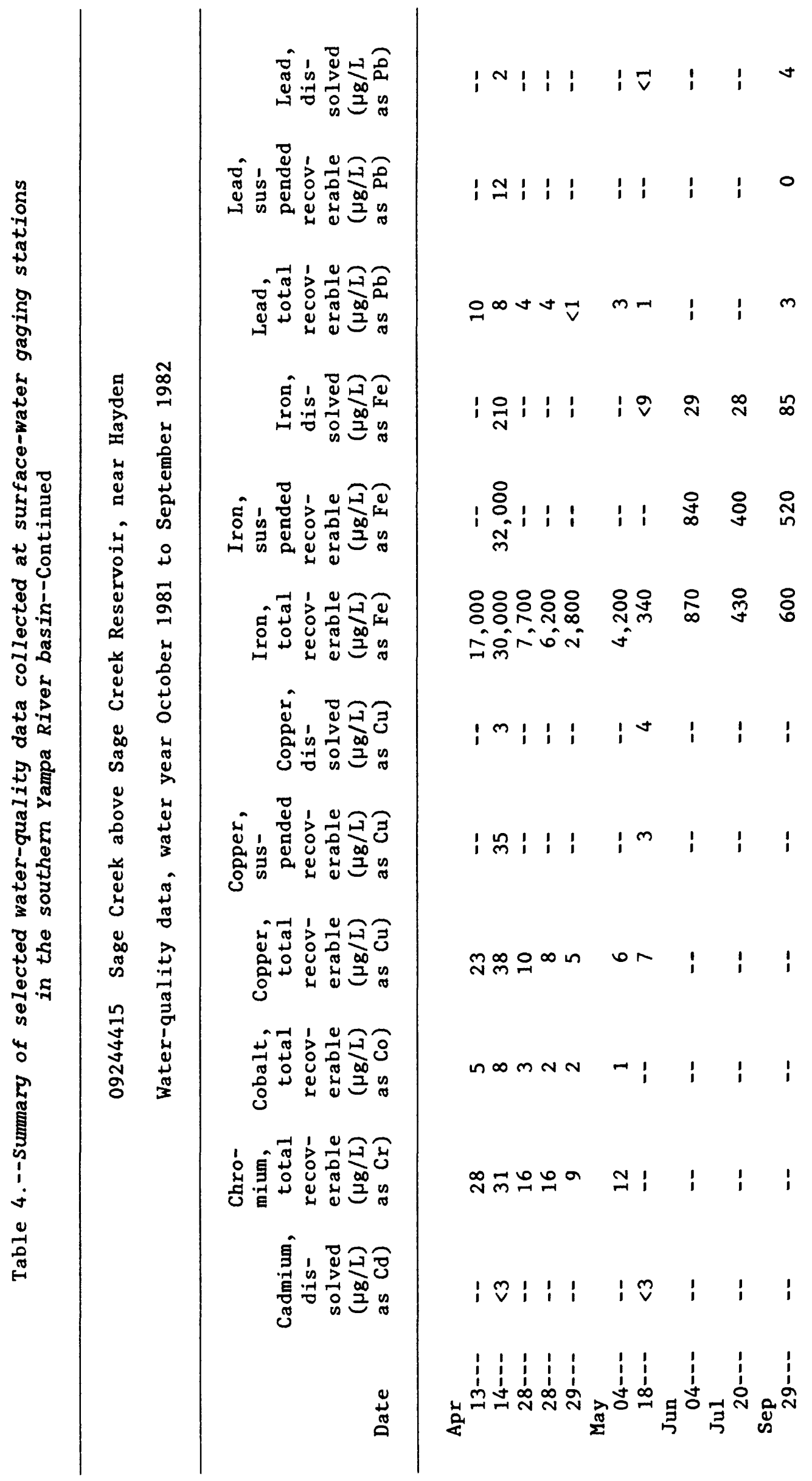




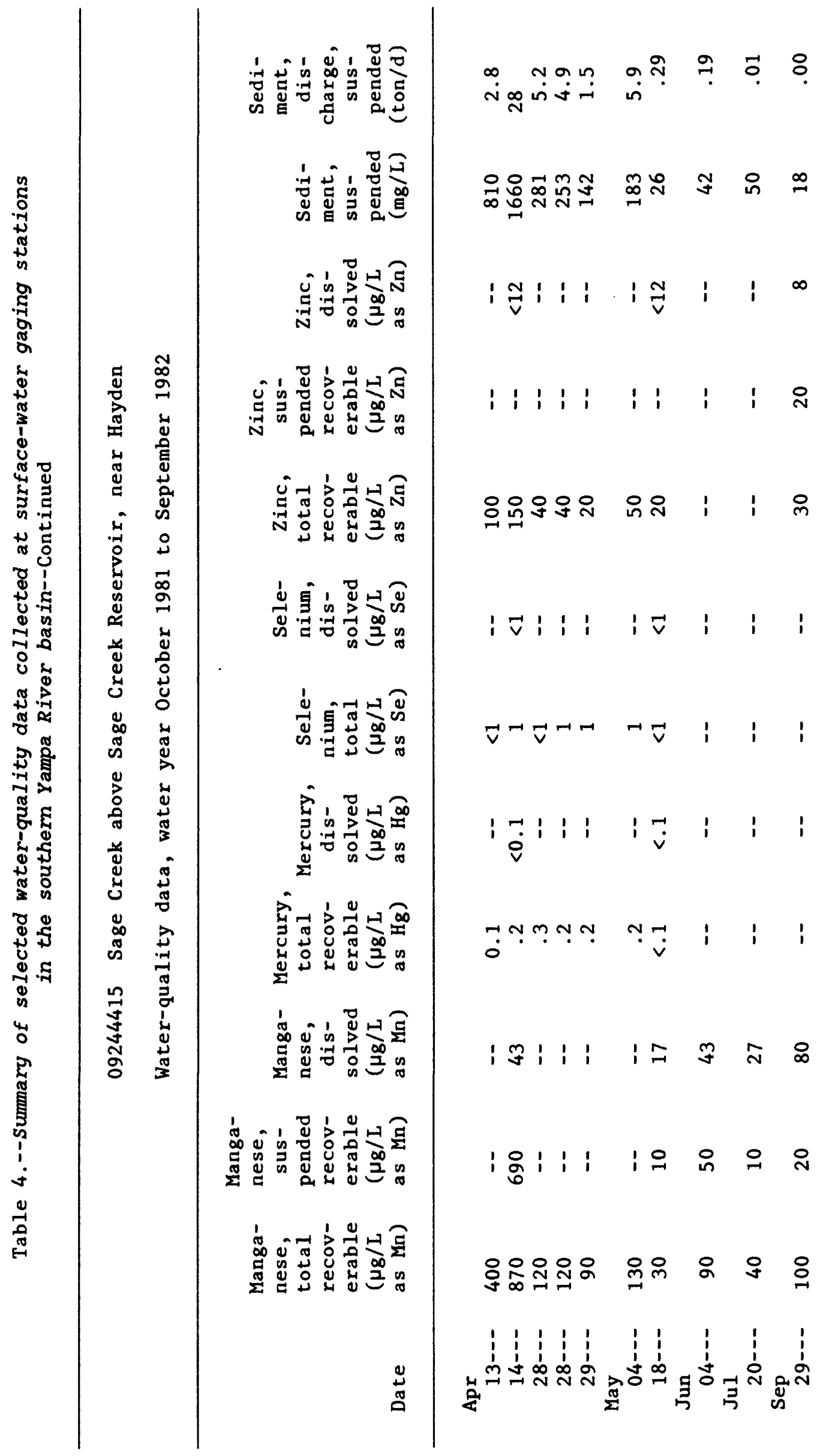




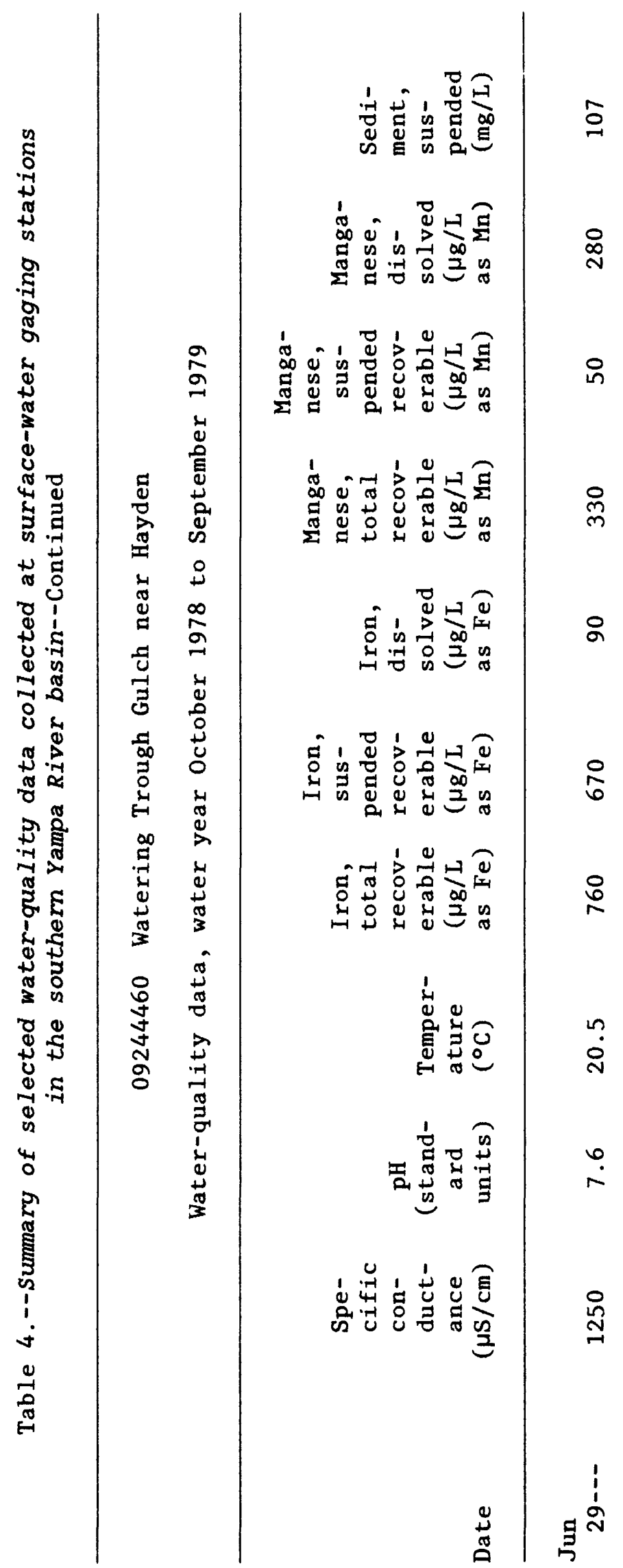




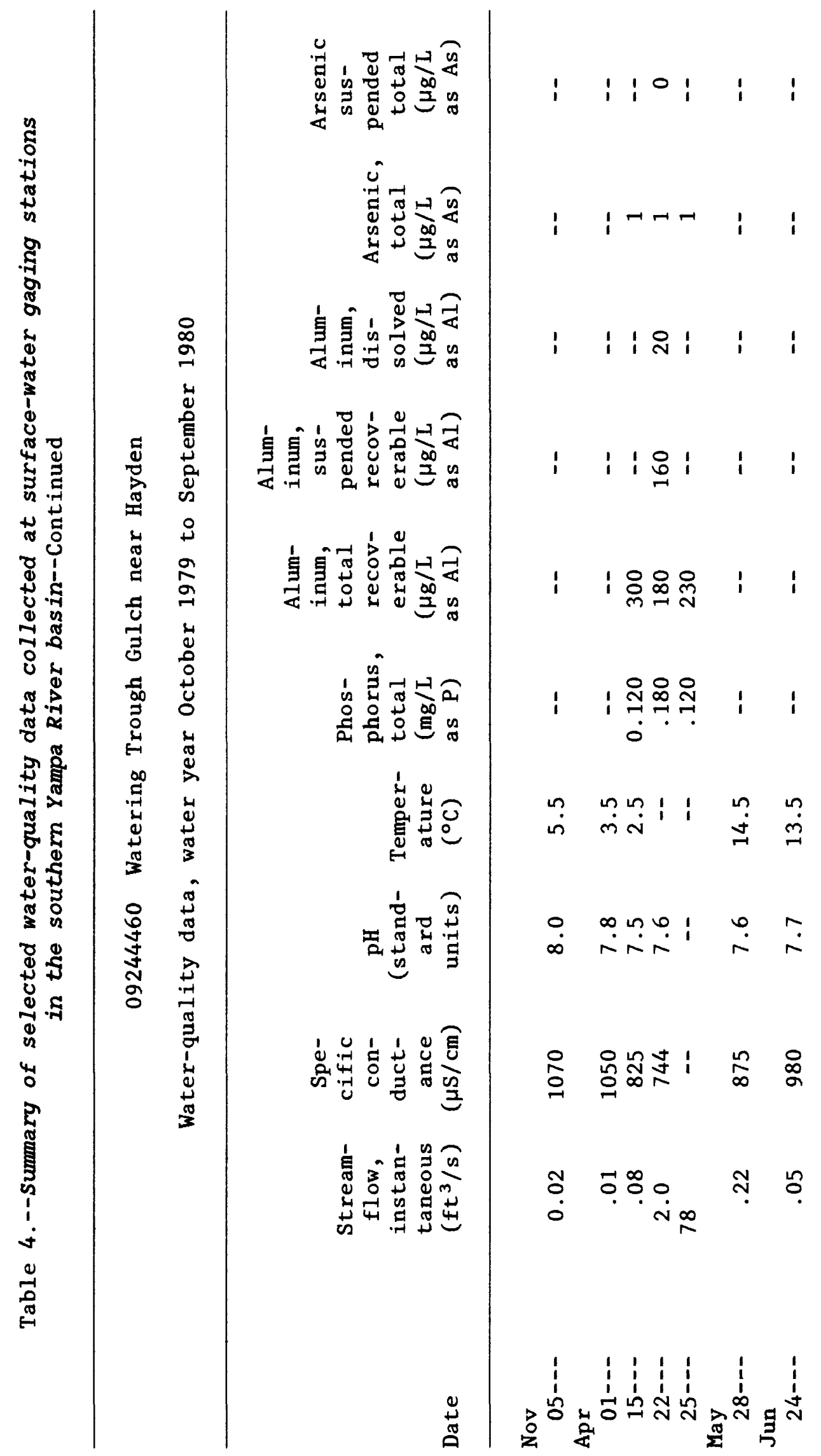




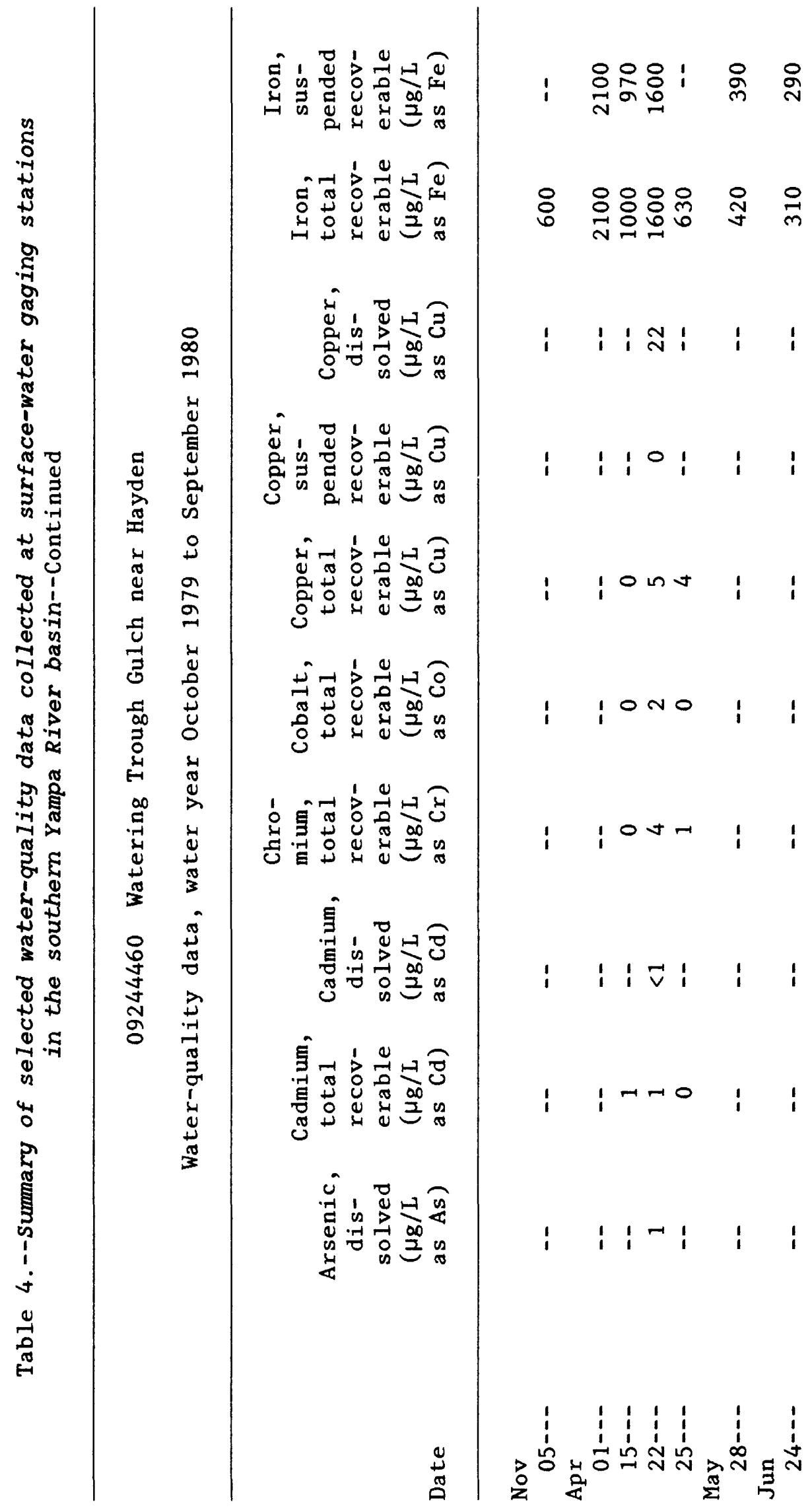




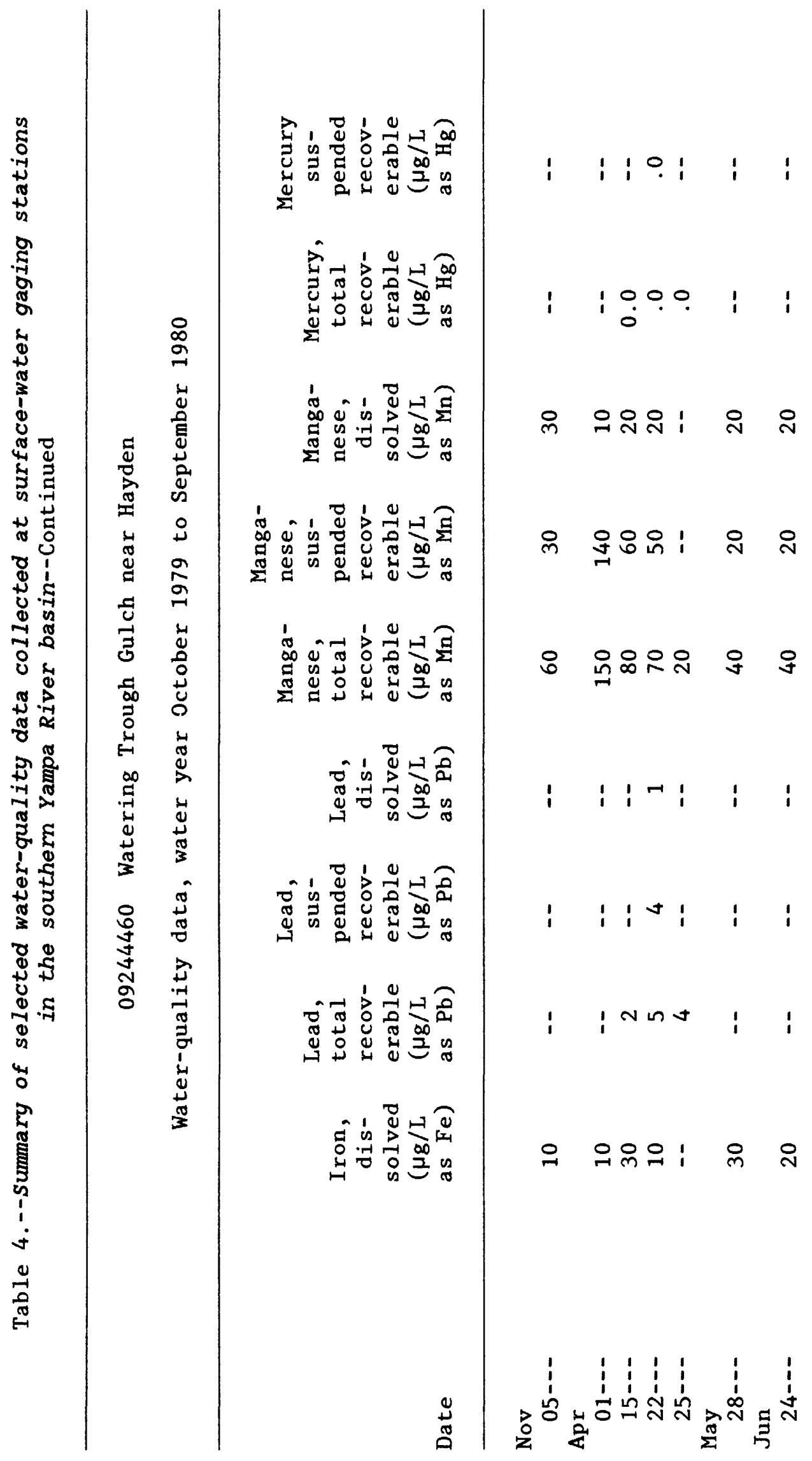




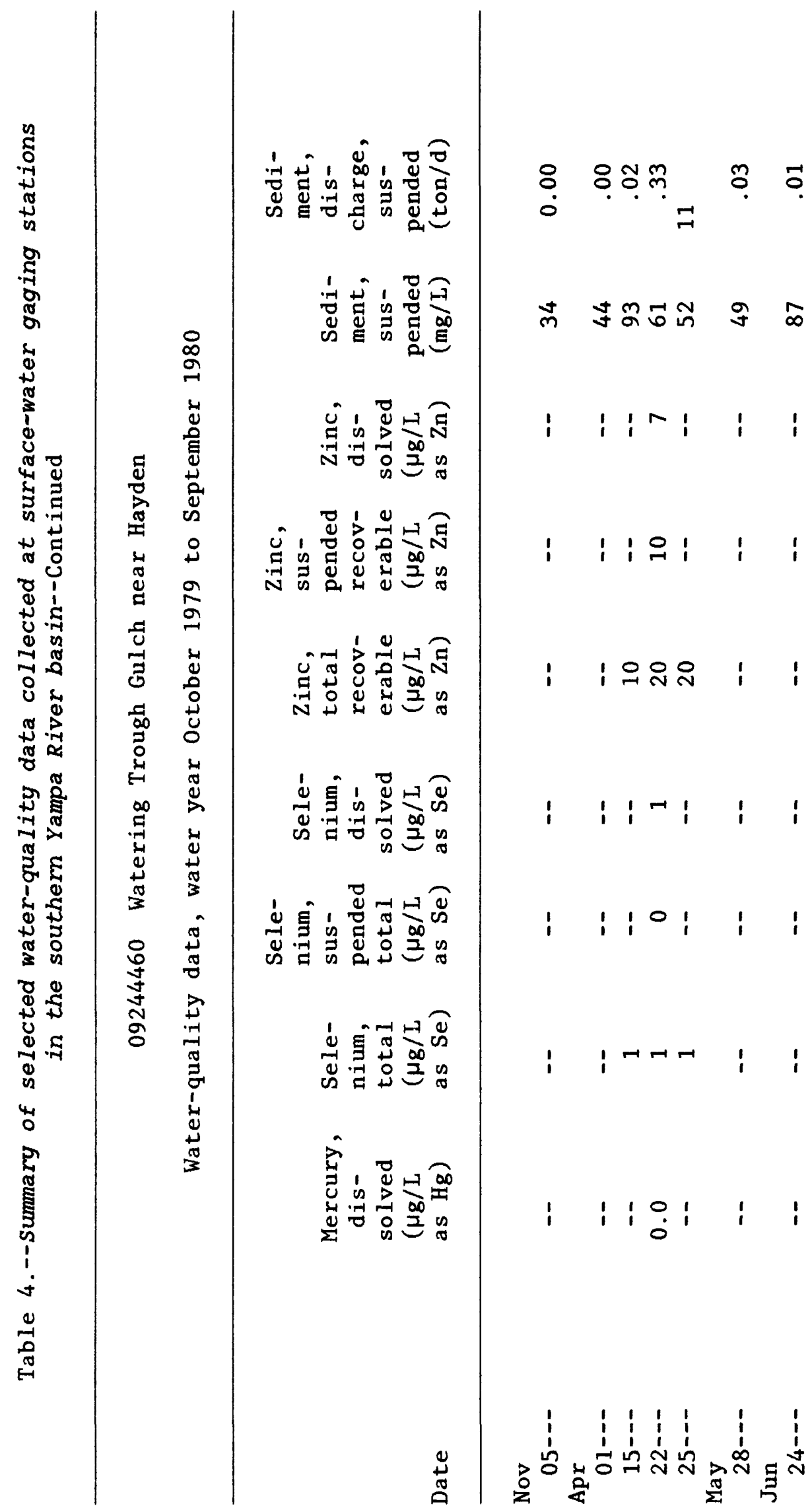




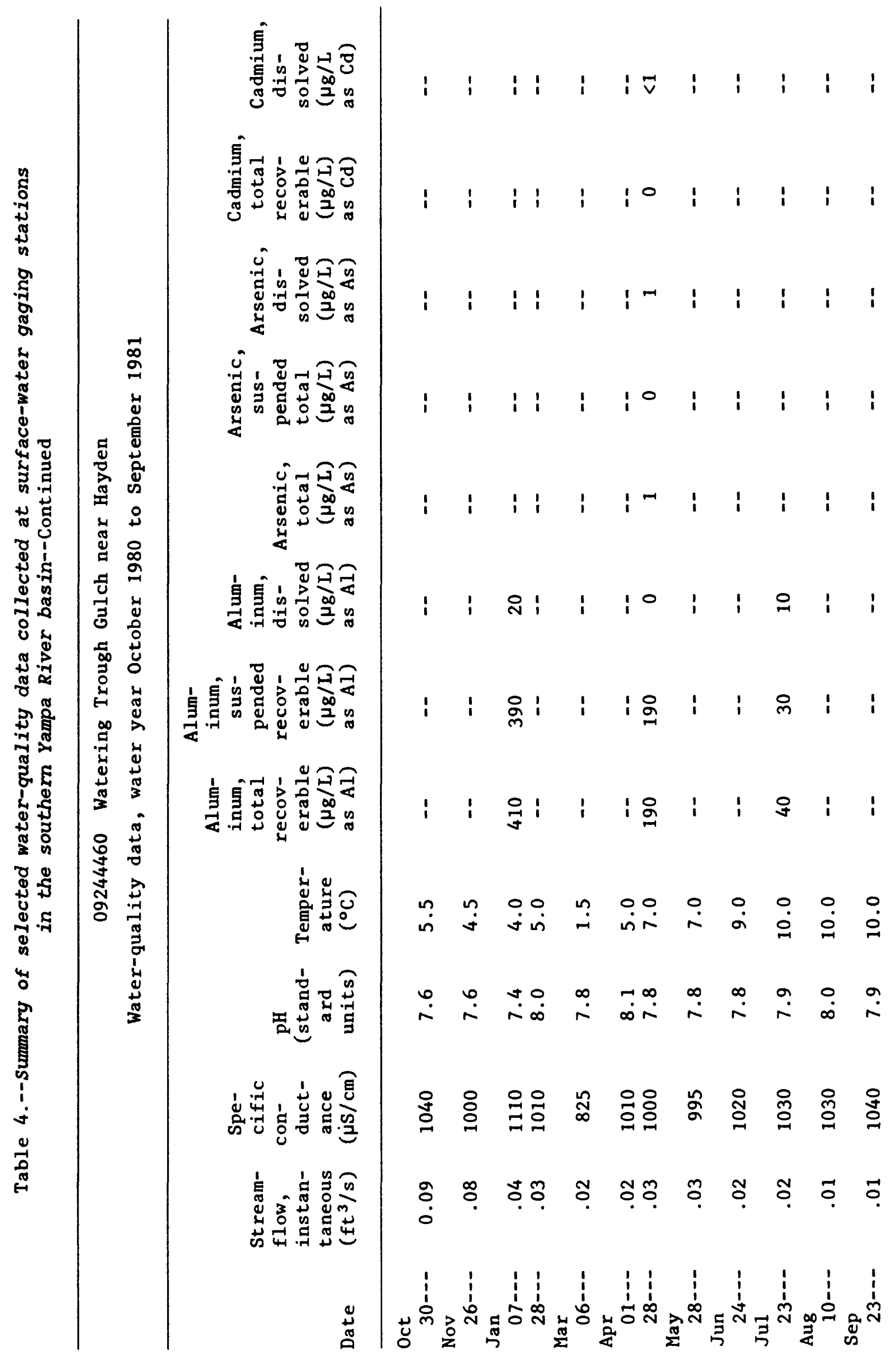




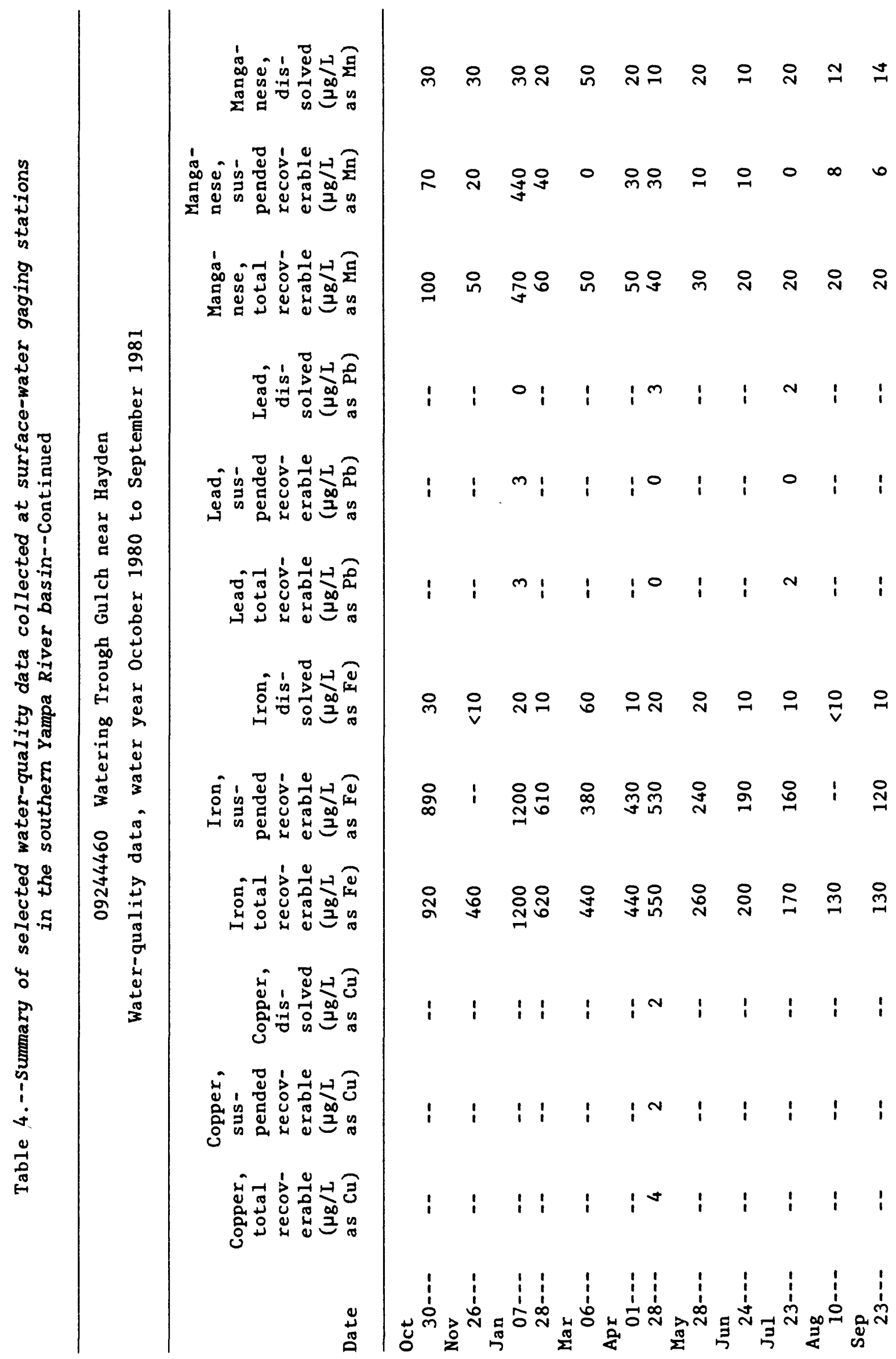




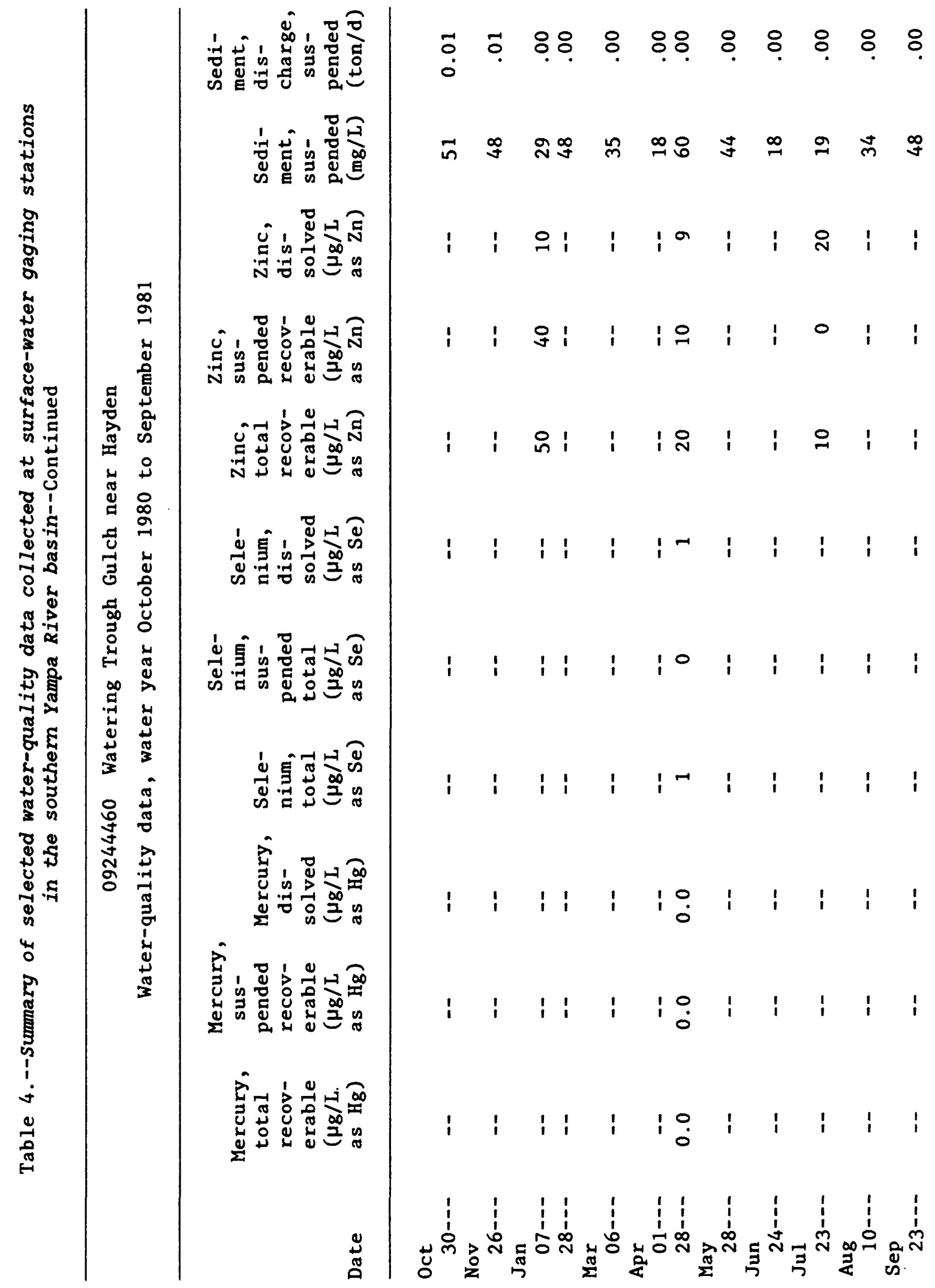




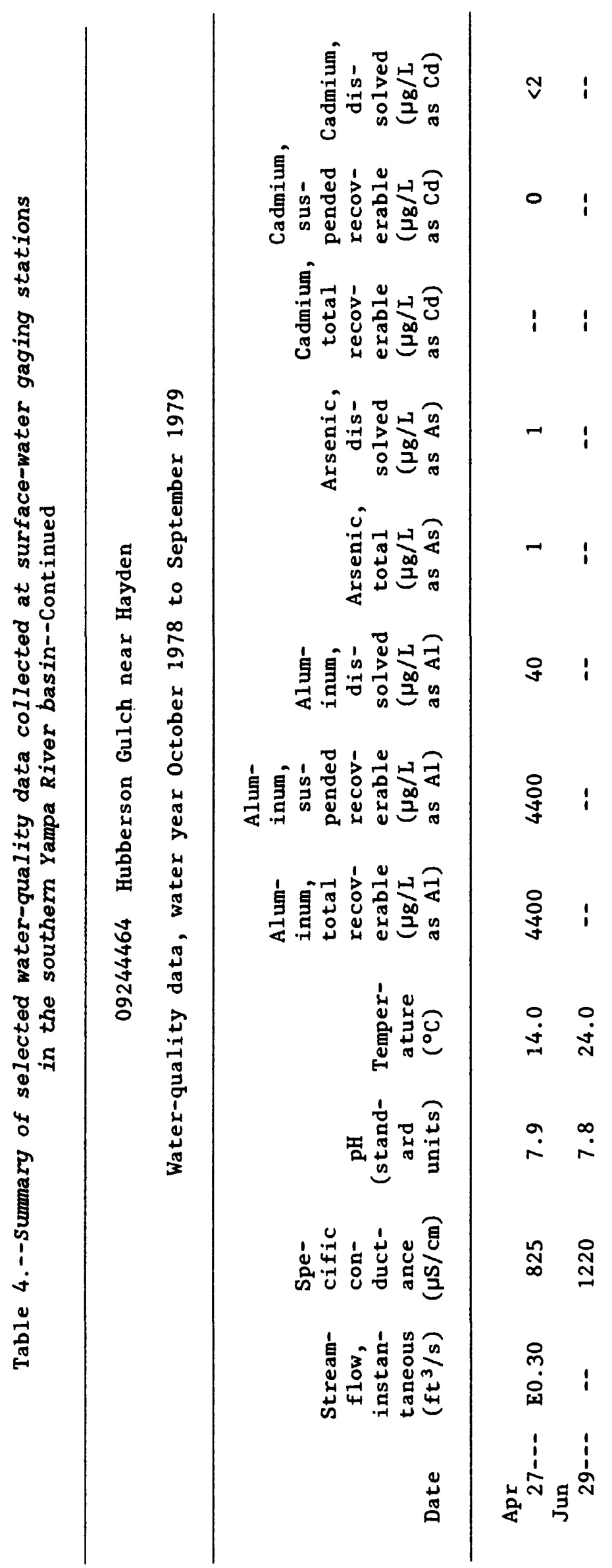




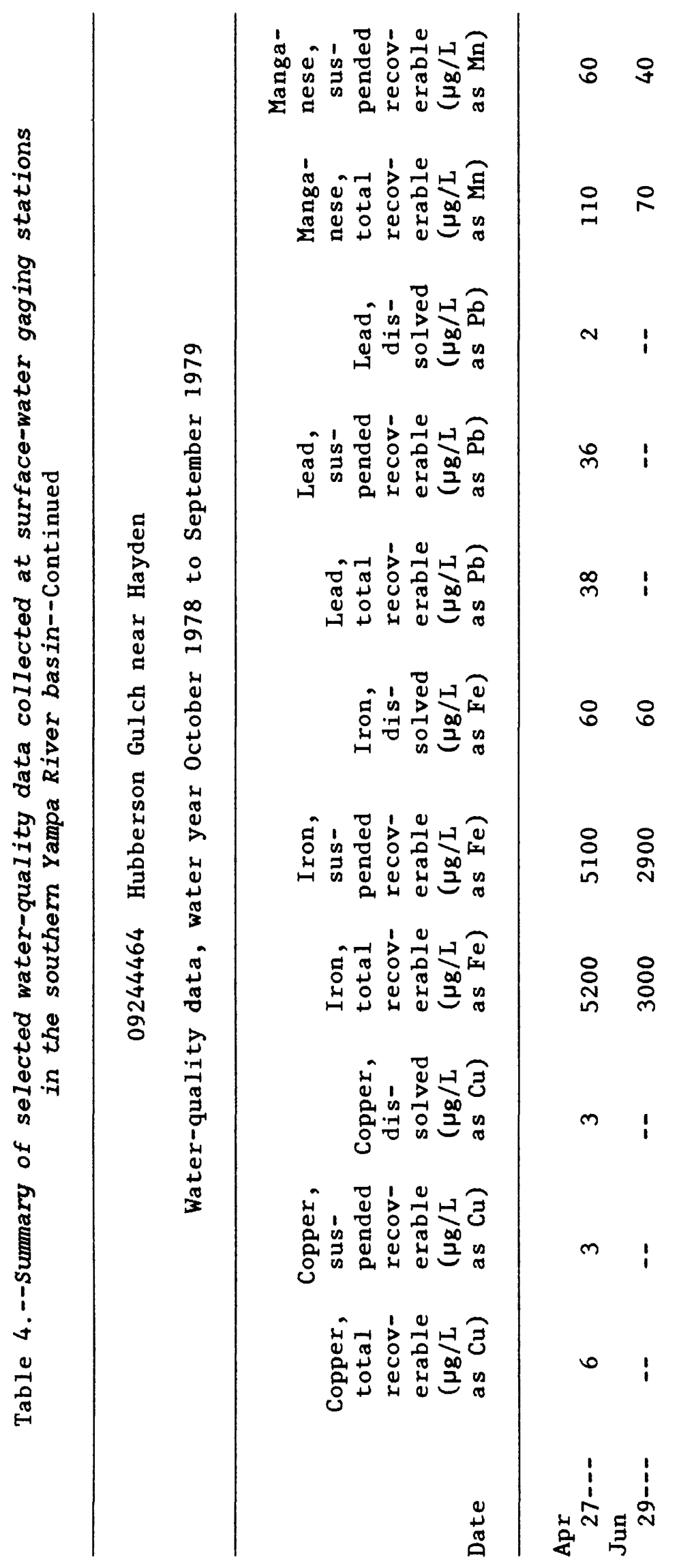




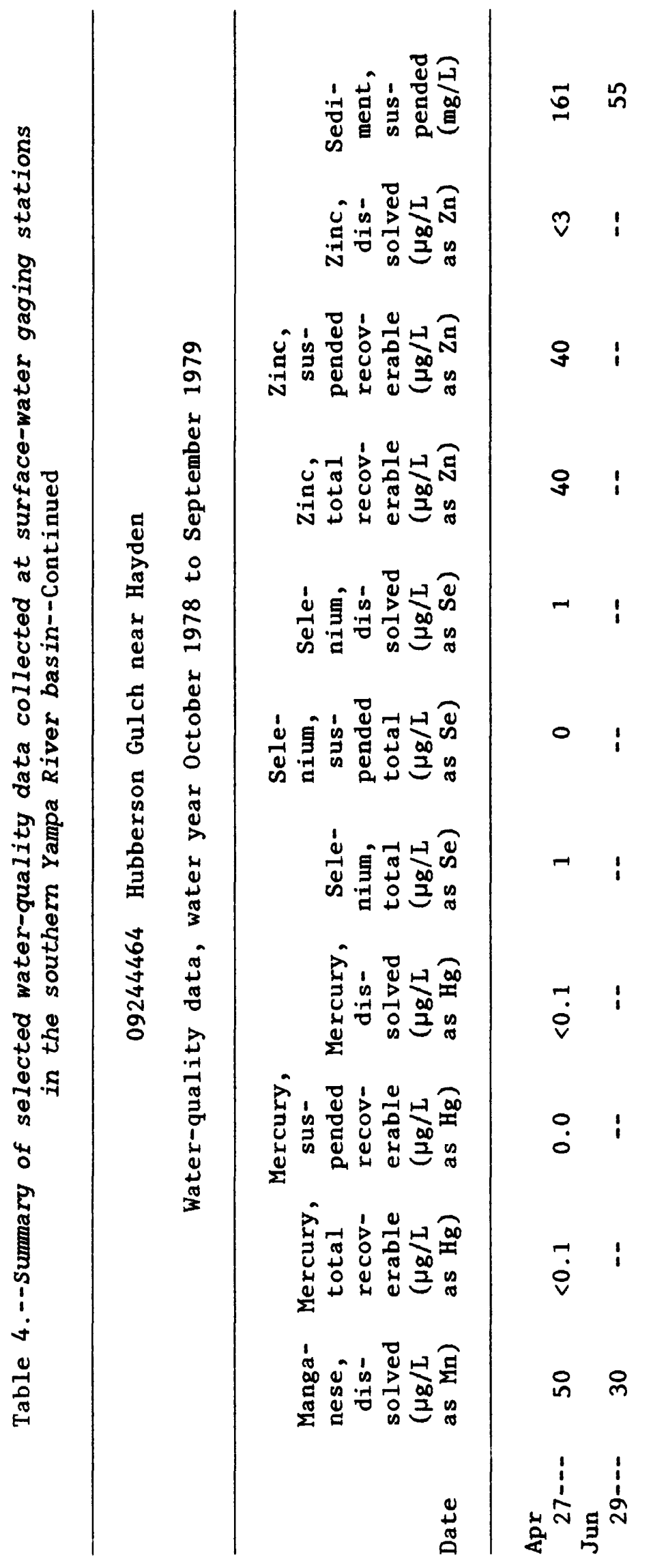




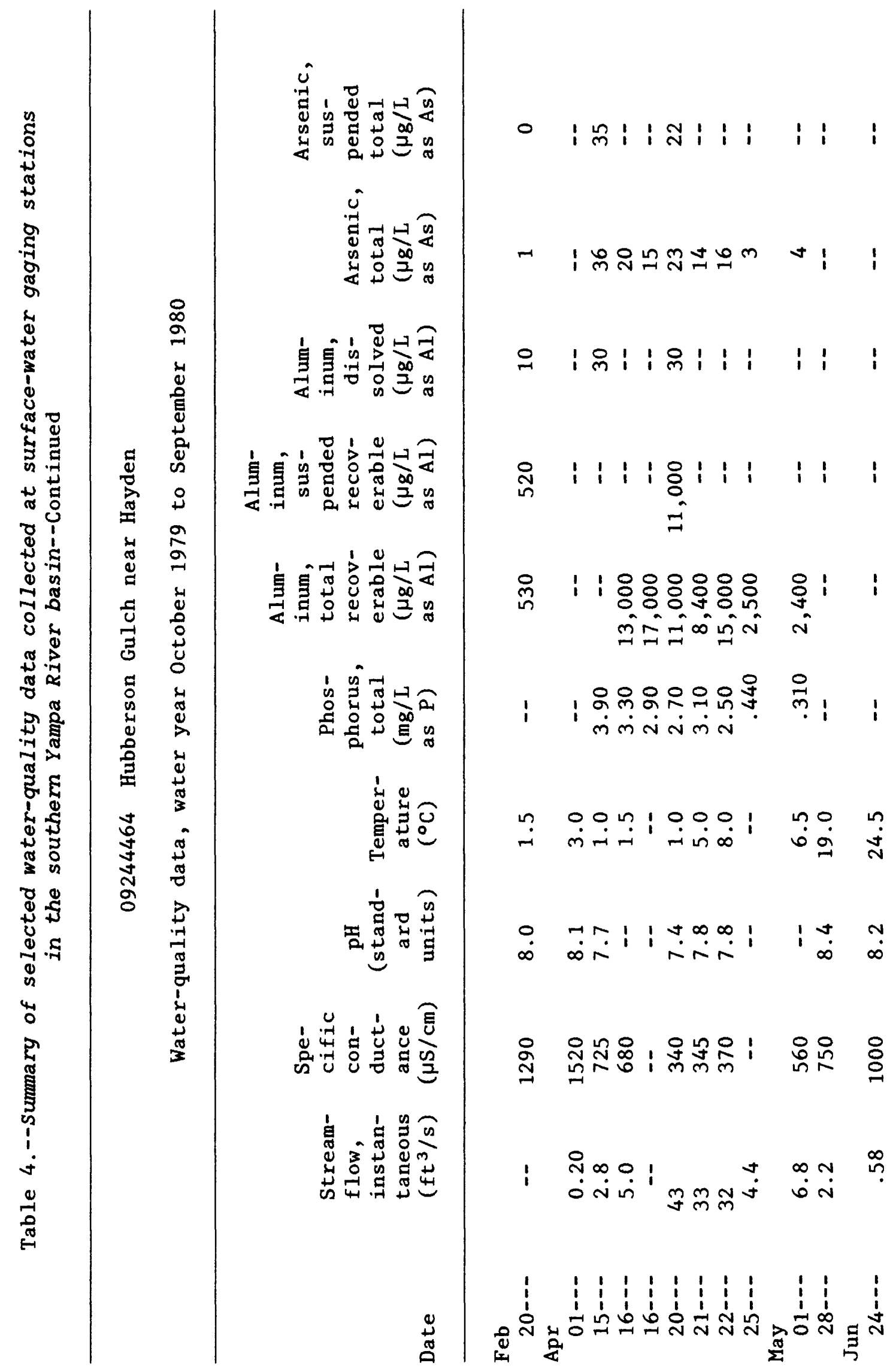




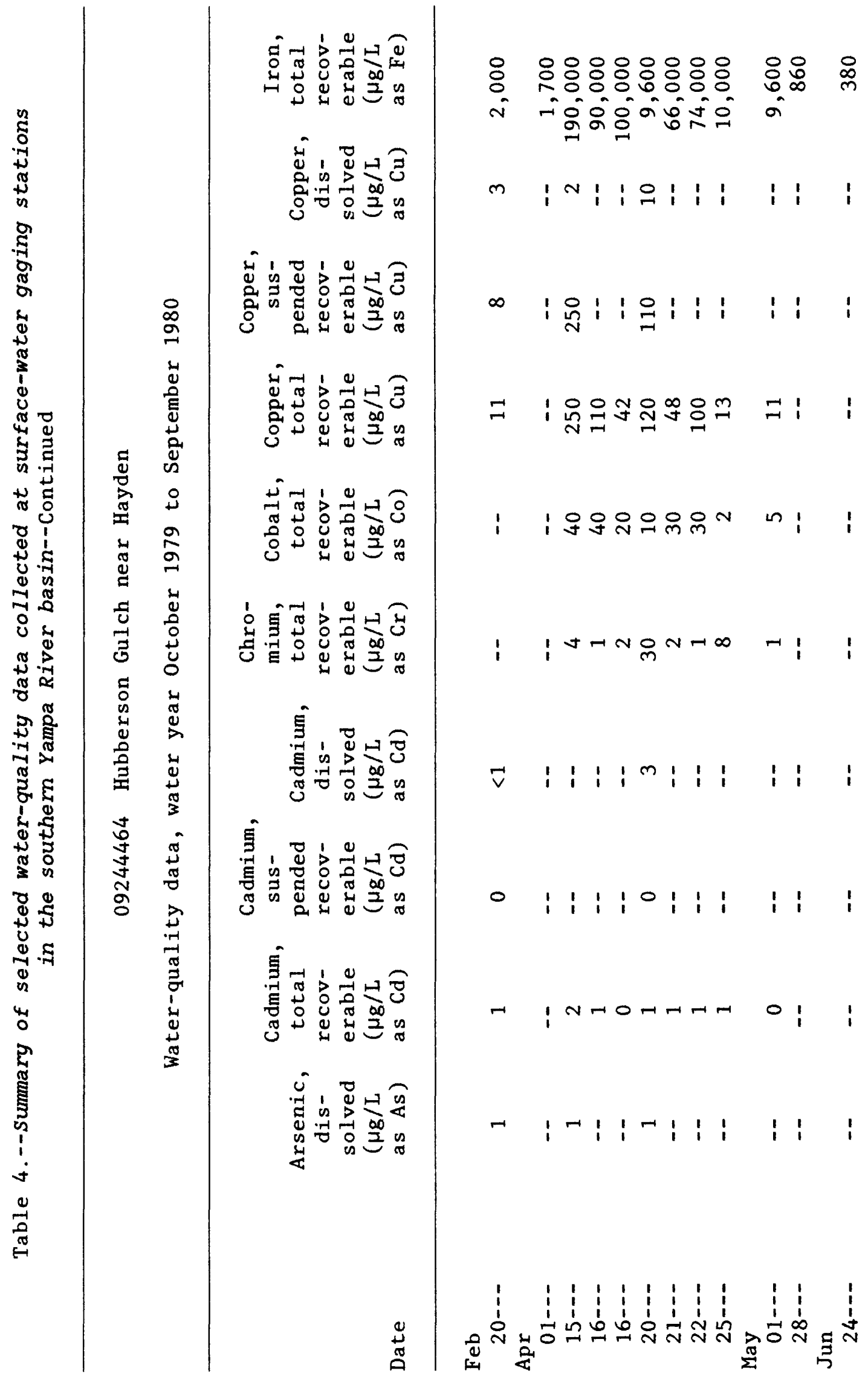




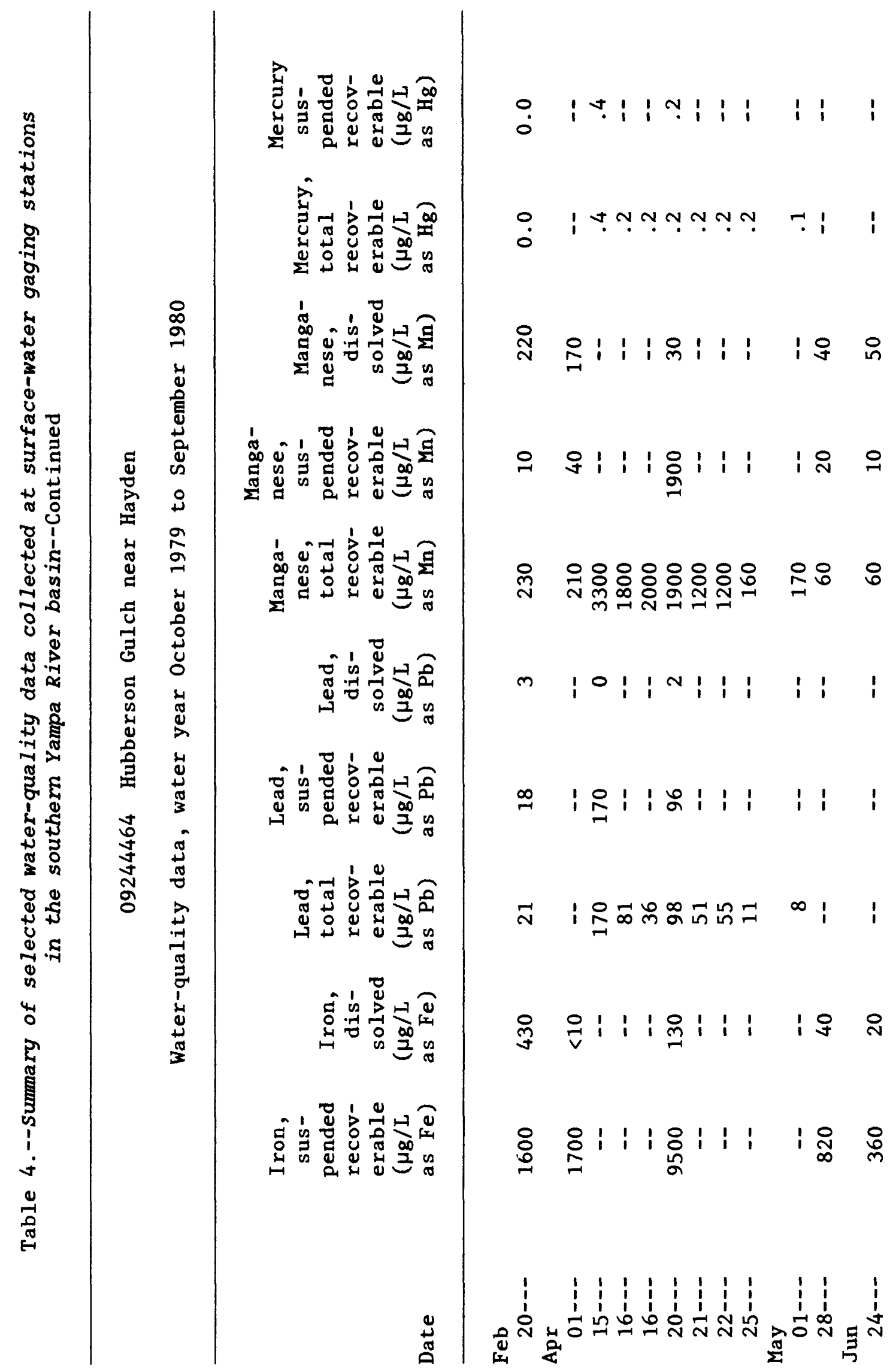




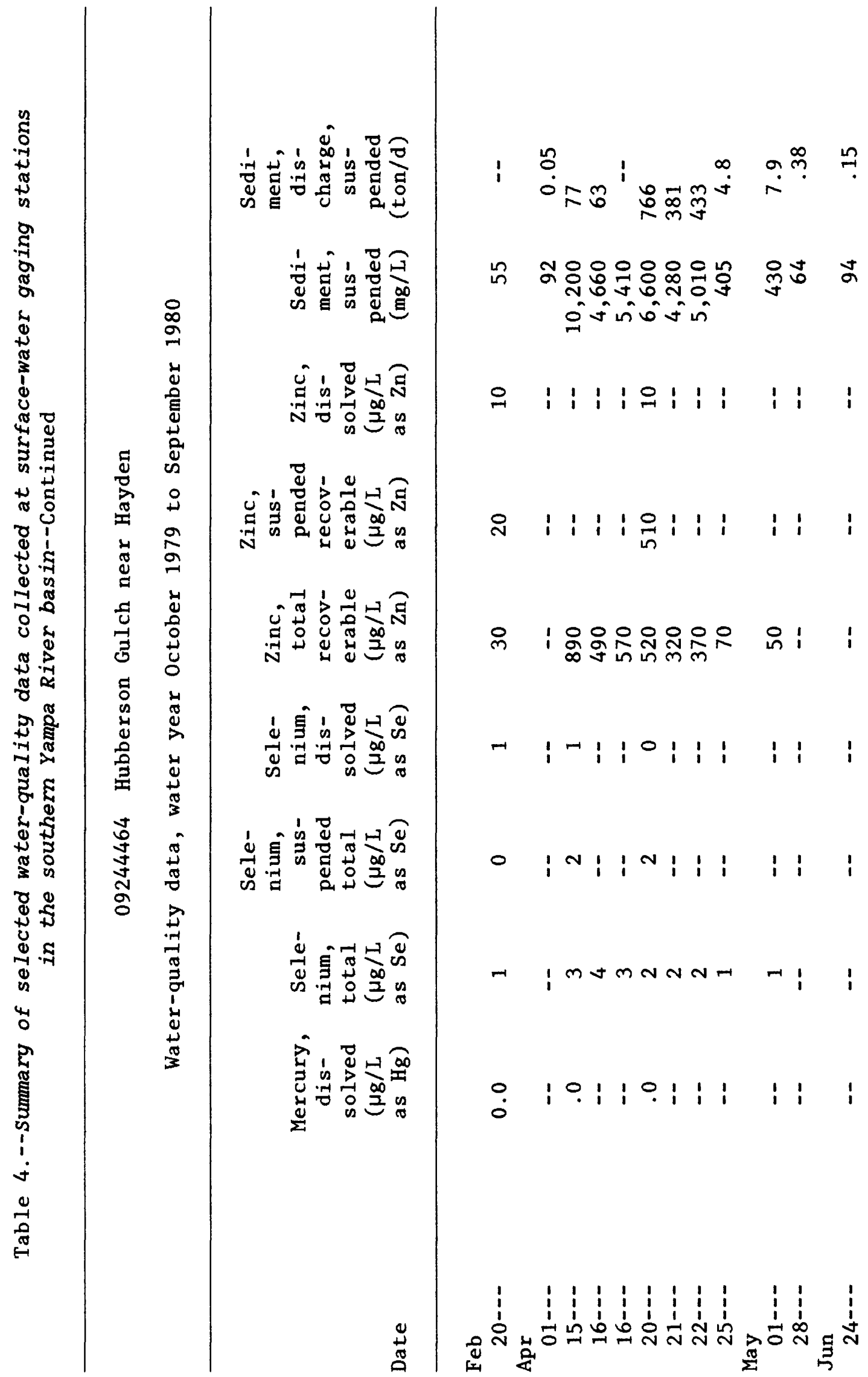




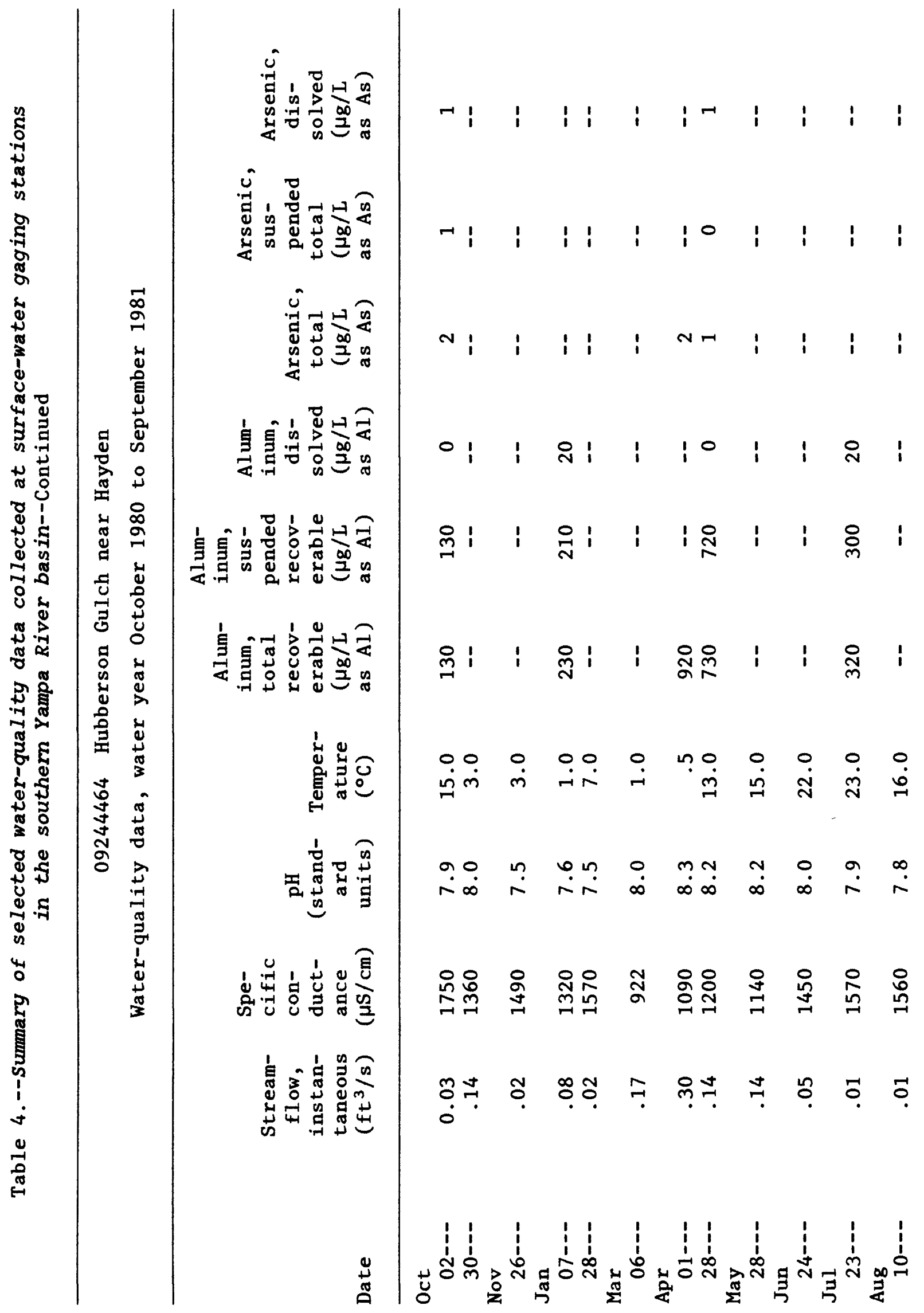




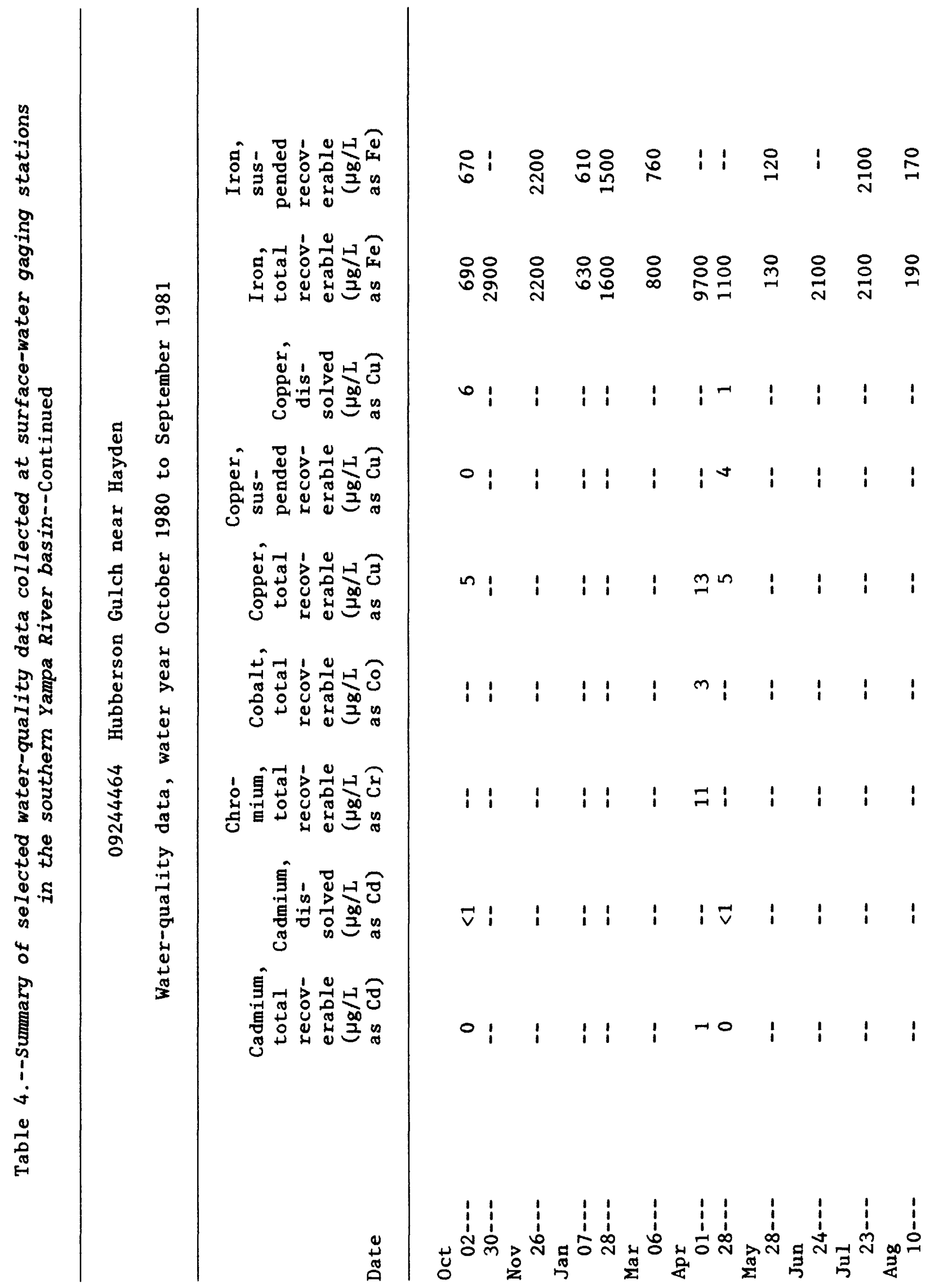




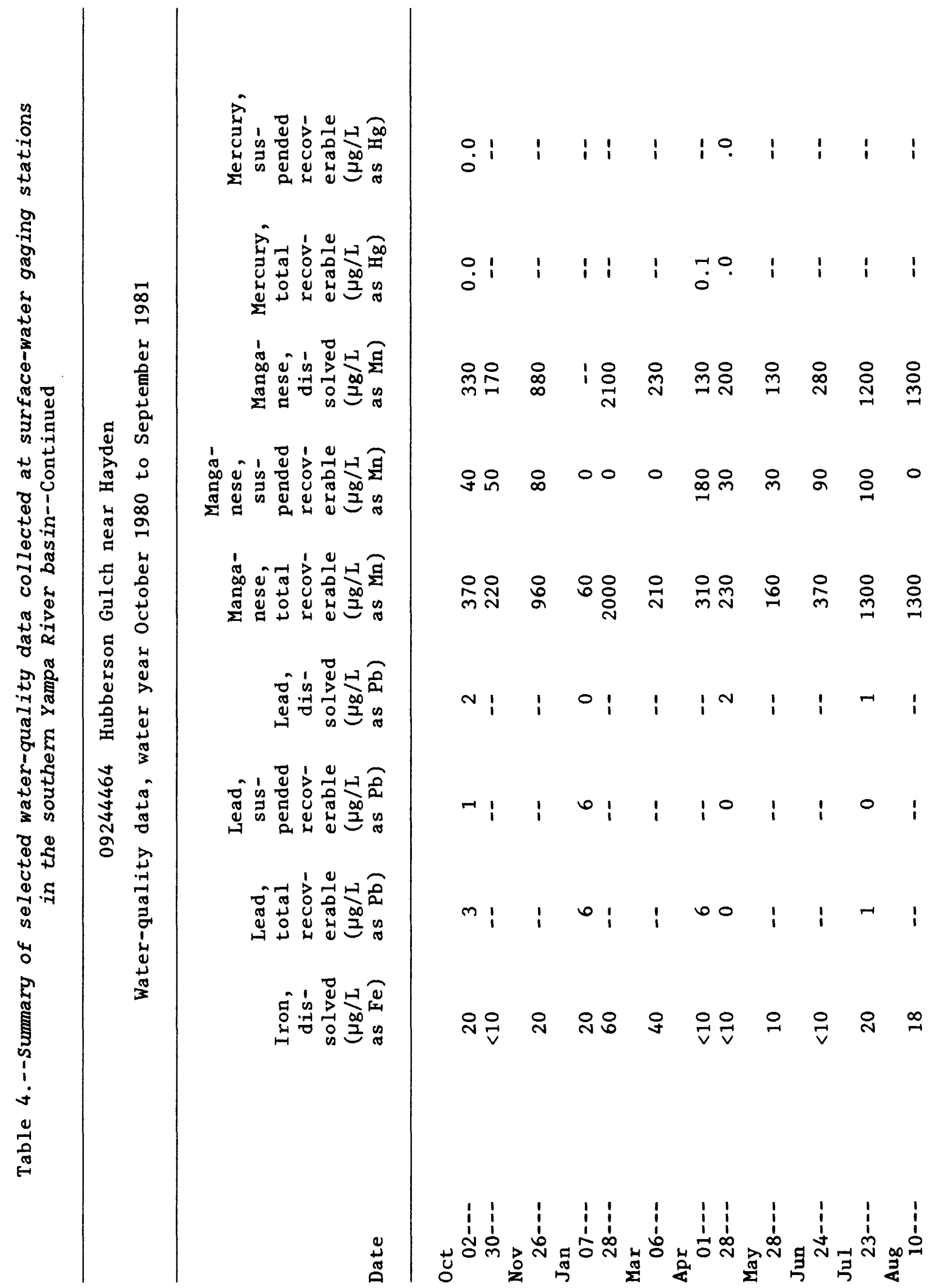




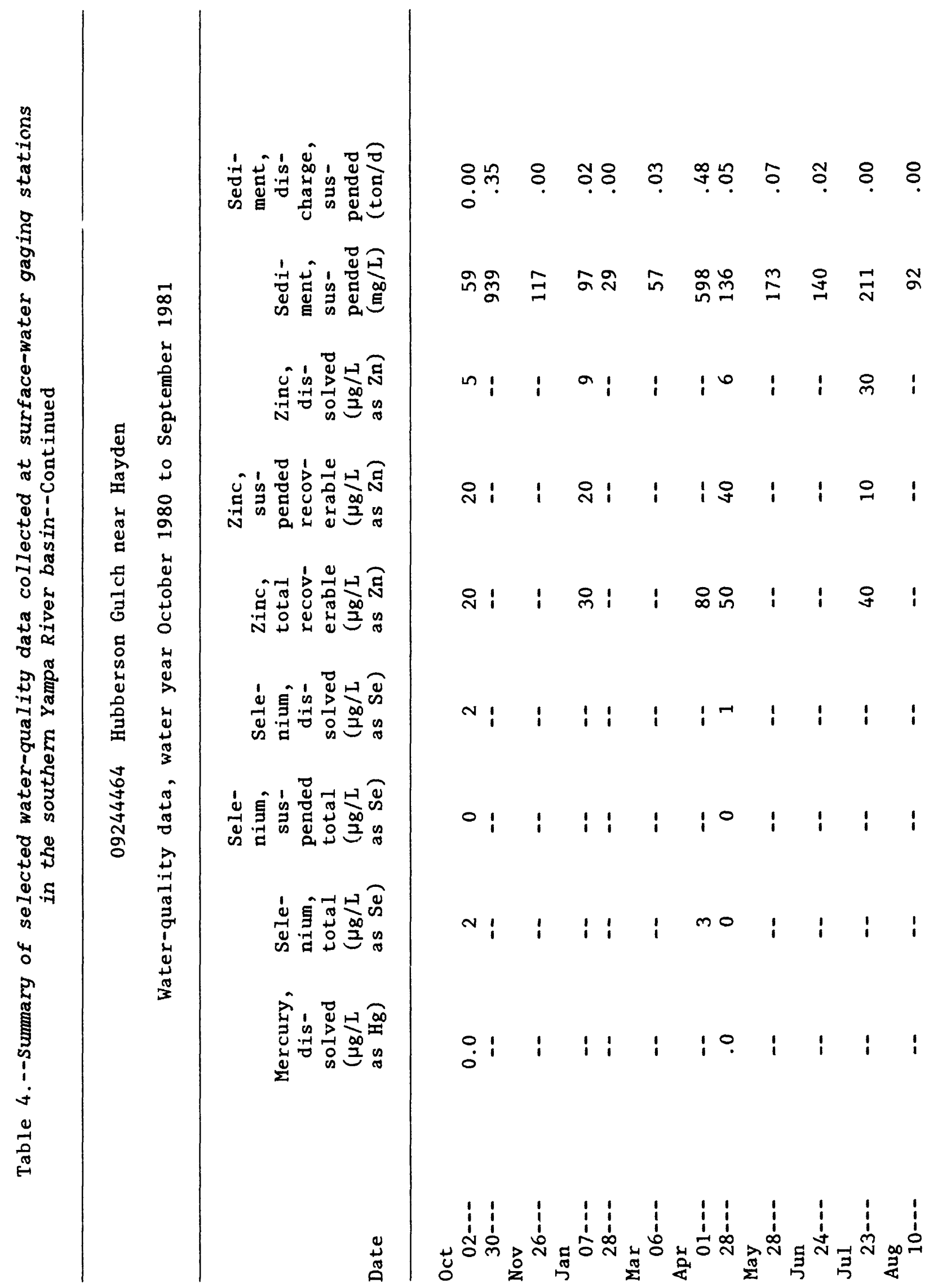




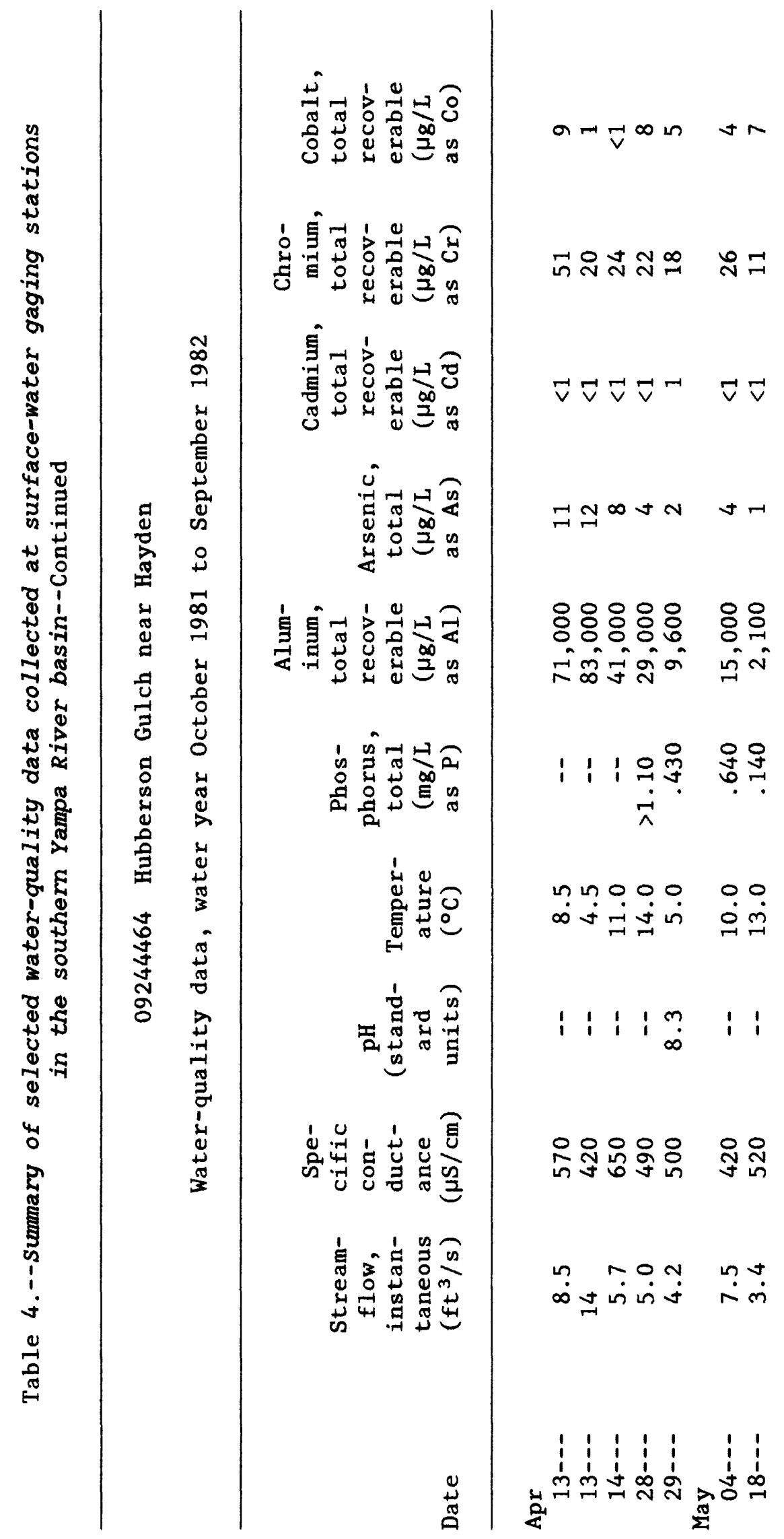




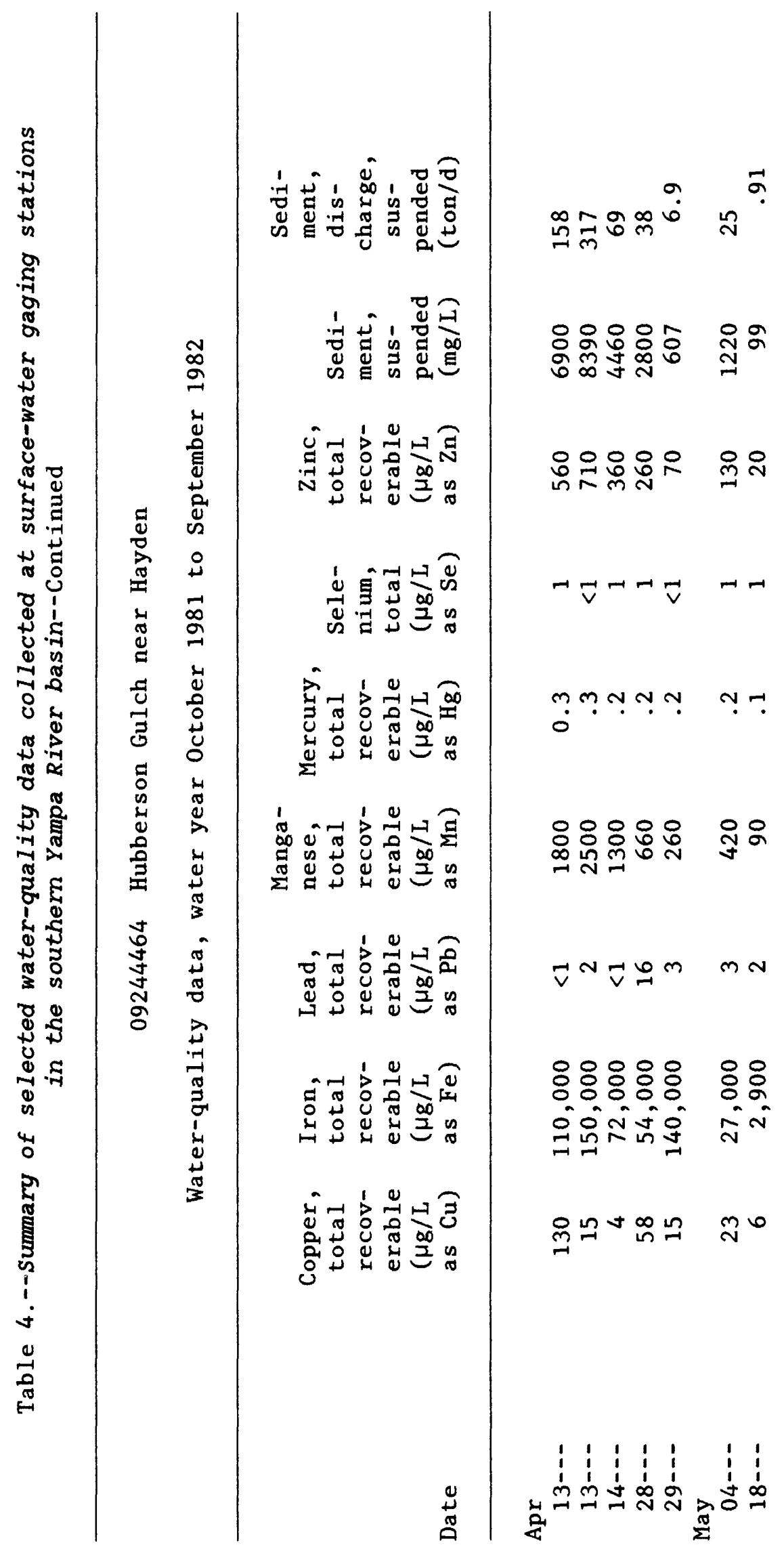




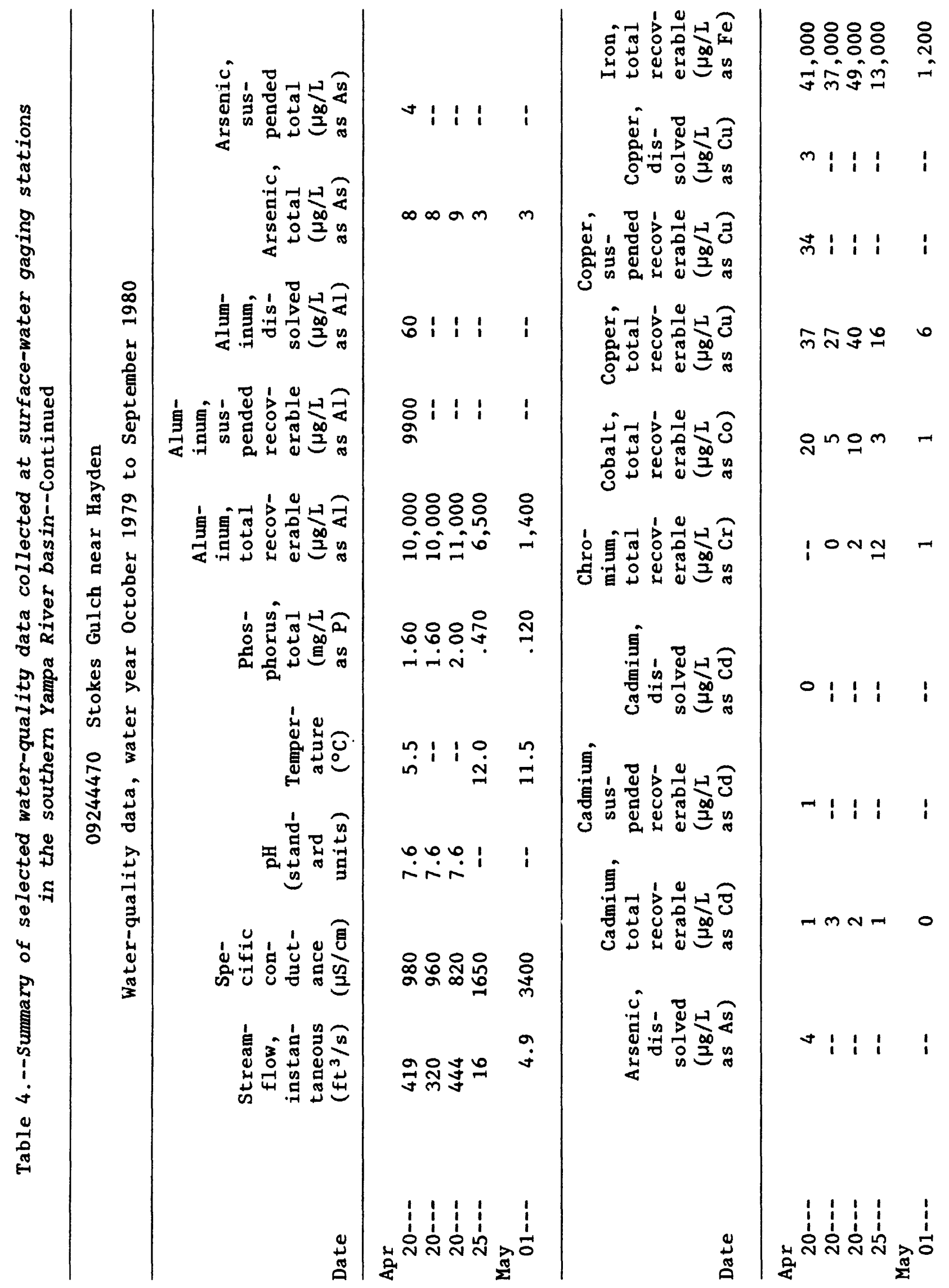




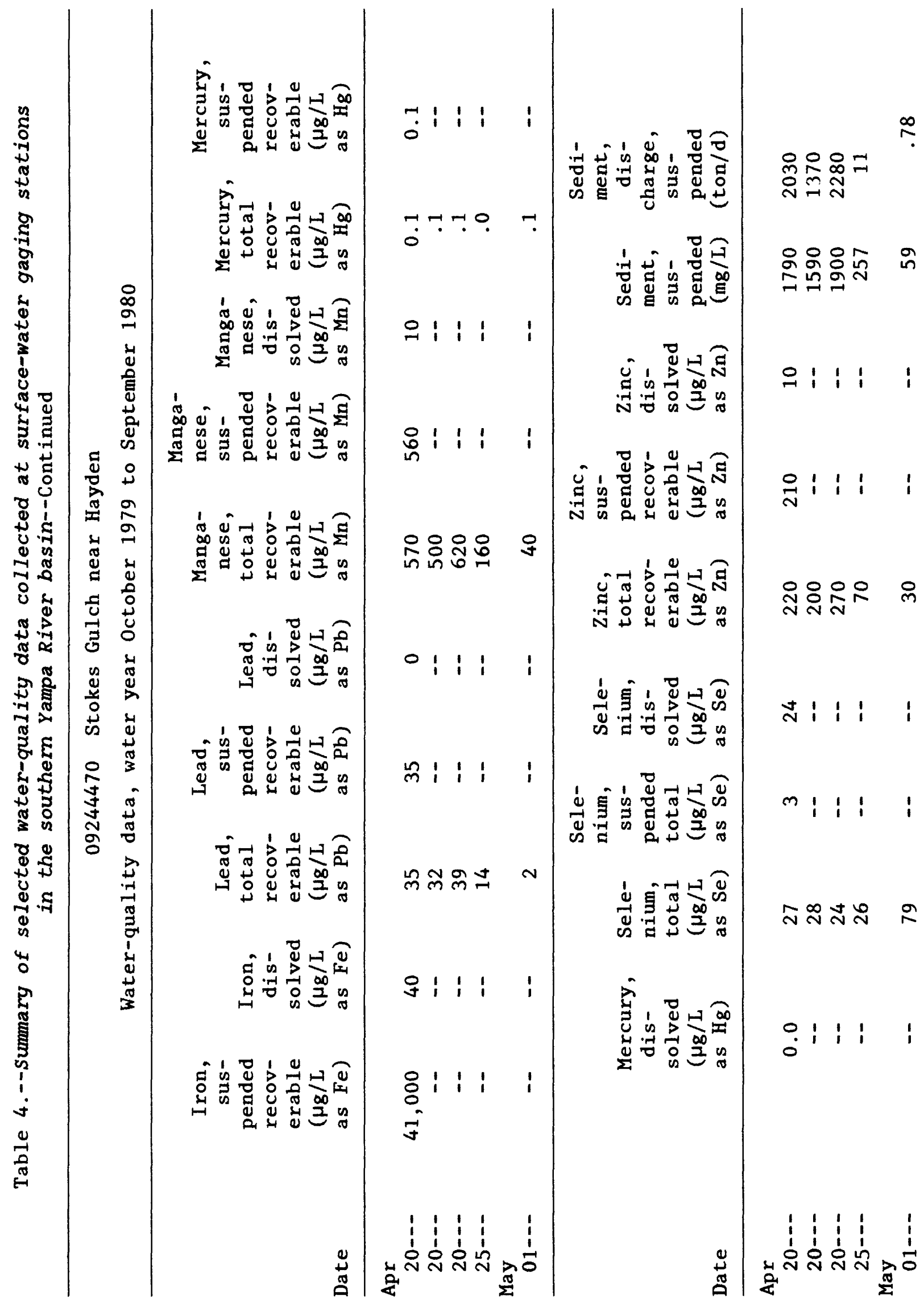




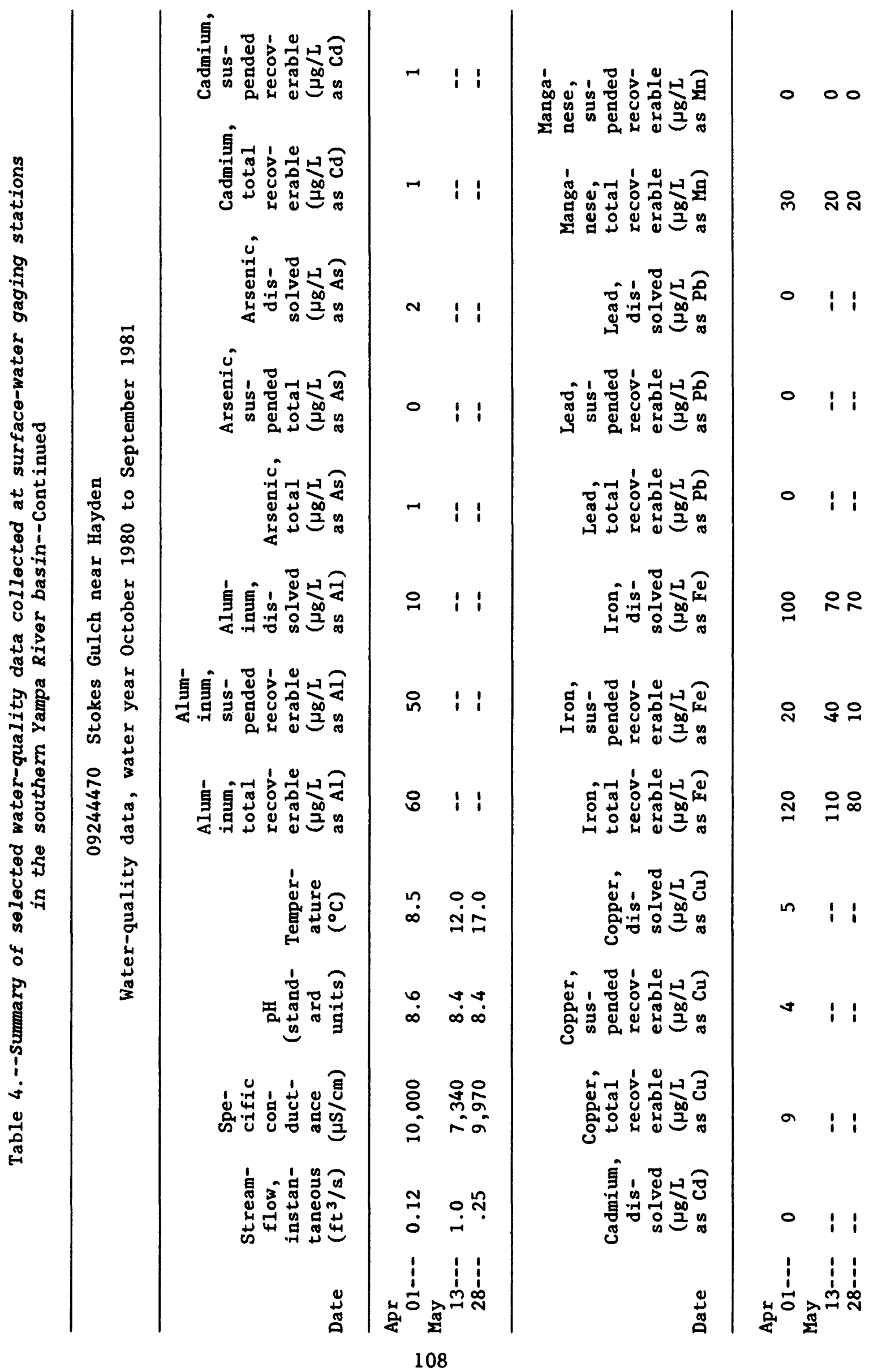




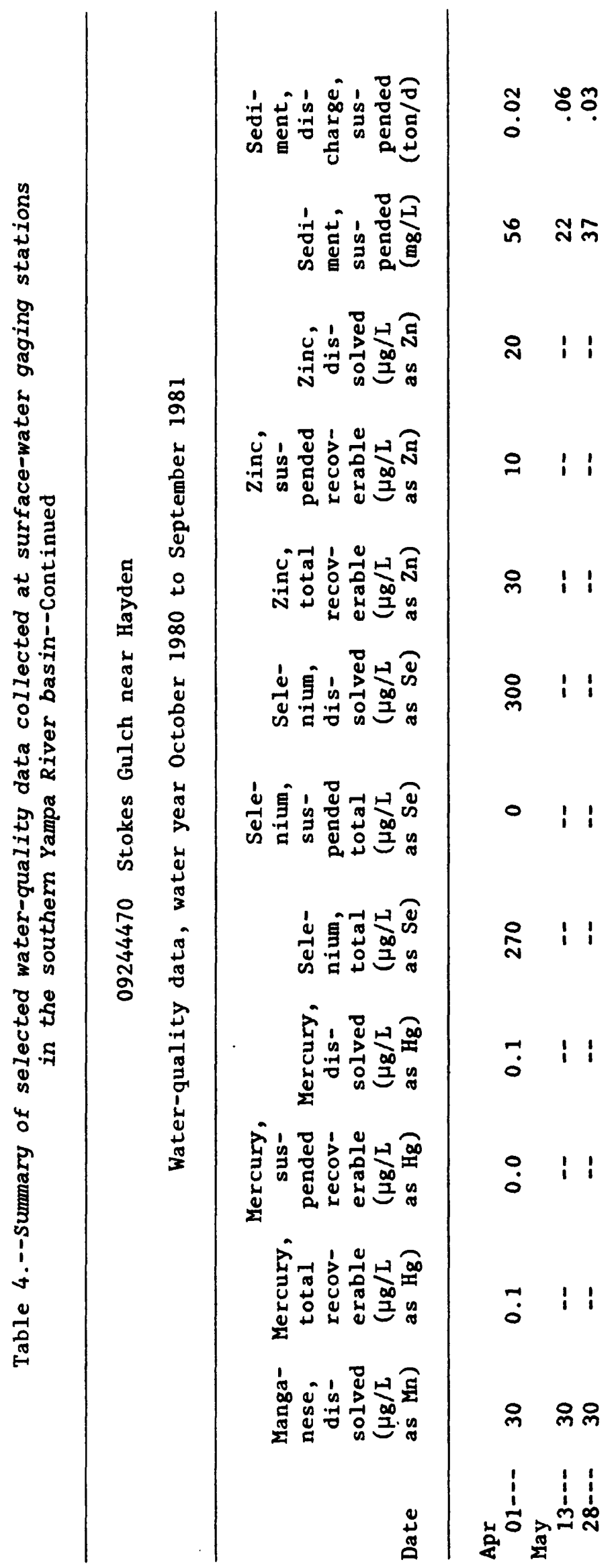




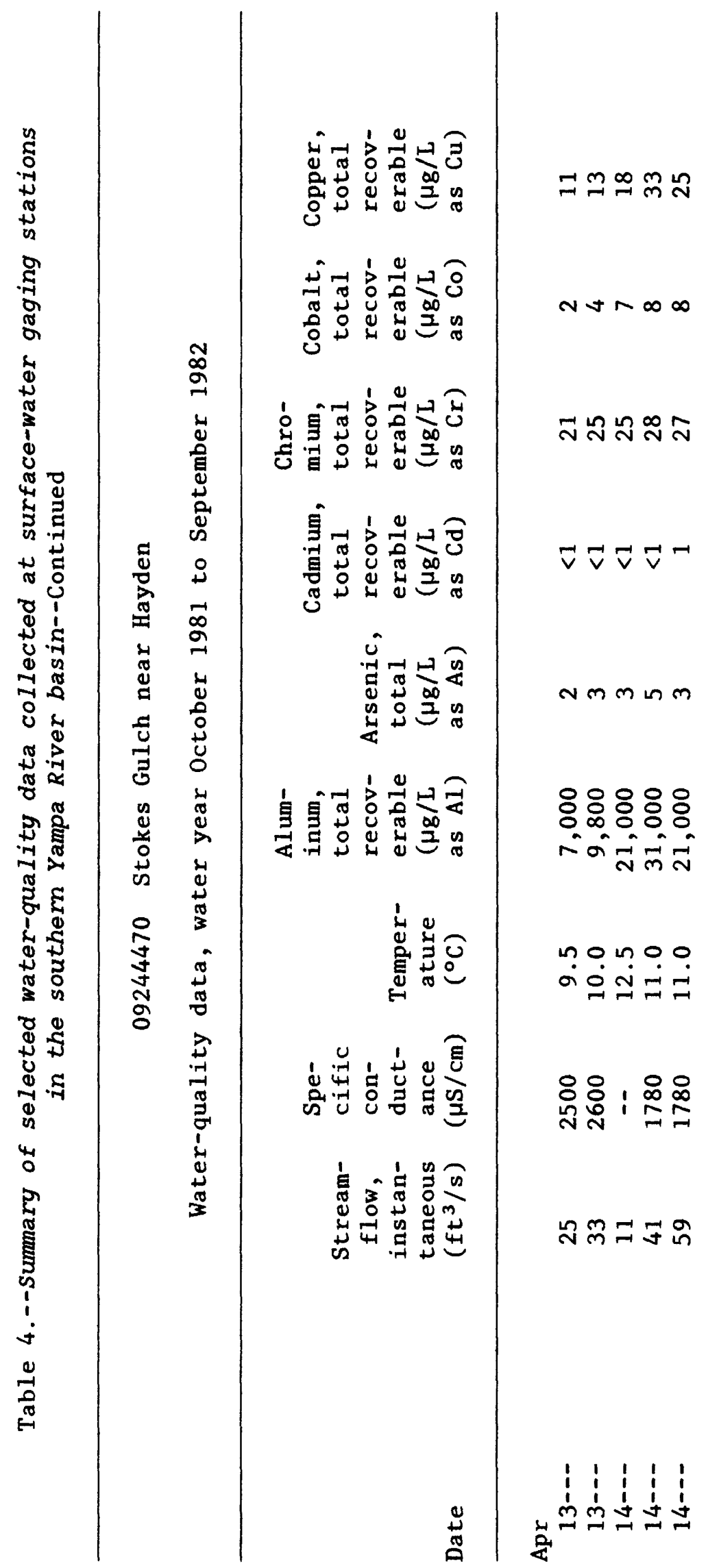




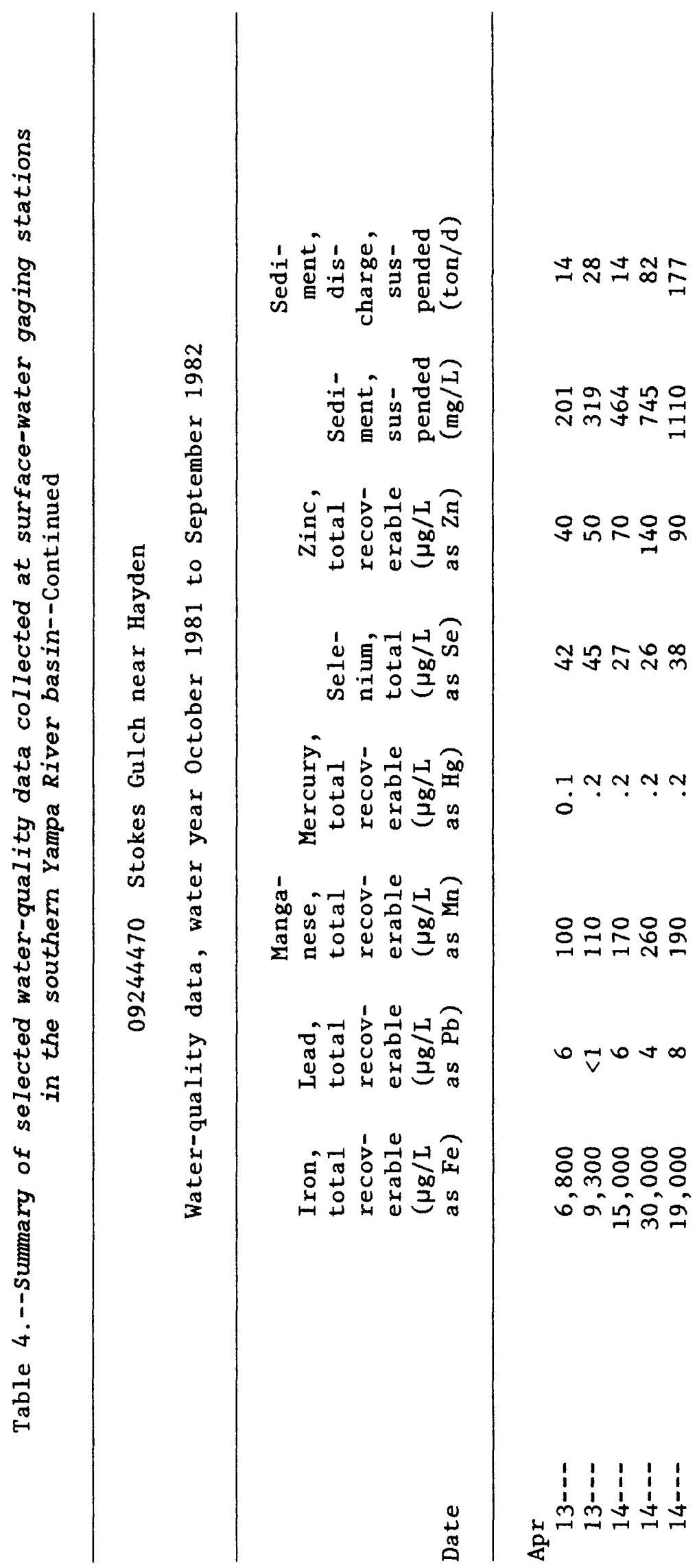




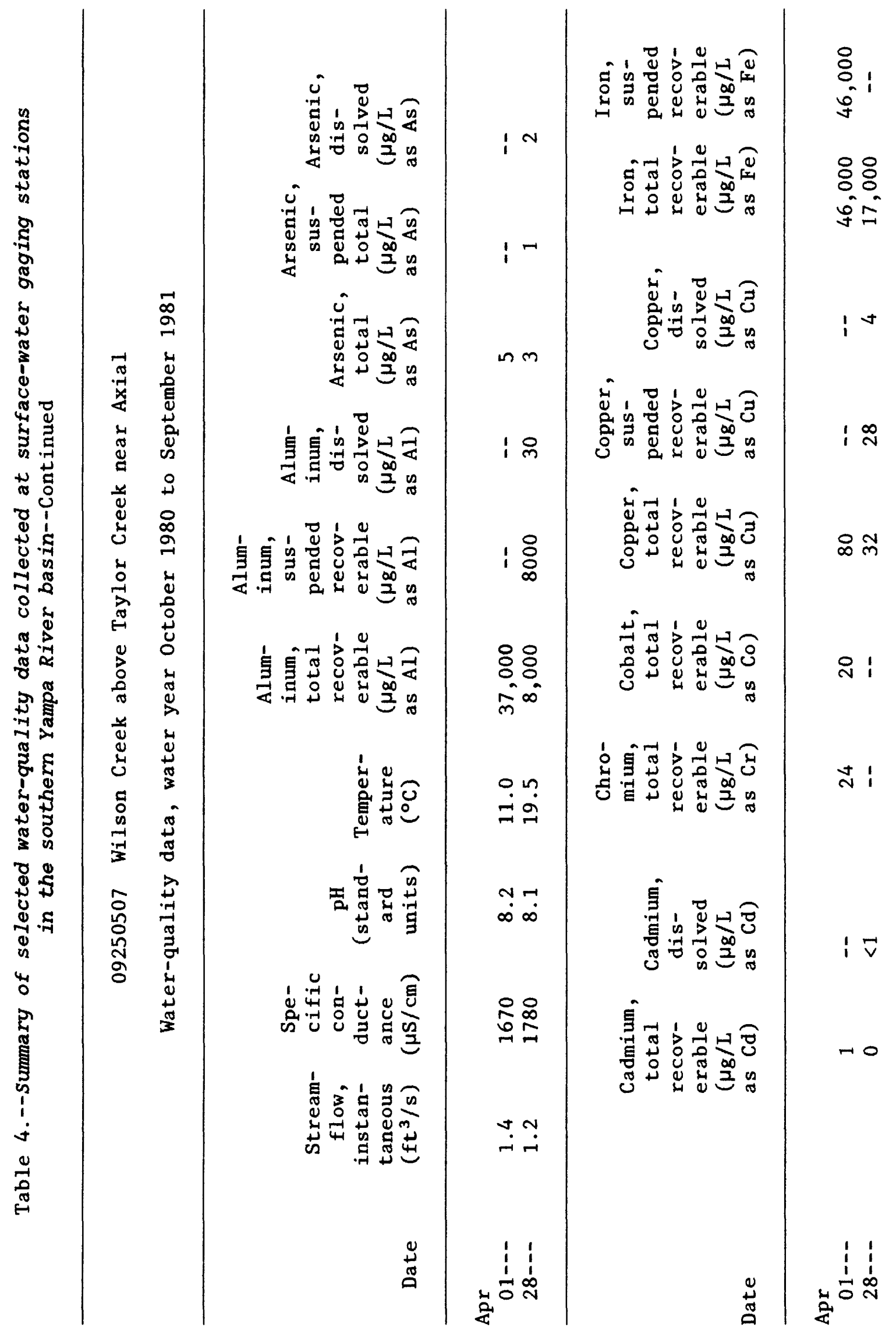




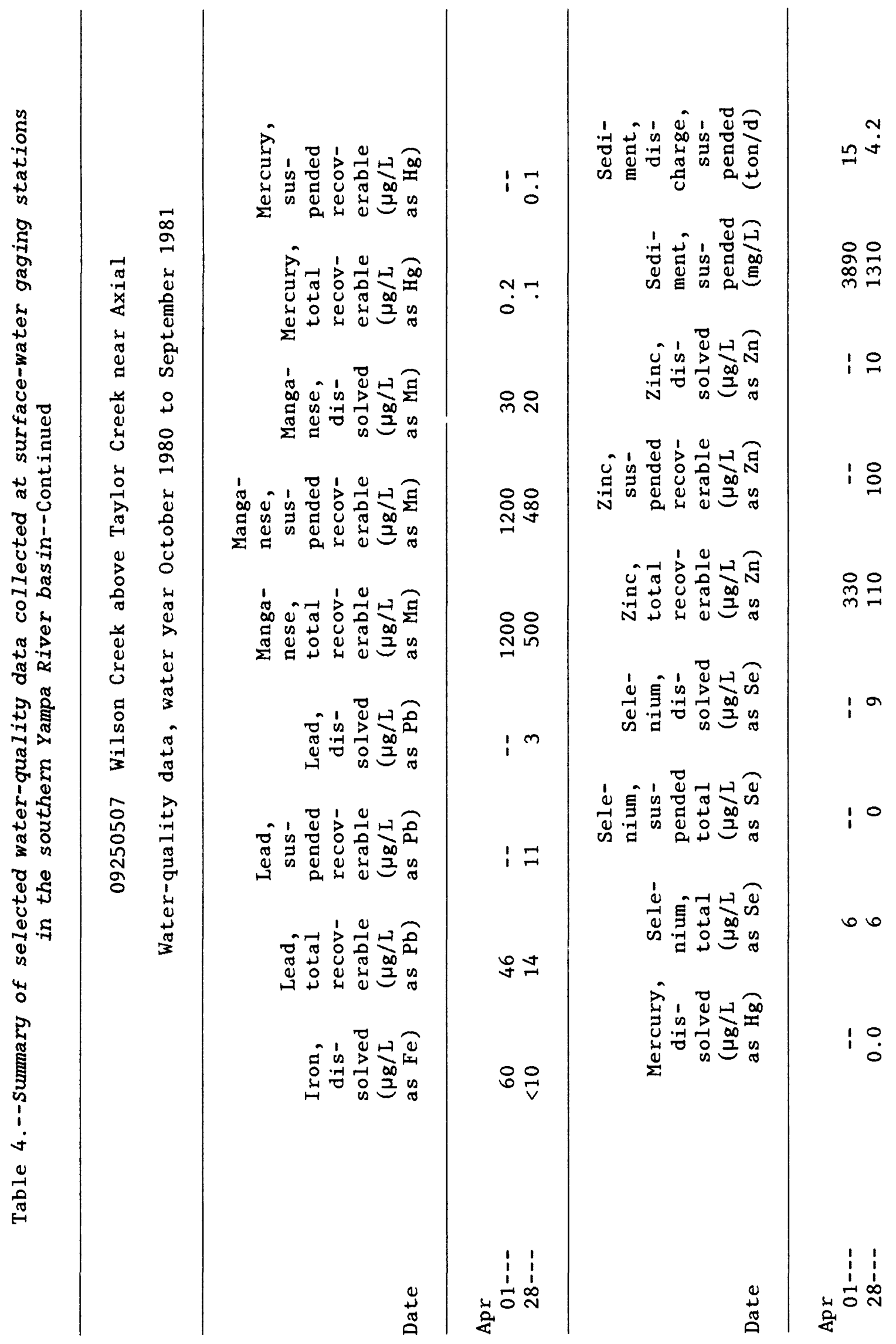




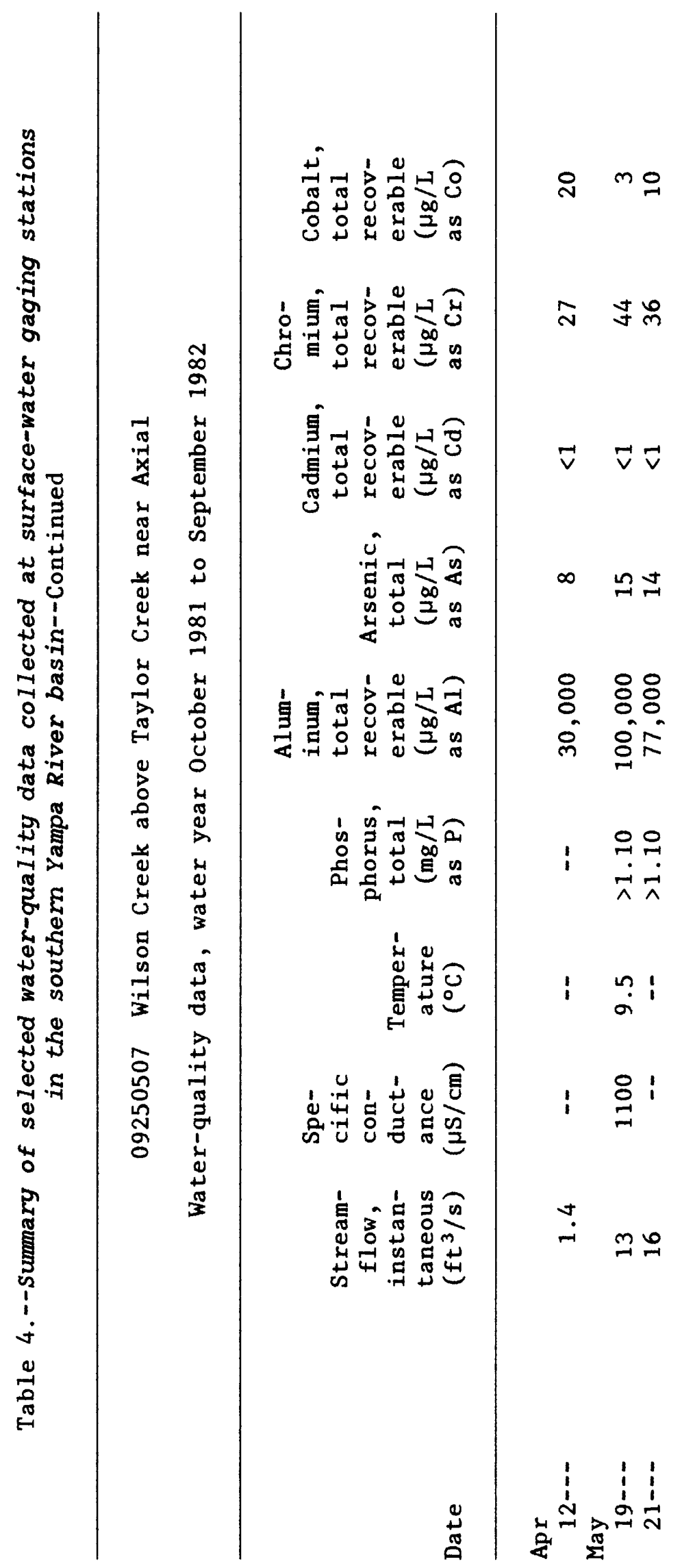




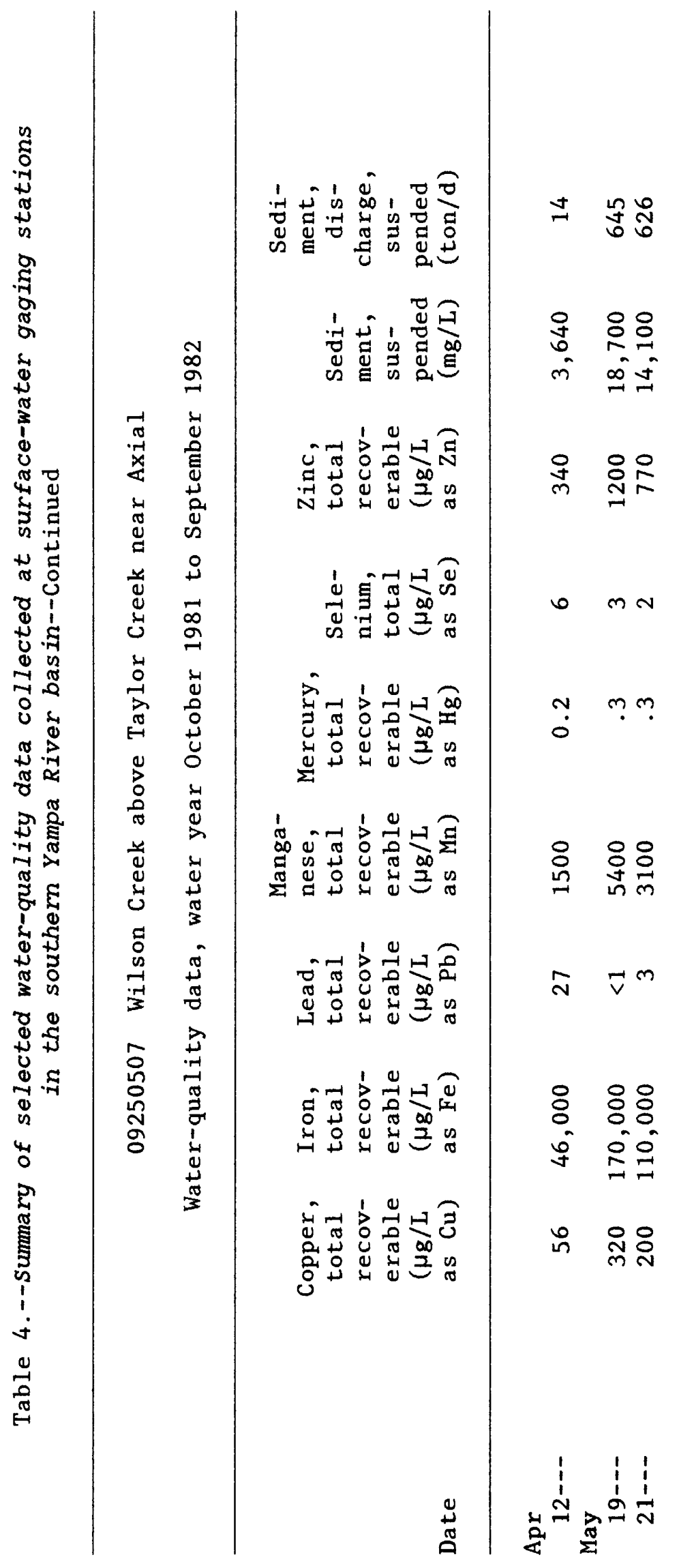




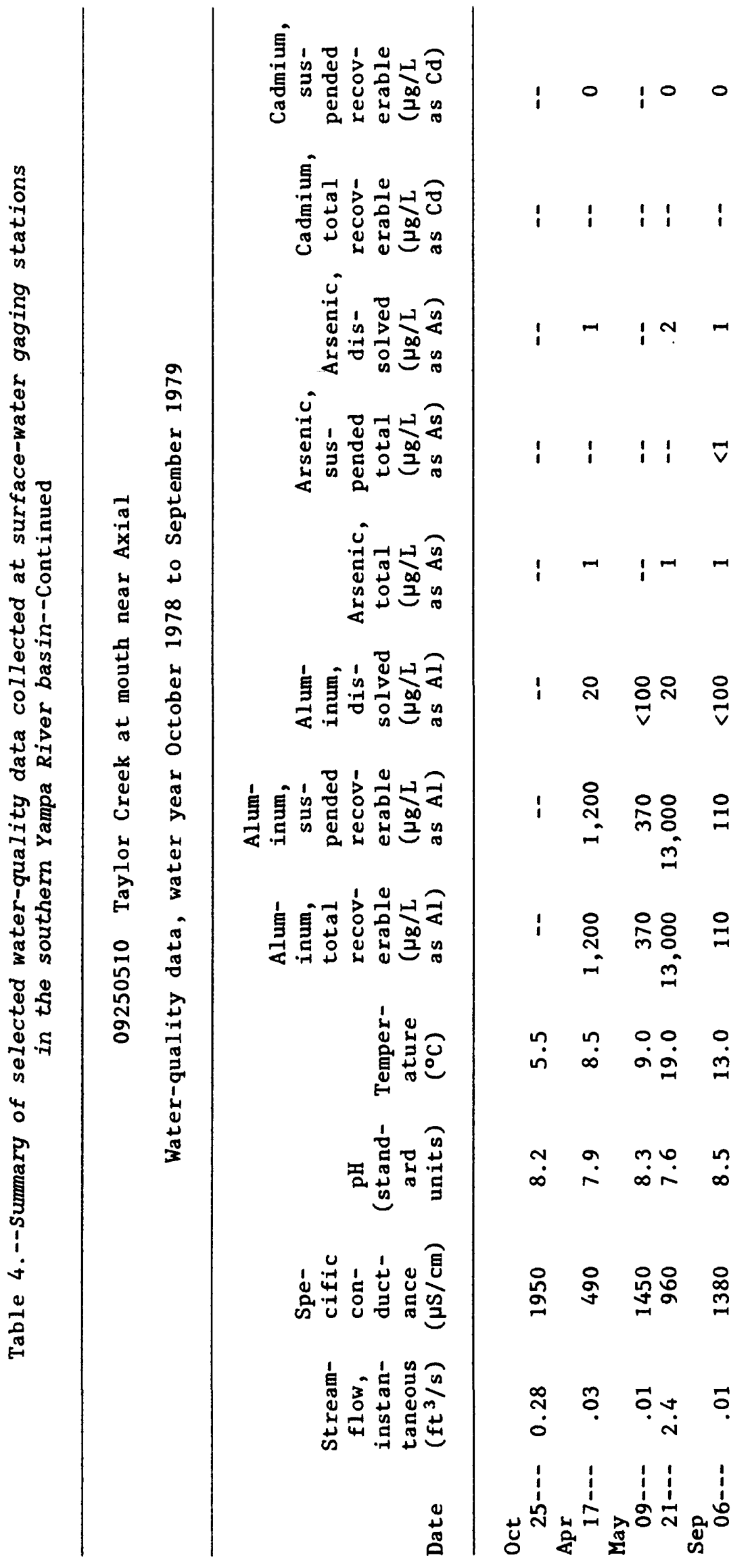




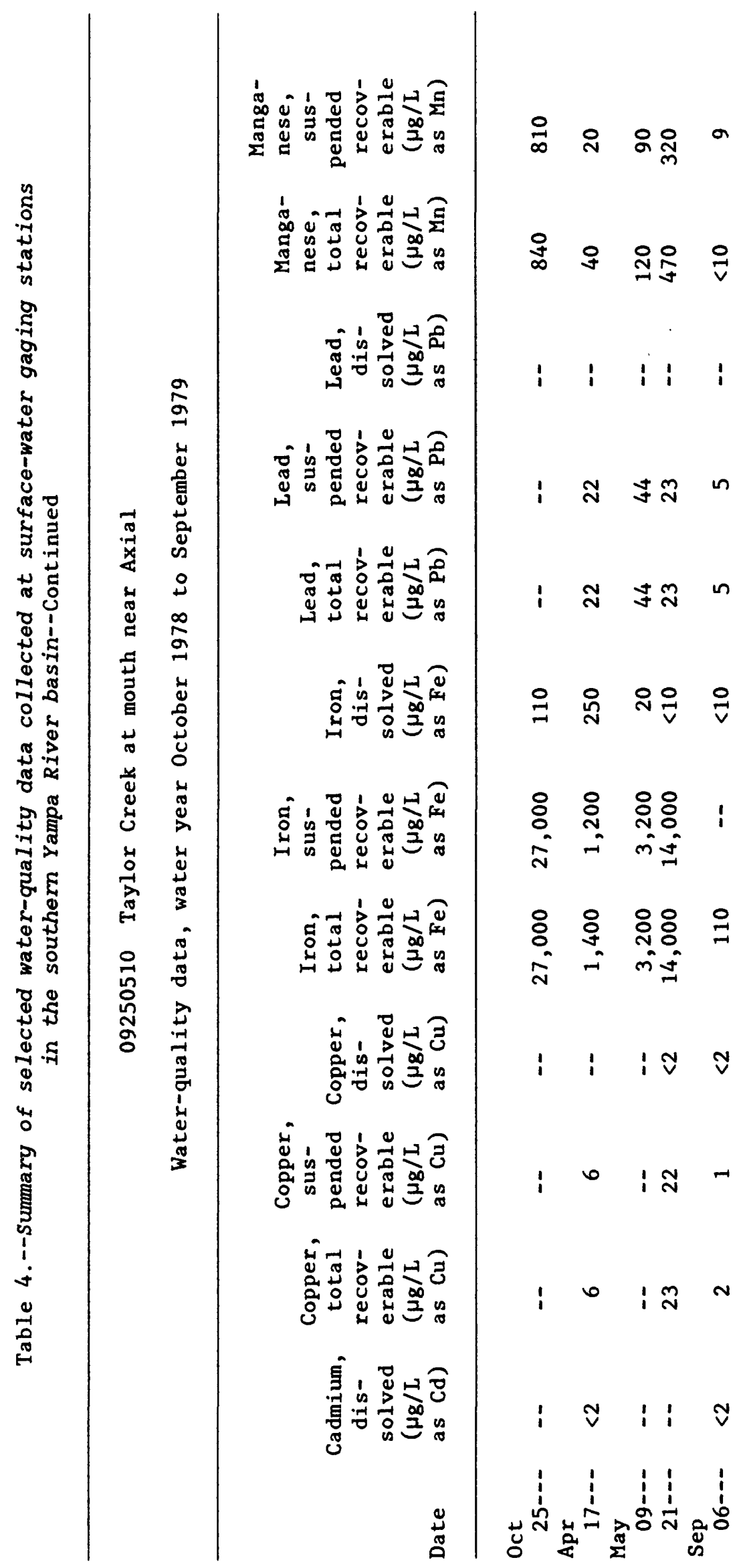




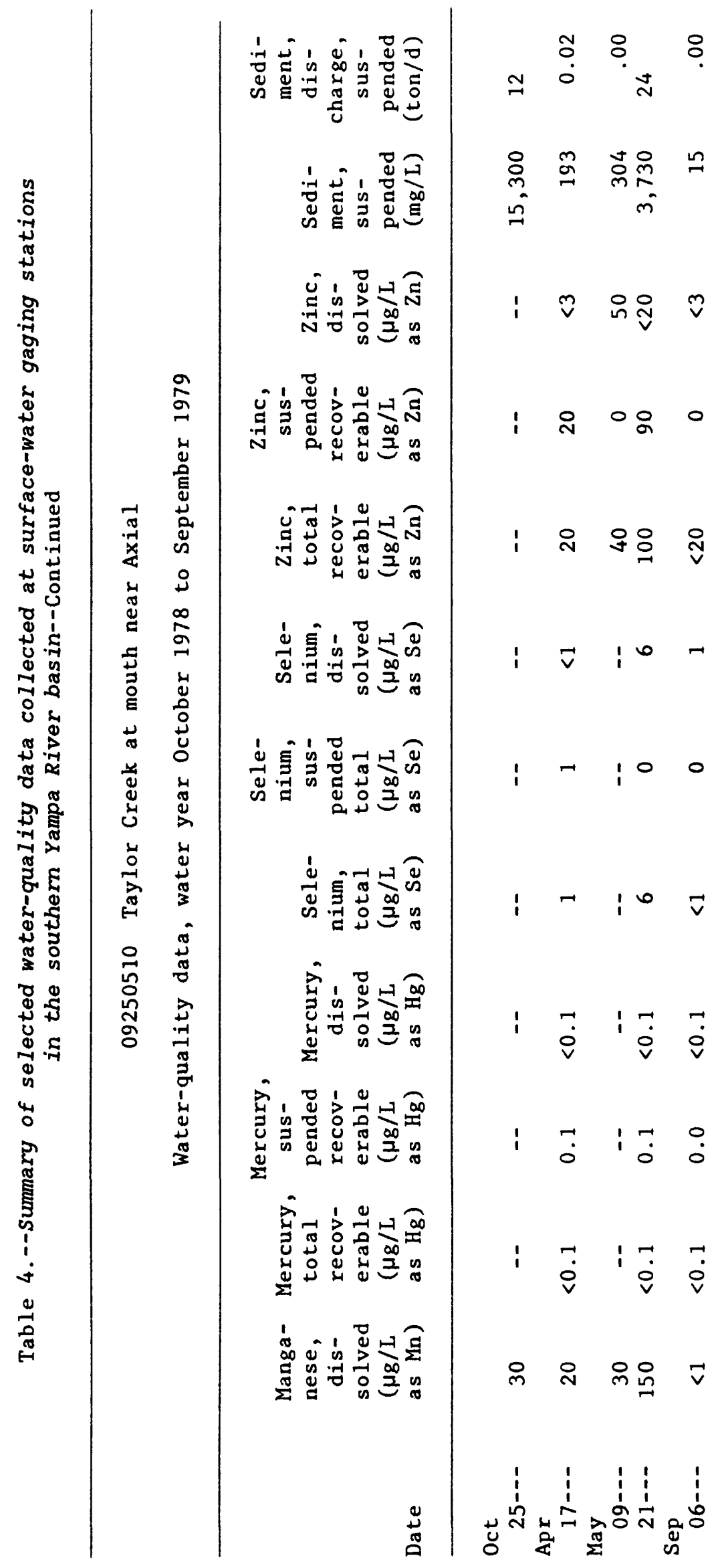




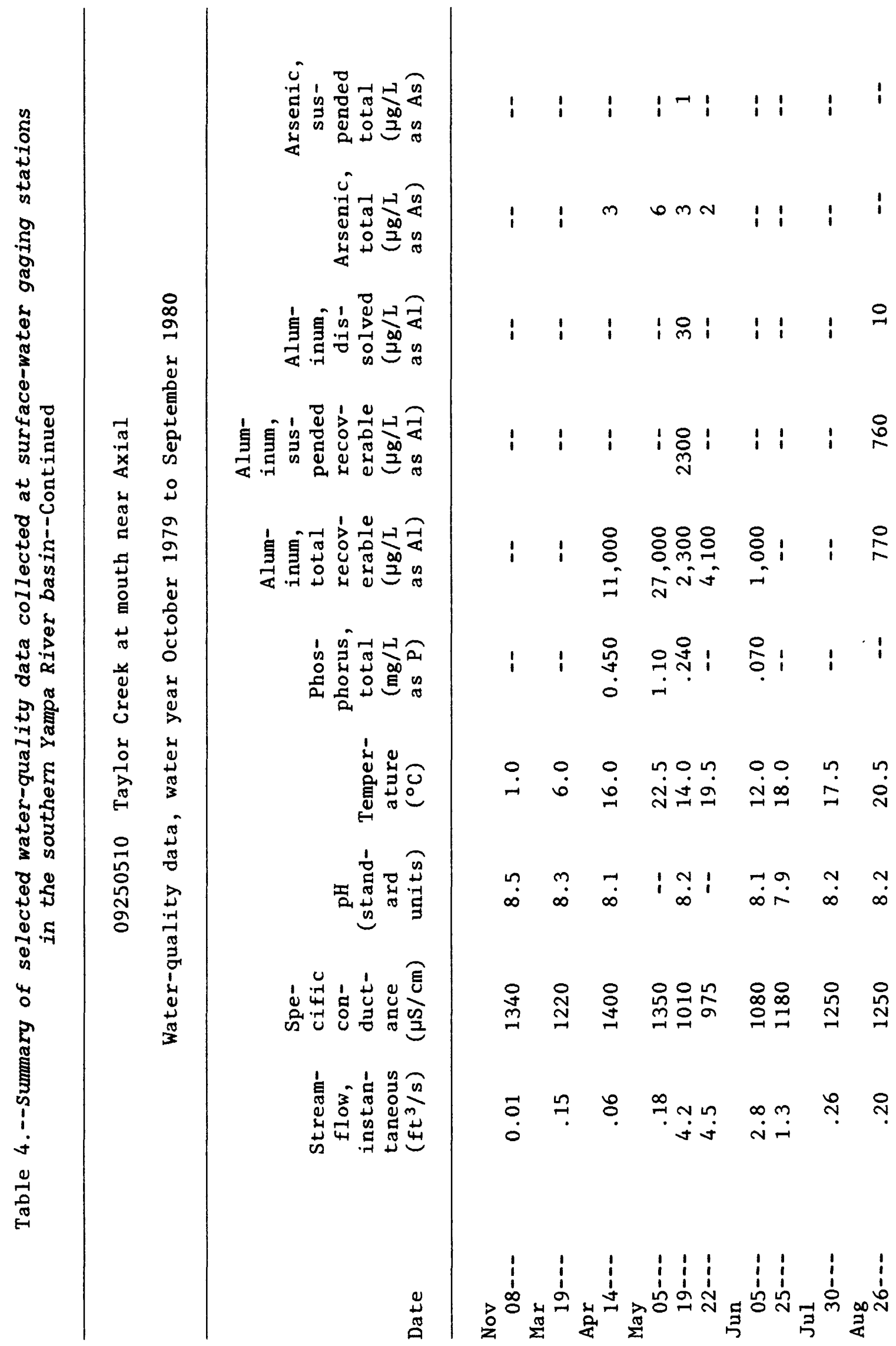




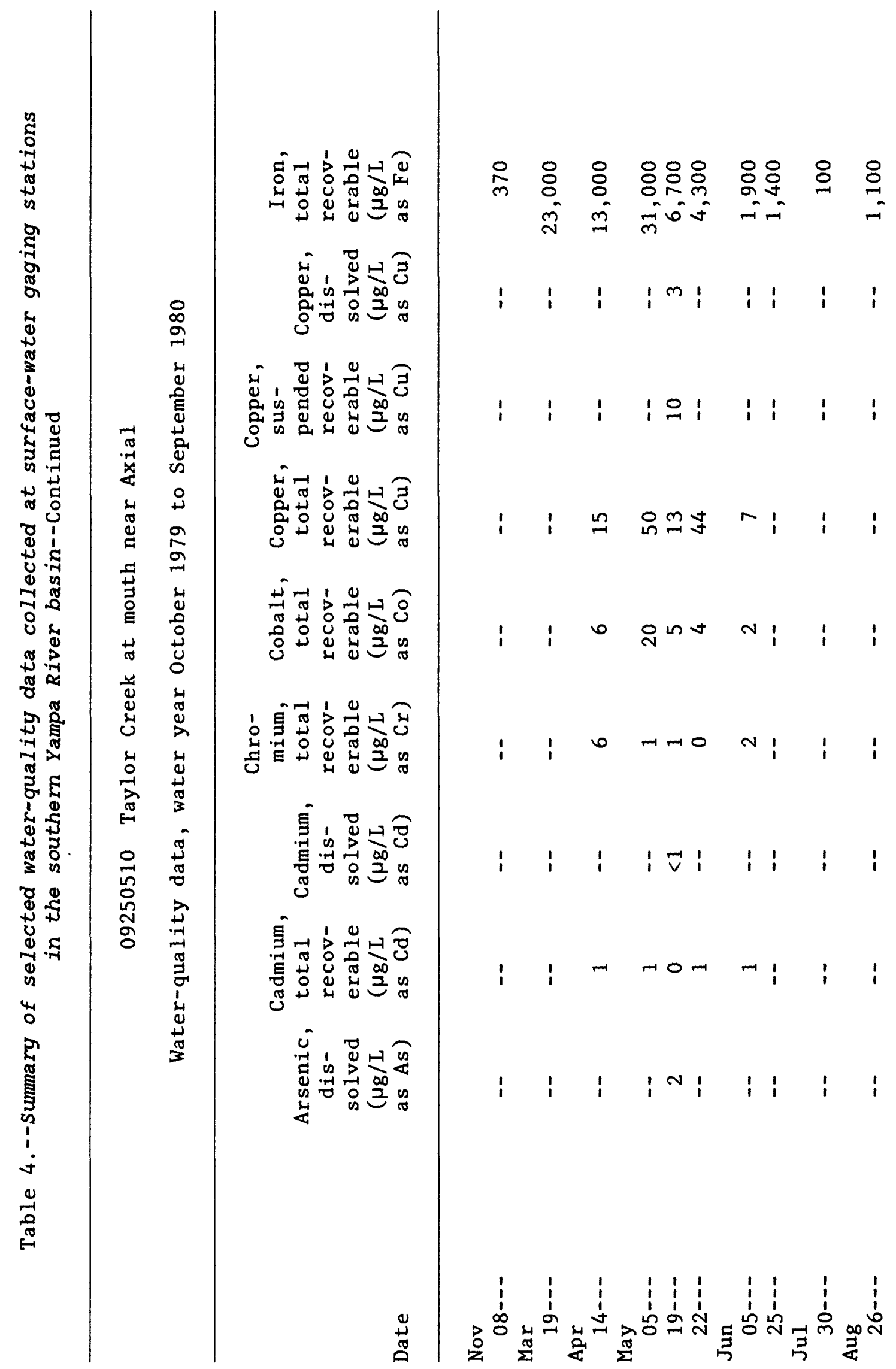




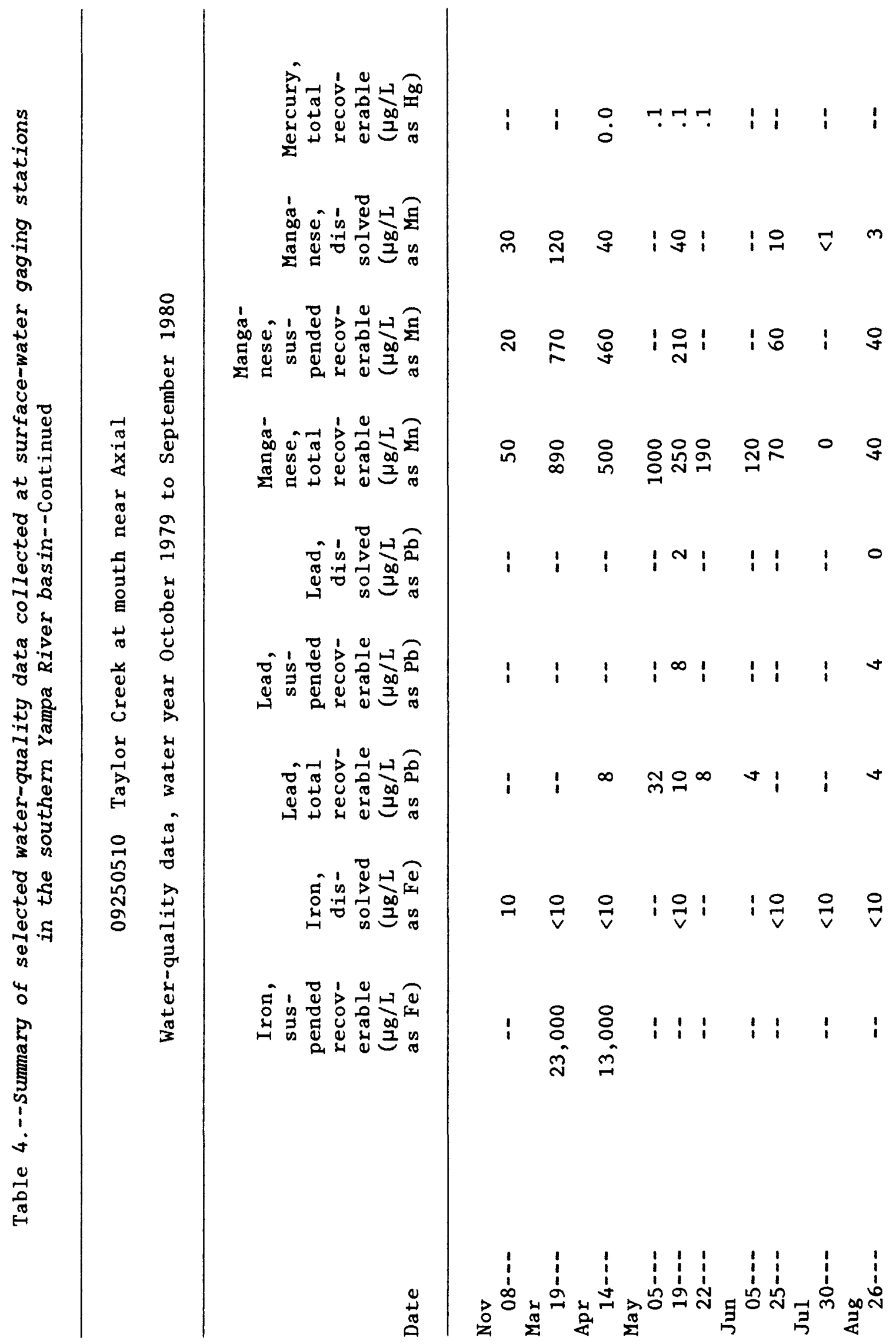




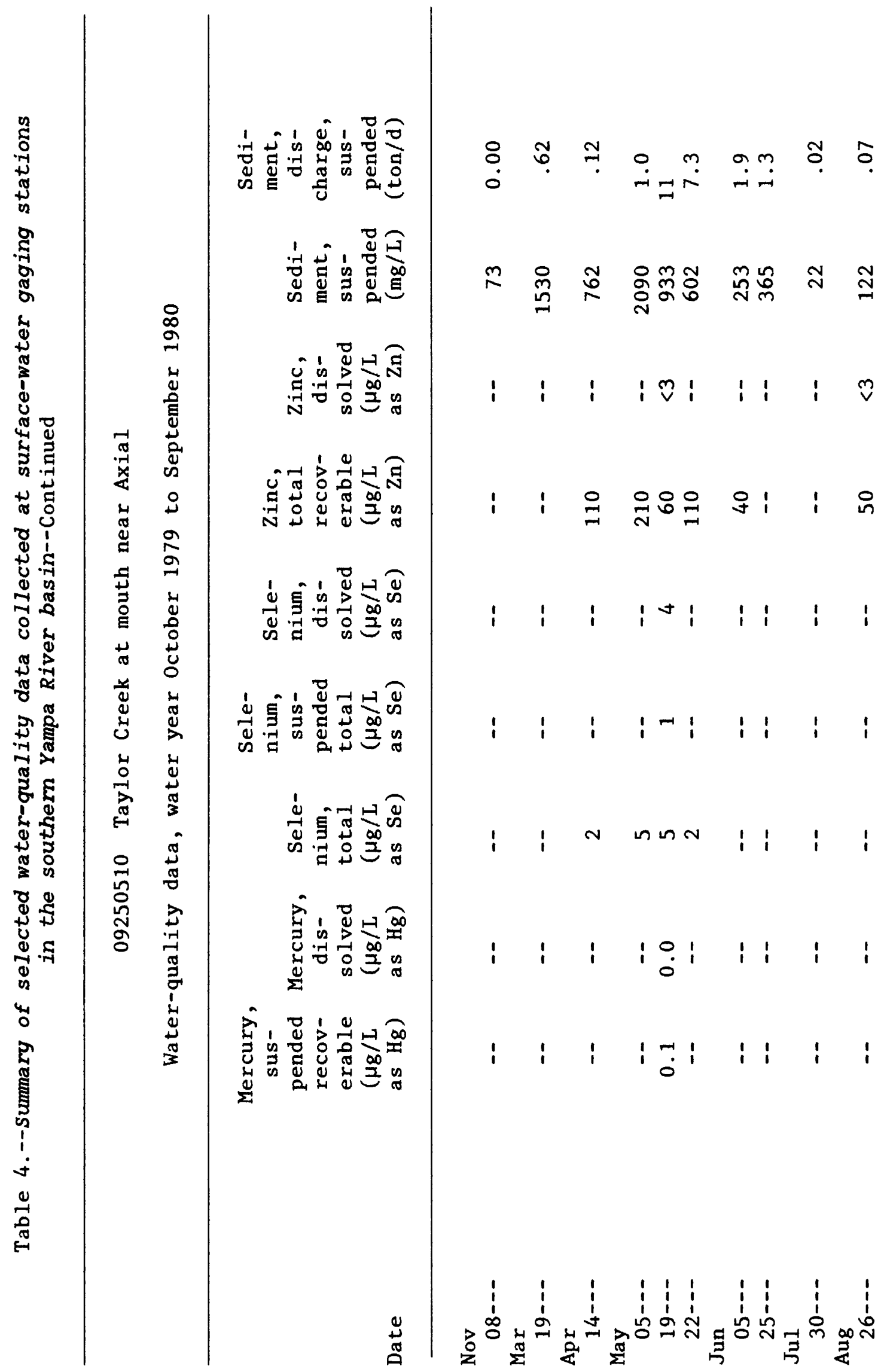




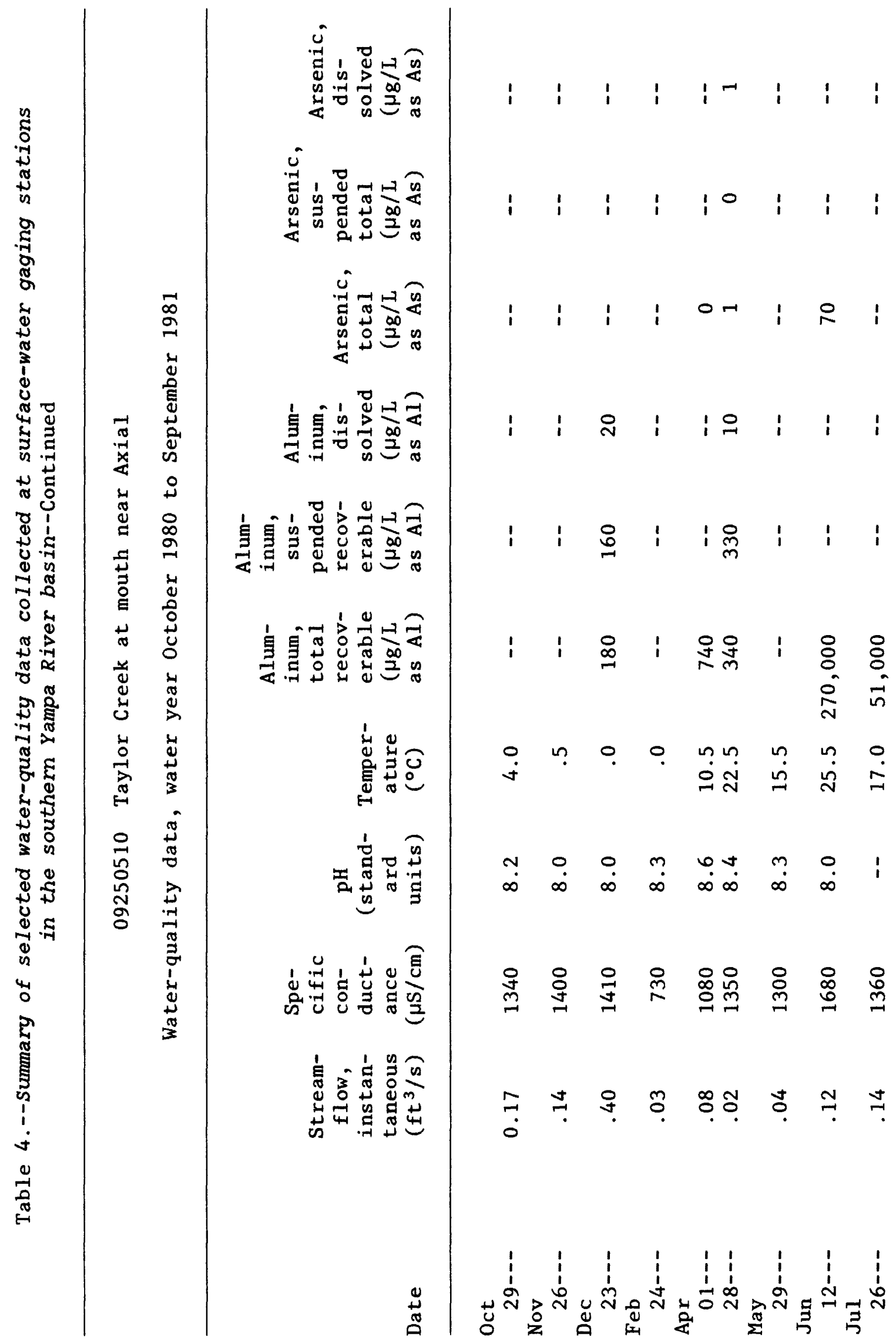




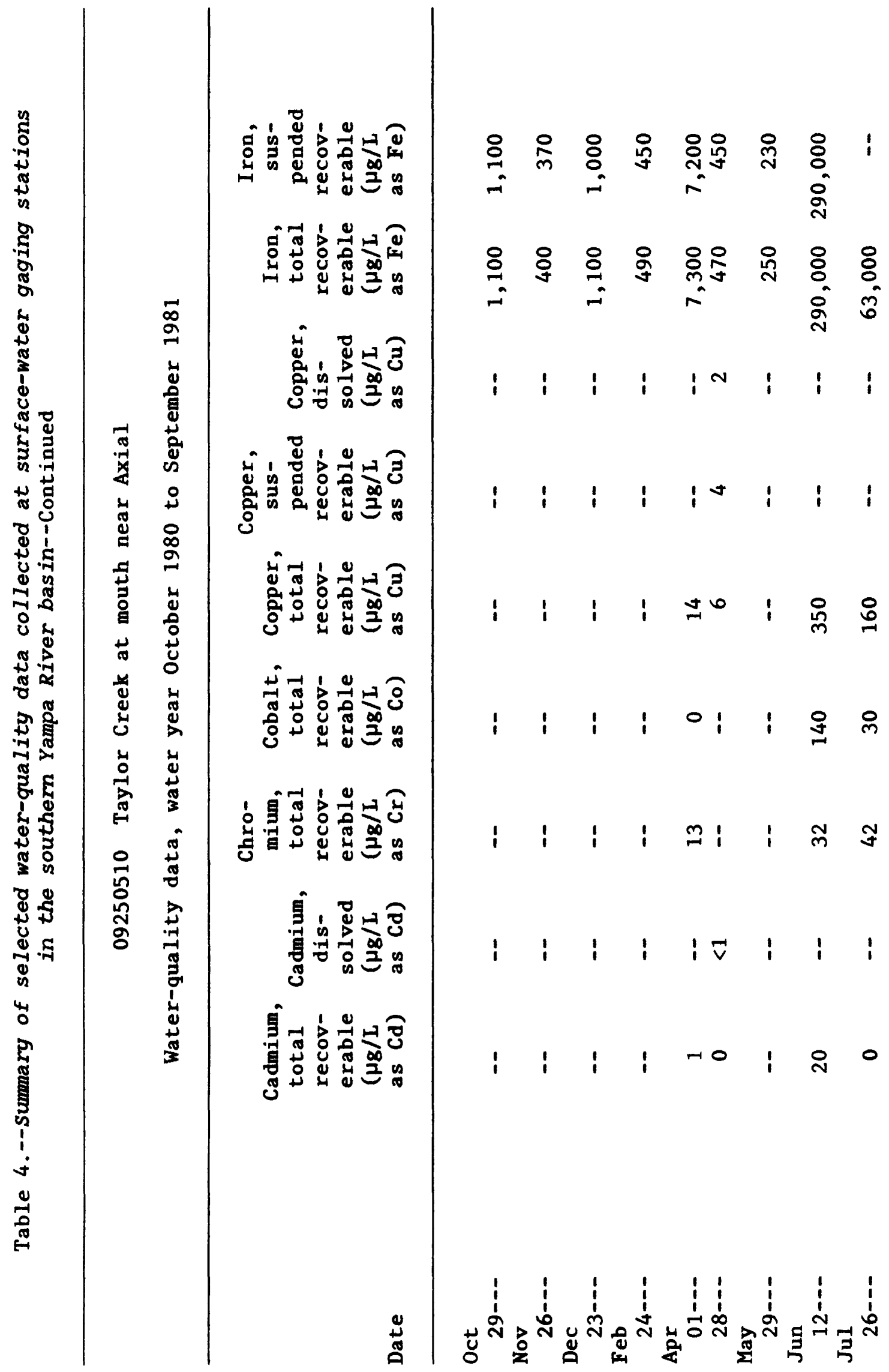




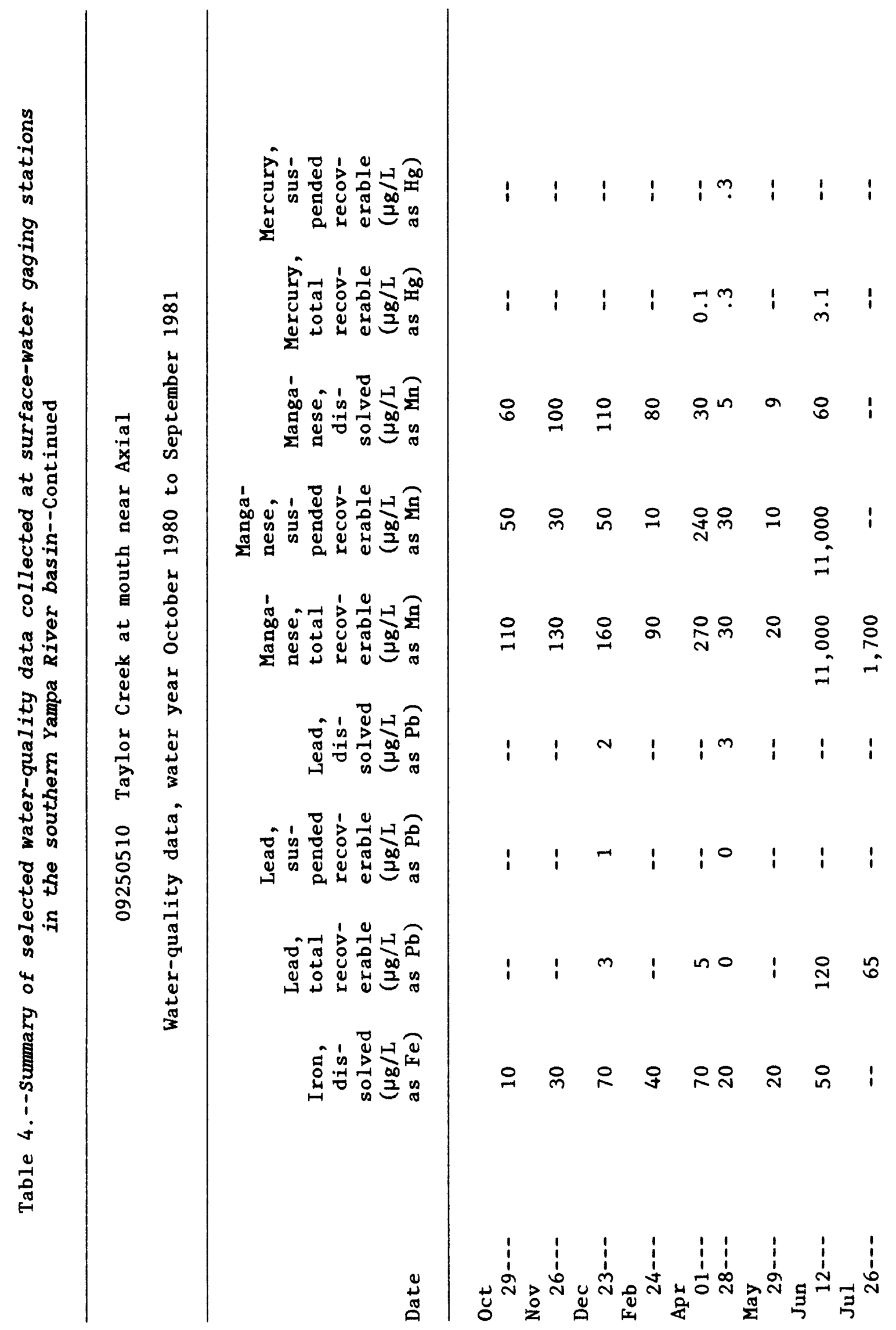




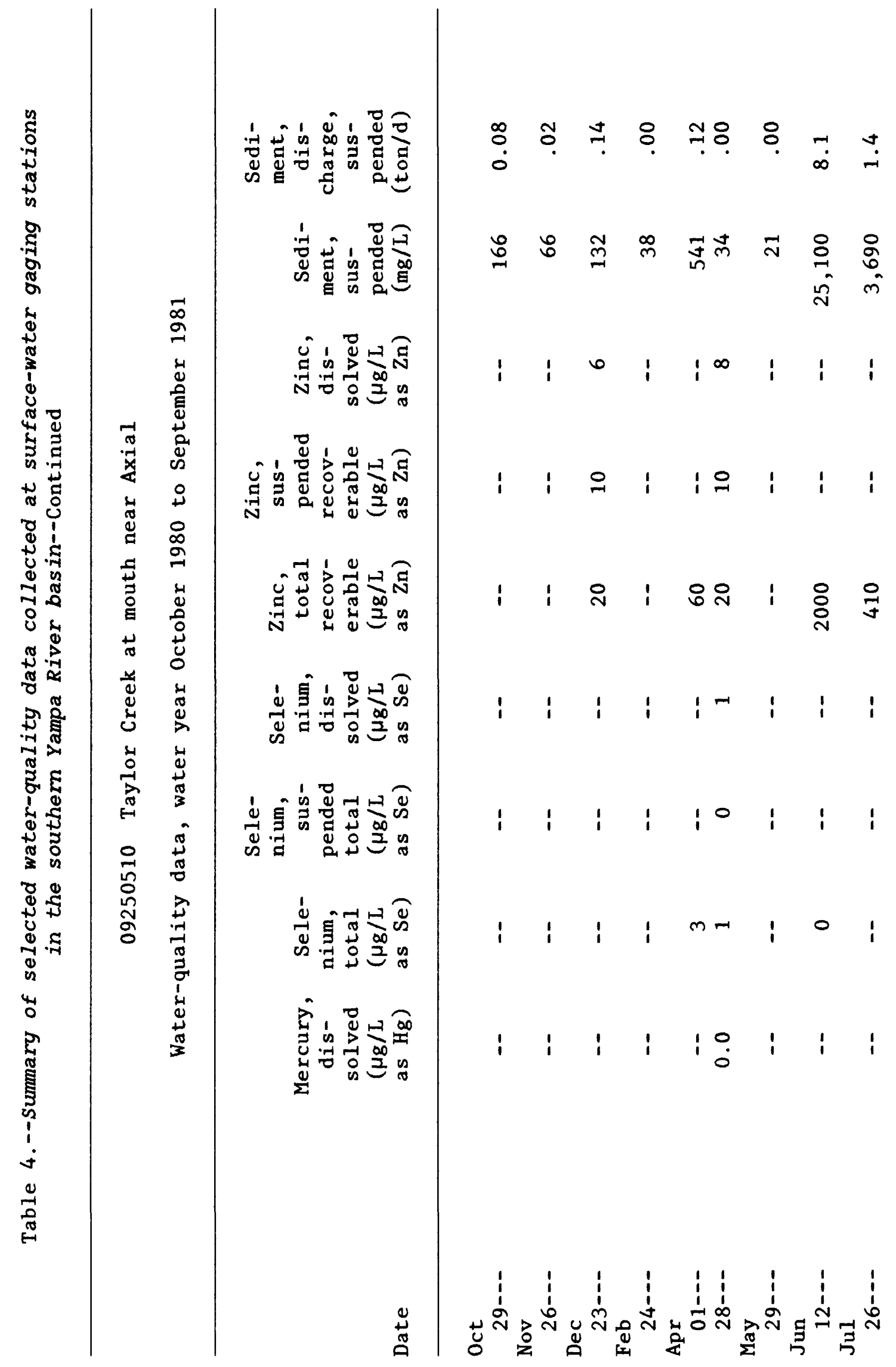




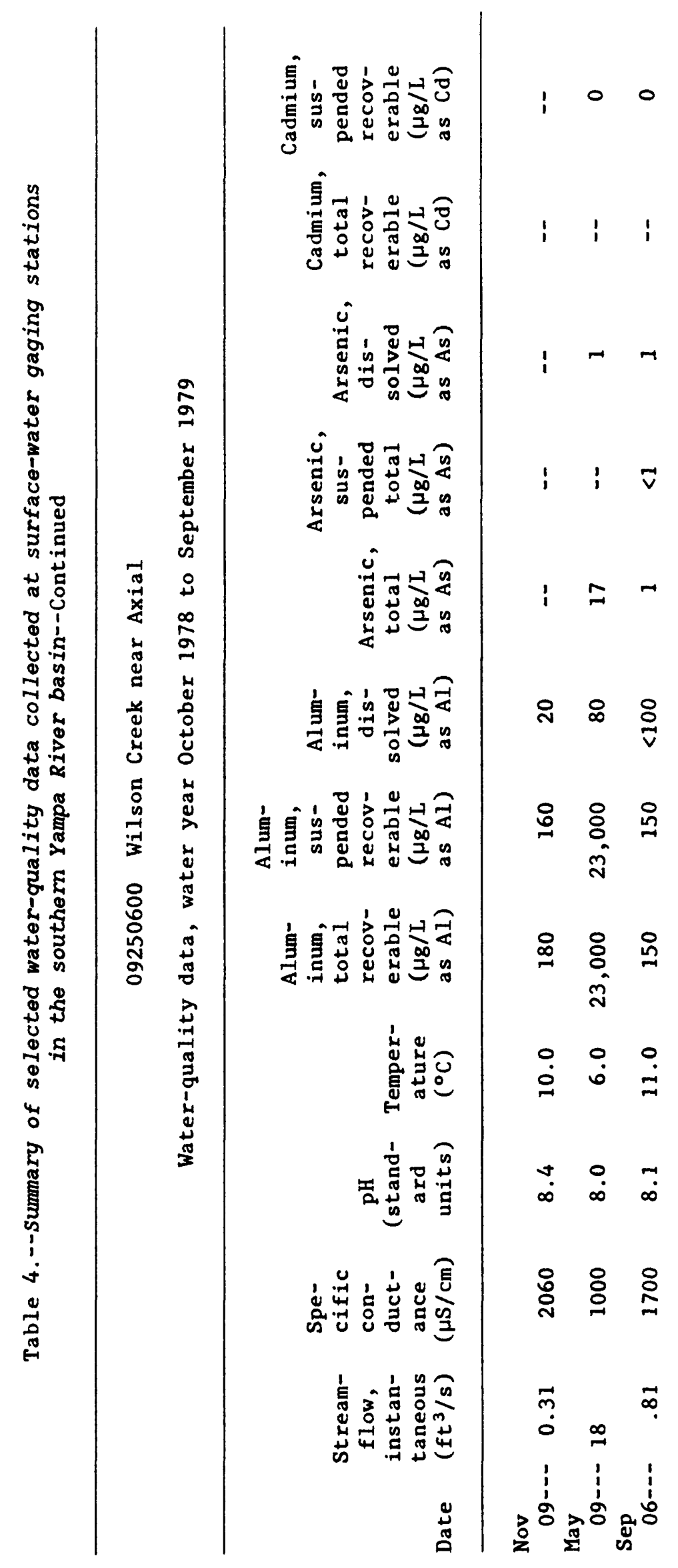




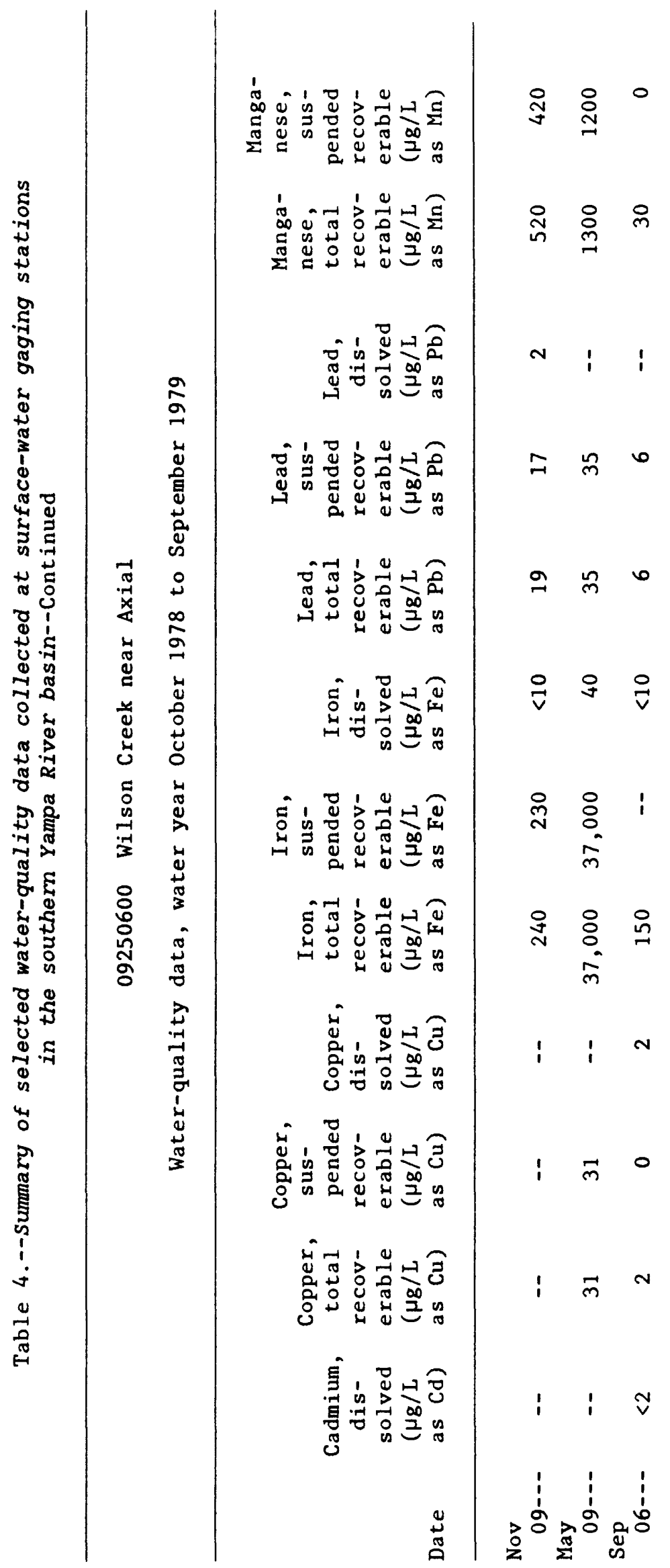




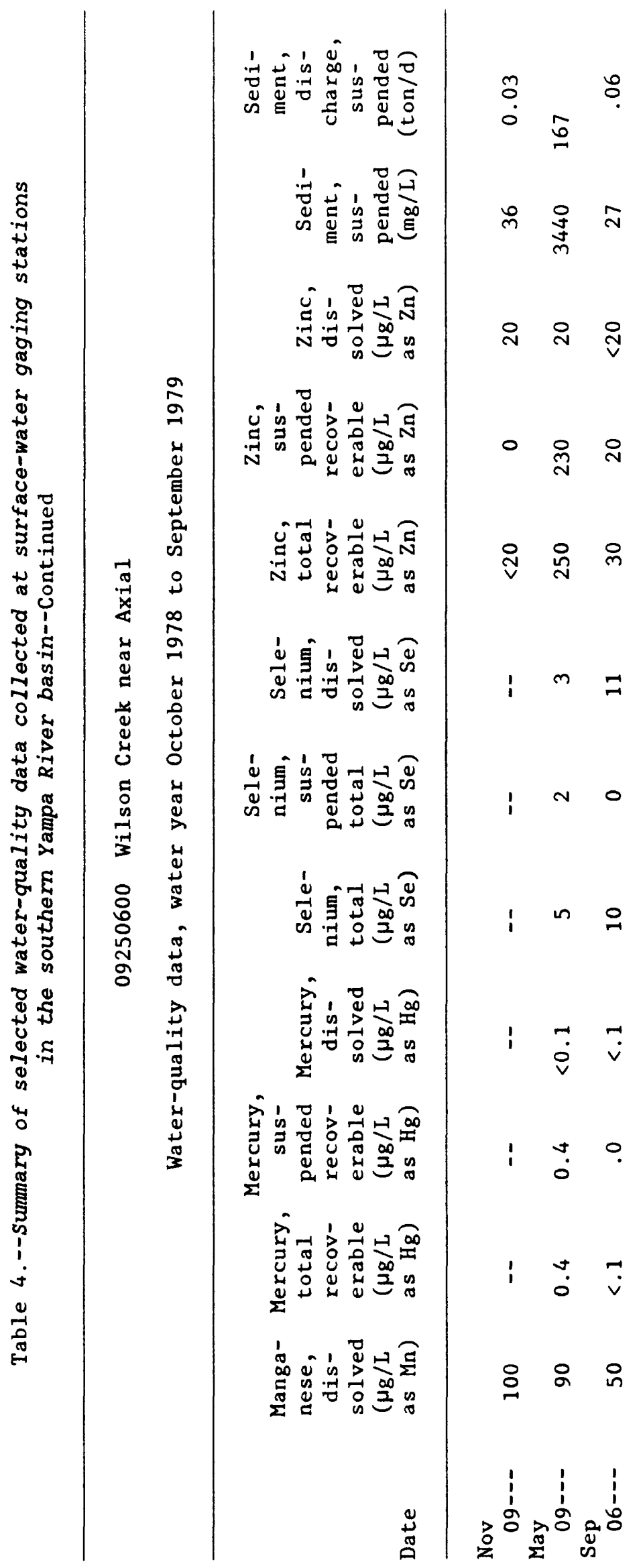




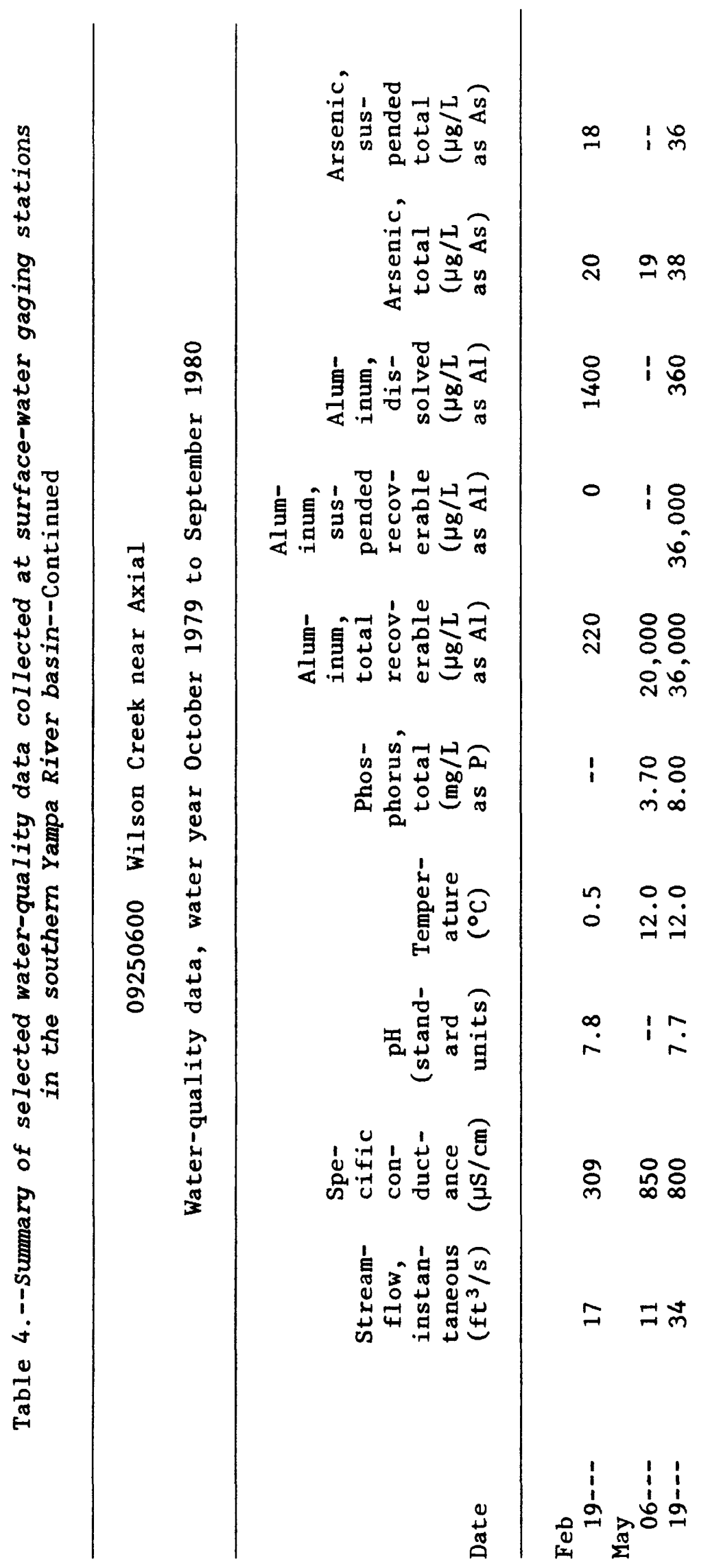




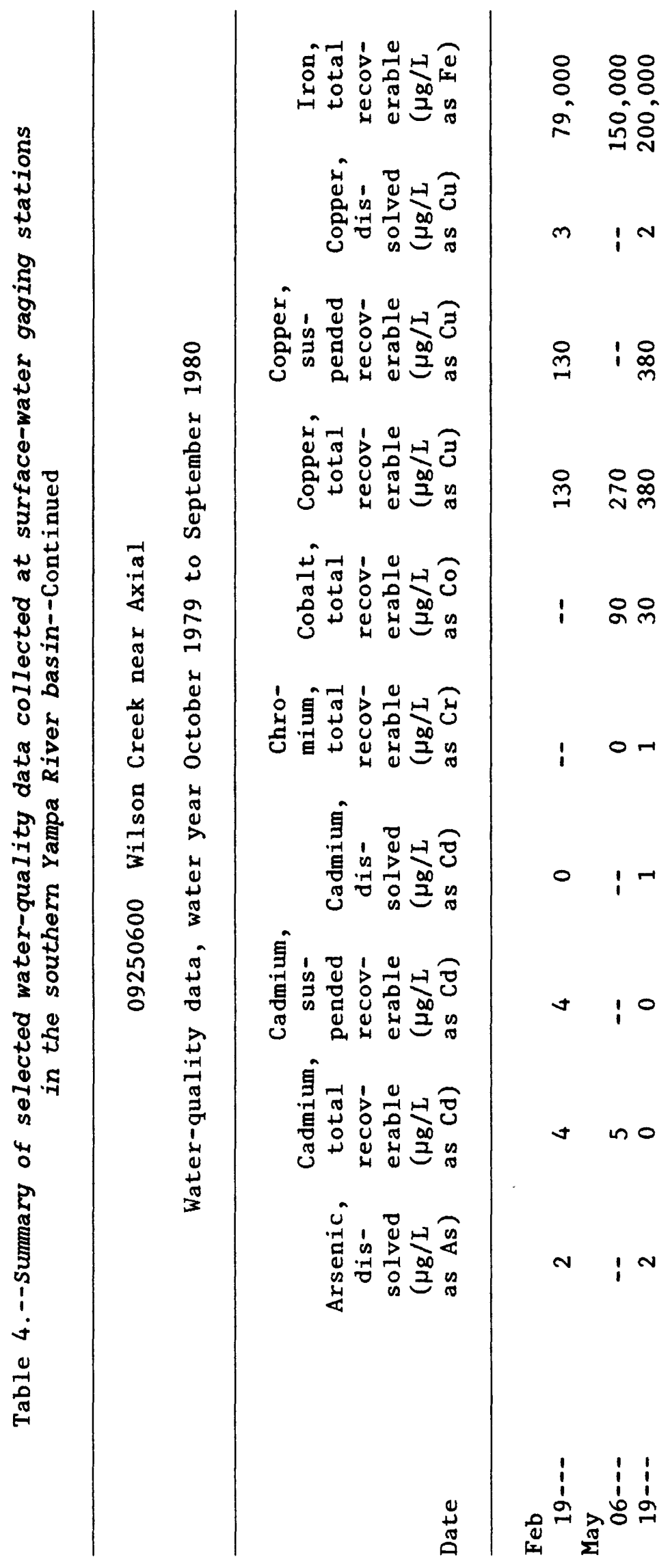




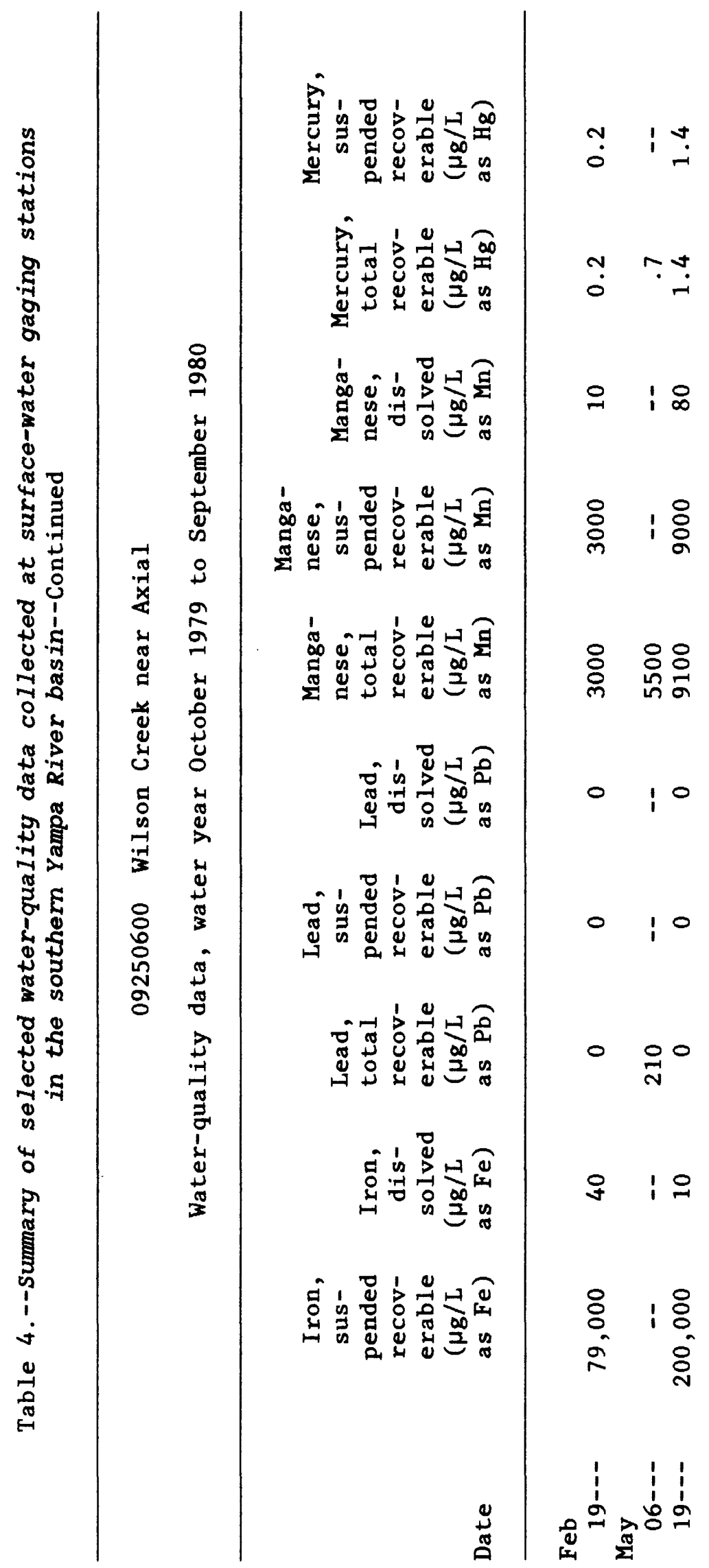




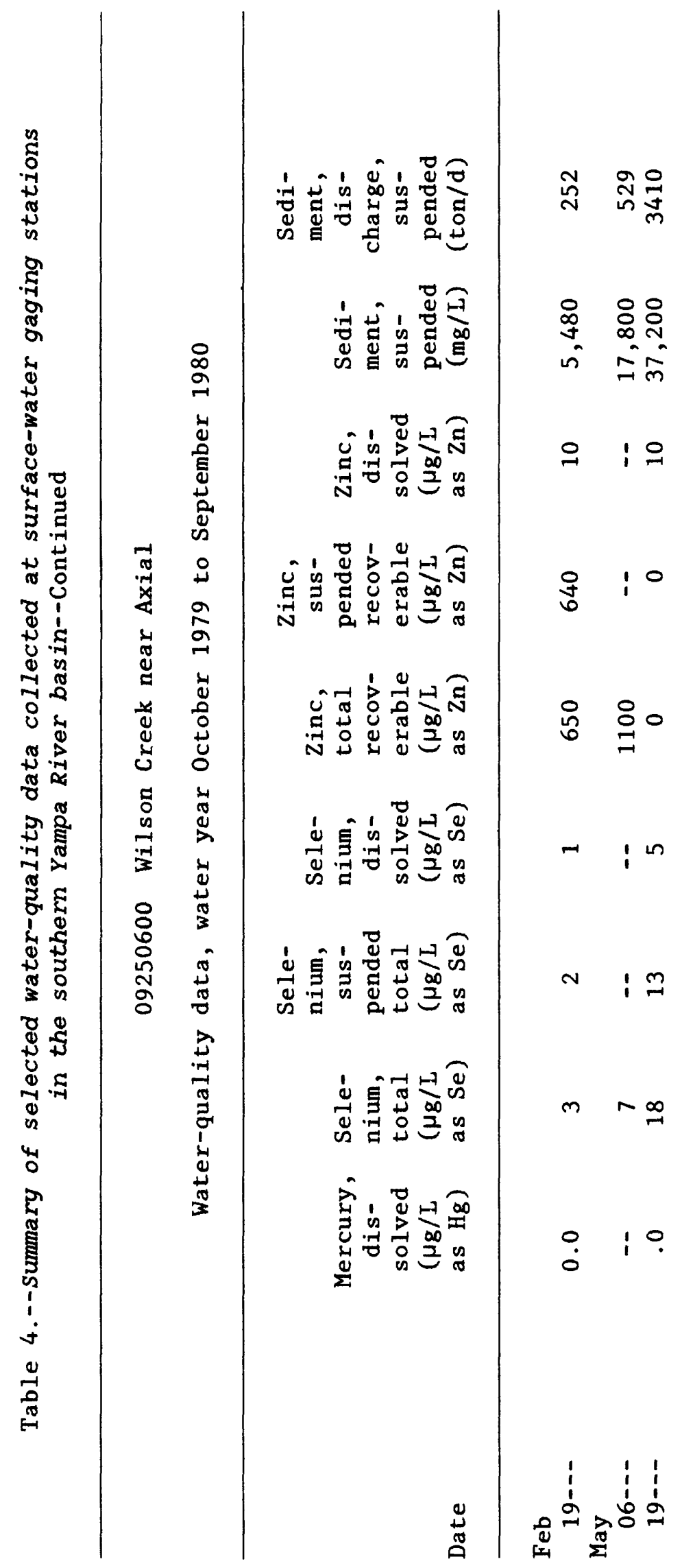




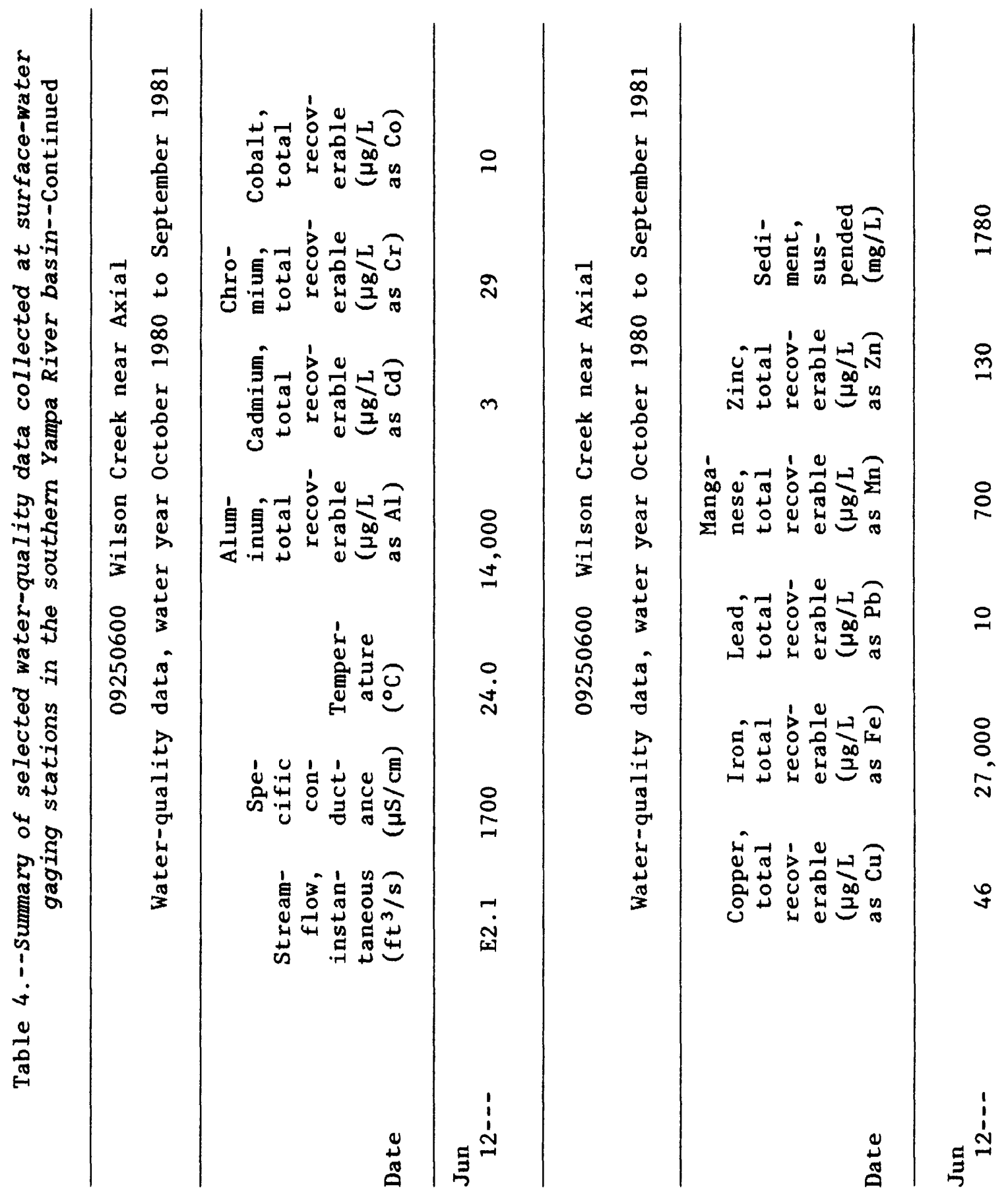




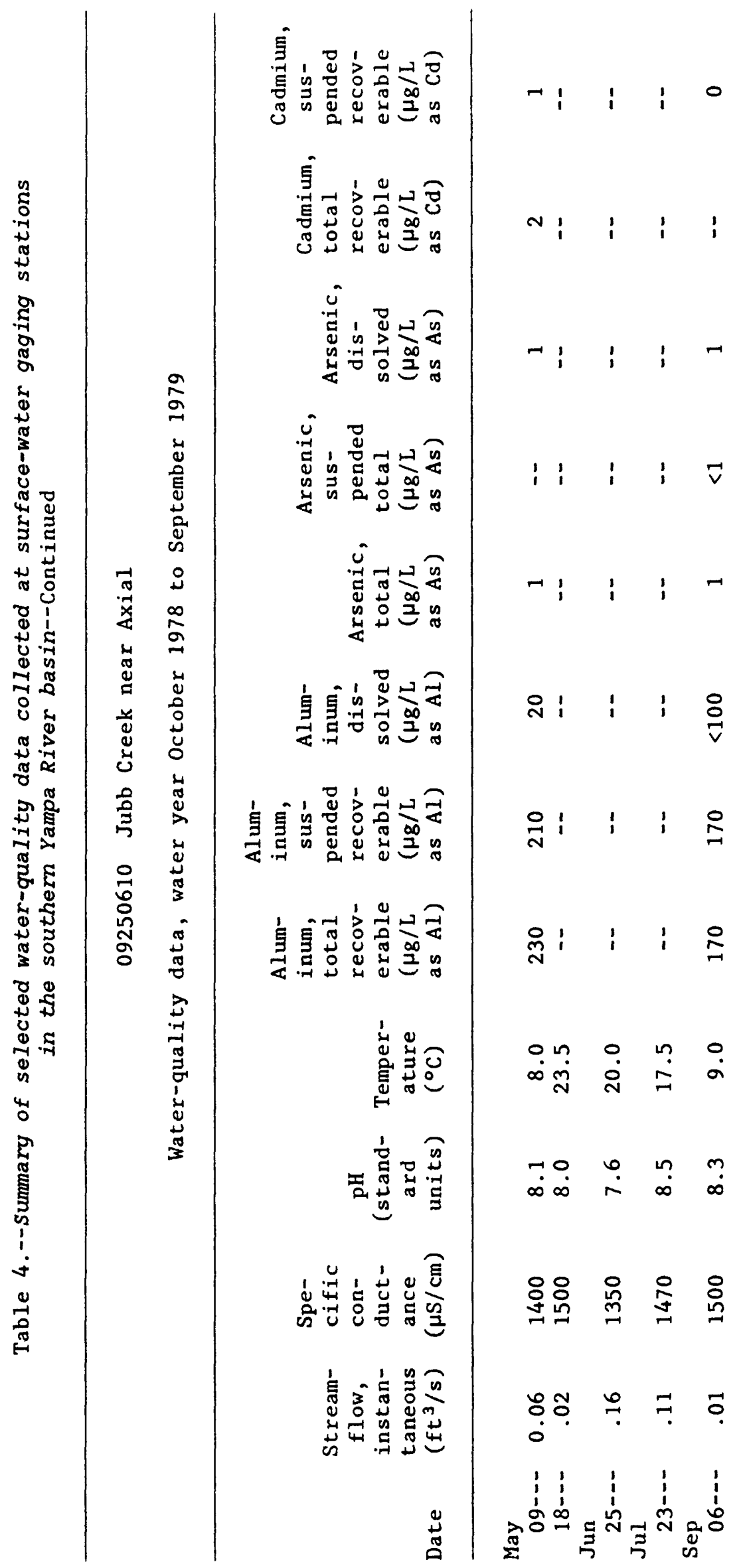




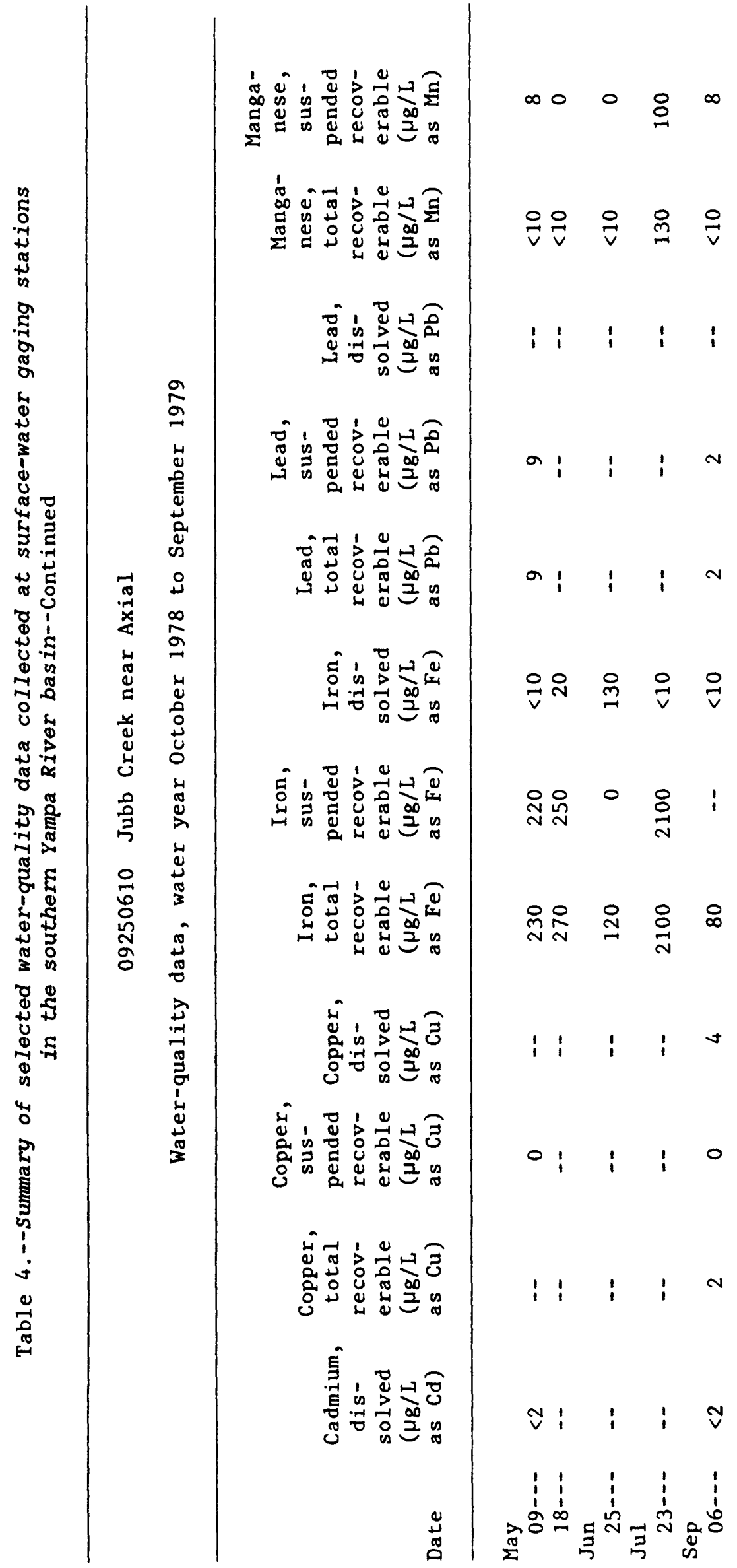




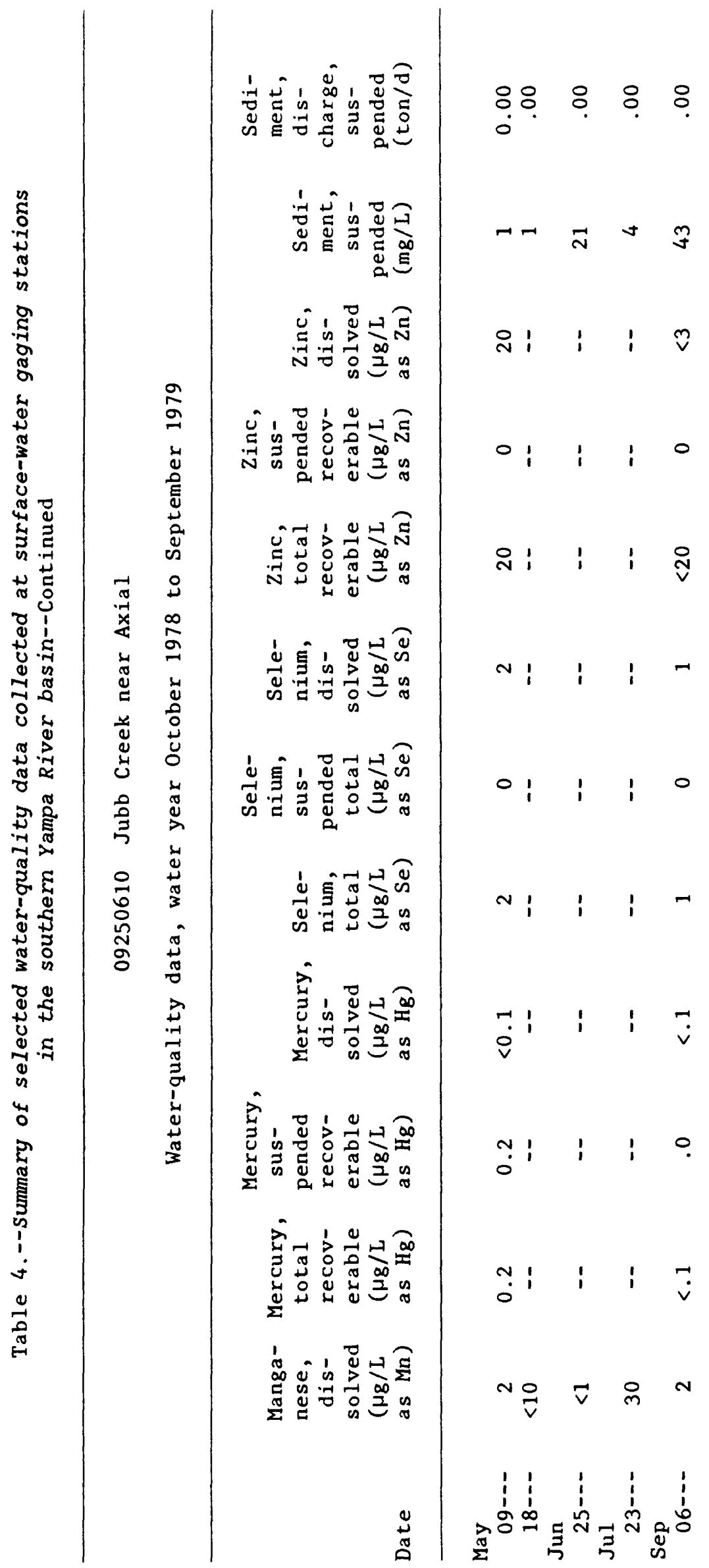




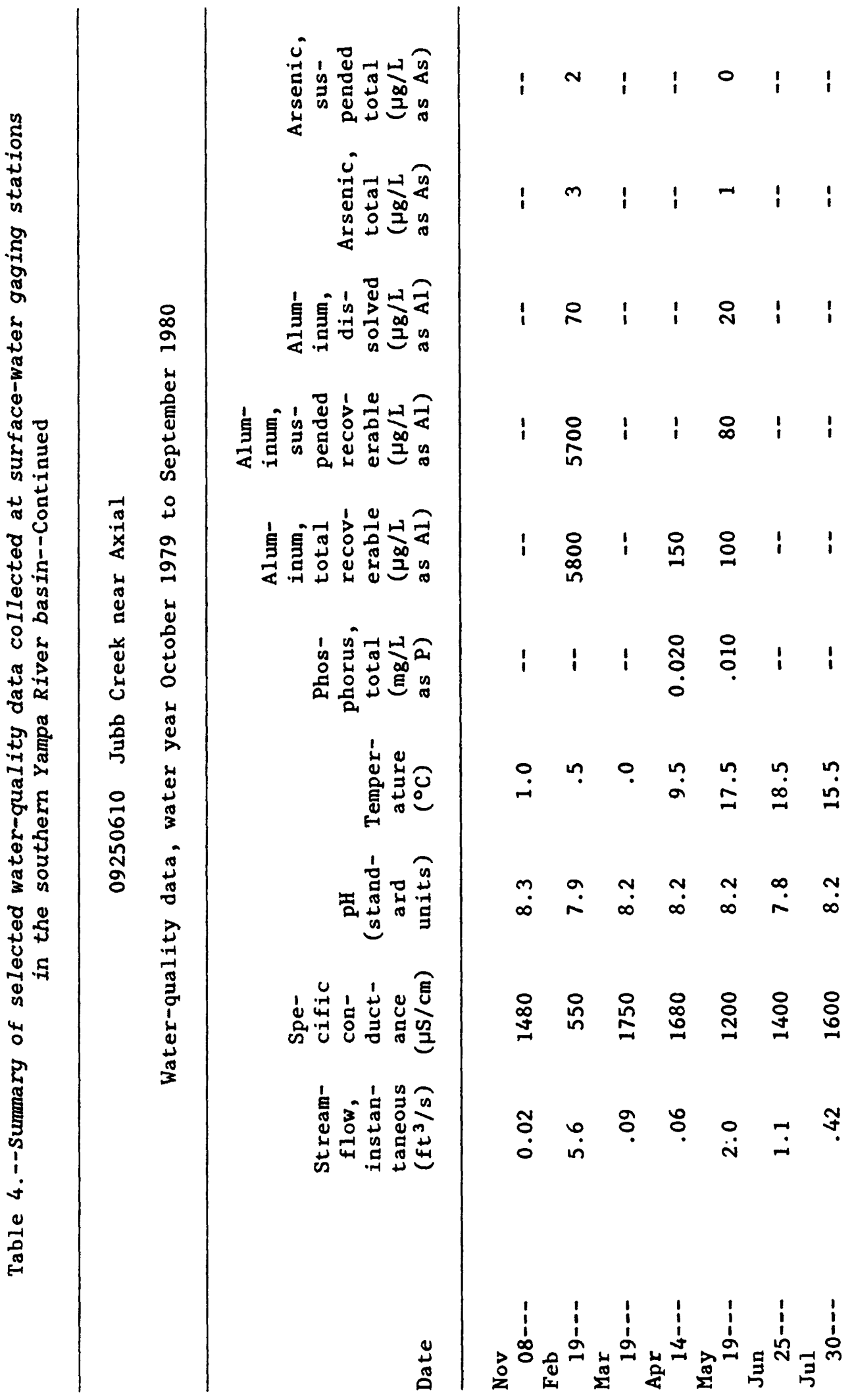




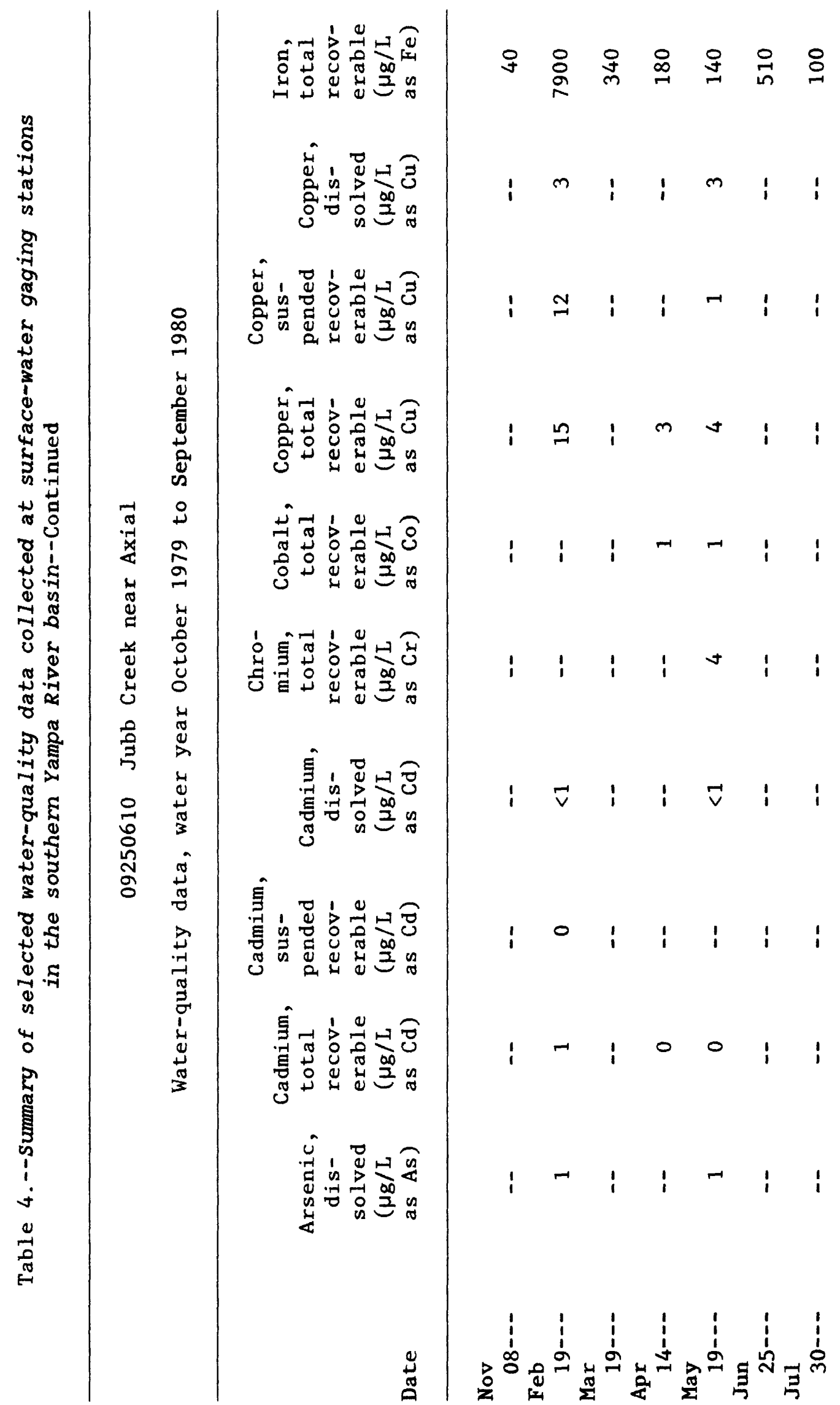




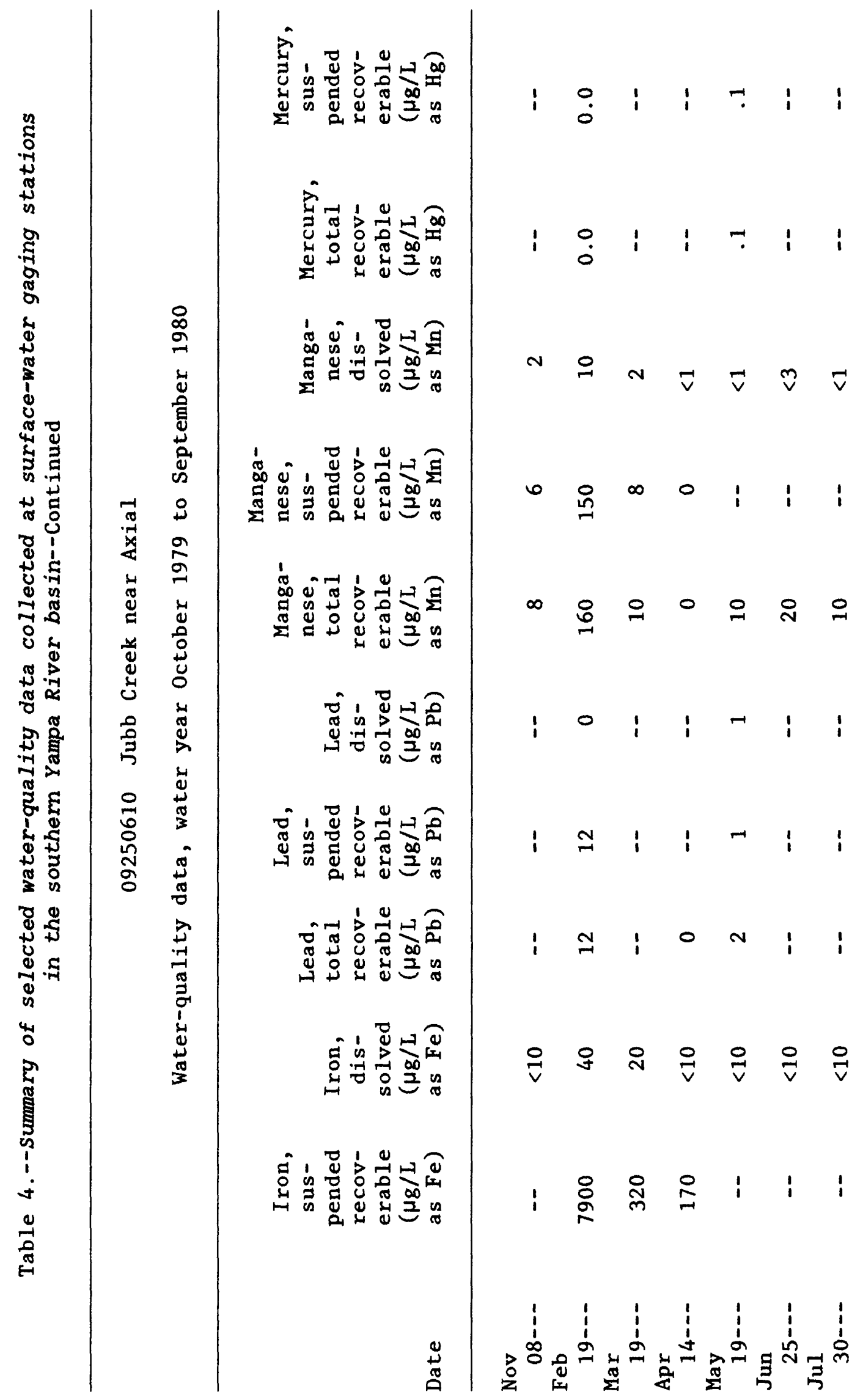




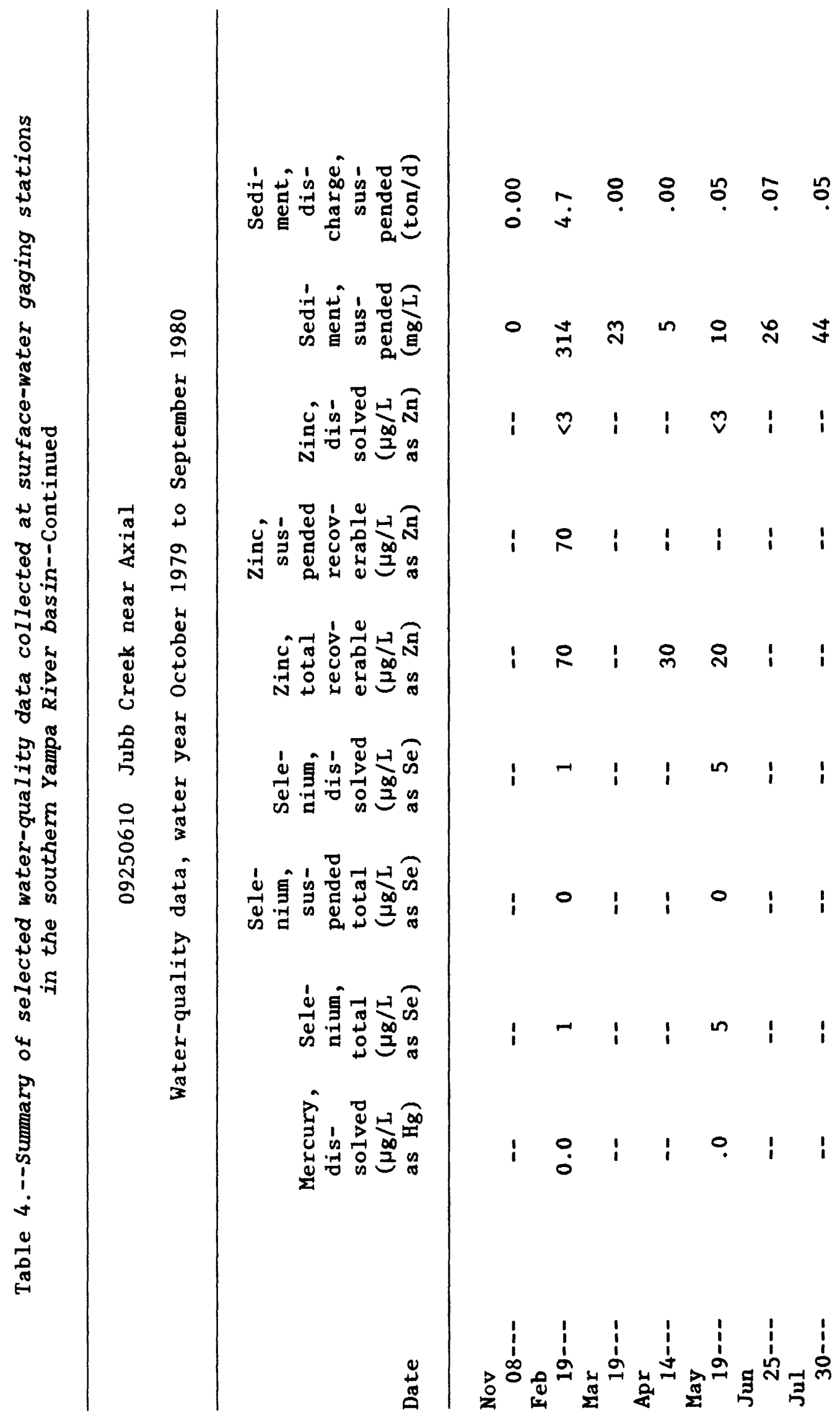




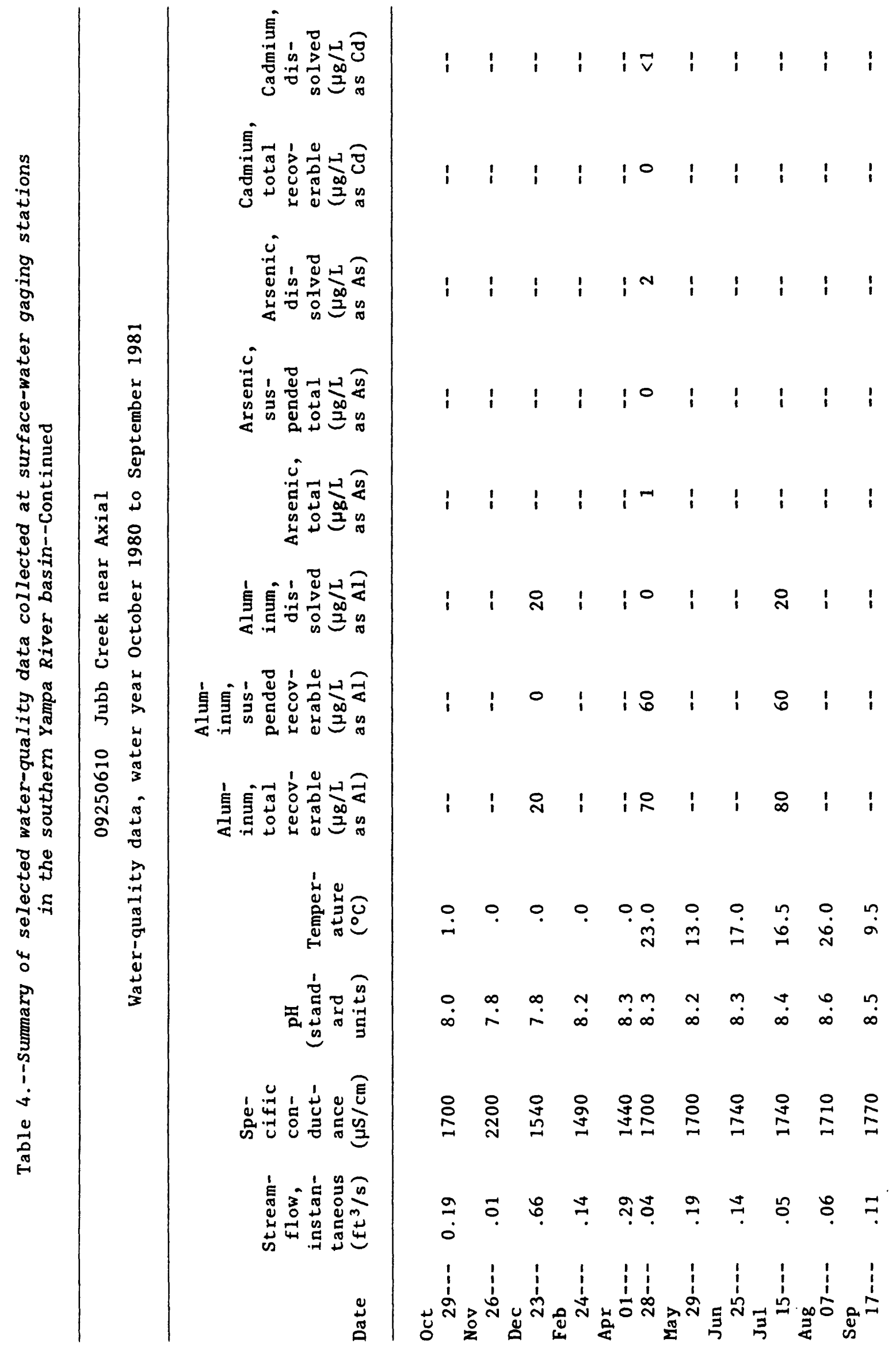




$$
\text { กㅇำ } 00 \text { n }
$$

i.

유 오오 욱으으으으으

2

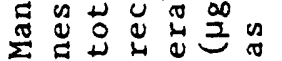

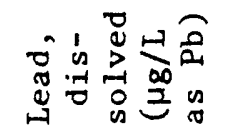

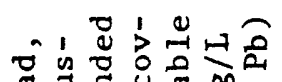

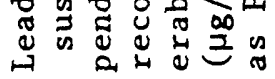

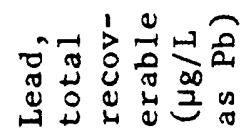

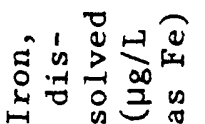

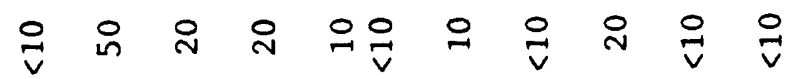

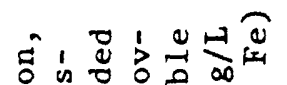

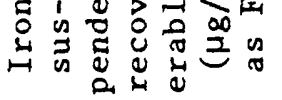

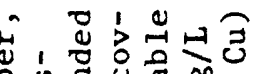

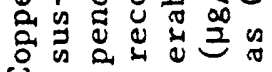

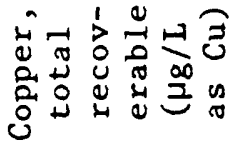




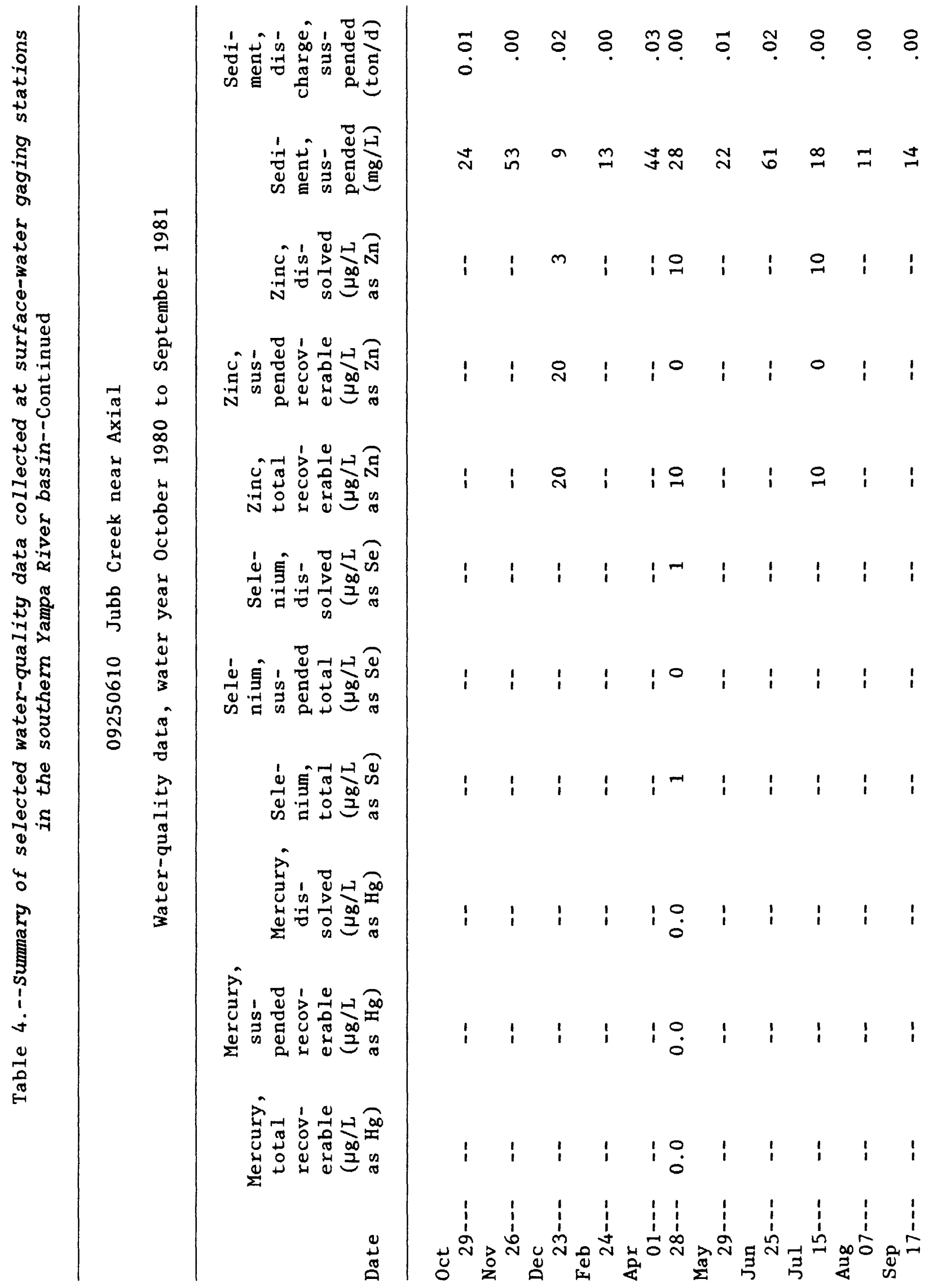




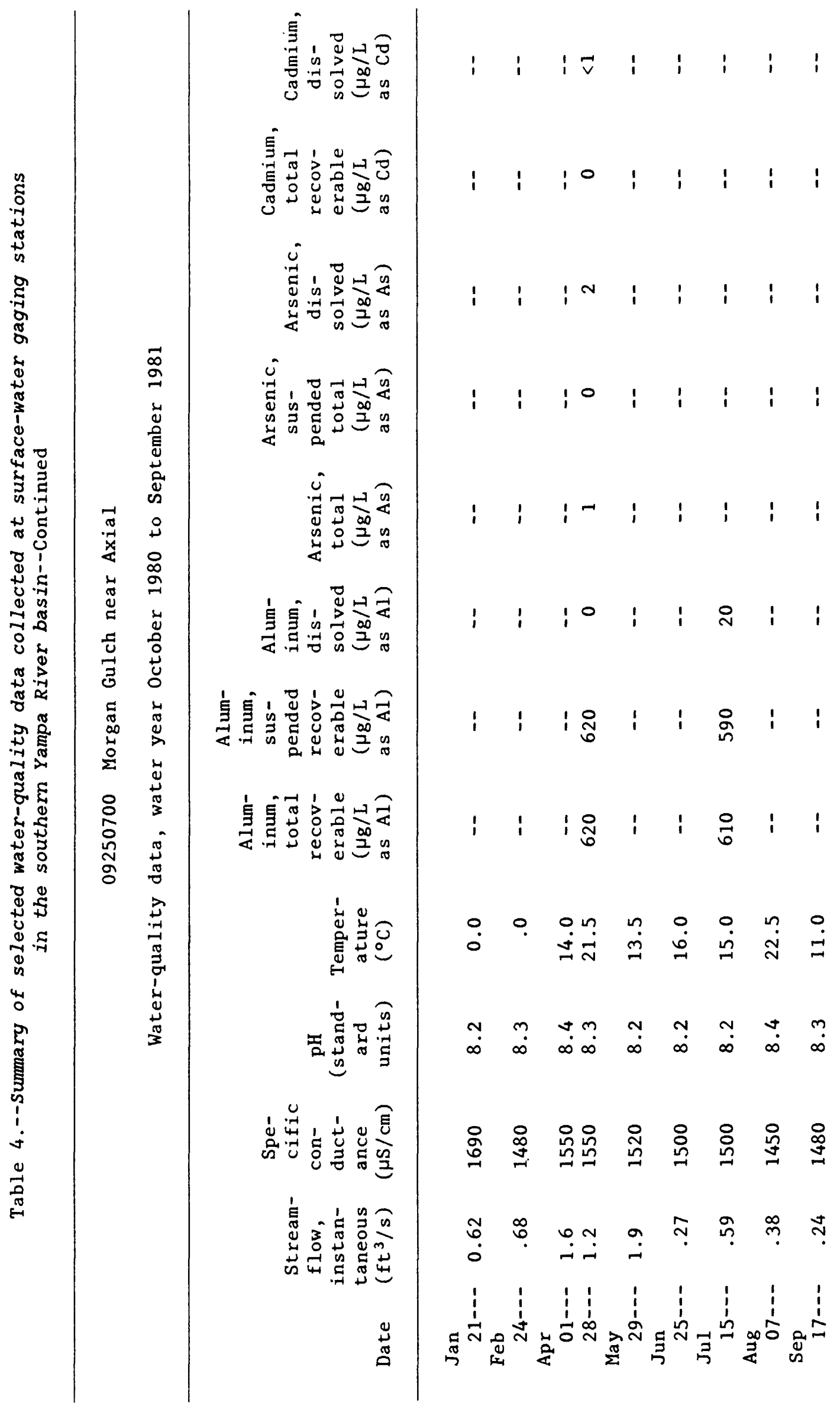




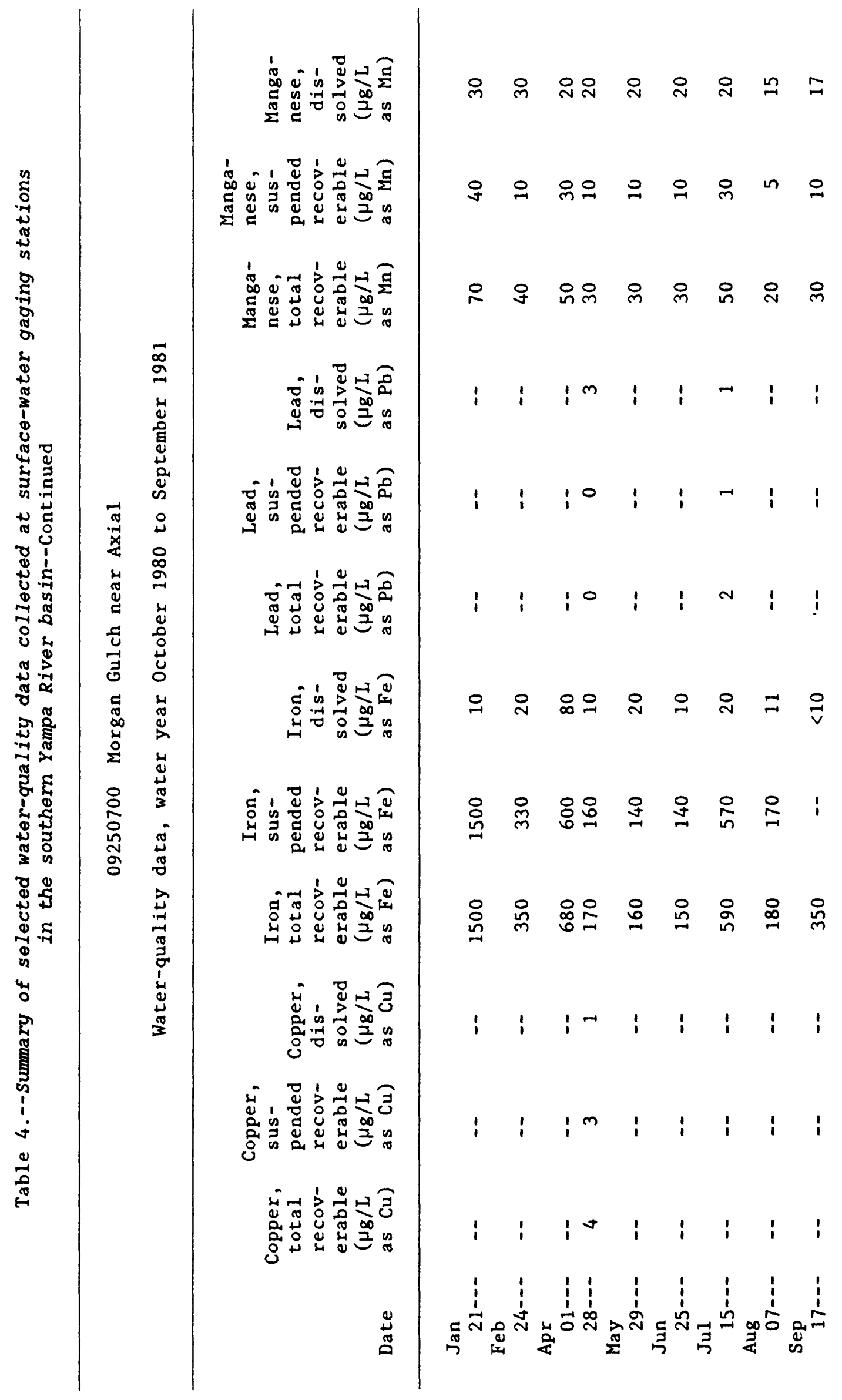




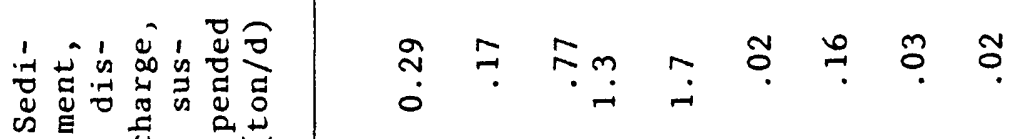

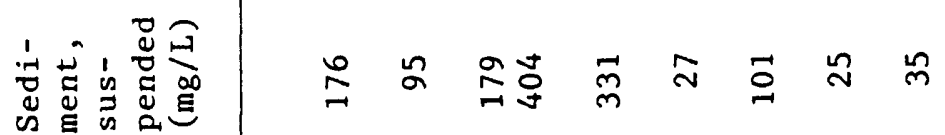

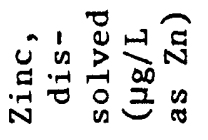

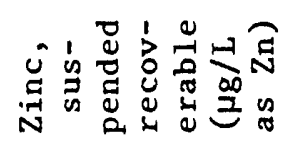

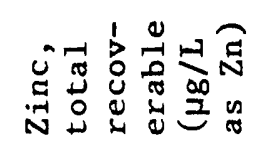

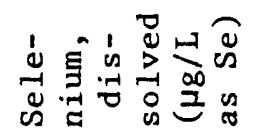

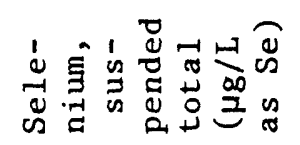

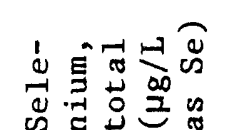

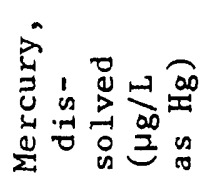

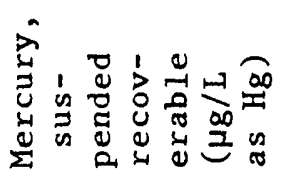

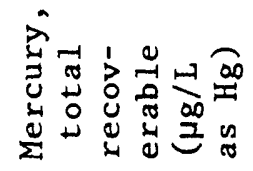

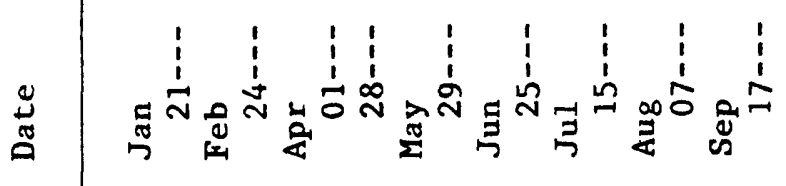




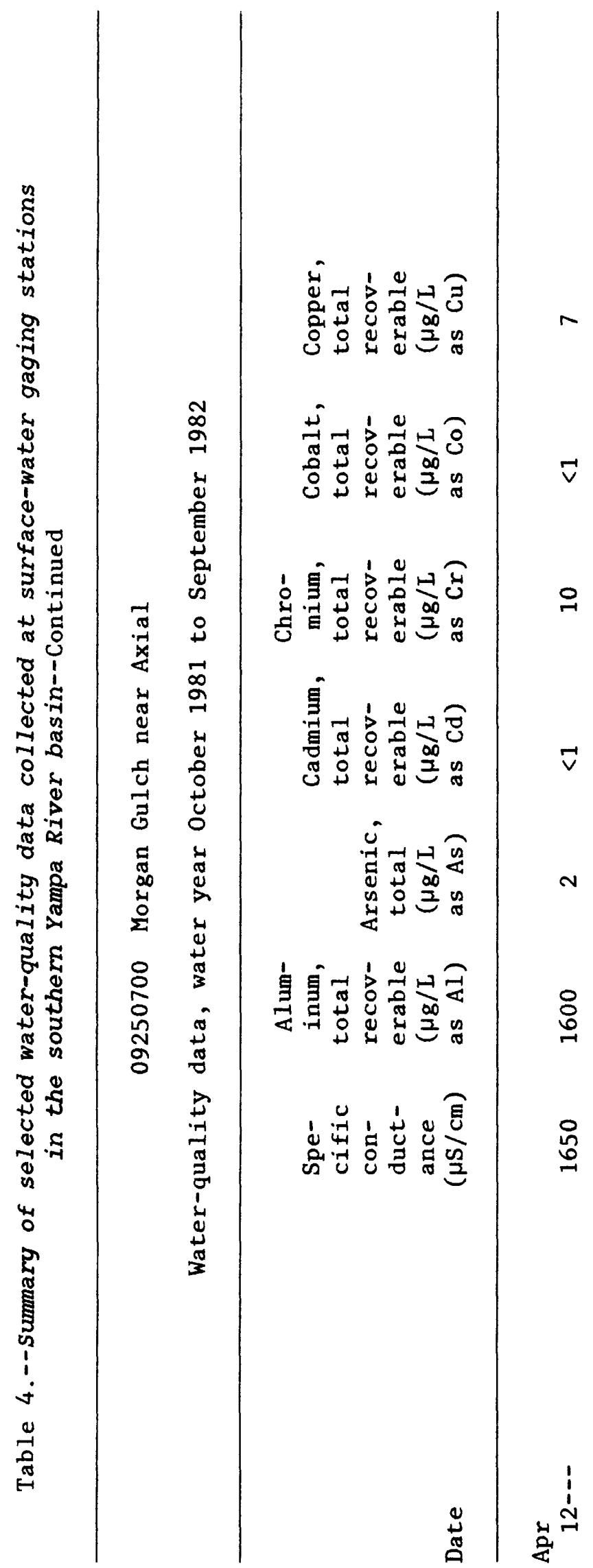

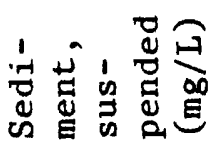

年

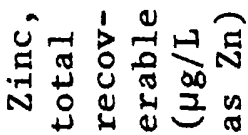

m

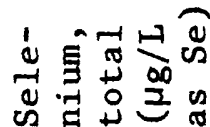

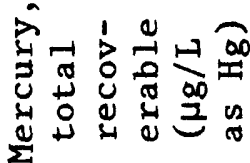

i

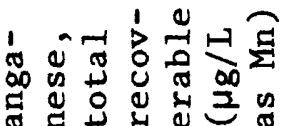

$\infty$

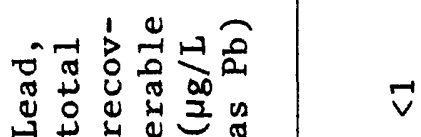

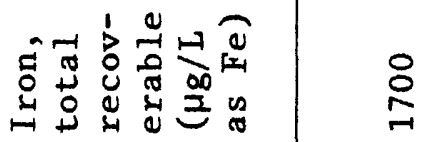


Table 5.--Statistical summary of water-quality data collected at selected surface-water gaging stations in the southern Yampa River basin

09244410 Yampa River below diversion, near Hayden

\begin{tabular}{|c|c|c|c|c|c|c|}
\hline Parameter & $\mathbf{N}$ & Mean & $\begin{array}{l}\text { Standard } \\
\text { deviation }\end{array}$ & $\begin{array}{l}\text { Minimum } \\
\text { value }\end{array}$ & $\begin{array}{l}\text { Maximum } \\
\text { value }\end{array}$ & $\begin{array}{l}\text { Standard } \\
\text { error } \\
\text { of mean }\end{array}$ \\
\hline $\begin{array}{l}\text { Streamflow, instantaneous }\left(\mathrm{ft}^{3} / \mathrm{s}\right) \\
\text { Specific conductance }(\mu \mathrm{S} / \mathrm{cm}) \\
\text { pH (standard units) } \\
\left.\text { Temperature ( }{ }^{\circ} \mathrm{C}\right) \\
\text { Phosphorus, total (mg/L as P) }\end{array}$ & $\begin{array}{r}125 \\
113 \\
88 \\
118 \\
81\end{array}$ & $\begin{array}{r}1250.78 \\
269.40 \\
7.76 \\
7.27 \\
0.07\end{array}$ & $\begin{array}{r}1846.08 \\
115.83 \\
0.44 \\
6.04 \\
0.05\end{array}$ & $\begin{array}{r}38.90 \\
50.00 \\
6.80 \\
0.00 \\
0.01\end{array}$ & $\begin{array}{r}7300.00 \\
600.00 \\
8.60 \\
21.00 \\
0.24\end{array}$ & $\begin{array}{r}165.12 \\
10.90 \\
0.05 \\
0.56 \\
0.01\end{array}$ \\
\hline $\begin{array}{l}\text { Aluminum, total recoverable ( } \mu g / L \text { as } A 1) \\
\text { Aluminum, suspended recoverable }(\mu g / L \text { as } A 1) \\
\text { Aluminum, dissolved ( } \mu g / L \text { as } A 1) \\
\text { Arsenic, total ( } \mu g / L \text { as } A s) \\
\text { Arsenic, suspended total }(\mu g / L \text { as } A s)\end{array}$ & $\begin{array}{l}18 \\
14 \\
15 \\
18 \\
13\end{array}$ & $\begin{array}{r}392.22 \\
243.57 \\
48.00 \\
1.22 \\
0.92\end{array}$ & $\begin{array}{r}423.98 \\
301.75 \\
36.10 \\
0.43 \\
0.28\end{array}$ & $\begin{array}{r}50.00 \\
40.00 \\
10.00 \\
1.00 \\
0.00\end{array}$ & $\begin{array}{r}1300.00 \\
1200.00 \\
100.00 \\
2.00 \\
1.00\end{array}$ & $\begin{array}{r}99.93 \\
80.65 \\
9.32 \\
0.10 \\
0.08\end{array}$ \\
\hline $\begin{array}{l}\text { Arsenic, dissolved ( } \mu \mathrm{g} / \mathrm{L} \text { as } \mathrm{As}) \\
\text { Cadmium, total recoverable }(\mu \mathrm{g} / \mathrm{L} \text { as } \mathrm{Cd}) \\
\text { Cadmium, suspended recoverable }(\mu \mathrm{g} / \mathrm{L} \text { as } \mathrm{C}) \\
\text { Cadmium, dissolved ( } \mu \mathrm{g} / \mathrm{L} \text { as } \mathrm{Cd}) \\
\text { Chromium, total recoverable }(\mu \mathrm{g} / \mathrm{L} \text { as } \mathrm{Cr}) \\
\text { Chromium, suspended recoverable }(\mu \mathrm{g} / \mathrm{L} \text { as } \mathrm{Cr})\end{array}$ & $\begin{array}{l}15 \\
19 \\
13 \\
16 \\
18 \\
14\end{array}$ & $\begin{array}{l}1.40 \\
8.63 \\
6.08 \\
1.56 \\
6.39 \\
0.71\end{array}$ & $\begin{array}{l}0.91 \\
9.97 \\
5.79 \\
1.55 \\
8.71 \\
2.67\end{array}$ & $\begin{array}{l}1.00 \\
0.00 \\
0.00 \\
0.00 \\
0.00 \\
0.00\end{array}$ & $\begin{array}{r}4.00 \\
20.00 \\
18.00 \\
6.00 \\
20.00 \\
10.00\end{array}$ & $\begin{array}{l}0.24 \\
2.29 \\
1.61 \\
0.39 \\
2.05 \\
0.71\end{array}$ \\
\hline $\begin{array}{l}\text { Chromium, dissolved }(\mu g / \mathrm{L} \text { as } \mathrm{Cr}) \\
\text { Cobalt, total recoverable }(\mu g / \mathrm{L} \text { as Co) } \\
\text { Cobalt, suspended recoverable }(\mu \mathrm{g} / \mathrm{L} \text { as } \mathrm{C}) \\
\text { Cobait, dissolved }(\mu \mathrm{g} / \mathrm{L} \text { as } \mathrm{Co}) \\
\text { Copper, total recoverable }(\mu \mathrm{g} / \mathrm{L} \text { as } \mathrm{Cu}) \\
\text { Copper, suspended recoverable }(\mu \mathrm{g} / \mathrm{L} \text { as } \mathrm{C})\end{array}$ & $\begin{array}{r}15 \\
1 \\
1 \\
1 \\
19 \\
16\end{array}$ & $\begin{array}{r}4.60 \\
100.00 \\
50.00 \\
0.00 \\
12.68 \\
6.69\end{array}$ & \begin{tabular}{r}
8.12 \\
\hdashline 9.89 \\
8.34
\end{tabular} & $\begin{array}{r}0.00 \\
100.00 \\
50.00 \\
0.00 \\
0.00 \\
0.00\end{array}$ & $\begin{array}{r}20.00 \\
100.00 \\
50.00 \\
0.00 \\
37.00 \\
35.00\end{array}$ & $\begin{array}{l}2.10 \\
2.27 \\
2.09\end{array}$ \\
\hline $\begin{array}{l}\text { Copper, dissolved }(\mu g / L \text { as } \mathrm{Cu}) \\
\text { Iron, total recoverable }(\mu \mathrm{g} / \mathrm{L} \text { as } \mathrm{Fe}) \\
\text { Iron, suspended recoverable ( } \mu \mathrm{g} / \mathrm{L} \text { as } \mathrm{Fe}) \\
\text { Iron, dissolved ( } \mu \mathrm{g} / \mathrm{L} \text { as } \mathrm{Fe}) \\
\text { Lead, total recoverable }(\mu \mathrm{g} / \mathrm{L} \text { as } \mathrm{Pb})\end{array}$ & $\begin{array}{r}16 \\
10 \\
2 \\
80 \\
19\end{array}$ & $\begin{array}{r}4.13 \\
365.00 \\
80.00 \\
97.42 \\
103.63\end{array}$ & $\begin{array}{r}4.57 \\
209.62 \\
98.99 \\
59.47 \\
107.20\end{array}$ & $\begin{array}{r}0.00 \\
70.00 \\
10.00 \\
20.00 \\
0.00\end{array}$ & $\begin{array}{r}20.00 \\
680.00 \\
150.00 \\
310.00 \\
300.00\end{array}$ & $\begin{array}{r}1.14 \\
66.29 \\
70.00 \\
6.65 \\
24.59\end{array}$ \\
\hline $\begin{array}{l}\text { Lead, suspended recoverable }(\mu \mathrm{g} / \mathrm{L} \text { as } \mathrm{Pb}) \\
\text { Lead, dissolved }(\mu \mathrm{g} / \mathrm{L} \text { as } \mathrm{Pb}) \\
\text { Manganese, total recoverable }(\mu \mathrm{g} / \mathrm{L} \text { as } \mathrm{Mn}) \\
\text { Manganese, suspended recoverable }\left(\mu_{\mathrm{g}} / \mathrm{L} \text { as } \mathrm{Mn}\right) \\
\text { Manganese, dissolved }(\mu \mathrm{g} / \mathrm{L} \text { as } \mathrm{Mn})\end{array}$ & $\begin{array}{l}16 \\
16 \\
19 \\
15 \\
16\end{array}$ & $\begin{array}{r}124.62 \\
4.81 \\
46.32 \\
19.33 \\
22.50\end{array}$ & $\begin{array}{r}244.83 \\
8.20 \\
18.62 \\
16.24 \\
10.00\end{array}$ & $\begin{array}{r}0.00 \\
0.00 \\
10.00 \\
0.00 \\
10.00\end{array}$ & $\begin{array}{r}1000.00 \\
30.00 \\
80.00 \\
50.00 \\
40.00\end{array}$ & $\begin{array}{r}61.21 \\
2.05 \\
4.27 \\
4.19 \\
2.50\end{array}$ \\
\hline $\begin{array}{l}\text { Mercury, total recoverable }(\mu g / L \text { as } \mathrm{Hg}) \\
\text { Mercury, suspended recoverable }(\mu \mathrm{g} / \mathrm{L} \text { as } \mathrm{Hg}) \\
\text { Mercury, dissolved }(\mu \mathrm{g} / \mathrm{L} \text { as } \mathrm{Hg}) \\
\text { Selenium, total }(\mu \mathrm{g} / \mathrm{L} \text { as } \mathrm{Se}) \\
\text { Selenium, suspended total }(\mu \mathrm{g} / \mathrm{L} \text { as } \mathrm{Se})\end{array}$ & $\begin{array}{l}19 \\
15 \\
16 \\
19 \\
15\end{array}$ & $\begin{array}{l}0.43 \\
0.08 \\
0.44 \\
0.89 \\
0.20\end{array}$ & $\begin{array}{l}0.46 \\
0.23 \\
0.34 \\
0.32 \\
0.41\end{array}$ & $\begin{array}{l}0.00 \\
0.00 \\
0.00 \\
0.00 \\
0.00\end{array}$ & $\begin{array}{l}2.10 \\
0.90 \\
1.20 \\
1.00 \\
1.00\end{array}$ & $\begin{array}{l}0.11 \\
0.06 \\
0.09 \\
0.07 \\
0.11\end{array}$ \\
\hline 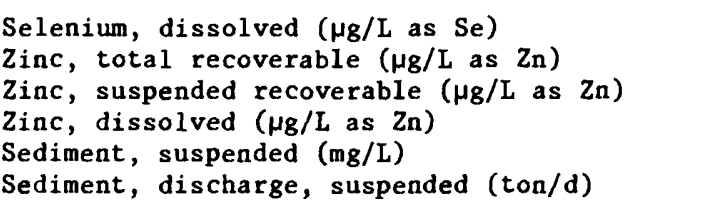 & $\begin{array}{l}16 \\
18 \\
13 \\
15 \\
87 \\
85\end{array}$ & $\begin{array}{r}0.88 \\
35.56 \\
14.62 \\
18.40 \\
48.76 \\
280.60\end{array}$ & $\begin{array}{r}0.34 \\
31.48 \\
20.25 \\
15.67 \\
87.05 \\
607.44\end{array}$ & $\begin{array}{r}0.00 \\
10.00 \\
0.00 \\
0.00 \\
0.00 \\
0.00\end{array}$ & $\begin{array}{r}1.00 \\
110.00 \\
70.00 \\
50.00 \\
648.00 \\
2650.00\end{array}$ & $\begin{array}{r}0.09 \\
7.42 \\
5.62 \\
4.05 \\
9.33 \\
65.89\end{array}$ \\
\hline
\end{tabular}


Table 5.--Statistical summary of water-quality data collected at selected surface-water gaging stations in the southern Yampa River basin--Continued

09246550 Yampa River below Elkhead Creek near Craig

\begin{tabular}{|c|c|c|c|c|c|c|}
\hline Parameter & $\mathrm{N}$ & Mean & $\begin{array}{l}\text { Standard } \\
\text { deviation }\end{array}$ & $\begin{array}{l}\text { Minimum } \\
\text { value }\end{array}$ & $\begin{array}{c}\text { Maximum } \\
\text { value }\end{array}$ & $\begin{array}{l}\text { Standard } \\
\text { error } \\
\text { of mean }\end{array}$ \\
\hline $\begin{array}{l}\left.\text { Streamflow, instantaneous ( } \mathrm{ft}^{3} / \mathrm{s}\right) \\
\text { Specific conductance }(\mu \mathrm{S} / \mathrm{cm}) \\
\text { pH (standard units) } \\
\left.\text { Temperature ( }{ }^{\circ} \mathrm{C}\right) \\
\text { Phosphorus, total (mg/L as } \mathrm{P})\end{array}$ & $\begin{array}{l}38 \\
62 \\
60 \\
62 \\
61\end{array}$ & $\begin{array}{r}1662.24 \\
328.97 \\
7.81 \\
8.30 \\
0.08\end{array}$ & $\begin{array}{r}2671.22 \\
126.49 \\
0.44 \\
7.29 \\
0.10\end{array}$ & $\begin{array}{r}70.00 \\
80.00 \\
6.90 \\
0.00 \\
0.01\end{array}$ & $\begin{array}{r}9900.00 \\
650.00 \\
8.70 \\
22.00 \\
0.59\end{array}$ & $\begin{array}{r}433.33 \\
16.06 \\
0.06 \\
0.93 \\
0.01\end{array}$ \\
\hline $\begin{array}{l}\text { Aluminum, total recoverable }(\mu g / L \text { as } A l) \\
\text { Aluminum, suspended recoverable ( } \mu g / L \text { as } A l) \\
\text { Aluminum, dissolved ( } \mu g / L \text { as } A l) \\
\text { Arsenic, total ( } \mu g / L \text { as As) } \\
\text { Arsenic, suspended total ( } \mu g / L \text { as As) }\end{array}$ & $\begin{array}{l}14 \\
14 \\
15 \\
15 \\
14\end{array}$ & $\begin{array}{r}727.14 \\
719.29 \\
44.67 \\
1.27 \\
0.93\end{array}$ & $\begin{array}{r}1056.87 \\
1060.78 \\
36.03 \\
0.46 \\
0.47\end{array}$ & $\begin{array}{r}70.00 \\
50.00 \\
10.00 \\
1.00 \\
0.00\end{array}$ & $\begin{array}{r}3800.00 \\
3800.00 \\
100.00 \\
2.00 \\
2.00\end{array}$ & $\begin{array}{r}282.46 \\
283.51 \\
9.30 \\
0.12 \\
0.13\end{array}$ \\
\hline $\begin{array}{l}\text { Arsenic, dissolved ( } \mu g / \mathrm{L} \text { as } A s) \\
\text { Cadmium, total recoverable ( } \mu g / L \text { as Cd) } \\
\text { Cadmium, suspended recoverable ( } \mu g / L \text { as C) } \\
\text { Cadmium, dissolved ( } \mu g / L \text { as } C d) \\
\text { Chromium, total recoverable ( } \mu g / L \text { as } C r)\end{array}$ & $\begin{array}{l}15 \\
15 \\
13 \\
16 \\
15\end{array}$ & $\begin{array}{r}1.33 \\
12.20 \\
6.62 \\
1.06 \\
5.33\end{array}$ & $\begin{array}{l}1.05 \\
9.92 \\
4.21 \\
1.06 \\
9.15\end{array}$ & $\begin{array}{l}1.00 \\
0.00 \\
0.00 \\
0.00 \\
0.00\end{array}$ & $\begin{array}{r}5.00 \\
20.00 \\
10.00 \\
3.00 \\
20.00\end{array}$ & $\begin{array}{l}0.27 \\
2.56 \\
1.17 \\
0.27 \\
2.36\end{array}$ \\
\hline $\begin{array}{l}\text { Chromium, suspended recoverable }(\mu g / L \text { as } C r) \\
\text { Chromium, dissolved ( } \mu g / L \text { as } C r) \\
\text { Cobalt, total recoverable }(\mu g / L \text { as Co }) \\
\text { Cobalt, suspended recoverable }(\mu g / L \text { as } C) \\
\text { Cobalt, dissolved }(\mu g / L \text { as Co })\end{array}$ & $\begin{array}{r}15 \\
15 \\
1 \\
1 \\
1\end{array}$ & $\begin{array}{r}1.73 \\
2.93 \\
100.00 \\
50.00 \\
0.00\end{array}$ & $\begin{array}{r}3.69 \\
7.00 \\
-\ldots-0 \\
-. .-0\end{array}$ & $\begin{array}{r}0.00 \\
0.00 \\
100.00 \\
50.00 \\
0.00\end{array}$ & $\begin{array}{r}10.00 \\
20.00 \\
100.00 \\
50.00 \\
0.00\end{array}$ & $\begin{array}{r}0.95 \\
1.81 \\
-. .-\end{array}$ \\
\hline $\begin{array}{l}\text { Copper, total recoverable }(\mu g / L \text { as } \mathrm{Cu}) \\
\text { Copper, suspended recoverable }(\mu g / \mathrm{L} \text { as } \mathrm{C}) \\
\text { Copper, dissolved ( } \mu \mathrm{g} / \mathrm{L} \text { as } \mathrm{Cu}) \\
\text { Iron, total recoverable }(\mu g / \mathrm{L} \text { as } \mathrm{Fe}) \\
\text { Iron, suspended recoverable }(\mu g / \mathrm{L} \text { as } \mathrm{Fe})\end{array}$ & $\begin{array}{r}16 \\
16 \\
16 \\
10 \\
2\end{array}$ & $\begin{array}{r}15.31 \\
6.69 \\
3.88 \\
613.00 \\
105.00\end{array}$ & $\begin{array}{r}6.76 \\
4.78 \\
4.59 \\
665.80 \\
91.92\end{array}$ & $\begin{array}{r}2.00 \\
0.00 \\
0.00 \\
70.00 \\
40.00\end{array}$ & $\begin{array}{r}20.00 \\
19.00 \\
20.00 \\
2300.00 \\
170.00\end{array}$ & $\begin{array}{r}1.69 \\
1.20 \\
1.15 \\
210.54 \\
65.00\end{array}$ \\
\hline 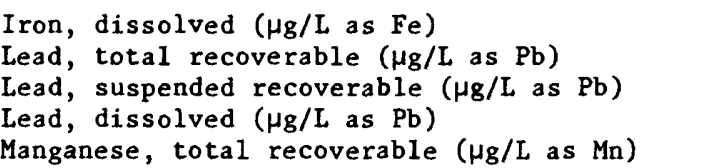 & $\begin{array}{l}60 \\
16 \\
16 \\
16 \\
16\end{array}$ & $\begin{array}{r}95.50 \\
103.63 \\
51.50 \\
2.38 \\
71.88\end{array}$ & $\begin{array}{r}80.45 \\
99.70 \\
48.58 \\
5.37 \\
80.43\end{array}$ & $\begin{array}{r}10.00 \\
0.00 \\
0.00 \\
0.00 \\
20.00\end{array}$ & $\begin{array}{r}510.00 \\
200.00 \\
100.00 \\
22.00 \\
360.00\end{array}$ & $\begin{array}{r}10.39 \\
24.93 \\
12.14 \\
1.34 \\
20.11\end{array}$ \\
\hline $\begin{array}{l}\text { Manganese, suspended recoverable }(\mu g / L \text { as } \mathrm{Mn}) \\
\text { Manganese, dissolved }(\mu g / L \text { as } \mathrm{Mn}) \\
\text { Mercury, total recoverable }(\mu \mathrm{g} / \mathrm{L} \text { as } \mathrm{Hg}) \\
\text { Mercury, suspended recoverable }(\mu \mathrm{g} / \mathrm{L} \text { as } \mathrm{Hg}) \\
\text { Mercury, dissolved ( } \mu \mathrm{g} / \mathrm{L} \text { as } \mathrm{Hg})\end{array}$ & $\begin{array}{l}16 \\
16 \\
16 \\
16 \\
16\end{array}$ & $\begin{array}{r}52.50 \\
20.63 \\
0.77 \\
0.29 \\
0.49\end{array}$ & $\begin{array}{r}74.16 \\
12.89 \\
1.20 \\
0.75 \\
0.51\end{array}$ & $\begin{array}{r}10.00 \\
10.00 \\
0.00 \\
0.00 \\
0.00\end{array}$ & $\begin{array}{r}320.00 \\
50.00 \\
4.50 \\
2.30 \\
2.20\end{array}$ & $\begin{array}{r}18.54 \\
3.22 \\
0.30 \\
0.19 \\
0.13\end{array}$ \\
\hline $\begin{array}{l}\text { Selenium, total ( } \mu g / L \text { as } \mathrm{Se}) \\
\text { Selenium, suspended total ( } \mu \mathrm{g} / \mathrm{L} \text { as } \mathrm{Se}) \\
\text { Selenium, dissolved ( } \mu \mathrm{g} / \mathrm{L} \text { as } \mathrm{Se}) \\
\mathrm{Zinc} \text {, total recoverable }(\mu \mathrm{g} / \mathrm{L} \text { as } \mathrm{Zn}) \\
\mathrm{Zinc} \text {, suspended recoverable }(\mu \mathrm{g} / \mathrm{L} \text { as } \mathrm{Zn})\end{array}$ & $\begin{array}{l}16 \\
15 \\
16 \\
16 \\
15\end{array}$ & $\begin{array}{r}1.00 \\
0.13 \\
1.00 \\
30.00 \\
16.00\end{array}$ & $\begin{array}{r}0.37 \\
0.35 \\
0.37 \\
19.32 \\
15.02\end{array}$ & $\begin{array}{l}0.00 \\
0.00 \\
0.00 \\
0.00 \\
0.00\end{array}$ & $\begin{array}{r}2.00 \\
1.00 \\
2.00 \\
80.00 \\
50.00\end{array}$ & $\begin{array}{l}0.09 \\
0.09 \\
0.09 \\
4.83 \\
3.88\end{array}$ \\
\hline $\begin{array}{l}\text { Zinc, dissolved }(\mu g / L \text { as } Z n) \\
\text { Sediment, suspended }(\mathrm{mg} / \mathrm{L}) \\
\text { Sediment, discharge, suspended (ton/d) }\end{array}$ & $\begin{array}{r}16 \\
6 \\
4\end{array}$ & $\begin{array}{l}14.25 \\
16.50 \\
16.60\end{array}$ & $\begin{array}{l}11.50 \\
13.37 \\
17.35\end{array}$ & $\begin{array}{l}0.00 \\
5.00 \\
3.80\end{array}$ & $\begin{array}{l}40.00 \\
38.00 \\
41.00\end{array}$ & $\begin{array}{l}2.88 \\
5.46 \\
8.68\end{array}$ \\
\hline
\end{tabular}


Table 5.--Statistical summary of water-quality data collected at selected surface-water gaging stations in the southern Yampa River basin--Continued

09247600 Yampa River below Craig

\begin{tabular}{|c|c|c|c|c|c|c|}
\hline Parameter & $\mathbf{N}$ & Mean & $\begin{array}{l}\text { Standard } \\
\text { deviation }\end{array}$ & $\begin{array}{l}\text { Minimum } \\
\text { value }\end{array}$ & $\begin{array}{l}\text { Maximum } \\
\text { value }\end{array}$ & $\begin{array}{l}\text { Standard } \\
\text { error } \\
\text { of mean }\end{array}$ \\
\hline $\begin{array}{l}\text { Streamflow, instantaneous }\left(\mathrm{ft}^{3} / \mathrm{s}\right) \\
\text { Specific conductance }(\mu \mathrm{S} / \mathrm{cm}) \\
\text { pH (standard units) } \\
\text { Temperature }\left({ }^{\circ} \mathrm{C}\right) \\
\text { Phosphorus, total (mg/L as P) }\end{array}$ & $\begin{array}{l}72 \\
60 \\
60 \\
60 \\
60\end{array}$ & $\begin{array}{r}1690.14 \\
333.40 \\
8.00 \\
9.26 \\
0.11\end{array}$ & $\begin{array}{r}2627.01 \\
139.08 \\
0.55 \\
7.75 \\
0.07\end{array}$ & $\begin{array}{r}10.70 \\
78.00 \\
7.00 \\
0.00 \\
0.01\end{array}$ & $\begin{array}{r}9990.00 \\
670.00 \\
9.00 \\
24.00 \\
0.27\end{array}$ & $\begin{array}{r}309.60 \\
17.96 \\
0.07 \\
1.00 \\
0.01\end{array}$ \\
\hline $\begin{array}{l}\text { Aluminum, total recoverable }(\mu \mathrm{g} / \mathrm{L} \text { as } \mathrm{Al}) \\
\text { Aluminum, suspended recoverable ( } \mu \mathrm{g} / \mathrm{L} \text { as } \mathrm{Al}) \\
\text { Aluminum, dissolved ( } \mu \mathrm{g} / \mathrm{L} \text { as } \mathrm{Al}) \\
\text { Arsenic, total ( } \mu \mathrm{g} / \mathrm{L} \text { as As) } \\
\text { Arsenic, suspended total ( } \mu \mathrm{g} / \mathrm{L} \text { as As) }\end{array}$ & $\begin{array}{l}12 \\
10 \\
13 \\
13 \\
10\end{array}$ & $\begin{array}{r}963.33 \\
1063.00 \\
40.00 \\
1.54 \\
1.00\end{array}$ & $\begin{array}{r}1422.46 \\
1500.41 \\
24.49 \\
0.66 \\
0.47\end{array}$ & $\begin{array}{r}80.00 \\
30.00 \\
10.00 \\
1.00 \\
0.00\end{array}$ & $\begin{array}{r}4800.00 \\
4700.00 \\
80.00 \\
3.00 \\
2.00\end{array}$ & $\begin{array}{r}410.63 \\
474.47 \\
6.79 \\
0.18 \\
0.15\end{array}$ \\
\hline $\begin{array}{l}\text { Arsenic, dissolved ( } \mu \mathrm{g} / \mathrm{L} \text { as } \mathrm{As}) \\
\text { Cadmium, total recoverable }(\mu \mathrm{g} / \mathrm{L} \text { as } \mathrm{Cd}) \\
\text { Cadmium, suspended recoverable }(\mu \mathrm{g} / \mathrm{L} \text { as } \mathrm{C}) \\
\text { Cadmium, dissolved }(\mu \mathrm{g} / \mathrm{L} \text { as } \mathrm{Cd}) \\
\text { Chromium, total recoverable }(\mu \mathrm{g} / \mathrm{L} \text { as } \mathrm{Cr})\end{array}$ & $\begin{array}{l}13 \\
14 \\
11 \\
14 \\
13\end{array}$ & $\begin{array}{r}1.08 \\
8.79 \\
4.36 \\
1.00 \\
11.15\end{array}$ & $\begin{array}{r}0.28 \\
10.11 \\
4.67 \\
0.96 \\
9.61\end{array}$ & $\begin{array}{l}1.00 \\
0.00 \\
0.00 \\
0.00 \\
0.00\end{array}$ & $\begin{array}{r}2.00 \\
20.00 \\
10.00 \\
2.00 \\
20.00\end{array}$ & $\begin{array}{l}0.08 \\
2.70 \\
1.41 \\
0.26 \\
2.66\end{array}$ \\
\hline $\begin{array}{l}\text { Chromium, suspended recoverable }(\mu \mathrm{g} / \mathrm{L} \text { as } \mathrm{Cr}) \\
\text { Chromium, dissolved }(\mu \mathrm{g} / \mathrm{L} \text { as } \mathrm{Cr}) \\
\text { Cobalt, total recoverable }(\mu \mathrm{g} / \mathrm{L} \text { as Co) } \\
\text { Cobalt, suspended recoverable }(\mu \mathrm{g} / \mathrm{L} \text { as } \mathrm{C}) \\
\text { Cobalt, dissolved }(\mu \mathrm{g} / \mathrm{L} \text { as Co })\end{array}$ & $\begin{array}{r}12 \\
13 \\
1 \\
1 \\
1\end{array}$ & $\begin{array}{r}4.17 \\
4.62 \\
100.00 \\
50.00 \\
0.00\end{array}$ & $\begin{array}{l}6.69 \\
8.77 \\
-. .- \\
-.-1\end{array}$ & $\begin{array}{r}0.00 \\
0.00 \\
100.00 \\
50.00 \\
0.00\end{array}$ & $\begin{array}{r}20.00 \\
20.00 \\
100.00 \\
50.00 \\
0.00\end{array}$ & $\begin{array}{l}1.93 \\
2.43 \\
-\cdots- \\
-.-2 \\
--2\end{array}$ \\
\hline $\begin{array}{l}\text { Copper, total recoverable }(\mu g / L \text { as } \mathrm{Cu}) \\
\text { Copper, suspended recoverable }(\mu \mathrm{g} / \mathrm{L} \text { as } \mathrm{C}) \\
\text { Copper, dissolved }(\mu \mathrm{g} / \mathrm{L} \text { as } \mathrm{Cu}) \\
\text { Iron, total recoverable }(\mu \mathrm{L} / \mathrm{L} \text { as } \mathrm{Fe}) \\
\text { Iron, suspended recoverable }(\mu \mathrm{g} / \mathrm{L} \text { as } \mathrm{Fe})\end{array}$ & $\begin{array}{r}14 \\
13 \\
14 \\
8 \\
2\end{array}$ & $\begin{array}{r}13.50 \\
7.77 \\
3.21 \\
1135.00 \\
75.00\end{array}$ & $\begin{array}{r}9.19 \\
6.94 \\
3.24 \\
1692.18 \\
49.50\end{array}$ & $\begin{array}{r}0.00 \\
0.00 \\
0.00 \\
70.00 \\
40.00\end{array}$ & $\begin{array}{r}31.00 \\
27.00 \\
13.00 \\
5000.00 \\
110.00\end{array}$ & $\begin{array}{r}2.46 \\
1.93 \\
0.87 \\
598.27 \\
35.00\end{array}$ \\
\hline $\begin{array}{l}\text { Iron, dissolved }(\mu \mathrm{g} / \mathrm{L} \text { as } \mathrm{Fe}) \\
\text { Lead, total recoverable }(\mu \mathrm{g} / \mathrm{L} \text { as } \mathrm{Pb}) \\
\text { Lead, suspended recoverable ( } \mu \mathrm{g} / \mathrm{L} \text { as } \mathrm{Pb}) \\
\text { Lead, dissolved }(\mu \mathrm{L} / \mathrm{L} \text { as } \mathrm{Pb}) \\
\text { Manganese, total recoverable ( } \mu \mathrm{g} / \mathrm{L} \text { as } \mathrm{Mn})\end{array}$ & $\begin{array}{l}58 \\
14 \\
13 \\
14 \\
14\end{array}$ & $\begin{array}{r}91.38 \\
89.36 \\
40.62 \\
2.00 \\
71.43\end{array}$ & $\begin{array}{r}71.04 \\
99.56 \\
47.15 \\
3.11 \\
41.30\end{array}$ & $\begin{array}{r}10.00 \\
0.00 \\
0.00 \\
0.00 \\
20.00\end{array}$ & $\begin{array}{r}370.00 \\
200.00 \\
99.00 \\
12.00 \\
170.00\end{array}$ & $\begin{array}{r}9.33 \\
26.61 \\
13.08 \\
0.83 \\
11.04\end{array}$ \\
\hline $\begin{array}{l}\text { Manganese, suspended recoverable }(\mu \mathrm{g} / \mathrm{L} \text { as } \mathrm{Mn}) \\
\text { Manganese, dissolved }(\mu \mathrm{g} / \mathrm{L} \text { as } \mathrm{Mn}) \\
\text { Mercury, total recoverable }(\mu \mathrm{g} / \mathrm{L} \text { as } \mathrm{Hg}) \\
\text { Mercury, suspended recoverable }(\mu \mathrm{g} / \mathrm{L} \text { as } \mathrm{Hg}) \\
\text { Mercury, dissolved }(\mu \mathrm{g} / \mathrm{L} \text { as } \mathrm{Hg})\end{array}$ & $\begin{array}{l}13 \\
14 \\
14 \\
12 \\
14\end{array}$ & $\begin{array}{r}53.08 \\
22.07 \\
0.46 \\
0.04 \\
0.42\end{array}$ & $\begin{array}{r}36.14 \\
16.32 \\
0.49 \\
0.12 \\
0.40\end{array}$ & $\begin{array}{l}0.00 \\
9.00 \\
0.00 \\
0.00 \\
0.00\end{array}$ & $\begin{array}{r}120.00 \\
60.00 \\
2.00 \\
0.40 \\
1.60\end{array}$ & $\begin{array}{r}10.02 \\
4.36 \\
0.13 \\
0.03 \\
0.11\end{array}$ \\
\hline $\begin{array}{l}\text { Selenium, total ( } \mu g / L \text { as } \mathrm{Se}) \\
\text { Selenium, suspended total }(\mu \mathrm{g} / \mathrm{L} \text { as } \mathrm{Se}) \\
\text { Selenium, dissolved ( } \mu \mathrm{g} / \mathrm{L} \text { as } \mathrm{Se}) \\
\text { Zinc, total recoverable }(\mu \mathrm{g} / \mathrm{L} \text { as } \mathrm{Zn}) \\
\text { Zinc, suspended recoverable }(\mu \mathrm{g} / \mathrm{L} \text { as } \mathrm{Zn})\end{array}$ & $\begin{array}{l}14 \\
13 \\
14 \\
14 \\
12\end{array}$ & $\begin{array}{r}1.00 \\
0.00 \\
1.00 \\
28.57 \\
22.50\end{array}$ & $\begin{array}{r}0.39 \\
0.00 \\
0.39 \\
16.10 \\
20.94\end{array}$ & $\begin{array}{l}0.00 \\
0.00 \\
0.00 \\
0.00 \\
0.00\end{array}$ & $\begin{array}{r}2.00 \\
0.00 \\
2.00 \\
60.00 \\
60.00\end{array}$ & $\begin{array}{l}0.10 \\
0.00 \\
0.10 \\
4.30 \\
6.05\end{array}$ \\
\hline $\begin{array}{l}\text { Zinc, dissolved }(\mu g / L \text { as } \mathrm{Zn}) \\
\text { Sediment, suspended (mg/L) } \\
\text { Sediment, discharge, suspended (ton/d) }\end{array}$ & $\begin{array}{l}14 \\
37 \\
32\end{array}$ & $\begin{array}{r}7.57 \\
58.51 \\
905.59\end{array}$ & $\begin{array}{r}9.67 \\
91.42 \\
2015.98\end{array}$ & $\begin{array}{l}0.00 \\
3.00 \\
0.57\end{array}$ & $\begin{array}{r}20.00 \\
418.00 \\
8530.00\end{array}$ & $\begin{array}{r}2.58 \\
15.03 \\
356.38\end{array}$ \\
\hline
\end{tabular}


Table 5.--Statistical summary of water-quality data collected at selected surface-water gaging stations in the southern Yampa River basin--Continued

09249750 Williams Fork at mouth, near Hamilton

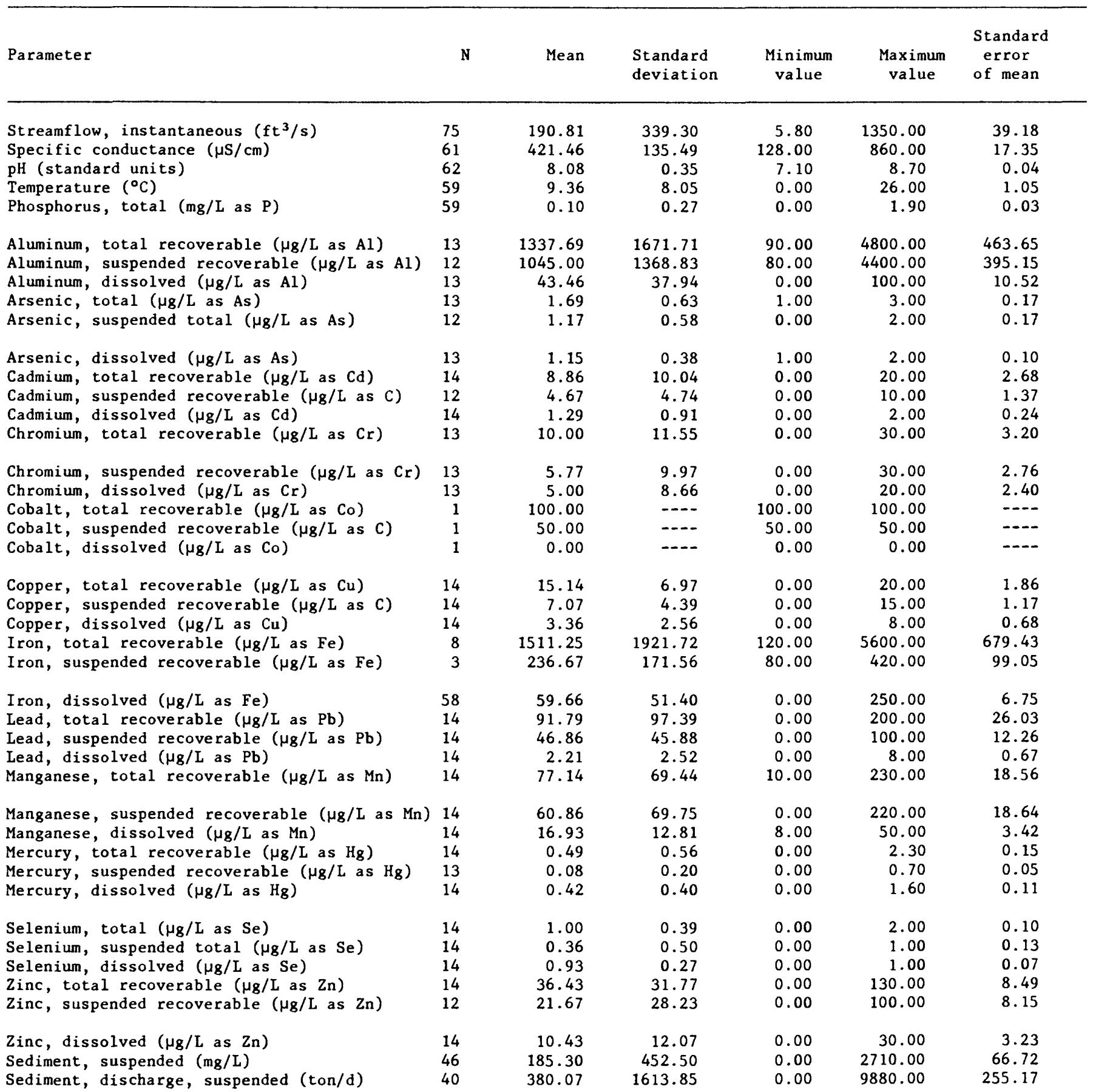




\begin{tabular}{|c|c|c|c|c|c|c|}
\hline Parameter & $\mathrm{N}$ & Mean & $\begin{array}{l}\text { Standard } \\
\text { deviation }\end{array}$ & $\begin{array}{l}\text { Minimum } \\
\text { value }\end{array}$ & $\begin{array}{l}\text { Maximum } \\
\text { value }\end{array}$ & $\begin{array}{l}\text { Standard } \\
\text { error } \\
\text { of mean }\end{array}$ \\
\hline $\begin{array}{l}\text { Streamflow, instantaneous }\left(\mathrm{ft}^{3} / \mathrm{s}\right) \\
\text { Specific conductance }(\mu \mathrm{S} / \mathrm{cm}) \\
\text { pH (standard units) } \\
\left.\text { Temperature ( }{ }^{\circ} \mathrm{C}\right) \\
\text { Phosphorus, total (mg/L as } \mathrm{P})\end{array}$ & $\begin{array}{r}70 \\
42 \\
32 \\
62 \\
3\end{array}$ & $\begin{array}{r}7.29 \\
1567.14 \\
8.17 \\
9.87 \\
0.05\end{array}$ & $\begin{array}{r}47.76 \\
230.81 \\
0.15 \\
7.40 \\
0.05\end{array}$ & $\begin{array}{r}0.02 \\
1100.00 \\
7.90 \\
0.00 \\
0.02\end{array}$ & $\begin{array}{r}400.00 \\
2320.00 \\
8.80 \\
25.00 \\
0.10\end{array}$ & $\begin{array}{r}5.71 \\
35.62 \\
0.03 \\
0.94 \\
0.03\end{array}$ \\
\hline $\begin{array}{l}\text { Aluminum, total recoverable ( } \mu \mathrm{g} / \mathrm{L} \text { as } \mathrm{Al}) \\
\text { Aluminum, suspended recoverable ( } \mu \mathrm{g} / \mathrm{L} \text { as } \mathrm{Al}) \\
\text { Aluminum, dissolved ( } \mu \mathrm{g} / \mathrm{L} \text { as } \mathrm{Al}) \\
\text { Arsenic, total ( } \mu \mathrm{g} / \mathrm{L} \text { as As) } \\
\text { Arsenic, suspended total ( } \mu \mathrm{g} / \mathrm{L} \text { as } \mathrm{As})\end{array}$ & $\begin{array}{l}1 \\
0 \\
0 \\
0 \\
0\end{array}$ & 9500.00 & 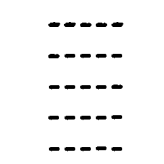 & 9500.00 & 9500.00 & 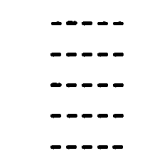 \\
\hline $\begin{array}{l}\text { Arsenic, dissolved ( } \mu g / L \text { as } A s) \\
\text { Cadmium, total recoverable }(\mu g / L \text { as Cd) } \\
\text { Cadmium, suspended recoverable ( } \mu g / L \text { as } C) \\
\text { Cadmium, dissolved }(\mu g / L \text { as } C d) \\
\text { Chromium, total recoverable }(\mu g / L \text { as } C r)\end{array}$ & $\begin{array}{r}9 \\
2 \\
1 \\
10 \\
1\end{array}$ & $\begin{array}{r}1.89 \\
14.50 \\
10.00 \\
1.20 \\
9.00\end{array}$ & $\begin{array}{r}1.69 \\
7.78 \\
-1.03 \\
-1.03\end{array}$ & $\begin{array}{r}0.00 \\
9.00 \\
10.00 \\
0.00 \\
9.00\end{array}$ & $\begin{array}{r}5.00 \\
20.00 \\
10.00 \\
2.00 \\
9.00\end{array}$ & $\begin{array}{r}0.56 \\
5.50 \\
--- \\
0.33 \\
----\end{array}$ \\
\hline $\begin{array}{l}\text { Chromium, suspended recoverable }(\mu \mathrm{g} / \mathrm{L} \text { as } \mathrm{Cr}) \\
\text { Chromium, dissolved }(\mu \mathrm{g} / \mathrm{L} \text { as } \mathrm{Cr}) \\
\text { Cobalt, total recoverable }(\mu \mathrm{g} / \mathrm{L} \text { as Co) } \\
\text { Cobalt, suspended recoverable }(\mu \mathrm{g} / \mathrm{L} \text { as } \mathrm{C}) \\
\text { Cobalt, dissolved }(\mu \mathrm{g} / \mathrm{L} \text { as } \mathrm{Co})\end{array}$ & $\begin{array}{l}0 \\
2 \\
2 \\
1 \\
4\end{array}$ & $\begin{array}{r}10.00 \\
56.00 \\
50.00 \\
0.50\end{array}$ & $\begin{array}{r}14.14 \\
62.23 \\
-1.00\end{array}$ & $\begin{array}{r}----- \\
0.00 \\
12.00 \\
50.00 \\
0.00\end{array}$ & $\begin{array}{r}20.00 \\
100.00 \\
50.00 \\
2.00\end{array}$ & $\begin{array}{r}----- \\
10.00 \\
44.00 \\
---- \\
0.50\end{array}$ \\
\hline $\begin{array}{l}\text { Copper, total recoverable }(\mu \mathrm{g} / \mathrm{L} \text { as } \mathrm{Cu}) \\
\text { Copper, suspended recoverable ( } \mu \mathrm{g} / \mathrm{L} \text { as } \mathrm{C}) \\
\text { Copper, dissolved }(\mu \mathrm{g} / \mathrm{L} \text { as } \mathrm{Cu}) \\
\text { Iron, total recoverable }(\mu \mathrm{g} / \mathrm{L} \text { as } \mathrm{Fe}) \\
\text { Iron, suspended recoverable }(\mu \mathrm{g} / \mathrm{L} \text { as } \mathrm{Fe})\end{array}$ & $\begin{array}{l}2 \\
1 \\
9 \\
2 \\
1\end{array}$ & $\begin{array}{r}13.00 \\
9.00 \\
3.33 \\
2095.00 \\
180.00\end{array}$ & $\begin{array}{r}9.90 \\
-.-- \\
3.84 \\
2694.08 \\
- \\
-\end{array}$ & $\begin{array}{r}6.00 \\
9.00 \\
0.00 \\
190.00 \\
180.00\end{array}$ & $\begin{array}{r}20.00 \\
9.00 \\
13.00 \\
4000.00 \\
180.00\end{array}$ & $\begin{array}{r}7.00 \\
-1.28 \\
1905.00 \\
-.--\end{array}$ \\
\hline $\begin{array}{l}\text { Iron, dissolved }(\mu \mathrm{g} / \mathrm{L} \text { as } \mathrm{Fe}) \\
\text { Lead, total recoverable }(\mu \mathrm{g} / \mathrm{L} \text { as } \mathrm{Pb}) \\
\text { Lead, suspended recoverable ( } \mu \mathrm{g} / \mathrm{L} \text { as } \mathrm{Pb}) \\
\text { Lead, dissolved ( } \mu \mathrm{g} / \mathrm{L} \text { as } \mathrm{Pb}) \\
\left.\text { Manganese, total recoverable ( } \mu_{\mathrm{g}} / \mathrm{L} \text { as } \mathrm{Mn}\right)\end{array}$ & $\begin{array}{r}19 \\
2 \\
1 \\
10 \\
2\end{array}$ & $\begin{array}{r}45.26 \\
110.00 \\
100.00 \\
3.30 \\
160.00\end{array}$ & $\begin{array}{r}42.74 \\
127.28 \\
-.-- \\
3.23 \\
197.99\end{array}$ & $\begin{array}{r}10.00 \\
20.00 \\
100.00 \\
0.00 \\
20.00\end{array}$ & $\begin{array}{r}200.00 \\
200.00 \\
100.00 \\
11.00 \\
300.00\end{array}$ & $\begin{array}{r}9.80 \\
90.00 \\
-1.02 \\
140.00\end{array}$ \\
\hline $\begin{array}{l}\text { Manganese, suspended recoverable }(\mu g / L \text { as } M n) \\
\text { Manganese, dissolved }(\mu g / L \text { as } M n) \\
\text { Mercury, total recoverable }(\mu g / L \text { as } \mathrm{Hg}) \\
\text { Mercury, suspended recoverable }(\mu g / L \text { as } \mathrm{Hg}) \\
\text { Mercury, dissolved }(\mu \mathrm{g} / \mathrm{L} \text { as } \mathrm{Hg})\end{array}$ & $\begin{array}{r}1 \\
20 \\
1 \\
1 \\
10\end{array}$ & $\begin{array}{r}10.00 \\
84.50 \\
0.50 \\
0.00 \\
0.37\end{array}$ & $\begin{array}{l}--.- \\
8.53 \\
-\cdots .21\end{array}$ & $\begin{array}{r}10.00 \\
10.00 \\
0.50 \\
0.00 \\
0.00\end{array}$ & $\begin{array}{r}10.00 \\
180.00 \\
0.50 \\
0.00 \\
0.50\end{array}$ & $\begin{array}{l}---- \\
13.09 \\
---- \\
--. \\
0.07\end{array}$ \\
\hline $\begin{array}{l}\text { Selenium, total ( } \mu g / L \text { as } \mathrm{Se}) \\
\text { Selenium, suspended total ( } \mu \mathrm{g} / \mathrm{L} \text { as Se) } \\
\text { Selenium, dissolved ( } \mu \mathrm{g} / \mathrm{L} \text { as } \mathrm{Se}) \\
\text { Zinc, total recoverable ( } \mu \mathrm{g} / \mathrm{L} \text { as } \mathrm{Zn}) \\
\mathrm{Zinc} \text {, suspended recoverable }(\mu \mathrm{g} / \mathrm{L} \text { as } \mathrm{Zn})\end{array}$ & $\begin{array}{l}1 \\
1 \\
8 \\
2 \\
1\end{array}$ & $\begin{array}{r}2.00 \\
0.00 \\
2.00 \\
24.00 \\
10.00\end{array}$ & 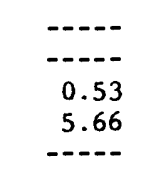 & $\begin{array}{r}2.00 \\
0.00 \\
1.00 \\
20.00 \\
10.00\end{array}$ & $\begin{array}{r}2.00 \\
0.00 \\
3.00 \\
28.00 \\
10.00\end{array}$ & $\begin{array}{r}--- \\
--- \\
0.19 \\
4.00 \\
----\end{array}$ \\
\hline $\begin{array}{l}\text { Zinc, dissolved }(\mu g / L \text { as } Z n) \\
\text { Sediment, suspended }(m g / L) \\
\text { Sediment, discharge, suspended (ton/d) }\end{array}$ & $\begin{array}{l}10 \\
66 \\
66\end{array}$ & $\begin{array}{r}13.00 \\
143.71 \\
7.34\end{array}$ & $\begin{array}{r}9.49 \\
131.38 \\
52.37\end{array}$ & $\begin{array}{r}0.00 \\
12.00 \\
0.00\end{array}$ & $\begin{array}{r}20.00 \\
812.00 \\
426.00\end{array}$ & $\begin{array}{r}3.00 \\
16.17 \\
6.45\end{array}$ \\
\hline
\end{tabular}


Table 5.--Statistical summary of water-quality data collected at selected surface-water gaging stations in the southern Yampa River basin--Continued

09251000 Yampa River near Maybell

\begin{tabular}{|c|c|c|c|c|c|c|}
\hline Parameter & $\mathbf{N}$ & Mean & $\begin{array}{l}\text { Standard } \\
\text { deviation }\end{array}$ & $\begin{array}{l}\text { Minimum } \\
\text { value }\end{array}$ & $\begin{array}{l}\text { Maximum } \\
\text { value }\end{array}$ & $\begin{array}{l}\text { Standard } \\
\text { error } \\
\text { of mean }\end{array}$ \\
\hline $\begin{array}{l}\text { Streamflow, instantaneous }\left(\mathrm{ft}^{3} / \mathrm{s}\right) \\
\text { Specific conductance }(\mu \mathrm{S} / \mathrm{cm}) \\
\text { pH (standard units) } \\
\left.\text { Temperature ( }{ }^{\circ} \mathrm{C}\right) \\
\text { Phosphorus, total (mg/L as P) }\end{array}$ & $\begin{array}{r}200 \\
774 \\
768 \\
418 \\
93\end{array}$ & $\begin{array}{r}1758.22 \\
422.59 \\
7.70 \\
9.43 \\
0.12\end{array}$ & $\begin{array}{r}2911.78 \\
178.54 \\
0.38 \\
7.97 \\
0.20\end{array}$ & $\begin{array}{r}31.80 \\
100.00 \\
6.60 \\
0.00 \\
0.01\end{array}$ & $\begin{array}{r}13,600.00 \\
1,100.00 \\
8.90 \\
28.00 \\
1.60\end{array}$ & $\begin{array}{r}205.89 \\
6.42 \\
0.01 \\
0.39 \\
0.02\end{array}$ \\
\hline 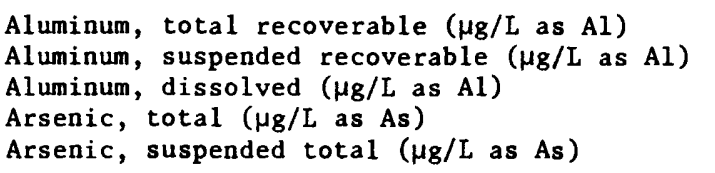 & $\begin{array}{r}2 \\
0 \\
7 \\
30 \\
21\end{array}$ & $\begin{array}{r}2600.00 \\
30.00 \\
1.73 \\
1.43\end{array}$ & $\begin{array}{r}1555.63 \\
14.14 \\
1.64 \\
1.91\end{array}$ & $\begin{array}{r}1500.00 \\
20.00 \\
0.00 \\
0.00\end{array}$ & \begin{tabular}{r}
$3,700.00$ \\
\hdashline 60.00 \\
9.00 \\
9.00
\end{tabular} & $\begin{array}{r}1100.00 \\
5.35 \\
0.30 \\
0.42\end{array}$ \\
\hline $\begin{array}{l}\text { Arsenic, dissolved }\left(\mu_{g} / L \text { as } A s\right) \\
\text { Cadmium, total recoverable }(\mu g / L \text { as Cd) } \\
\text { Cadmium, suspended recoverable }(\mu g / L \text { as } C) \\
\text { Cadmium, dissolved }(\mu g / L \text { as } C d) \\
\text { Chromium, total recoverable }(\mu g / L \text { as } C r)\end{array}$ & $\begin{array}{l}38 \\
30 \\
19 \\
39 \\
29\end{array}$ & $\begin{array}{l}1.16 \\
8.40 \\
5.47 \\
1.31 \\
8.97\end{array}$ & $\begin{array}{l}0.37 \\
9.65 \\
4.51 \\
0.80 \\
8.60\end{array}$ & $\begin{array}{l}1.00 \\
0.00 \\
0.00 \\
0.00 \\
0.00\end{array}$ & $\begin{array}{r}2.00 \\
20.00 \\
10.00 \\
3.00 \\
20.00\end{array}$ & $\begin{array}{l}0.06 \\
1.76 \\
1.04 \\
0.13 \\
1.60\end{array}$ \\
\hline $\begin{array}{l}\text { Chromium, suspended recoverable }(\mu \mathrm{g} / \mathrm{L} \text { as } \mathrm{Cr}) \\
\text { Chromium, dissolved }(\mu \mathrm{g} / \mathrm{L} \text { as } \mathrm{Cr}) \\
\text { Cobalt, total recoverable }\left(\mu_{\mathrm{g}} / \mathrm{L} \text { as Co) }\right. \\
\text { Cobalt, suspended recoverable }\left(\mu_{\mathrm{g}} / \mathrm{L} \text { as } \mathrm{C}\right) \\
\text { Cobalt, dissolved }(\mu \mathrm{g} / \mathrm{L} \text { as } \mathrm{Co})\end{array}$ & $\begin{array}{l}23 \\
38 \\
30 \\
19 \\
39\end{array}$ & $\begin{array}{r}3.48 \\
6.50 \\
41.27 \\
29.37 \\
2.13\end{array}$ & $\begin{array}{r}6.47 \\
8.06 \\
48.84 \\
24.66 \\
3.25\end{array}$ & $\begin{array}{l}0.00 \\
0.00 \\
0.00 \\
0.00 \\
0.00\end{array}$ & $\begin{array}{r}20.00 \\
20.00 \\
100.00 \\
50.00 \\
20.00\end{array}$ & $\begin{array}{l}1.35 \\
1.31 \\
8.92 \\
5.66 \\
0.52\end{array}$ \\
\hline $\begin{array}{l}\text { Copper, total recoverable }(\mu g / L \text { as } \mathrm{Cu}) \\
\text { Copper, suspended recoverable }(\mu \mathrm{g} / \mathrm{L} \text { as } \mathrm{C}) \\
\text { Copper, dissolved }(\mu \mathrm{g} / \mathrm{L} \text { as } \mathrm{Cu}) \\
\text { Iron, total recoverable }(\mu \mathrm{g} / \mathrm{L} \text { as } \mathrm{Fe}) \\
\text { Iron, suspended recoverable }(\mu \mathrm{g} / \mathrm{L} \text { as } \mathrm{Fe})\end{array}$ & $\begin{array}{l}30 \\
29 \\
39 \\
68 \\
16\end{array}$ & $\begin{array}{r}26.43 \\
17.97 \\
5.41 \\
763.24 \\
1298.75\end{array}$ & $\begin{array}{r}64.47 \\
64.49 \\
9.12 \\
1916.21 \\
1947.61\end{array}$ & $\begin{array}{r}2.00 \\
0.00 \\
0.00 \\
20.00 \\
10.00\end{array}$ & $\begin{array}{r}360.00 \\
350.00 \\
58.00 \\
13,000.00 \\
6,200.00\end{array}$ & $\begin{array}{r}11.77 \\
11.97 \\
1.46 \\
232.38 \\
486.90\end{array}$ \\
\hline 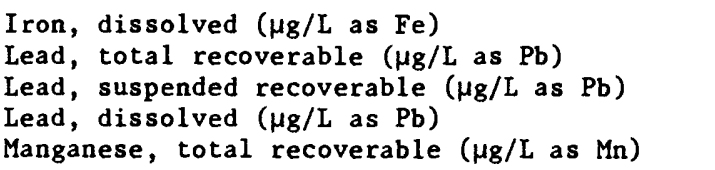 & $\begin{array}{r}177 \\
30 \\
27 \\
39 \\
30\end{array}$ & $\begin{array}{r}73.50 \\
83.67 \\
42.52 \\
2.33 \\
64.67\end{array}$ & $\begin{array}{r}61.92 \\
96.70 \\
47.18 \\
3.74 \\
70.45\end{array}$ & $\begin{array}{r}3.00 \\
0.00 \\
0.00 \\
0.00 \\
20.00\end{array}$ & $\begin{array}{r}370.00 \\
200.00 \\
100.00 \\
23.00 \\
350.00\end{array}$ & $\begin{array}{r}4.65 \\
17.66 \\
9.08 \\
0.60 \\
12.86\end{array}$ \\
\hline $\begin{array}{l}\text { Manganese, suspended recoverable }\left(\mu_{g} / L \text { as Mn) }\right. \\
\left.\text { Manganese, dissolved ( } \mu_{g} / \mathrm{L} \text { as } \mathrm{Mn}\right) \\
\text { Mercury, total recoverable }\left(\mu_{g} / \mathrm{L} \text { as } \mathrm{Hg}_{\mathrm{g}}\right) \\
\text { Mercury, suspended recoverable }\left(\mu_{\mathrm{g}} / \mathrm{L} \text { as } \mathrm{Hg}\right) \\
\text { Mercury, dissolved }\left(\mu_{\mathrm{g}} / \mathrm{L} \text { as } \mathrm{Hg}\right)\end{array}$ & $\begin{array}{l}29 \\
71 \\
29 \\
23 \\
39\end{array}$ & $\begin{array}{r}51.38 \\
14.11 \\
0.49 \\
0.13 \\
0.33\end{array}$ & $\begin{array}{r}63.40 \\
10.62 \\
0.94 \\
0.43 \\
0.64\end{array}$ & $\begin{array}{l}0.00 \\
0.00 \\
0.00 \\
0.00 \\
0.00\end{array}$ & $\begin{array}{r}300.00 \\
50.00 \\
4.90 \\
1.90 \\
4.00\end{array}$ & $\begin{array}{r}11.77 \\
1.26 \\
0.18 \\
0.09 \\
0.10\end{array}$ \\
\hline $\begin{array}{l}\text { Selenium, total ( } \mu g / L \text { as } \mathrm{Se}) \\
\text { Selenium, suspended total }(\mu \mathrm{g} / \mathrm{L} \text { as } \mathrm{Se}) \\
\text { Selenium, dissolved }\left(\mu_{\mathrm{g}} / \mathrm{L} \text { as } \mathrm{Se}\right) \\
\text { Zinc, total recoverable }(\mu \mathrm{g} / \mathrm{L} \text { as } \mathrm{Zn}) \\
\mathrm{Zinc} \text {, suspended recoverable }\left(\mu_{\mathrm{g}} / \mathrm{L} \text { as } \mathrm{Zn}\right)\end{array}$ & $\begin{array}{l}31 \\
25 \\
39 \\
29 \\
24\end{array}$ & $\begin{array}{r}1.19 \\
0.20 \\
1.33 \\
37.24 \\
26.21\end{array}$ & $\begin{array}{r}1.19 \\
0.41 \\
1.18 \\
34.42 \\
29.61\end{array}$ & $\begin{array}{l}0.00 \\
0.00 \\
0.00 \\
0.00 \\
0.00\end{array}$ & $\begin{array}{r}7.00 \\
1.00 \\
7.00 \\
150.00 \\
130.00\end{array}$ & $\begin{array}{l}0.21 \\
0.08 \\
0.19 \\
6.39 \\
6.04\end{array}$ \\
\hline $\begin{array}{l}\text { Zinc, dissolved }(\mu g / L \text { as } Z n) \\
\text { Sediment, suspended (mg/L) } \\
\text { Sediment, discharge, suspended (ton } / d)\end{array}$ & $\begin{array}{r}39 \\
144 \\
129\end{array}$ & $\begin{array}{r}12.74 \\
167.74 \\
2650.25\end{array}$ & $\begin{array}{r}15.33 \\
315.48 \\
6940.21\end{array}$ & $\begin{array}{l}0.00 \\
2.00 \\
0.93\end{array}$ & $\begin{array}{r}70.00 \\
2,080.00 \\
47,200.00\end{array}$ & $\begin{array}{r}2.45 \\
26.29 \\
611.05\end{array}$ \\
\hline
\end{tabular}

